$$
\text { A. } 512^{-1 / 12}
$$

\title{
Distribution of Radionuclides in the Peach Bottom HTGR Primary Circuit During Core 2 Operation
}
F. F. Dyer
R. P. Wichner
W. J. Martın
H. J. de Nordwall

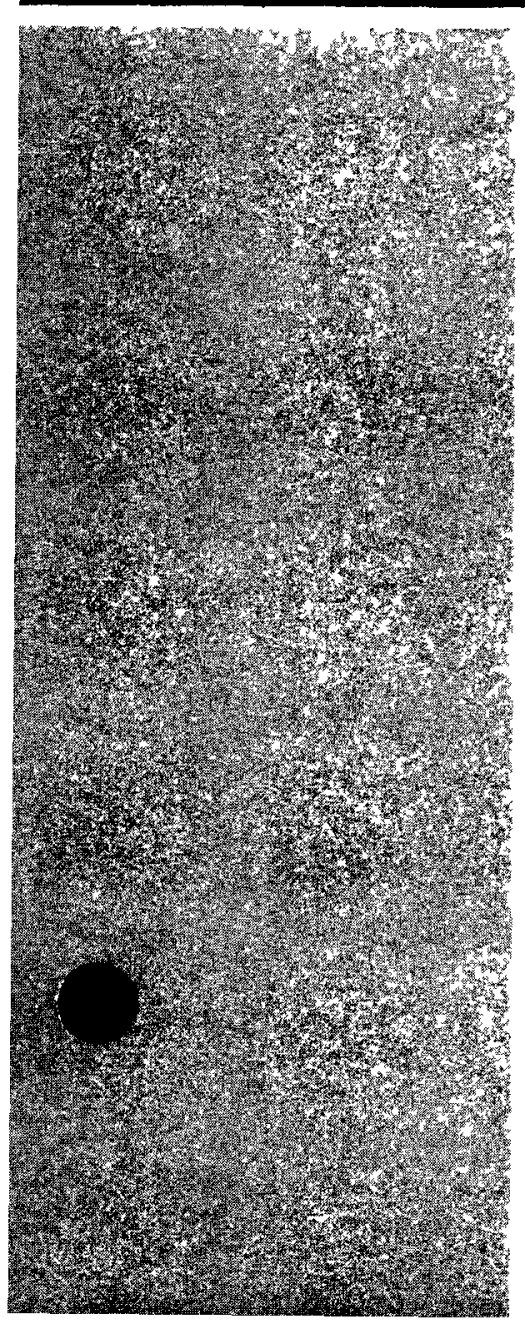

\section{OAK RIDGE NATIONAL LABORATORY}

OPERATED BY UNION CARBIDE CORPORATION FOR THE ENERGY RESEARCH AND DEVELOPMENT ADMINISTRATION 


\section{Printed in the United States of America. Avallable from National Technical Information Service \\ U.S. Department of Commerce \\ 5285 Port Royal Road, Sprıngfield, Virginia 22161 \\ Price: Printed Copy $\$ 7.50$; Microfiche $\$ 3.00$}

This report was prepared as an account of work sponsored by the United States Government Neither the United States nor the Energy Research and Development Admınıstration/United States Nuclear Regulatory Commission, nor any of their employees, nor any of their contractors, subcontractors, or their employees, makes any warranty, express or implied, or assumes any legal liability or responsibility for the accuracy, completeness or usefulness of any information, apparatus, product or process disclosed, or represents that its use would not infringe privately owned rights 


\section{DISCLAIMER}

This report was prepared as an account of work sponsored by an agency of the United States Government. Neither the United States Government nor any agency Thereof, nor any of their employees, makes any warranty, express or implied, or assumes any legal liability or responsibility for the accuracy, completeness, or usefulness of any information, apparatus, product, or process disclosed, or represents that its use would not infringe privately owned rights. Reference herein to any specific commercial product, process, or service by trade name, trademark, manufacturer, or otherwise does not necessarily constitute or imply its endorsement, recommendation, or favoring by the United States Government or any agency thereof. The views and opinions of authors expressed herein do not necessarily state or reflect those of the United States Government or any agency thereof. 


\section{DISCLAIMER}

Portions of this document may be illegible in electronic image products. Images are produced from the best available original document. 
ORNL-5188

Dist. Category UC-77

Contract No. W-7405-eng-26

CHEMICAL TECHNOLOGY DIVISION

HTGR BASE TECHNOLOGY PROGRAM

HTGR Chemistry Studies (189a 10001)

DISTRIBUTION OF RADIONUCLIDES IN THE PEACH BOTTOM HTGR PRTMARY CIRCUIT DURING

CORE 2 OPERATION

F. F. Dyer*

R. P. Wichner

W. J. Martin

H. J. de Nordwall**

\footnotetext{
* On loan from Analytical Chemistry Division **

Present address: Commission Communautes Europeennes, Rue de la Loi 200, 1040 Brussels, Belgium
}

Date Published: March 1977

OAK RIDGE NATIONAL IABORATORY

Oak Ridge, Tennessee 37830

operated by

UNION CARBIDE CORPORATION

for the

ENERGY RESEARCH AND DEVELOPMENT ADMINISTRATION 
○

0 
1. INTRODUCTION AND SUMMARY $\ldots \ldots \ldots \ldots \ldots \ldots \ldots \ldots \ldots \ldots \ldots \ldots \ldots$

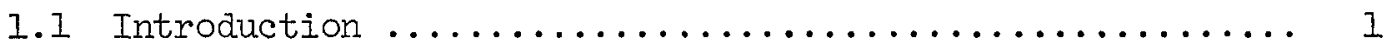

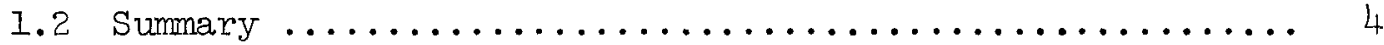

References for section $1 \ldots \ldots \ldots \ldots \ldots \ldots \ldots \ldots \ldots$. 8

2. GAMMA SCANS OF PRIMARY LOOP PIPIING $\ldots \ldots \ldots \ldots \ldots \ldots \ldots \ldots$

2.I Primary Circuit Description and Scanning Locations ..... 8

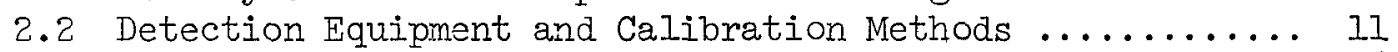

2.3 Data Summary of Primary Loop Gamma scans ............ 16

2.4 Discussion of Primary Loop Gamma Scan Data ........... 23

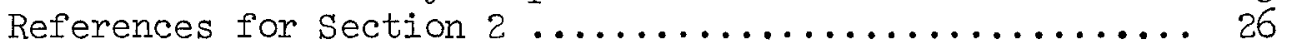

3. PRTMARY COOIANT SAMPLERS FOR GASEOJS RADIONUCLIDES AND

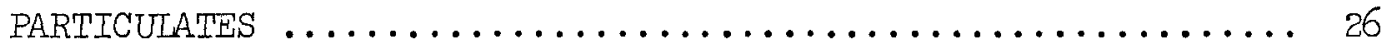

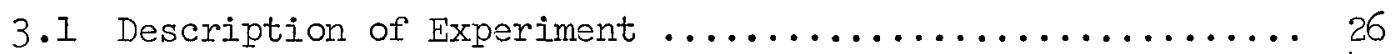

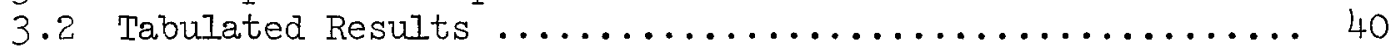

3.2.1 Radionuclide inventories on sample components .... 40

3.2.2 Dust collected on impactor stages and filters .... 40

3.3 Axial Distributions in Sampler Diffusion Tubes

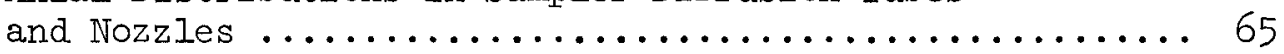

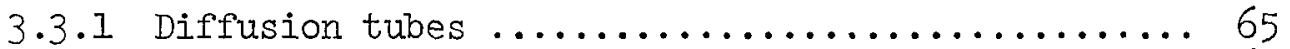

3.3.2 Axial distributions on sampler nozzles ........6 67

3.4 Discussion of Diffusion Thbe Results ............ 77

3.4.1 Theoretical behavior of diffusion tubes ....... 77

3.4.2 Effect of the nozzle on coolant sampler

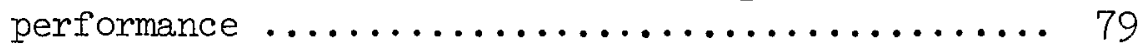

3.4.3 Axial distribution of nuclides on the sampler

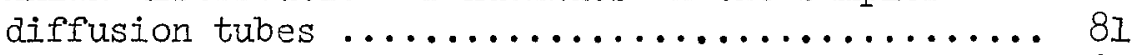
References for section $3 \ldots \ldots \ldots \ldots \ldots \ldots \ldots \ldots$

4. FISSION GAS CONCENTRATIONS IN THE PRIMARY COOIANT AND CORE PURGE GAS .............................. 89

4.1 General Description of Fission Gas Expeximent ......... 89

4.2 Experimental Methods ............................ 91

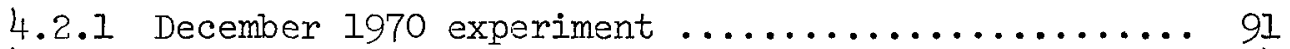

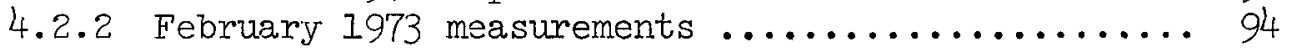

4.2.3 september 1973 measurements ............... 98 
4.3 Release-to-Birth Rate Ratios of Fission Gases ........ 108

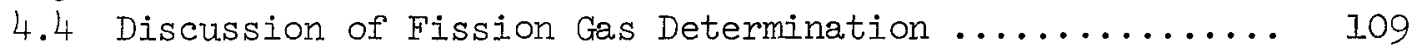

References for section $4 \ldots \ldots \ldots \ldots \ldots \ldots \ldots \ldots \ldots \ldots$

5. ANALYSES OF DUST COLLECTED IN THE CYCLONE SEPARATORS AND

FROM THE EXTERIOR SURFACE OF THE FUEL ELEMENTS ........... 113

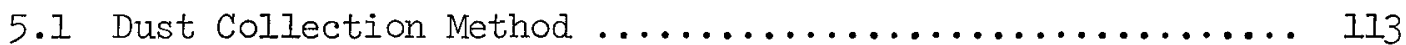

5.2 Summary of Cyclone Separator Dust Data ................ 117

5.3 Density Separation of Sample D3 by Zonal Centrifugation . 124

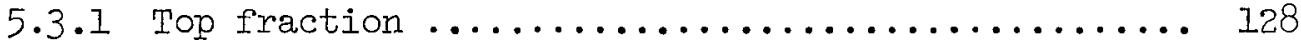

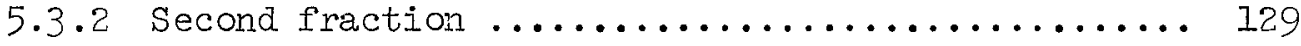

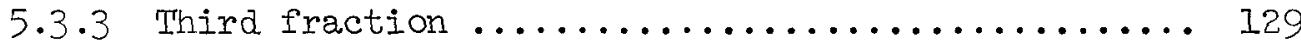

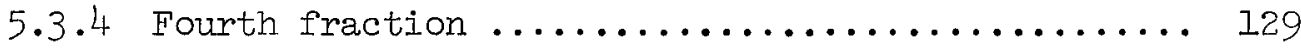

5.4 Scanning Electron Microscope (SEM) Photographs of

Particles in Samples D3 and D4 Size Fractions ......... 130

5.5 Further Particle Size Distribution Data Emphasizing

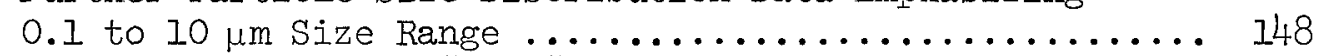

5.6 Characterization of "Soot" Collected from Exterior

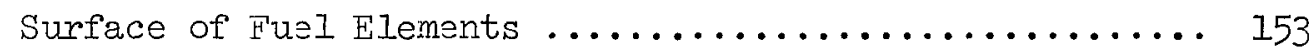

5.7 Dust Concentration and Production Rate in the

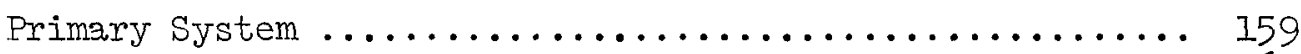

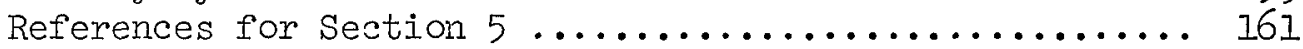

APPENDIX A: DOCUMENTATION OF GAMMA SPECTROSCOPY METHODS $\ldots \ldots \ldots .163$

AI. Reference Nuclear Data Source ................... 163

A2. Measurement of Photopeak Count Rates and Energies ...... 163

A2.I Peak finding and single peak measurement ....... 163

A2.2 Analysis of multiplet peak regions ............ 166

A3. Counting Efficiencies .................... 168

A3.I Usage of counting efficiencies .............. 168

A3.2 Experimental determination of counting

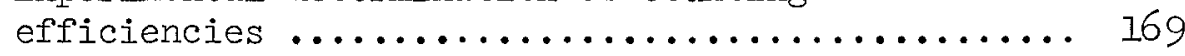

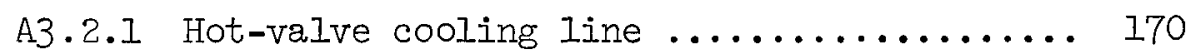

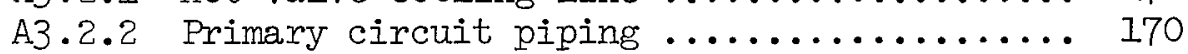

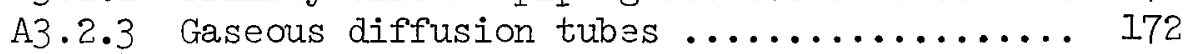

A3.2.4 Purge gas vials and flow cells .......... 172

A4. Formula Used to Compute Activities and Fission

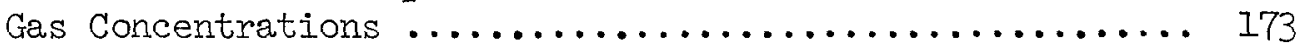

References for Appendix A ........................ 175 
DISTRIBUTION OF RADIONUCIIDES IN THE PEACH BOTTOM HTGR PRIMARY CIRCUIT DURING CORE 2 OPERATION

\author{
F. F. Dyer \\ R. P. Wichner \\ W. J. Martin \\ H. J. de Nordwall*
}

ABSTRACT

A series of measurements regarding the distribution and levels of radioactivity in the primary circuit of the Peach Bottom HTGR was undertaken throughout the course of Core 2 operation, from July 1970, to final shutdown in October 1974. Four types of observations were conducted in this phase of the Peach Bottom Surveillance Program: (I) the cold duct of the primary circuit was axially scanned for gamma-emitting nuclides on four occasions, including the last which directly followed the final shutdown, (2) the primary coolant was sampled upstream and downstream from the steam generator through diffusion tubes and filters to differentiate between gaseous- and particulate-borne activity, (3) the primary coolant and fuel-element purge flow were rapidly sampled for fission gas measurement, and (4) dust samples collected using the cyclone separators in the primary loop were examined for size distribution, chemical makeup, and contained activity.

The various measurements undertaken are described, and the results are presented in tabular and graphical form. Sufficient documentations of experimental procedures are provided to allow continued analyses of primary circuit fission-product distributions to be performed.

\title{
1. INTRODUCTION AND SUMMARY
}

\section{I Introduction}

This report describes the experiments conducted and summarizes the data acquired in the course of the surveillance activities of the Peach Bottom HTGR primary circuit during Core 2 operation. As such, it presents

\footnotetext{
* Present address: Commission Communautes Europeennes, Rue de la Loi 200, 1040 Brussels, Belgium.
} 
the results of a portion of the Peach Bottom Surveillance Program, an ongoing program at ORNL since 1970, the activities of which could, in general, be separated into two broad categories: (1) driver-fuel-element examinations $^{1,2}$ and (2) examinations of fission-product distributions in the primary circuit.

As a part of the primary circuit investigation, gamma-spectra were acquired at approximately eight locations along the primary loop duct work on four occasions during scheduled maintenance shutdowns. This effort, conducted to measure plateout activities in the primary circuit, is described, and the data is summarized in sect. 2. Section 3 investigates gasborne activity by means of coolant samplers operating isokinetically upstream and downstream from the steam generator. These gas samples were drawn through diffusion tubes in order to distinguish between molecular and particulate activity and were finally drawn through various types of filters to ensure the capture of all the sample activity. The coolant sampling experiments described in sect. 4 were designed for a much shorter delay time between the extraction and the analysis and were intended specifically for the purpose of detecting the shorter-duration krypton and xenon nuclides resulting from fission. These fission gas experiments were performed on three occasions, and a number of different methods for capturing the gas sample was employed. Finally, Sect. 5 deals with the examination of the dust collected in the cyclone separator located in the primary circuit. Specific activity, chemical makeup, and particle-size distribution data are given.

Although each section contains a full description of the experiment, including the dates involved, the reader may find it helpful to occasionally refer to Table 1.1, which lists the operating history of Core 2 . For reference, the first primary circuit gamma scan was performed shortly after the first shutdown on Apr. 17, 1971. It was also during this shutdown that the first ORNL coolant sampling device was installed and the first dust sample was acquired. The final gamma-scan and dust samples were acquired shortly following the termination of Core 2 operation on oct. 31, 1974. 
Table 1.1. Peach Bottom HTGR operating history and ORNL surveillance activities

\begin{tabular}{|c|c|c|c|c|c|c|c|}
\hline Date & $\begin{array}{l}\text { Time } \\
\text { point }\end{array}$ & 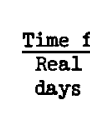 & 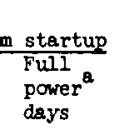 & $\begin{array}{c}\text { Durati } \\
\text { time } \\
\text { Real } \\
\text { days }\end{array}$ & $\begin{array}{l}\begin{array}{l}\text { n between } \\
\text { points }\end{array} \\
\begin{array}{l}\text { Full } \\
\text { power } \\
\text { days }\end{array}\end{array}$ & $\begin{array}{l}\text { Av. power } \\
\text { during } \\
\text { period } \\
{[\mathrm{MW}(t)]}\end{array}$ & Survelllance activity \\
\hline \multirow[t]{5}{*}{ July 15, 1970} & 1 & 0 & 0 & & & & \\
\hline & 2 & 28.8 & 27.0 & 28.8 & 27.0 & 107.6 & \\
\hline & 3 & 67.7 & 62.7 & 38.8 & 35.7 & 107.6 & \\
\hline & 4 & 75.2 & 68.6 & 7.5 & 5.9 & 89.9 & \\
\hline & 5 & 216.1 & 202 & 140.9 & 133.5 & 108.9 & First fission gas run. \\
\hline \multirow{6}{*}{$\begin{array}{l}\text { Apr. 22, } 1971 \\
\text { July 4, } 1971\end{array}$} & 6 & 276.1 & 252.4 & 60.0 & 50.3 & 96.5 & \\
\hline & 7 & 356.1 & 252.4 & 80.0 & 0 & 0 & $\begin{array}{l}\text { First primary circuft ganma scan; } \\
\text { dust sample D2. }\end{array}$ \\
\hline & 8 & 412.5 & 298.0 & 56.4 & 45.6 & 93.0 & $\begin{array}{l}\text { Cl deffusion tube sampler begins } \\
\text { operation. }\end{array}$ \\
\hline & 9 & 468.1 & 343.0 & 55.6 & 45.0 & 93.0 & \\
\hline & 10 & 490.1 & 343.0 & 22.0 & 0 & 0 & \\
\hline & 71 & 537.1 & 385,4 & 47.0 & 42.4 & 104.0 & \\
\hline \multirow{7}{*}{ Jan. 7, 1972} & 12 & 699.1 & 385.4 & 162.0 & 0 & 0 & $\begin{array}{l}\text { Second gamma scan; removal of first } \\
\text { driver fuel element for PIE; CA-1 } \\
\text { dfffuslon-tube sampler installed. }\end{array}$ \\
\hline & 13 & 8450 & 5000 & 145.9 & 114.6 & 90.0 & CA-1 diffusion-tube sampler run. \\
\hline & 14 & 891.0 & 500.0 & 46.0 & 0 & 0 & 0il leak to primary system corrected \\
\hline & & & & 80.6 & 64.1 & 92.0 & \\
\hline & 25 & 971.6 & 564.1 & 51.5 & 46.2 & 103.0 & Second fission gas run. \\
\hline & 16 & 1023.1 & 610.3 & 20.0 & 0 & 0 & \\
\hline & 17 & 1043.1 & 610.3 & 98.8 & 90.9 & 106.0 & Third fission gas run. \\
\hline \multirow[t]{9}{*}{ Sept. 14, 1973} & 18 & 1141.9 & 701.2 & 114.0 & 0 & 0 & $\begin{array}{l}\text { Third gama scan; second driver } \\
\text { fuel element acquired; dust sample } \\
\text { D3. }\end{array}$ \\
\hline & 19 & 1255.9 & 701.2 & 50.7 & 46.8 & 106.0 & \\
\hline & 20 & 1306.6 & 748.0 & 50.0 & 40.0 & 92.0 & $\begin{array}{l}\mathrm{CC} \text { and } \mathrm{CD} \text { diffusion tube samplers } \\
\text { begin operation. }\end{array}$ \\
\hline & 21 & 1356.6 & 788.0 & 37.5 & 30.0 & 92.0 & \\
\hline & 22 & 1394.1 & 818.0 & 38.0 & 0 & 0 & \\
\hline & 23 & 1432.1 & 818.0 & 30.0 & 17.0 & 65.5 & \\
\hline & 24 & 1462.1 & 835.0 & 29.0 & 23.0 & 91.5 & \\
\hline & 25 & 1491.1 & 858.0 & 47.0 & 31.7 & $\pi .5$ & \\
\hline & ж & 1538.1 & 889.7 & 12.0 & 7.6 & 68.8 & \\
\hline oct. 31, 1974 & 27 & 1550.1 & 897.3 & & & & Fourth gamm scan; D4 dust sample. \\
\hline
\end{tabular}

Based on a rated rull power of $115 \mathrm{w}(\mathrm{t})$. 
Naturally, surveillance experiments of this type on an operating reactor require the assistance of far more people than appear as authors of this report. I. L. Fairchild of the Chemical Technology Division, ORNL, ably assisted in the setup and performance of the experiments, as well as the procesuing of the data. J. O. Kolb of the ORNL Reactor Division was instrumental in the design of the coolant samplers and, with the assistance of Philadelphia Electric Company personnel, was responsible for the installation and operation of the samplers. R. A. Lorenz of the ORNL Chemical Technology Division designed the cascade impactor used in two of the coolant samplers. Norman E. Baldwin of General Atomic Corporation (GAC) was responsible for hooking up the fission gas-measurement apparatus to the existing gas-sampling system. We would also like to acknowledge the full support and cooperation supplied by the Philadelphia Electric Company and especially W. C. Birely, technical engineer in charge of reactor unit 1 throughout most of duration of the surveillance program, without whose assistance these experiments could not have been performed. Finally, we acknowledge several in the ORNL Analytical Chemistry Division - L. D. Hull.ett for dust characterization by electron and x-ray methods; W. R. Musick for the chemical analysis of dust by emission spectroscopy; L. C. Bate, H. R. Parker, and M. E. Pruitt for measurements of beta-emitting radionuclides and some gamma emitters; and J. F. Emery for ${ }^{235} \mathrm{U}$ determination by delayed neutron counting.

\subsection{Summary}

Primary circuit gamma scans were obtained on four occasions following 252, 385, 701, and 897 equivalent full-power days (EFPDs) of Core 2 operation. The last scan was acquired soon after the final reactor shutdown; the first three scans were taken during scheduled maintenance intervals. The major, observed surface activity was due to the nuclides ${ }^{134} \mathrm{Cs}$ and $137 \mathrm{Cs}$. Initially, $2.2 \mathrm{Ci}$ of ${ }^{137} \mathrm{Cs}$ was found to be deposited on the cold ducts, representing $\sim 0.002 \%$ total core inventory.

The observed surface activity due to ${ }^{137} \mathrm{Cs}$ fell to approximately half the initial value between the first and the second primary circuit scans. Subsequently, the surface activity rose slightly between the second and 
third scans and then rose sharply between the third and fourth scans to a final value of $\sim 8 \mathrm{Ci}$ of ${ }^{137} \mathrm{Cs}$. The initial high value may be attributed to the residue left from Core 1 operation, where substantially higher fuel-failure fractions were experienced, and the sharp rise observed in the final year of operation is attributed to the diffusive breakthrough of $\mathrm{Cs}$ generated during Core 2 operation. This view is supported by the observed decline in the isotopic ratio, ${ }^{137} \mathrm{Cs} /{ }^{134} \mathrm{Cs}$, during this period.

other gamma activity generally observed on the primary circuit cold ducts were the nuclides: ${ }^{54} \mathrm{Mn},{ }^{59} \mathrm{Fe},{ }^{60} \mathrm{Co},{ }^{65} \mathrm{Zn}, 11 \mathrm{~mm}_{\mathrm{Ag}},{ }^{140} \mathrm{Ba}$, and ${ }^{144} \mathrm{Ce}$. On the occasion of the third gamma scan, when conditions were most advantageous, the distribution of ${ }^{13} I_{I}$ along the cold duct was measured; at other times, only upper limit values of iodine plateout activity were given. Other nuclides occasionally positively identified on the cold duct were: ${ }^{103} \mathrm{Ru},{ }^{106} \mathrm{Ru},{ }^{154} \mathrm{Eu}$, and ${ }^{23} \mathrm{~Pa}$.

A series of six coolant sampling experiments was performed in which the last four were paired and run simultaneously at locations upstream and downstream from the steam generator. Each of these experiments was conducted over a range of 1700 to $6500 \mathrm{hr}$ of reactor-operating time, during which time the coolant was continuously drawn isokinetically through a set of diffusion tubes, filters, and iodine absorbers in order to determine the nature of the transported specie. The amount of dust collected during each of the coolant sampler experiments per unit mass of coolant throughput is summarized below:

\begin{tabular}{cclc}
\hline $\begin{array}{c}\text { Sampler } \\
\text { designation }\end{array}$ & $\begin{array}{c}\text { Operating } \\
\text { dates }\end{array}$ & $\begin{array}{c}\text { Location relative } \\
\text { to steam generator }\end{array}$ & $\begin{array}{c}\text { Dust collected per } \\
\text { unit mass of coolant } \\
\text { throughput } \\
(\mathrm{mg} / \mathrm{kg} \text { coolant })\end{array}$ \\
\hline CG & & upstream & 0.16 \\
$\mathrm{Cl}$ & $7 / 71-12 / 71$ & downstream & 0.041 \\
$\mathrm{CA}-1$ & $9 / 72-11 / 72$ & upstream & 0.039 \\
$\mathrm{CA}-2$ & $1 / 73-5 / 73$ & upstream & 0.070 \\
$\mathrm{CB}$ & $1 / 73-5 / 73$ & downstream & 0.087 \\
$\mathrm{CC}$ & $3 / 74-5 / 74$ & upstream & 0.029 \\
$\mathrm{CD}$ & $3 / 74-5 / 74$ & downstream & $<0.002$ \\
\hline
\end{tabular}


Two of the coolant samplers were equipped with impactor plates for classifying the particulates according to size. The analysis of the dust collected on these plates revealed it to be composed primarily of graphite, $\mathrm{Fe}_{3} \mathrm{C}$, and an unknown iron-silicon compound which left no diffraction pattern.

All of the coolant samplers were equipped with diffusion tubes for distinguishing between molecular and particulate-borne activity. In theory, an observed, steep, axial slope along the tube signifies molecular transport, whereas a flat profile results from the low values of diffusion coefficients that are effective for particulates. A number of Cs profiles (but not all) do indeed conform to this theoretical model; however, a majority of the observed profiles for ${ }^{110 \mathrm{~m}} \mathrm{Ag},{ }^{131} \mathrm{I}$, and ${ }^{54} \mathrm{Mn}$ appears to be more complex than can be explained by the available, simple deposition model. Hence, full interpretation of the diffusion tube data awaits a more sophisticated analysis than is undertaken here.

Levels of fission gases (isotopes of krypton and xenon) in the primary coolant were determined on three occasions during Core 2 operation-Dec. 1970, Feb. 1973, and Sept. 1973. Fission gas levels in the fuelelement purge gas were also determined in the last series of experiments, and the following release-to-birth rate ratios were obtained:

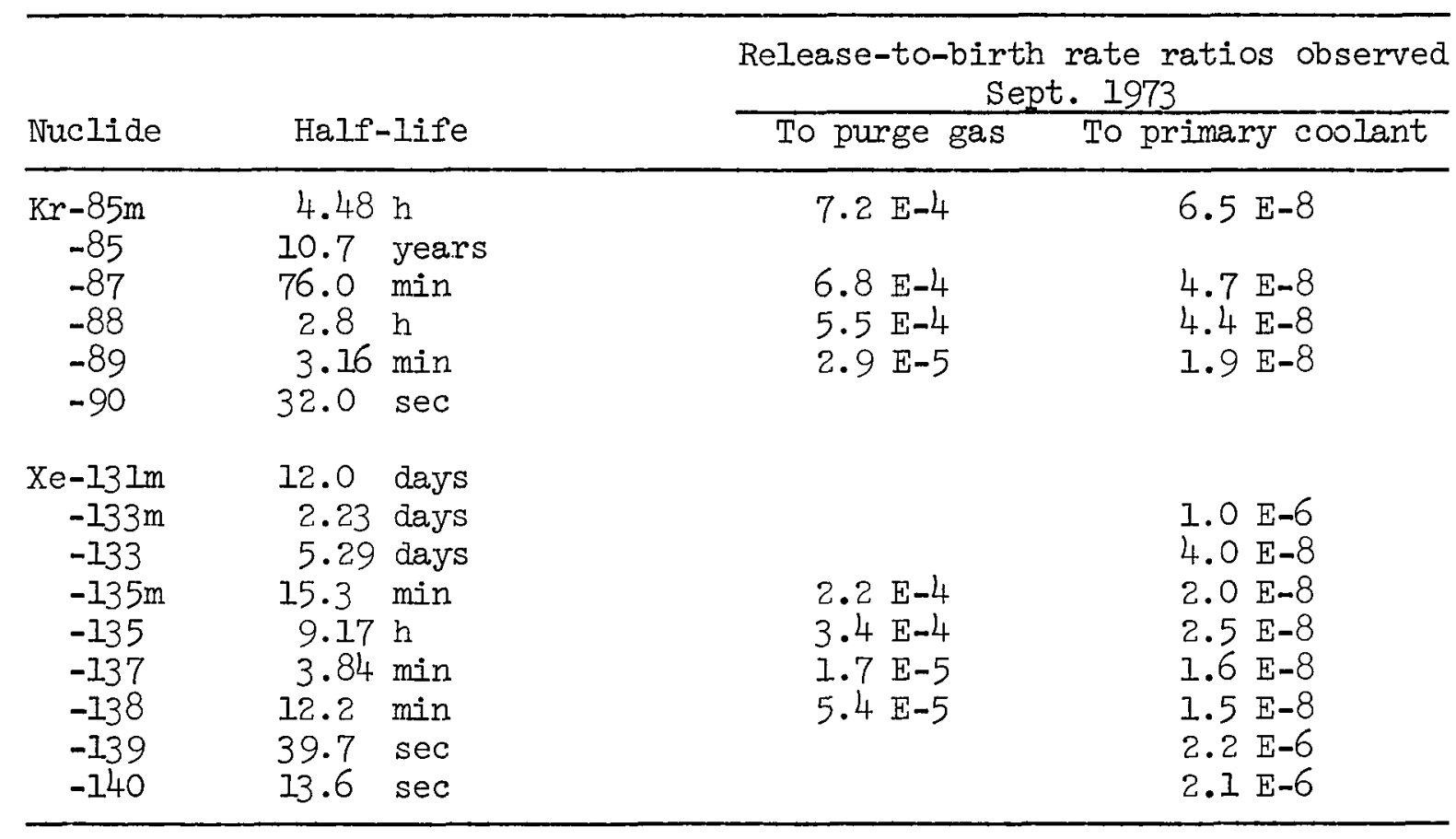


Four different methods for obtaining fission gas levels in the primary coolant and purge gas were employed. These are described in detail, and the relative advantages and disadvantages of each method are discussed. Short-lived nuclides, such as ${ }^{90} \mathrm{Kr},{ }^{139} \mathrm{Xe}$, and ${ }^{140} \mathrm{Xe}$, are best detected using a high pressure flow-through system if clean sample chambers in some primary circuit bypass line have been installed. Long-lived nuclides are best measured by collection on charcoal that is cooled to the liquidnitrogen temperature. Methods that employed the radiochemical sampling facility at Peach Bottom met with some difficulty due to the uncertainty in the value of the transit time and the complexity of the sampling system.

The dust samples collected by the loop-l cyclone separator were acquired on three occasions. The observed sample masses and estimated dust concentrations and dust production rates are presented below. Since the collection efficiency of the cyclone separator departs significantly from unity for particle sizes below the range of $\sim 0.1$ to $0.3 \mu \mathrm{m}$, these data apply to the particulate levels in the primary circuit above this size range.

\begin{tabular}{lccc}
\hline & $\begin{array}{c}\text { Dust concentra- } \\
\text { tion in primary } \\
\text { loop during } \\
\text { Somple mass, } \\
\text { loop l } \\
(\mathrm{g})\end{array}$ & $\begin{array}{l}\text { Rate of dust } \\
\text { production dur- } \\
\text { ing operation }\end{array}$ \\
\hline July 1970-Apr. 1971 & 80.0 & 0.12 & $\begin{array}{l}\mathrm{kg} \text { coolant }) \\
\text { period } \\
(\mathrm{g} / \mathrm{sec})\end{array}$ \\
June 1971-Sept. 1973 & 110.0 & 0.084 & $8.2 \times 10^{-5}$ \\
Jan. 1973-Oct. 1974 & 15.3 & 0.024 & $5.8 \times 10^{-5}$ \\
\hline
\end{tabular}

Scanning electron micrographs of the dust collected beneath the cyclone separators show that the most frequently observed particle is an iron-rich particle (metallic iron--both the oxide and carbide were detected) in the size range of 10 to $40 \mu \mathrm{m}$. Frequently, these particles appear as tightly held agglomerates, achieving sizes up to a few millimeters. A second major constituent of the dust appears to be carbon in both the amorphous and graphite form. On two occasions, pieces of metallic foil 
up to $1.3 \mathrm{~cm}$ in length, evidently from the hot-duct insulation, were found in the dust collection bottle.

Approximately $200 \mathrm{mg}$ of "soot" was collected from each of two fuel elements removed from the core. This black, fine, powdery material appeared as a deposit in the exterior region above the fueled zone and is believed to be one of the sources of circulating particulates in the primary system. Scanning electron micrographs revealed the "soot" to be composed of filaments 0.1 to $0.5 \mu \mathrm{m}$ in width and flake-type particles up to $4 \mu \mathrm{m}$ in size. The major constituents of this material are amorphous carbon, graphite, and iron.

References for Section 1

1. F. F. Dyer et aI., Post-Irradiation Examination of Peach Bottom HTGR Element E05-01, ORNL-5126 (Apr. 1976).

2. R. P. Wichner et al., Distribution of Fission Products in Peach Bottom HTGR Fuel Element Ell-07, ORNL-5214 (to be published).

\section{GAMMA SCANS OF PRTMARY LOOP PIPING}

\subsection{Primary Circuit Description and Scanning Locations}

A schematic of the primary circuit piping is shown in Fig. 2.1-1 which displays the 11 locations where gamma scans were taken during shutdown intervals in Core 2 operation. The primary circuit consists of two loops, each of which contains a helium compressor and a steam generator. The total He flow of $210,000 \mathrm{~kg} / \mathrm{hr}(463,0001 \mathrm{~b} / \mathrm{hr})$ is divided equally between the two loops containing a combined volume of $232 \mathrm{~m}^{3}$ (8200 $\left.\mathrm{ft}^{3}\right)$. All the large duct, pressure-containing material of both primary loops consists of carbon steel. Details regarding valves, compressors, reactor internal flow configuration, and steam-generator tubing geometry are provided in Sect. II-D of the Peach Bottom Final Hazards Summary Report, ${ }^{1}$ and only a few critical features are noted here.

The coolant exits from the reactor in the inner pipe of a concentric duct arrangement consisting of an outer 42-in.-OD pipe with a 3/4-in. wall 


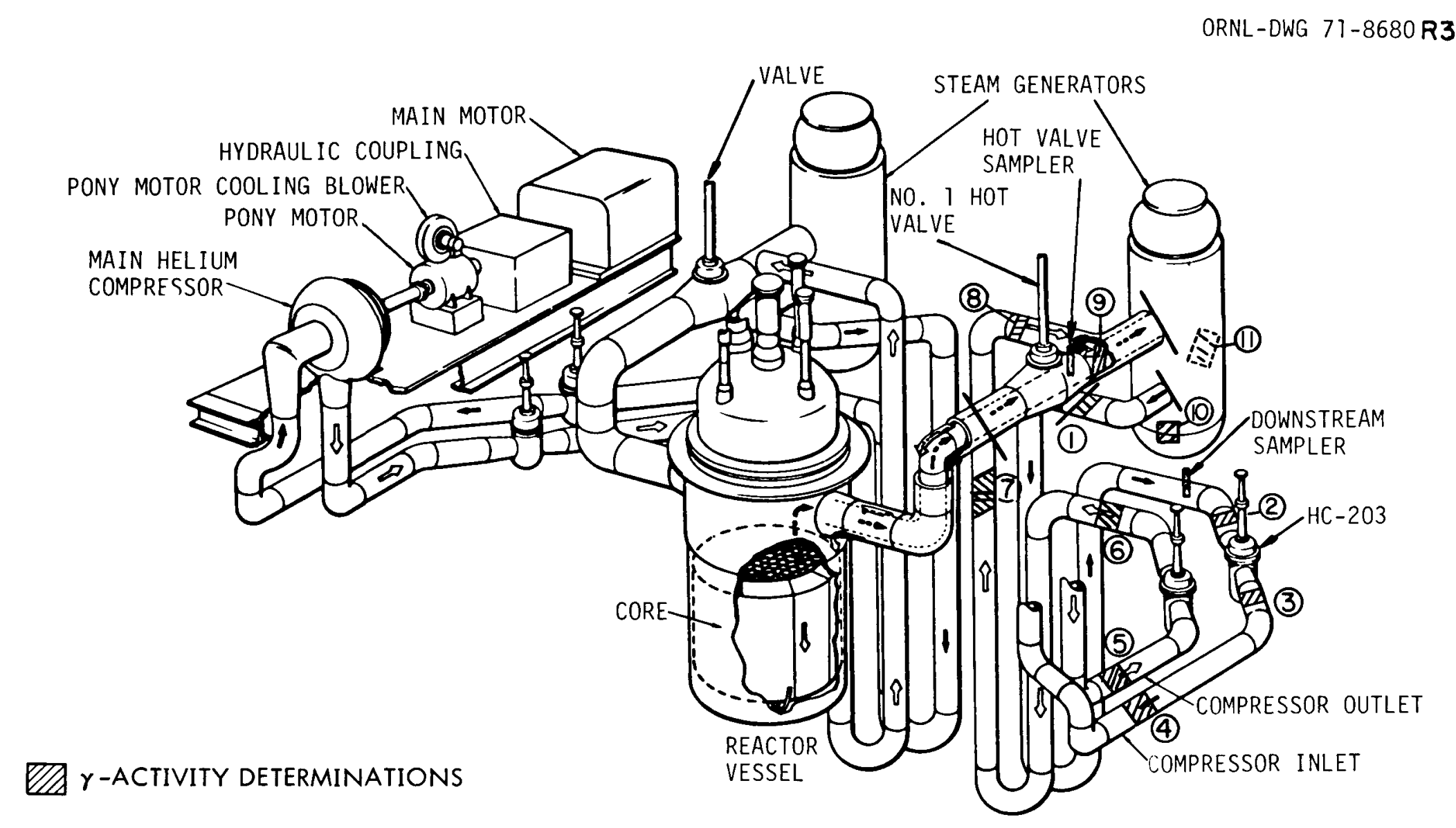

Fig. 2.1-1. Primary loop schematic showing locations for gamma scans and coolant sampler stations. 
thickness and an inner pipe of 30-in.-OD with a 3/8-in. wall thickness. There is a thermal barrier inside the inner pipe that is fabricated from laminated 304 stainless steel and described in Fig. II-88 of ref. 1, which maintains the inner wall temperature below $371{ }^{\circ} \mathrm{C}\left(700^{\circ} \mathrm{F}\right)$. Some gamma spectra of the concentric duct were acquired during a number of the shutdown periods. Since there are present in the duct at this location several distinctly different surfaces that can influence the deposition of activities and the attenuation of gamma rays, an unambiguous interpretation of the spectral data obtained at the concentric duct is difficult.

The cold duct, which extends from the steam generator outlet to the compressor and on to the entry region of the concentric pipe, consists of 28-in.-OD carbon steel pipe (Type A-212, Grade B) with a 7/16-in. wall thickness. Locations where gamma scans were acquired and the approximate distances from the steam generator outlet are identified in Table 2.1-1, where the specified location number relates to sites noted in Fig. 2.I-I and to subsequent tables listing the activity levels.

Table 2.1-1. Primary loop gamma-scan locations; loop I

\begin{tabular}{clc}
\hline $\begin{array}{c}\text { Location } \\
\text { No. }\end{array}$ & \multicolumn{1}{c}{ Location } & $\begin{array}{c}\text { Approximate dis- } \\
\text { tances from loop 1 } \\
\text { generator } \\
(\mathrm{m})\end{array}$ \\
\hline 1 & Near steam generator & 2 \\
2 & Upstream from valve HC 203 & 34 \\
3 & Downstream from valve HC 203 & 37 \\
4 & Compressor inlet, near crawl space & 41 \\
$5^{a}$ & Compressor outlet, near crawl space & 58 \\
$6^{a}$ & Compressor outlet, near steam generator & 67 \\
$7^{a}$ & Vertical duct & 99 \\
$8^{\text {a }}$ & Directly upstream from concentric duct & \\
9 & Concentric duct & \\
10 & Steam generator, below exit duct & \\
11 & Steam generator, back side from exit duct & \\
\hline
\end{tabular}

${ }^{a}$ Includes an equivalent of $12.2 \mathrm{~m}$ for compressor. 


\subsection{Detection Equipment and Calibration Methods}

Gamma spectra of primary circuit ducts were acquired with a $\mathrm{Ge}(\mathrm{Li})$ detector and a Nuclear Data Model 4410 computer-based pulse height analyzer. The analyzer system shown in Fig. 2.2-1 consisted of a Nuclear Data Model 812 computer with a $16 \mathrm{~K}$, 12-bit memory. Peripherals on the system included a teletype, an oscilloscope, a magnetic-tape cassette deck, a fast papertape punch and reader, and an analog-to-digital converter as an input channel for the $\mathrm{Ge}(\mathrm{Li})$ detector. The use of this equipment for acquiring and analyzing gamma-ray spectra has been described previously. ${ }^{2}$

Measurements through 1973 were made with a Nuclear Diodes Ge(Li) detector, which had a resolution of $2.3 \mathrm{keV}$ and a counting efficiency of $4.6 \%$ of a 7.6-cm-diam x $7.6 \mathrm{~cm}$ length cylindrical NaI detector. ${ }^{*}$ This detector was replaced before the 1974 measurements by an Ortec detector with a resolution of $2.1 \mathrm{keV}$ and an efficiency of $\%$.

Localized areas on the duct were measured by the use of a collimator shield surrounding the detector. The detector and necessary shielding were secured in an aluminum frame box during use. The box served to protect the detector and provide a convenient means of accurately relocating the detector within the shield and moving the system between counting losations on the duct. Eye bolts in the top of the box allowed the detector system to be hoisted to counting locations on overhead ducts. The box with the shielding and the Nuclear Diode detector in place is shown in Fig. 2.2-2.

Shielding for the detector consisted of two parts: an annular lead collimator cylinder that surrounded the detector and a rectangular lead shield that surrounded the cryostat from the cylindrical shield back to the Dewar. The cylindrical shield contained collimator openings to provide a path for gamma rays to the detector. The rectangular shield prevented much of the gamma radiation scattered from the surroundings from reaching the detector. This reduction of the scattered radiation, which

\footnotetext{
* These specifications pertain to the photopeak of the $1332-\mathrm{keV}$ gamma ray of ${ }^{60} \mathrm{Co}$.
} 


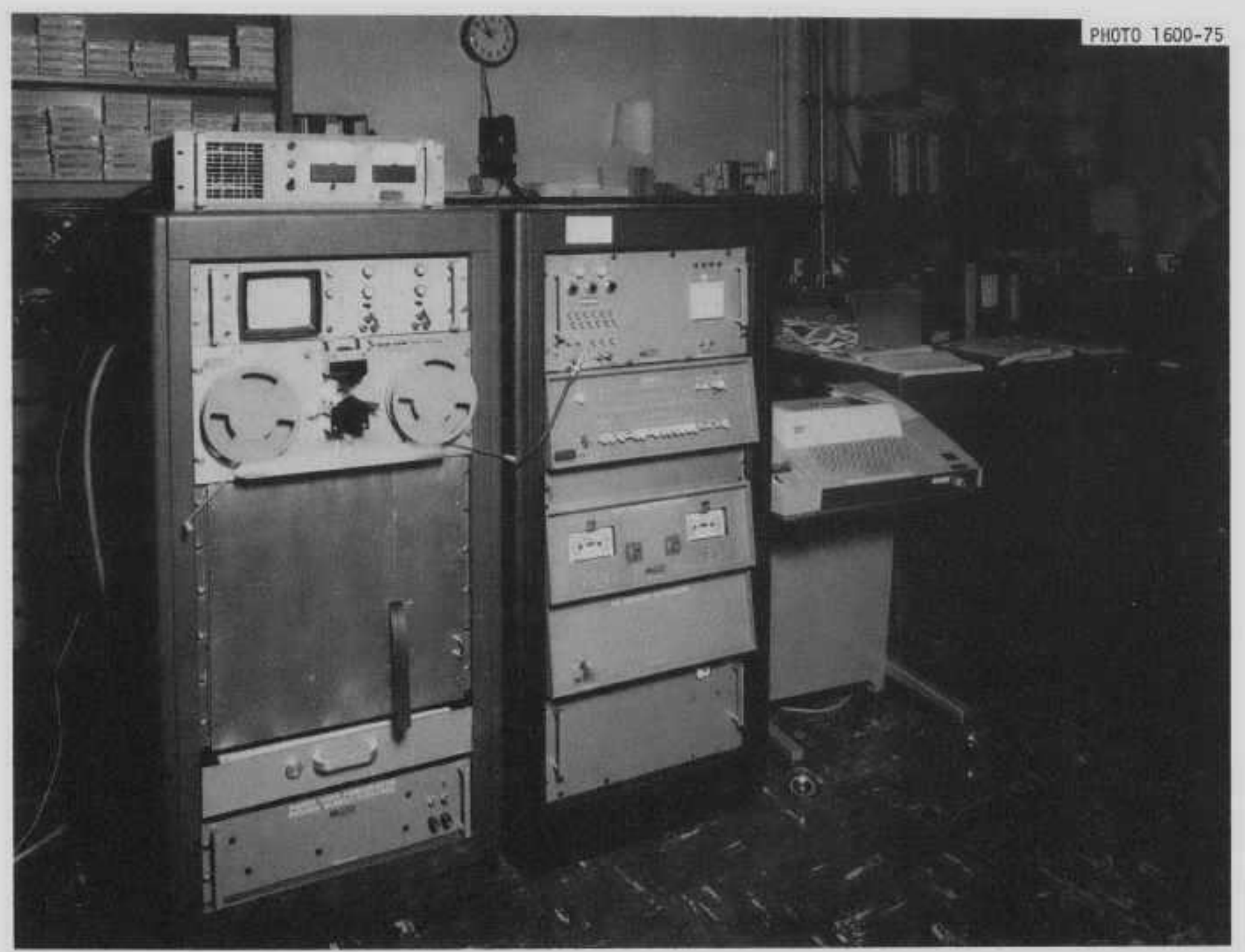

Fig. 2.2-1. Computer based pulse-height analyzer used to acquire Bamma spectra of Finch Bottom ducts. 


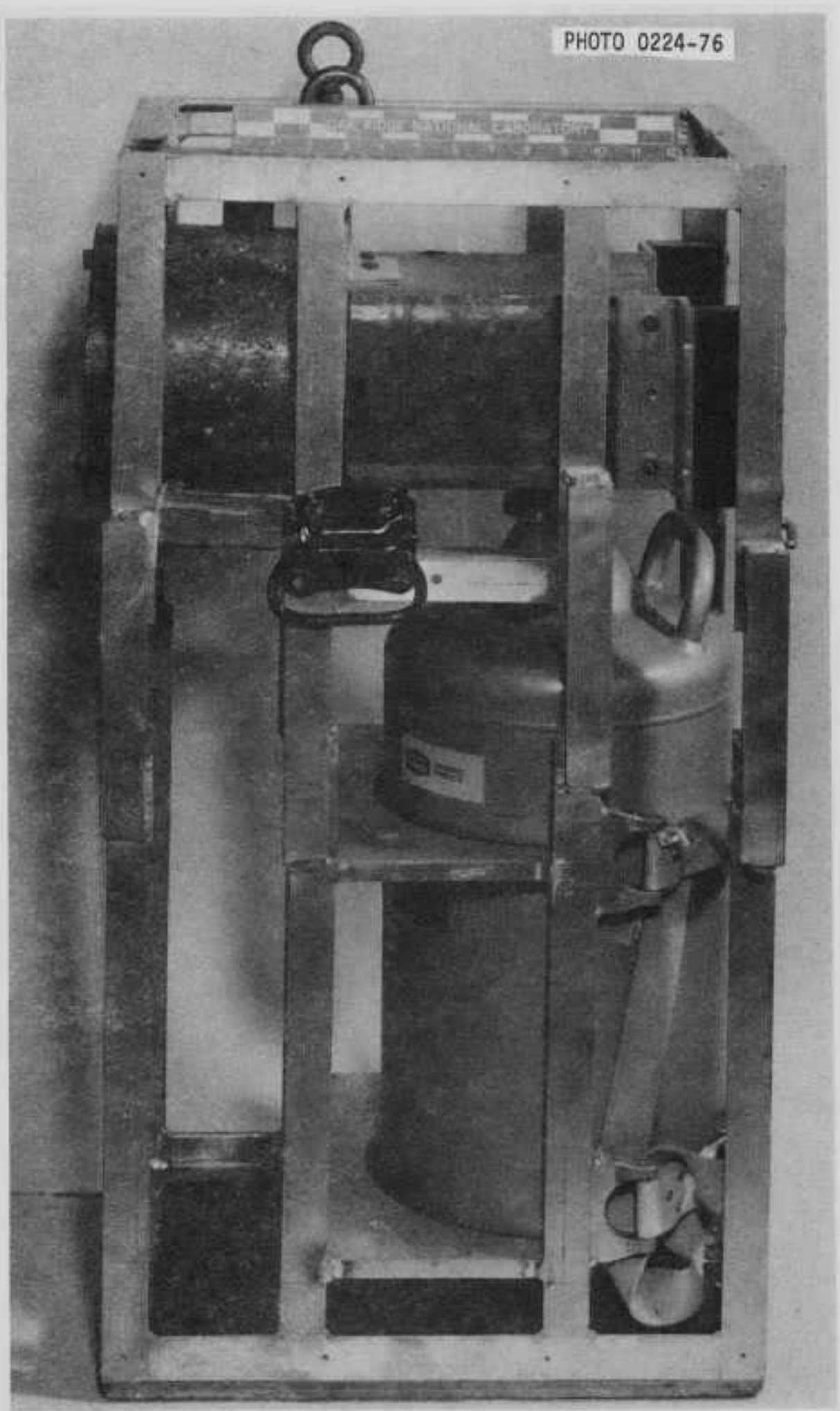

Fig. 2.2-2. Shielded detector system used to acquire spectra of Peach Bottom duct. 
has a relatively low energy, lowered the count rate, prevented significant degradation of the detector resolution, and maintained a higher detection sensitivity for low energy photons from duct activities (e.g., the 364-keV gamma ray of ${ }^{13} I_{I}$ ).

Because of various circumstances that limited the placement of the detector at selected locations on the duct, it was necessary to provide two detector-duct counting configurations. Configuration No. 1, shown schematically in Fig. 2.2-3, illustrates the details of the measurements. Shown are the active Ge(Ii) detector, cryostate Dewar, collimator shield, and a cross section of the duct. The duct had a 1.11-cm (7/16-in.) carbon steel wall and was wrapped with $7.6 \mathrm{~cm}$ (3 in.) of thermal insulation. In this counting configuration, gamma rays from restricted regions on the duct nearest to and farthest from the detector traversed the steel wall, the thermal insulation, the opening in the face of the collimator shield, and intersected the active $\mathrm{Ge}(\mathrm{Ii})$ detector. In counting configuration No. 2 (not shown), the detector was placed under the duct, and gamma rays went through the side opening in the collimator shield. In measurements made with configuration 1 , the face of the collimator shield was nominally in contact with the duct insulation; when configuration 2 was used, the collimator shield was between 2.5 and $10 \mathrm{~cm}$ from the insulation. Scale drawings (not shown) indicated that in no case did the subtended region on the duct exceed the duct diameter.

Except for those spectra taken in 1972, measurements were begun within three to five days following reactor shutdown. After such short time intervals, when the ambient temperature ranged from 27 to $35^{\circ} \mathrm{C}$, it was necessary to place a thermal radiation shield around the detector to prevent any damage by excessive temperatures. This shielding was provided by wrapping the detector cryostat with a few layers of polyrurethane foam and aluminum foil.

Spectra acquired at Peach Bottom were recorded on paper and/or magnetic tape and processed at ORNL to obtain specific surface activities after counting efficiencies were determined. Counting efficiencies for the primary circuit piping were determined with a mock-up that simulated the 
ORNL DWG. 76-111

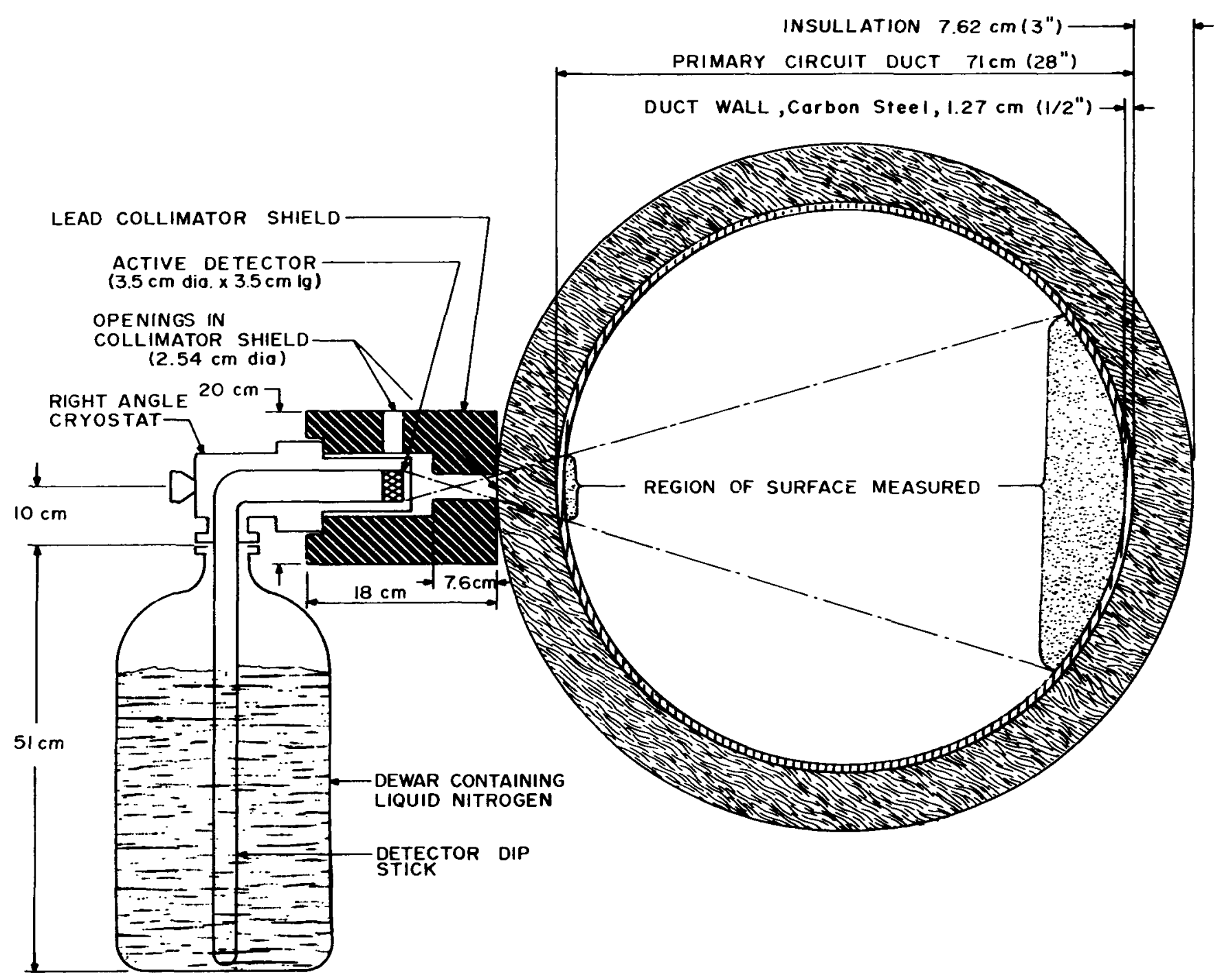

Fig. 2.2-3. Counting configuration No. 1 of detector and Peach Bottom duct. 
physical size of the duct. The mock-up was uniformily coated with ${ }^{140}$ La and was counted with the same detector-duct configurations used at Peach Bottom. The specific surface activity of the ${ }^{140}$ La was then accurately measured and used, along with the count rates observed on the mock-up, to compute counting efficiencies. The details of the counting efficiency calibrations are discussed in Appendix A.

Specific surface activities, $A\left(\mu \mathrm{Cl} / \mathrm{cm}^{2}\right)$, of radionuclides on the duct were computed according to the expression:

$$
\mathrm{A}\left(\mu \mathrm{Ci} / \mathrm{cm}^{2}\right)=\frac{\mathrm{R}}{\mathrm{BR} \cdot \mathrm{EF}(\mathrm{E}) \cdot 3 \cdot 7 \mathrm{E} 4},
$$

where

$$
\begin{aligned}
\mathrm{R}= & \text { net count rate of a photopeak, } \mathrm{C} / \mathrm{S}, \\
\mathrm{BR}= & \text { branching ratio of the gamma ray (photons emitted per } \\
& \text { disintegration), } \\
\mathrm{EF}(\mathrm{E})= & \text { counting efficiency for the gamma ray expressed as a } \\
& \text { function of gamma energy, }
\end{aligned}
$$

and the factor

$$
3.7 \mathrm{E} 4=\text { converts the activity to } \mu \mathrm{Ci} \text {. }
$$

The effects of gamma-ray attenuation in the duct walls and thermal insulation surrounding the duct were incorporated in the counting efficiency as explained in Appendix A.

\subsection{Data Sumnary of Primary Loop Gamma Scans}

The primary coolant piping was gamma-scanned on four ${ }^{*}$ occasions durIng the life of Peach Bottom Core 2 at the locations specified in Fig. 2.1-1 and in Table 2.1-1. Plateout activities determined in these four tests are summarized in Tables 2.3-1 to 2.3-4. Each test was conducted during a shutdown period, and the data was corrected back to the time of shutdown. The relevant dates are listed for each of the tests. Tables 2.3-1 and 2.3-2 list revised values that were originally given in ref. 3; Table 2.3-3 is an augmented version of the data presented in ref. 4, whereas Table 2.3-4

\footnotetext{
*An additional single spectrum at location 5 was acquired on June 10, 1974.
} 
Table 2.3-1. Coolant duct activities as of Apri1 22, 1971

(Data acquired May 10 to $14,1971^{a}$ )

\begin{tabular}{|c|c|c|c|c|c|c|c|c|c|c|c|c|c|}
\hline \multirow[b]{2}{*}{ Location } & \multicolumn{13}{|c|}{$\mu \mathrm{Ci} / \mathrm{cm}^{2}$} \\
\hline & $54 \mathrm{Mn}$ & $59_{\mathrm{Fe}}$ & ${ }^{60} \mathrm{Co}$ & ${ }^{65} \mathrm{zn}$ & ${ }^{103} \mathrm{Ru}$ & ${ }^{106} \mathrm{Ru}$ & $110 \mathrm{~m}_{\mathrm{Ag}}$ & $131_{I}$ & $13^{4} \mathrm{Cs}$ & $137_{\mathrm{Cs}}$ & $140 \mathrm{Ba}$ & ${ }^{144} \mathrm{Ce}$ & ${ }^{154} \mathrm{Eu}$ \\
\hline 1 & 0.0030 & 0.0063 & 0.0066 & 0.012 & $\leq 0.002$ & $\leq 0.002$ & 0.011 & $\leq 0.008$ & 0.19 & 0.58 & 0.0033 & 0.17 & $\leq 0.0005$ \\
\hline 4 & 0.0026 & 0.0019 & 0.00057 & 0.011 & $\leq 0.004$ & $\leq 0.02$ & 0.010 & $\leq 0.02$ & 0.21 & 0.69 & 0.0030 & 0.02 & $\leq 0.0007$ \\
\hline 5 & 0.0021 & 0.0010 & 0.00042 & 0.011 & $\leq 0.003$ & $\leq 0.02$ & 0.0087 & $\leq 0.01$ & 0.21 & 0.62 & 0.0031 & 0.01 & $\leq 0.0004$ \\
\hline 6 & 0.0018 & 0.0071 & 0.0053 & 0.0039 & $\leq 0.007$ & $\leq 0.03$ & 0.0034 & $\leq 0.008$ & 0.079 & 0.25 & 0.0022 & 0.02 & $\leq 0.0004$ \\
\hline 7 & 0.0013 & 0.0071 & 0.0080 & 0.0018 & 0.0003 & 0.0043 & 0.0026 & $\leq 0.001$ & 0.046 & 0.13 & 0.0029 & 0.028 & 0.0004 \\
\hline
\end{tabular}

${ }^{9} I_{\mathrm{Cr}},{ }^{136} \mathrm{Cs}$, and ${ }^{233} \mathrm{~Pa}$ were not detected. Decay time was too long to permit upper limits to be estimated for ${ }^{132}$ Te. 
Table 2.3-2. Coolant duct activities as of January $6,1972^{a}$

(Data acquired April 24 to 28, 1972)

\begin{tabular}{|c|c|c|c|c|c|c|c|c|c|c|c|}
\hline \multirow[b]{2}{*}{ Location } & \multicolumn{11}{|c|}{$\mu \mathrm{Ci} / \mathrm{cm}^{2}$} \\
\hline & $54 \mathrm{Mn}$ & ${ }^{59} \mathrm{Fe}$ & ${ }^{60} \mathrm{Co}$ & $65_{\mathrm{zn}}$ & ${ }^{103} \mathrm{Ru}$ & ${ }^{106} \mathrm{Ru}$ & $110 \mathrm{~m}_{\mathrm{Ag}}$ & $134 \mathrm{Cs}$ & $137_{\mathrm{Cs}}$ & $144 \mathrm{Ce}$ & $154_{\mathrm{Eu}}$ \\
\hline 1 & 0.0015 & 0.0028 & 0.0028 & 0.0045 & $\mathrm{ND}^{\mathrm{b}}$ & ND & 0.0033 & 0.079 & 0.27 & 0.020 & $\leq 0.00008$ \\
\hline 3 & 0.0011 & 0.0011 & 0.00077 & 0.0032 & $\leq 0.0008$ & $\leq 0.004$ & 0.0020 & 0.064 & 0.22 & 0.006 & $\leq 0.00008$ \\
\hline 4 & 0.0012 & 0.0010 & 0.00059 & 0.0039 & $\leq 0.0005$ & $\leq 0.002$ & 0.0027 & 0.064 & 0.23 & 0.004 & $\leq 0.00006$ \\
\hline 5 & 0.00059 & $\leq 0.002$ & 0.007 & 0.0019 & $\leq 0.002$ & $\leq 0.006$ & 0.0014 & 0.052 & 0.17 & 0.004 & $\leq 0.0002$ \\
\hline 6 & 0.006 & 0.0092 & 0.0010 & 0.0038 & $\leq 0.003$ & $\leq 0.009$ & 0.0033 & 0.058 & 0.22 & 0.017 & 0.0005 \\
\hline 8 & 0.00096 & 0.0035 & 0.0022 & 0.0022 & $\leq 0.002$ & $\leq 0.005$ & 0.0010 & 0.032 & 0.12 & 0.012 & $\leq 0.0003$ \\
\hline
\end{tabular}

Decay time was too long to permit detection of radionuclides with half lives of about one month or less.

$\mathrm{b}_{\text {Signifies not detected. }}$ 
Table 2.3-3. Coolant activities as of September 14, 1973 (data acquired Septr-rber 19 to 21, 1973)

$$
\left(\mu \mathrm{Ci} / \mathrm{cm}^{2}\right)
$$

\begin{tabular}{|c|c|c|c|c|c|c|c|c|c|c|c|c|c|c|c|c|c|c|}
\hline Location & CR51 & $2 E R^{a}$ & MN 54 & \%ER & FE59 & \%ER & $\mathrm{CO} 60$ & \%ER & $\mathrm{zN} 65$ & \%EP & RU1 03 & \%ER & RU1 06 & \%ER & $A G 110$ & \%ER & 1131 & \\
\hline 1 & $1.90 E-02$ & $*^{b}$ & $1.73 E-03$ & 12.0 & $1.04 E-03$ & 18.0 & $3.94 E-03$ & 3.0 & $4.50 E-03$ & 6.7 & $2.00 \mathrm{E}-03$ & * & $1.00 E-02$ & * & $55 E-03$ & 15.0 & $8.85 E-03$ & \\
\hline 3 & $2.04 E-02$ & 30.5 & $1.68 E-03$ & $7 \cdot 1$ & $4 \cdot 61 E-04$ & 19.0 & $1.07 E-03$ & $4 \cdot 5$ & $1.81 E-03$ & $8 \cdot 5$ & $1.00 E-03$ & * & $7.00 E-03$ & * & $1.10 E-03$ & 12.5 & $4.80 E-03$ & \\
\hline 4 & $1.20 E-02$ & * & $1.41 E-03$ & 10.0 & $3.10 E+01$ & * & $7.96 E-04$ & $7 \cdot 4$ & $2.56 E-03$ & 8.0 & $1.00 E-03$ & * & $9.00 E-03$ & * & $1.77 E-03$ & 12.2 & 2. $46 E-03$ & \\
\hline 5 & $5 \cdot 91 E-03$ & 31.0 & $7 \cdot 22 E-04$ & 6.0 & $2.47 E-04$ & 14.0 & $7.94 E-04$ & 2.4 & $1.59 E-03$ & 4.0 & $3.00 \mathrm{E}-04$ & * & $2.00 E-03$ & * & $5 \cdot 67 E-04$ & $8 \cdot 5$ & $1.80 E-03$ & \\
\hline 6 & $1.60 E-02$ & 28.0 & $1.08 E-03$ & 8.7 & $1.08 E-03$ & $9 \cdot 5$ & $3.94 E-03$ & 1.7 & $1.33 E-03$ & 10. & $8.00 E-04$ & * & $5.00 E-03$ & * & $9.76 \mathrm{E}-04$ & 13. $c$ & $1.83 E-03$ & \\
\hline 7 & 2. $73 E-02$ & 10.0 & $3.21 E-03$ & 2.8 & $5 \cdot 56 E-03$ & 2.0 & $4 \cdot 50 E-02$ & 0.2 & $1.79 E-03$ & $7 \cdot c$ & $5 \cdot 10 E-03$ & $4 \cdot 4$ & $9 \cdot 30 E-03$ & 19.0 & $2.76 E-03$ & $5 \cdot 0$ & 3. $30 E-03$ & \\
\hline 8 & $3.48 E-02$ & 17.0 & $1.42 E-03$ & 11.0 & $2.83 E-03$ & 6.8 & $4 \cdot 33 E-03$ & $2 \cdot 6$ & $2 \cdot 88 \mathrm{E}-03$ & $8 \cdot 2$ & $.00 E-04$ & * & $3.00 \mathrm{E}-03$ & * & $5.69 E-04$ & 29.0 & 03 & \\
\hline
\end{tabular}


Table $3.3-3$. Continued

\begin{tabular}{|c|c|c|c|c|c|c|c|c|c|c|c|c|c|c|c|c|c|}
\hline Location & TE 132 & $\% E P$ & $\operatorname{css} 134$ & \%ER & $\operatorname{cs} 136$ & $\% E R$ & $c \subseteq 137$ & $\% E R$ & BA140 & $\% E F$ & CE 144 & $\% E R$ & EU154 & 2ER & PA233 & $\% \mathrm{ER}$ & $\operatorname{CS} 137 / \operatorname{CS} 134$ \\
\hline$I$ & $5.50 E-03$ & $*$ & $3.89 E-01$ & 0.3 & $2.23 E-02$ & 3.7 & 5. $40 E-01$ & 0.2 & $2 \cdot 18 E-03$ & 3.8 & $6 \cdot 44 E-02$ & 36.0 & 5. $00 E-04$ & * & $1.40 E-31$ & * & $1.38 E+00$ \\
\hline 3 & 2. $29 E-03$ & $*$ & $2.93 E-01$ & 0.2 & $1 \cdot 42 E-02$ & $3 \cdot 2$ & $4 \cdot 69 E-01$ & 0.2 & $1.31 E-03$ & $3 \cdot 3$ & 2. $32 E-02$ & * & 3. $10 E+01$ & * & $1.10 E-01$ & * & $1.60 E+00$ \\
\hline 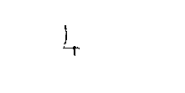 & 3. $43 E-03$ & * & $3.39 E-01$ & 0.3 & $1.57 E-02$ & 3.8 & $5.08 E-01$ & 0.2 & $1.56 E-03$ & $3 \cdot 8$ & 3. $93 E-02$ & * & $1.00 \mathrm{E}-04$ & * & $1 \cdot 30 E-01$ & $*$ & $1.49 \pm+00$ \\
\hline 5 & $1.47 E-03$ & * & 5. $23 E-02$ & 0.2 & $1.40 E-02$ & $1 \cdot 4$ & $1.28 E-01$ & 0.2 & $1 \cdot 83 E-03$ & $1 \cdot 1$ & $1 \cdot 11 E-02$ & 46.0 & $3.00 E-04$ & * & $1.00 E-02$ & 28.0 & $2.19 E+00$ \\
\hline 6 & 2. $70 E-03$ & * & $2.02 E-01$ & 0.2 & $1 \cdot 49 E-02$ & 2.9 & $3.03 E-01$ & 0.2 & $2.18 E-03$ & $2 \cdot 2$ & $2 \cdot 35 E-02$ & * & $3.00 E-04$ & * & $9.50 E-02$ & * & 1. $50 E+00$ \\
\hline 7 & $2.80 E-03$ & * & $2.89 E-01$ & 0.1 & $2.20 E-02$ & 1.6 & $3.96 \mathrm{E}-01$ & 0.1 & $8.36 E-03$ & 0.6 & $9.50 \mathrm{E}-03$ & 18.0 & $5.00 E-04$ & * & $5 \cdot 10 \mathrm{E}-02$ & $1 \cdot 2$ & $1 \cdot 37 E+00$ \\
\hline 8 & $3.30 E-03$ & * & $1.71 E-01$ & 0.4 & $1.27 E-02$ & 4.9 & $2.47 E-01$ & 0.3 & $2 \cdot 49 E-03$ & $3 \cdot 2$ & $3.49 E-02$ & * & $2.00 E-04$ & * & $7.10 E-02$ & * & $1.44 E+00$ \\
\hline
\end{tabular}

$\partial_{\text {d/W }} \mathrm{R}$ denotes l-sigma counting statistics error.

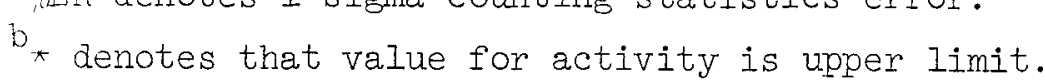


Table 2.3-4. Coolant duct activities as of October 31, 1974 (data acquired November 4 to 8, 1974)

$$
\left(\mu \mathrm{Ci} / \mathrm{cm}^{2}\right)
$$

\begin{tabular}{|c|c|c|c|c|c|c|c|c|c|c|c|c|c|c|c|c|}
\hline Location & CR5I & \%ER ${ }^{a}$ & MN 54 & $\% E R$ & FE59 & $\% E R$ & C060 & \%ER & $\mathrm{ZN} 65$ & zER & $A G 110$ & \%ER & 1131 & gER & $T E 132$ & \%ER \\
\hline 1 & 3. $40 E-01$ & $*^{b}$ & $4.87 E-03$ & * & $4 \cdot 22 E-03$ & * & $8.84 E-03$ & 6.6 & $8 \cdot 32 E-03$ & 24.0 & $3.93 E-03$ & * & $5.05 E-02$ & * & $5 \cdot 20 E-02$ & * \\
\hline 2 & $1.05 E-01$ & * & $1.56 E-03$ & * & $1.78 E-03$ & * & $1.51 E-03$ & 13.0 & $1.69 E-03$ & * & $1.97 E-03$ & * & $1.93 E-02$ & * & $2.80 E-02$ & * \\
\hline 3 & $1.40 E-01$ & * & $1.82 E-03$ & $25 \cdot c$ & $6.55 E-04$ & $35 \cdot c$ & $5 \cdot 24 E-04$ & $9 \cdot 5$ & $1.14 E-03$ & $20 \cdot 3$ & 4. $73 E-04$ & * & $2.97 E-02$ & * & $8.40 E-03$ & 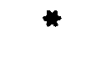 \\
\hline 4 & $1.86 E-01$ & * & $1.31 \mathrm{E}-03$ & $32 \cdot 0$ & $8.71 E-04$ & * & $7 \cdot 11 E-04$ & 12.8 & $1.64 E-03$ & $24 \cdot 0$ & $1.04 E-03$ & * & $3.84 E-02$ & * & $8.20 E-03$ & * \\
\hline $5^{a}$ & & & & & & & & & & & & & & & & \\
\hline 5 & $1.89 E-01$ & * & $1.63 E-03$ & * & $1.24 E-03$ & * & $1.86 E-03$ & $7 \cdot 8$ & $1.29 E-03$ & * & $1.74 E-03$ & $*$ & $1.10 E-01$ & * & $1.40 E-02$ & * \\
\hline 6 & $2.08 E-02$ & * & $2 \cdot 14 E-03$ & $14 \cdot 0$ & $3.41 E-03$ & $10 \cdot 3$ & $1 \cdot 19 E-02$ & $1 \cdot 0$ & $2 \cdot 78 E-03$ & 12.6 & $1.77 \mathrm{E}-03$ & $20 \cdot 3$ & $2.05 E-02$ & * & $2.90 E-02$ & * \\
\hline 7 & $3.30 E-01$ & * & $7.89 E-03$ & * & $8 \cdot \angle: 0 E-03$ & * & $7 \cdot 16 \mathrm{E}-02$ & $2 \cdot 4$ & $8 \cdot 17 E-03$ & * & $7 \cdot 28 E-03$ & * & $7.04 E-02$ & * & $1.30 E-01$ & \\
\hline 8 & $1.60 E-01$ & * & $4 \cdot 34 E-03$ & * & $4 \cdot 76 E-03$ & * & $7 \cdot 98 E-03$ & $7 \cdot 9$ & $8 \cdot 97 E-03$ & $25 \cdot 0$ & $4.79 E-03$ & * & $2.76 \mathrm{E}-02$ & * & 5. $10 E-02$ & * \\
\hline
\end{tabular}


Table 2.3-4. Continued

\begin{tabular}{|c|c|c|c|c|c|c|c|c|c|c|c|c|c|c|c|}
\hline Location & $\operatorname{cs} 134$ & \%ER & $\operatorname{cs} 136$ & zER & $\operatorname{cs} 137$ & FER & BA 140 & \%ER & $C E 144$ & $\% E R$ & EU1 54 & \%ER & PA233 & \%ER & $\operatorname{cs} 137 / \operatorname{Cs} 134$ \\
\hline 1 & $3 \cdot 15 E+00$ & 0.2 & $1 \cdot 26 E-02$ & $41 \cdot 0$ & $2 \cdot 63 E+00$ & 0.2 & $1 \cdot 22 E-03$ & 27.0 & $2.30 E-01$ & * & $3.77 E-03$ & * & $6.00 E-01$ & * & $8.34 E-01$ \\
\hline 2 & $1 \cdot 78 E+00$ & $0 \cdot 3$ & $6 \cdot 25 E-03$ & 13.0 & $1 \cdot 38 E+00$ & 0.3 & $6.85 E-04$ & 15.0 & $2 \cdot 13 E-01$ & * & $9.06 E-04$ & * & $3.00 E-01$ & * & 7. $75 E-01$ \\
\hline 3 & $1 \cdot 75 E+00$ & 0.1 & $6 \cdot 11 E-03$ & $7 \cdot 4$ & $1 \cdot 45 E+00$ & 0.1 & $4 \cdot 21 E-04$ & $6 \cdot 0$ & $1.43 E-01$ & $21 \cdot 0$ & $3.71 E-04$ & * & $2.59 E-01$ & * & $8 \cdot 28 E-01$ \\
\hline 4 & $1.89 E+00$ & 0.1 & $5 \cdot 38 E-03$ & 14.0 & $1 \cdot 52 E+00$ & 0.2 & $4 \cdot 23 E-04$ & $12 \cdot 0$ & $1.10 E-01$ & * & $6.14 \mathrm{E}-04$ & * & $2.10 E-01$ & * & $8.04 E-01$ \\
\hline $5^{a}$ & $7.60 E-01$ & $1 \cdot 0$ & & & $6.60 E-01$ & 1.0 & & & & & & & & & $8.68 E-01$ \\
\hline 5 & $1 \cdot 21 E+00$ & 0.3 & $4.94 E-03$ & 11.0 & $9.60 E-01$ & 0.3 & $9 \cdot 56 E-04$ & $7 \cdot 1$ & $1.90 \mathrm{E}-01$ & * & $1.00 E-03$ & * & $1.34 E+00$ & * & 7. $93 E-01$ \\
\hline 6 & $1 \cdot 89 E+00$ & 0.1 & $1 \cdot 35 E-02$ & $6 \cdot 2$ & $1 \cdot 72 E+00$ & 0.1 & $2.07 E-03$ & $2 \cdot 8$ & $5.85 E-02$ & * & 5. $15 E-04$ & * & $2.63 E-01$ & * & $9.10 \mathrm{E}-01$ \\
\hline 7 & $2.40 E+00$ & 0.4 & $1.87 E-02$ & * & $2.00 E+00$ & 0.5 & $1 \cdot 25 E-03$ & * & $6.00 E-01$ & * & $7.06 E-03$ & * & $6.50 E-01$ & * & $8.33 E-01$ \\
\hline 8 & $1.86 E+00$ & 0.3 & $2.62 E-02$ & $21 \cdot 0$ & $1.59 E+00$ & 0.4 & $2 \cdot 92 E-03$ & $12 \cdot 7$ & $3.70 \mathrm{E}-01$ & * & 3. $98 E-03$ & * & $3.10 \mathrm{E}-01$ & * & 8. $54 E-01$ \\
\hline
\end{tabular}

$a_{\% F R}$ denotes 1-sigma counting statistics error.

$b_{*}$ denotes that value for activity is upper limit. 
shows the results of the most recent gamma scan taken approximately two weeks after the final shutdown of the reactor. A single data point obtained at location 5 in May, 1974, is also recorded on Table 2.3-4.

It is worth noting that iodine photopeaks were observed only in the Sept. 1973 scan data, and only upper detection limit activities were recorded for the 1971 and 1974 scans. The 1972 scan was acquired far too long after reactor shutdown to offer any possibility of detecting the 8-day ${ }^{13} I_{I}$ photopeak.

\subsection{Discussion of Primary Loop Gamma Scan Data}

Figure 2.4-I summarizes some interesting features of the large duct gamma-scan data. Note that the initial period of Core 2 operation saw a diminution of ${ }^{137}$ Cs activity on the cold duct, evidently, as a result of the removal of large deposits left from Core 1 operation. Further evidence that the early cesium activity was largely left from Core $I$ is offered by the observed initial increase in the ${ }^{137} \mathrm{Cs} /{ }^{134} \mathrm{Cs}$ ratio. "OId" Cs would be characterized by a lower-than-normal quantity of ${ }^{134} \mathrm{Cs}$ by virtue of its shorter half-life. Thus, the observed drop in the ratio during Core 2 operation after 1972 indicates that "old" Cs was being replaced by $\mathrm{Cs}$ generated in Core 2. The approximate fraction of ${ }^{137} \mathrm{Cs}$ deposited on the cold ducts, relative to total core inventory, shows a similar drop from $2.2 \times 10^{-5}$ initially observed for the Apr. 1971 data, to approximately $0.4 \times 10^{-5}$ for the Jan. 1972 and Sept. 1973 measurements, subsequently followed by an elevation to $2.5 \times 10^{-5}$ at the end-of-life.

Although gamma spectra of the concentric duct were acquired, there is no unambiguous way to apportion photopeak count rates to the three surfaces of the duct, that is, the inner and outer surfaces of the inner duct and the inner surface of the outer duct. In addition to the possibility that these surfaces had different levels of activites on them, the derivation of activity levels is complicated by the complex situation regarding the attenuation of gamma rays in traversing the duct walls to reach the detector. Although the radionuclides found on the concentric duct were the same as those observed on the cold duct, the observed count rates were 
ORNL OWG 75-15012RI

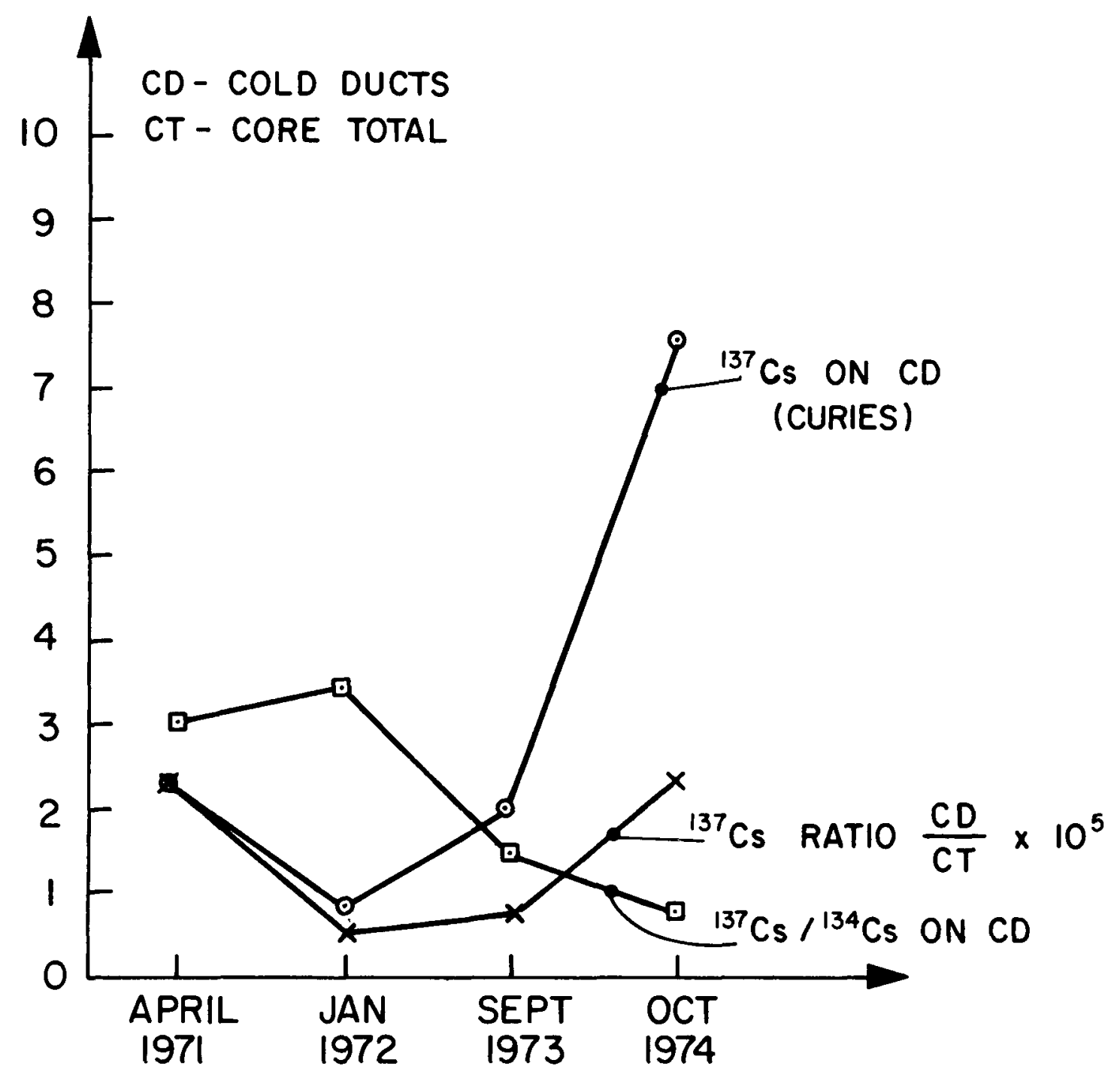

Fig. 2.4-1. Cesium activity on Peach Bottom cold ducts. 
considerably lower than the count rates at nearby locations on the cold duct. Table 2.4-1 gives values for the ${ }^{137} \mathrm{Cs}$ count rates observed on the concentric duct and at locations 7 and 8 on the cold duct in 1971, 1972 , and 1973.

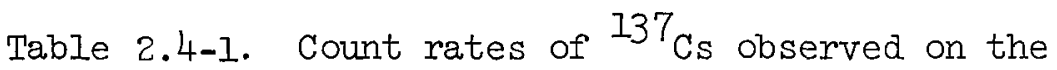
concentric duct and cold duct

\begin{tabular}{lcccc}
\hline & \multicolumn{3}{c}{ Observed count rate of ${ }^{137} \mathrm{Cs}$ (counts/sec) } \\
\cline { 2 - 4 } Location & 1971 & 1972 & 1973 \\
\hline Concentric duct & 5 & 6 & 6 \\
Cold duct & $31^{\mathrm{a}}$ & $16^{\mathrm{b}}$ & $30^{\mathrm{b}}$ \\
\hline $\mathrm{a}_{\text {Location } 7 .}$ & & & \\
$\mathrm{b}_{\text {Location } 8 .}$ & & &
\end{tabular}

One may, in principle, estimate the surface activity inside the hot duct if the activity level on the annular surfaces outside the hot duct were accurately known. For this purpose, it may be reasonable to assume the same values that were observed for near upstream locations on the cold duct, that is, locations 7 and 8 . However, an additional complicating factor renders any attempt to assess the hot duct activity in this fashion as futile. The concentric duct readings were taken at a location where wall thicknesses varied, that is, in a conical transition region leading to the hot valve. Whereas nominal concentric duct wall thicknesses are $3 / 8$ in. and $3 / 4$ in. for the inner and outer duct, respectively, wall thicknesses in this transition zone ranged about $1-1 / 4 \mathrm{in}$. for both ducts. These greater wall thicknesses explain the low readings obtained at this location, which are given in Table 2.4-1. It is not possible to derive hot duct activities by difference because of the uncertain value for the attenuation factor. 
References for Section 2

1. Peach Bottom Atomic Power Station FHSR, Part C, Philadelphia EIectric Company, Section II-D, Docket 50171-3 (Mar. 1964).

2. F. F. Dyer, R. P. Wichner, W. J. Martin, L. L. Fairchild, R. J. Kedl, and H. J. de Nordwall, Postirradiation Examination of Peach Bottom HTGR Driver Fuel Element EO6-01, ORNL-5126 (1976).

3. GCRP Annu. Prog. Rep. Period Ending 12/31/72, ORNL-4911 (Mar. 1974 ).

4. GCRP Annu. Prog. Rep. Period Ending 12/31/73, ORNL-4975 (Apr. 1976 ).

3. PRIMARY COOLANT SAMPLERS FOR GASEOUS RADIONUCLIDES

AND PARTICUTATES

\subsection{Description of Experiment}

Direct access to the primary coolant flow was attained through gassampling tubes incorporated in the reactor outlet valve upstream from the steam generator and via the thermowell downstream from the steam generator indicated in Fig. 2.1-1, Sect. 2. A coolant sampler, the general external features of which are shown in Fig. 3.1-1, extended. into the sampler port so that the end of the barrel coincided with the pipe wall and the nozzle pointed directly upstream. Coolant samples were withdrawn at a rate such that the nozzle and coolant velocities were equal in order to obtain a representative sample of the particulates entrained in the gas flow. The sampling periods ranged from $\sim 1700$ to $\sim 2700 \mathrm{hr}$ for the ORNL devices.

The experiments were designed to separate the following radionuclides from the helium sample: (1) gaseous, condensible radionuclides via deposition on the inner surfaces of a set of diffusion tubes, (2) radionuclides borne by particulates by retention on filters downstream from the diffusion tubes (later a five-stage particle impactor was included in the downstream sampler for size classification), (3) noncondensible, gaseous radionuclides were retained on charcoal absorbers downstream from the filters. Of considerable interest was the possible detection of 


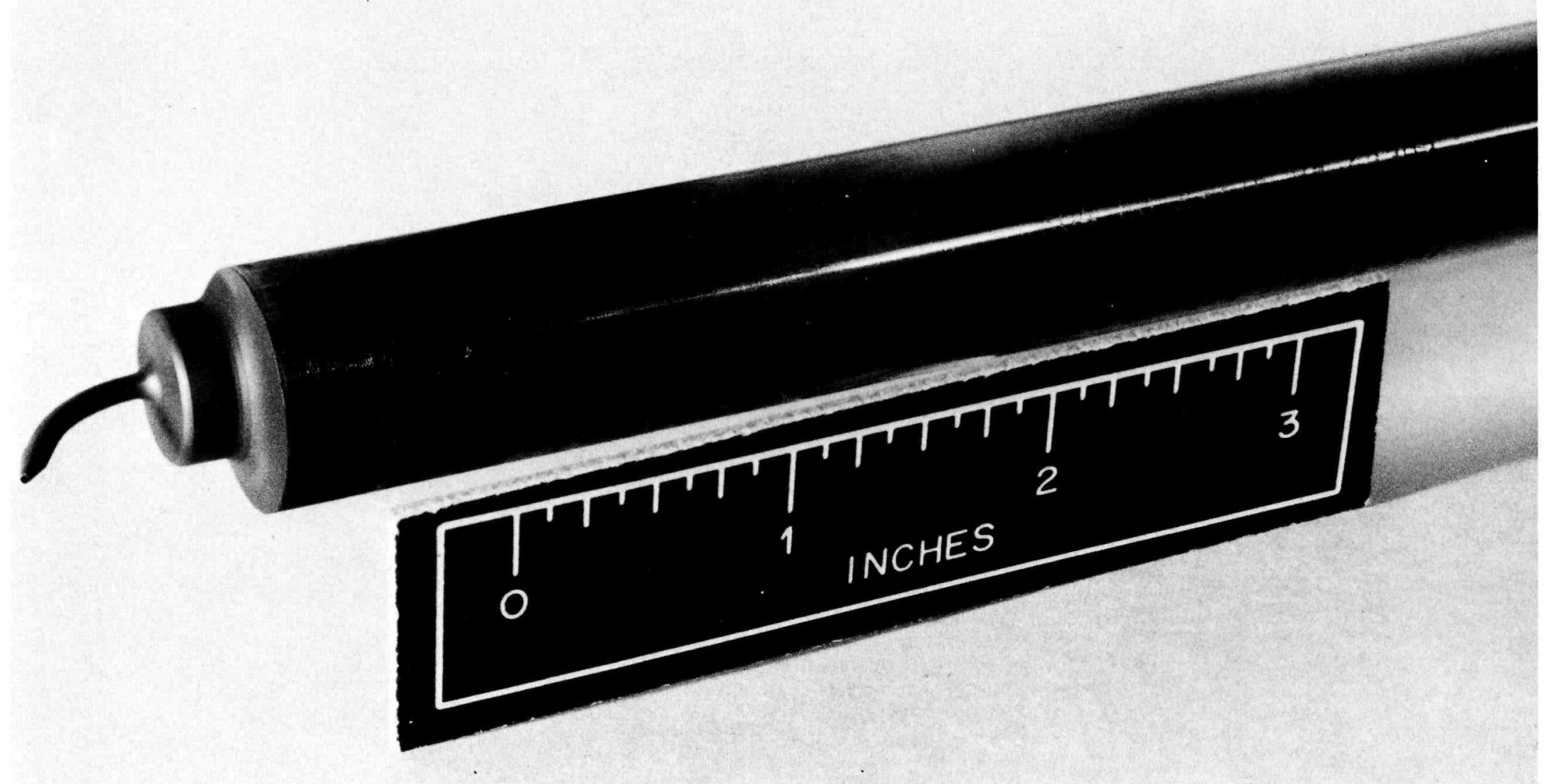

Fig. 3.1-1. Upstream sampler (CA), barrel and nozzle. 
organic iodine on the absorbers; the daughters of noble geses that had passed through the diffusion tubes and filters were seen here also.

A descriptive sumbry of the seven sampling experinents performed. auring Core 2 operation appears in Table 3.1-1. The table indicates the sampler code-letter designations used to identify the experiment, the location of the sampler, active sampling dates and total operation time, total sample passed, estimeted inlet and deposition conditions, as well as some brief descriptive notes on the type of sampler employed. The final column relates each sampling experiment to a descriptive figure appearing in this section or to some other pertinent reference.

As Table 3.1-1 indicates, the sarpling experiments varied somewhat iuring the course of the program. The first ORNL sampler, designated cl and located downstream from the steen generator, 1s illustrated in Fíg. 3.1-2. The sampler nozzle was formed from a 1/8-in. stainlesa steel tube w1th a 0.04l-in. (0.10-cm) II. Deteils for the nozzle, shown in Fig. 3.1-3, were identical for all the downstream samplers. The Cl sampler barrel contained three silver diffusion tubes, each having a $0.166-i n$. (0.422-cm) oD with a $0.005-i n .(0.13-1 \mathrm{~m})$ wall thickneas and were $55.1 \mathrm{~cm}$ long. The sampler barrels, in all cases, sere set in a verticle position.

The asserbly of the first upstresm sampler, $C A$, is shown in Fig. 3.1 .4 with the nozzle and diffusion tube detajs shom in Fig. 3.I-5. Note, the upstream samplers employed a sanewhat suller nozzle than the downstream samplers. Figure 3.1-6 illustrates the filter unt used with sampler CA for the first portion of the experiment, which is designated as run $\mathrm{CA}-1$ in Table 3.1-1. The filter unit was located approximately 2 in from the diffusion tube assembly; hence, the possiblitity exists for particulate losses to have occurred in this line.

The filter untt used with the sampler $\mathrm{CA}$ was replaced after $1700 \mathrm{hr}$ of operation by the unit shown in Fis. 3.1-7, and the remainder of the run was termed $\mathrm{CA}-2$ for the purpose of identification. The second filter unit used wth the $C A$ samplex contained a charcoal adsorber downstream from a fiberglass absolute filter. Adjitionally, a sintered silver membrane filter, manufactured by Selas Flotronics with an averaye pore aize of $0.2 \mu \mathrm{Il}$, was aituated directly upstream from the absolute filter. 
Table 3.1-1. Surmary of Core 2 coolant sampler experiments

\begin{tabular}{|c|c|c|c|c|c|c|c|c|c|}
\hline 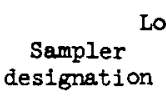 & $\begin{array}{l}\text { cation relat } \\
\text { to steam } \\
\text { generator }\end{array}$ & $\begin{array}{l}\text { ive } \\
\text { Service dates }\end{array}$ & $\begin{array}{l}\text { Operating } \\
\text { time } \\
\text { (hr) }\end{array}$ & $\begin{array}{l}\text { Coolant } \\
\text { flowrate } \\
(\mathrm{kg} / \mathrm{hr})\end{array}$ & $\begin{array}{l}\text { Coolant } \\
\text { throughput } \\
\quad(\mathrm{kg})\end{array}$ & $\begin{array}{l}\text { Inlet } \\
\text { temperature } \\
\left({ }^{\circ} \mathrm{C}\right)\end{array}$ & $\begin{array}{l}\text { Diffusion tube } \\
\text { temperature } \\
\left({ }^{\circ} \mathrm{C}\right)\end{array}$ & Sampler components & Comments \\
\hline CG & Upstream & (?) & 6627 & 0.047 & 309 & 680 & $\sim 350$ & $\begin{array}{l}\text { Three stainless steel diffusion } \\
\text { tubes, cellulose filter, char- } \\
\text { coal absorber for } 1 \text {. }\end{array}$ & $\begin{array}{l}\text { Design and operation by CAC. } \\
\text { Tubes } 0,1 \text {, and } 2 \text { had } 13,29 \text {, } \\
\text { and } 53 \% \text { of the total fiow, } \\
\text { respectively. }\end{array}$ \\
\hline $\mathrm{Cl}$ & Downstreem & $7 / 29 / 71-12 / 14 / 71$ & 12757 & 0.21 & 579 & 300 & $\sim 130$ & $\begin{array}{l}\text { Three silver diffusion tubes, } \\
\text { silver membrane filter, fiber- } \\
\text { glass absolute filter, }\end{array}$ & Figs. $3 \cdot 1-2$ and $3 \cdot 1-3$. \\
\hline $\mathrm{CA}-1$ & Upstream & $9 / 13 / 72-11 / 24 / 72$ & $2 \quad 1727$ & 0.12 & 207 & 680 & $\sim 350$ & $\begin{array}{l}\text { Six stainless steel diffusion } \\
\text { tubes, silver membrane filter. }\end{array}$ & Figs. $3.1-4,3 \cdot 1-5,3.1-6$ \\
\hline $\mathrm{CA}-2$ & Upstream & $1 / 17 / 73-5 / 17 / 73$ & 2681 & 0.12 & 322 & 680 & $\sim 350$ & $\begin{array}{l}\text { CA-l diffusion tubes left in; } \\
\text { filter unit changed. New } \\
\text { filter unit included silver } \\
\text { membrane filter, fiberglass } \\
\text { filter and charcoal absorber } \\
\text { for I. }\end{array}$ & Figs. 3.1-7. \\
\hline Св & Downstream & $1 / 17 / 73-5 / 19 / 73$ & 2688 & 0.23 & 585 & 300 & $\sim 130$ & $\begin{array}{l}\text { Four silver diffusion tubes, } \\
\text { diffusion tube, D, contained } \\
\text { flow constrictor, five-stage } \\
\text { particulate impactor, silver } \\
\text { membrane filter, fiberglass } \\
\text { filter, zeolite absorber, and } \\
\text { charcoal absorber. }\end{array}$ & Figs. $3.1-3,3.1-8$. \\
\hline $\mathrm{CC}$ & Upstream & $3 / 13 / 74-5 / 28 / 74$ & 1834 & 0.12 & 216 & 680 & $\sim 350$ & Same as for $\mathrm{CA}-2$. & \\
\hline$C D$ & Downstream & $3 / 13 / 74-5 / 28 / 74$ & 1834 & 0.17 & 319 & 300 & $\sim 130$ & Same as for СB. & \\
\hline
\end{tabular}




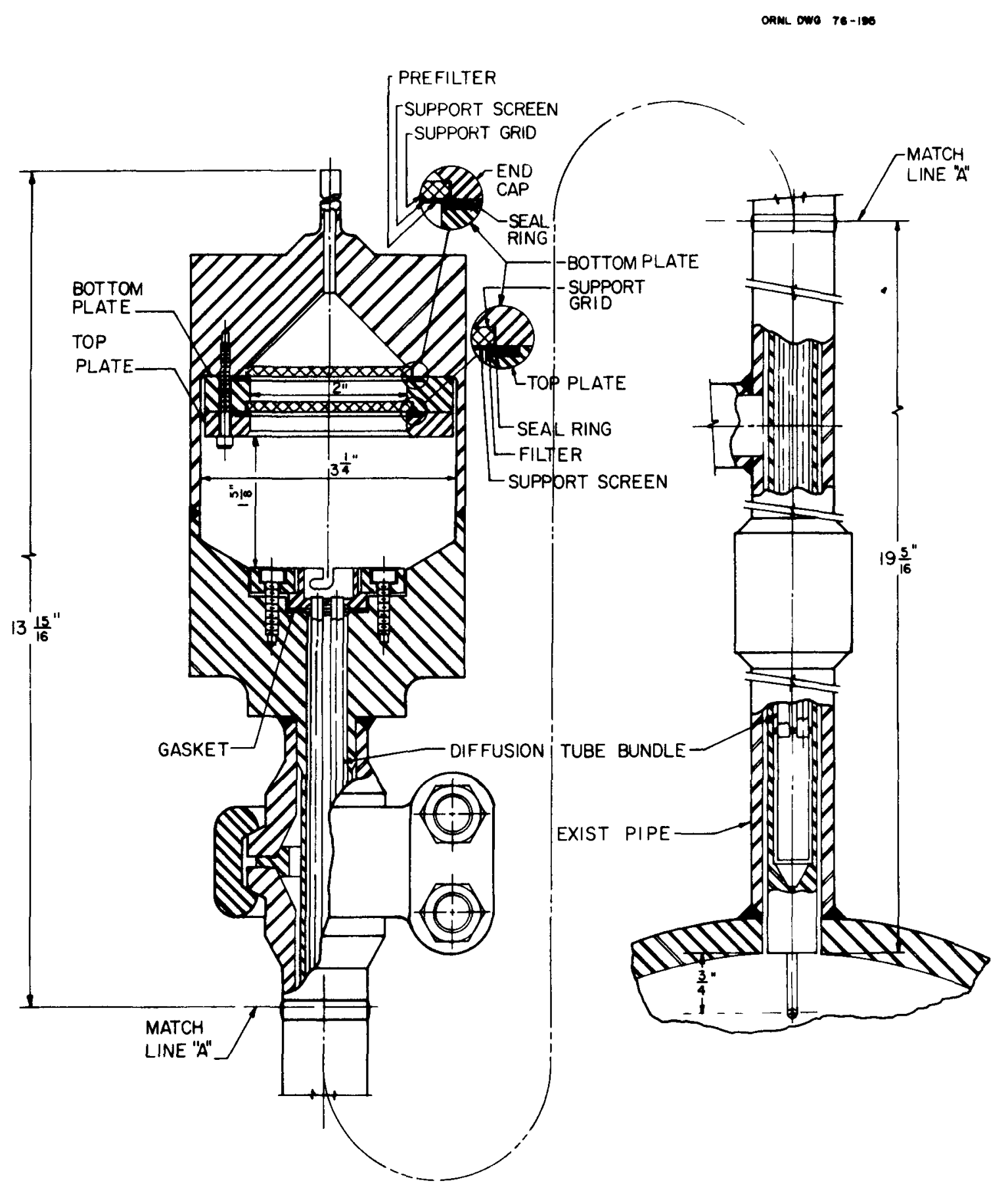

Fig. 3.1-2. Downstream sampler, Cl, assembly. 
ORNL DWG. $76-1249 R 1$

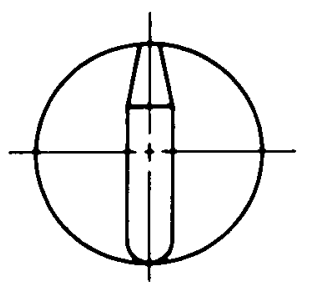

O.041 DIAM HOLE ENTIRE LENGTH

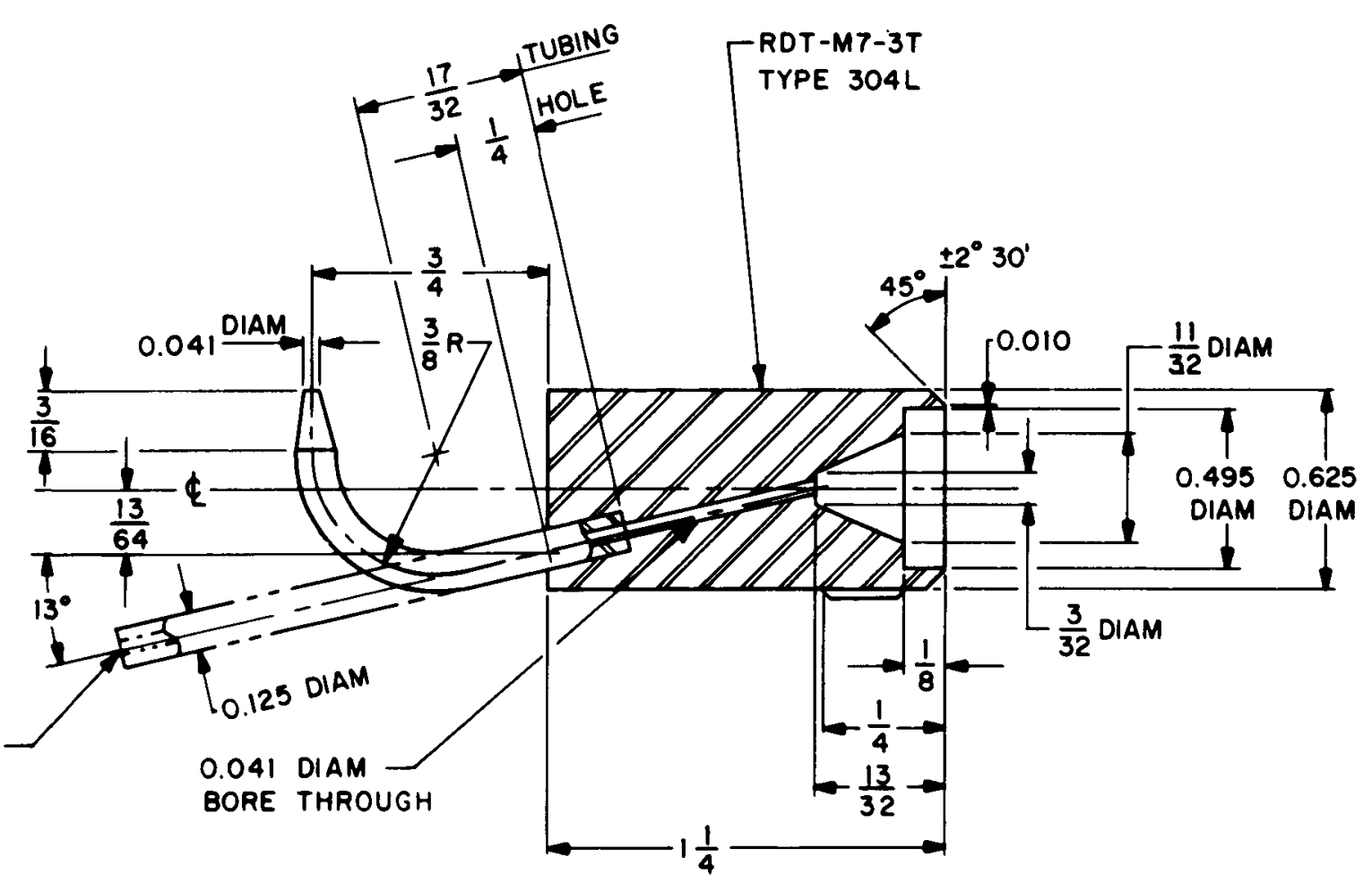
(dimensions in inches). 

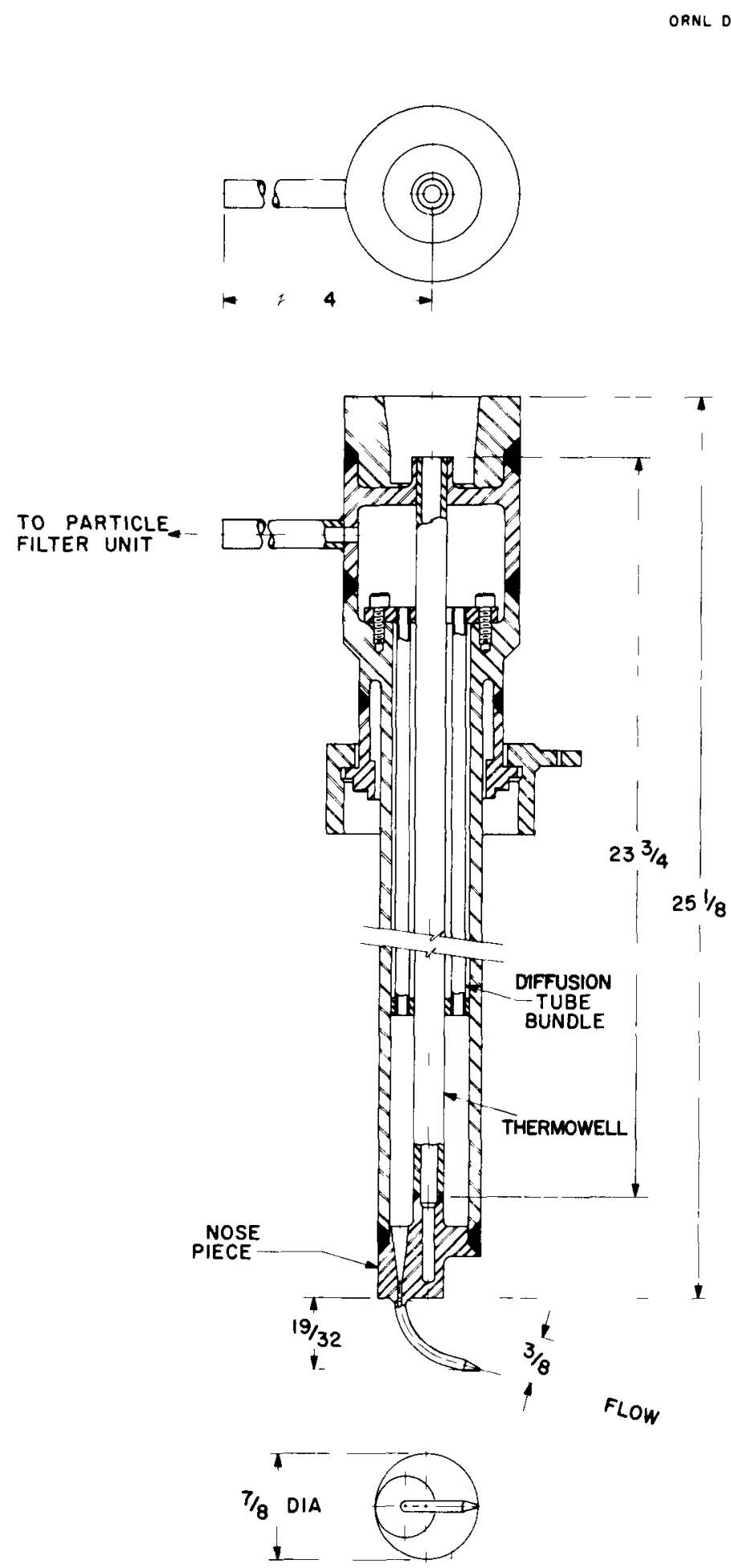

INSIDE WALI

He DUCT

Fig. 3.1-4. Upstream sampler, CA, assembly (dimensions in inches). 

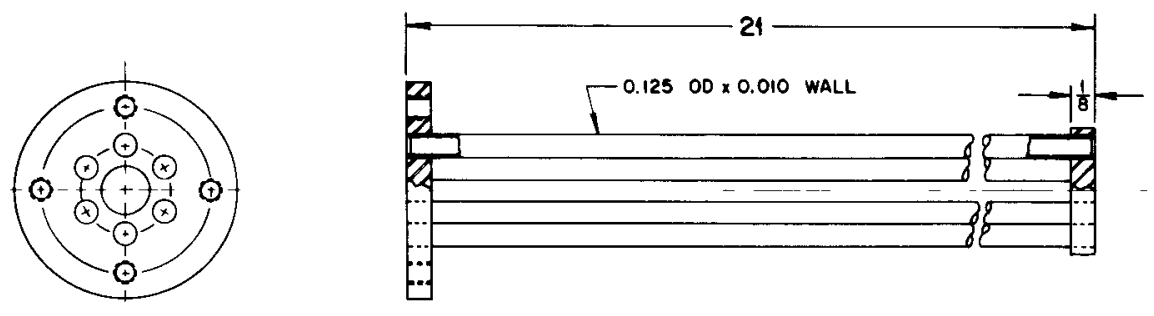

TUBE BUNDLE ASSEMBLY

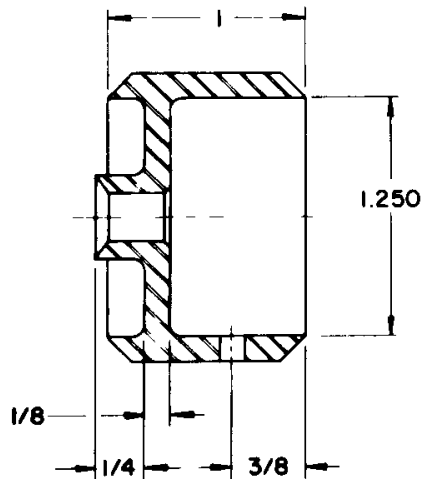

TOP END CAP

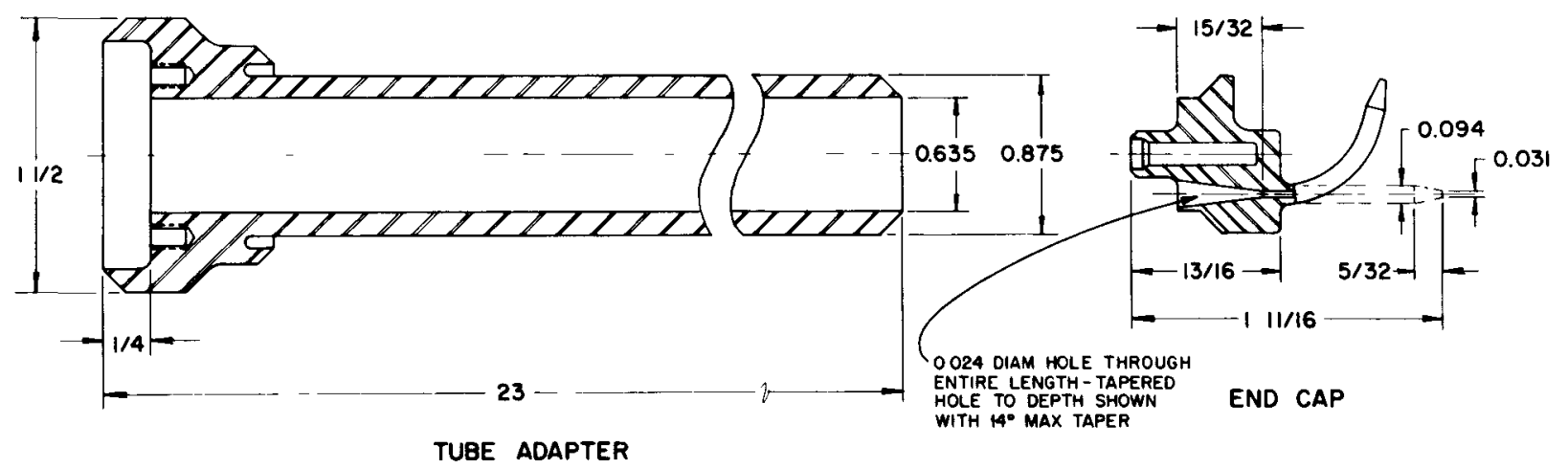

TUBE ADAPTER

Fig. 3.1-5. Upstream sampler, CA. Details of nozzle and diffusion tubes (dimensions in inches). 


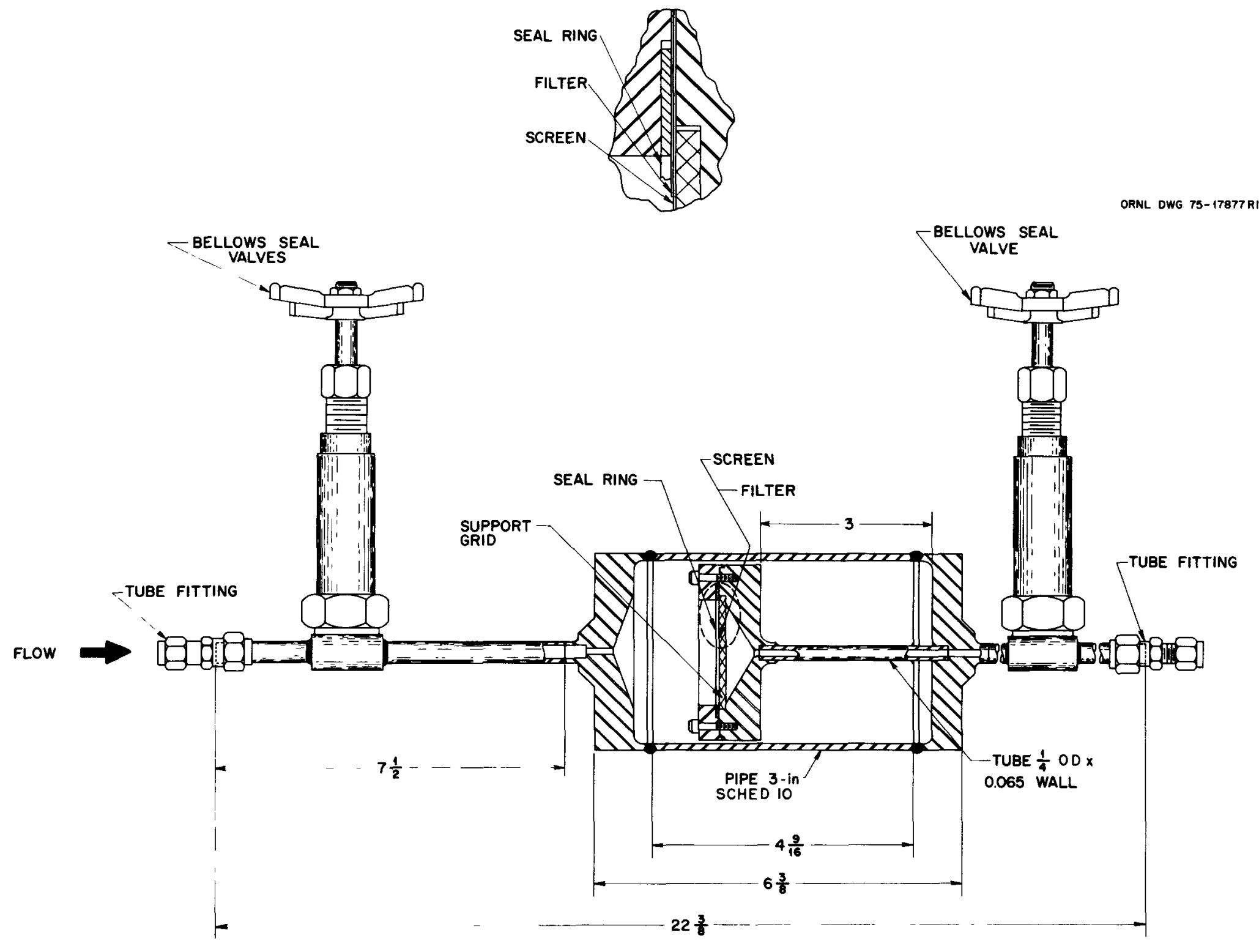

Fig. 3.1-6. Filter unit for sampler $\mathrm{CA}-1$ (dimensions in inches). 


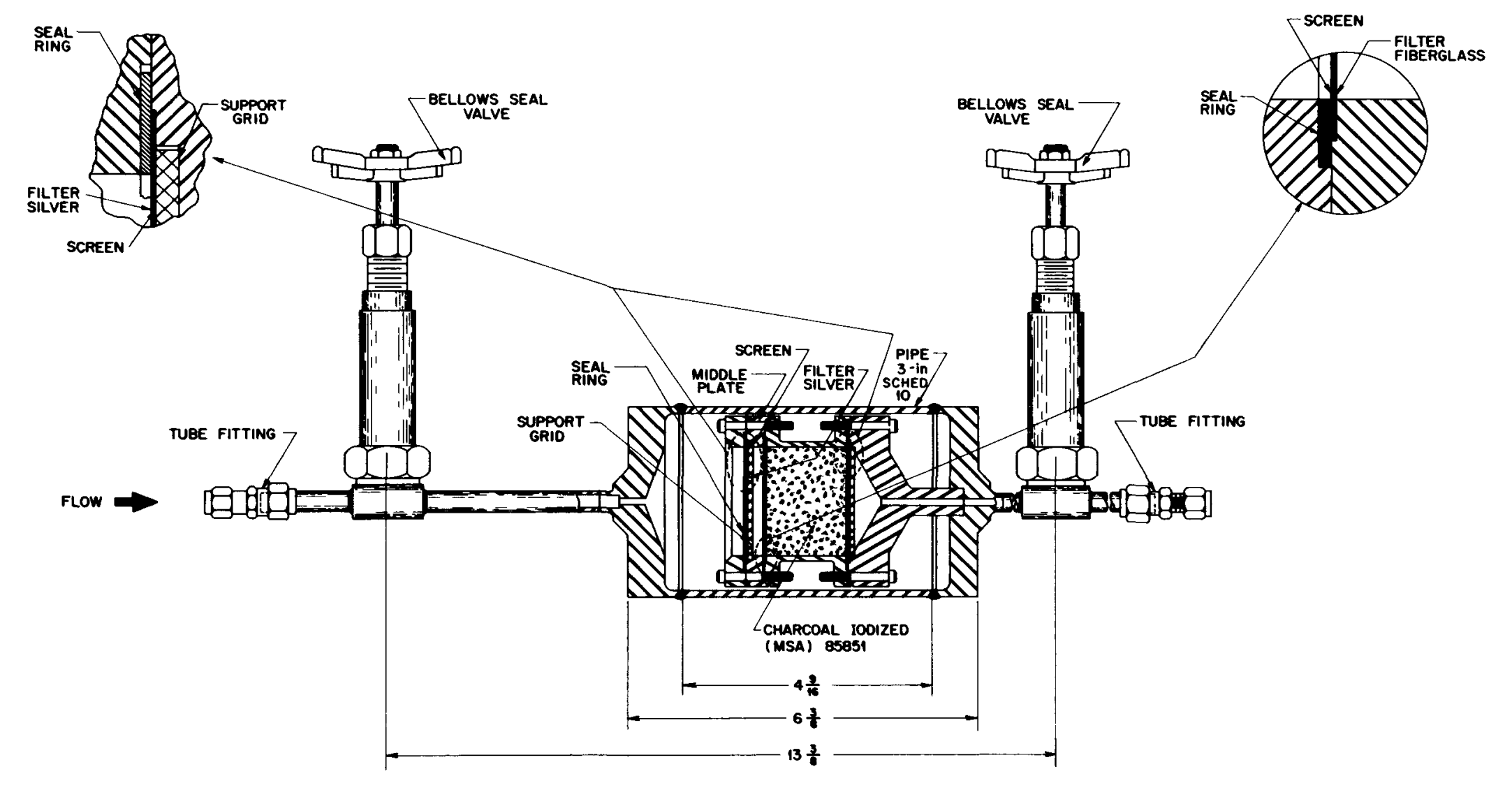

Fig. 3.1-7. Filter unit for sampler CA-2 (dimensions in inches). 
The connecting tube between the diffusion tube unit and the filter unit of the upstream sampler was removed and gamma-scanned after the operation of the CG sampler. The same tube was kept for the operation of the CA and CC samplers and was measured after the operation of the CC sampler. Thus, the amounts of long-lived radionuclides $\left({ }^{60} \mathrm{Co}, 134 \mathrm{Cs}\right.$, $137 \mathrm{Cs}$ ) measured in the tube in 1974 (see Table 3.2-5) were collected during previous operations of the CA and the CC samplers.

The most sophisticated of sampler assemblies used for the downstreamlocation experiments, designated as $\mathrm{CB}$ and $\mathrm{CD}$, is shown in Fig. 3.1-8. The unit contained a five-stage impactor for classification of particulates directly downstream from the diffusion tube assembly. Downstream from the impactor, the sample gas flowed through a silver membrane filter, an absolute filter composed of fiberglass, and then through a silver zeolite and charcoal absorber section.

The diffusion tubes for the $C B$ and $C D$ samplers consisted of a set of four silver tubes that had a 0.156-in. OD, a 0.010-in. wall and were $219 / 16$ in. Iong $(0.396 \mathrm{~cm} \times 0.0254 \mathrm{~cm} \times 54.8 \mathrm{~cm})$. One tube of this set (tube D) contained a flow constriction at its outlet consisting of a 1.9-cm-long cylindrical insert with a 0.012-in $(0.030-\mathrm{cm})$ hole. Therefore, very nearly the coolant flow through samplers $C B$ and $C D$ divided equally between tubes $\mathrm{A}, \mathrm{B}$, and $\mathrm{C}$.

The five-stage cascade impactor used in the $C B$ and $C D$ samplers had a design modeled after inertial impactors of proven operation, as described in the open literature. ${ }^{1}$ Critical parameters of the impactor stages are given in Table 3.1-2, and a photograph of the components is shown in Fig. 3.1-9. The impactor consisted of a series of five jets ranging from 6.52- to $0.41-\mathrm{mm}$ diameter, each impinging on a circular particulate collection disk located from 2.44 to $0.82 \mathrm{~mm}$ behind the orifice plate forming each jet. Three of the orifices, for stages 2, 3, and 4, are seen at the center of the three disks shown at bottom in Fig. 3.1-9. The orifice and plate for stage 1 are located within the inlet nozzle piece, shown at right. The stage-five collection plate is shown at left, indicating the method of attachment to the orifice. 


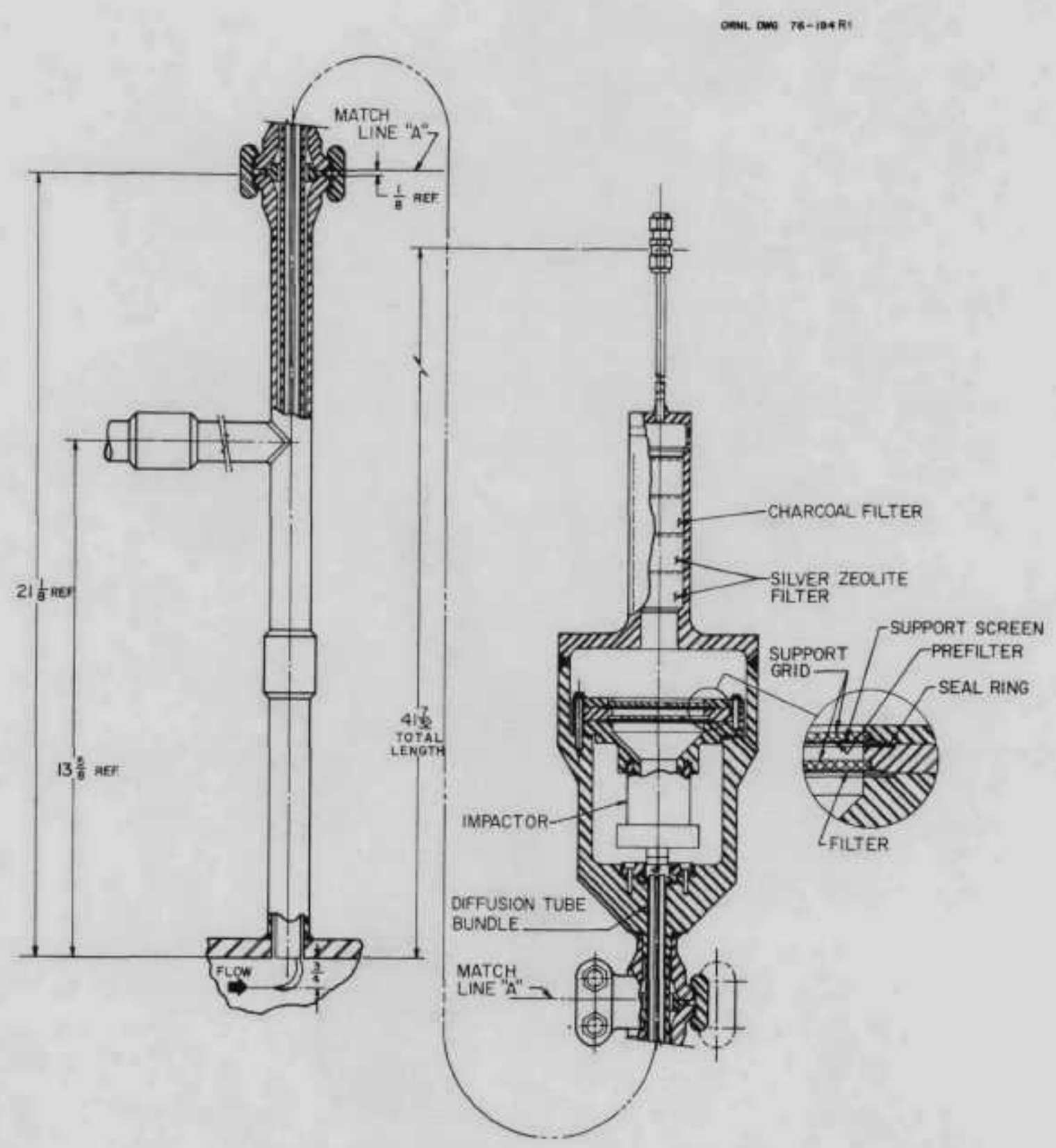

Fig. 3.1-8. Downstream sampler, $\mathrm{CB}$ and $\mathrm{CD}$ (dimensions in inches). 


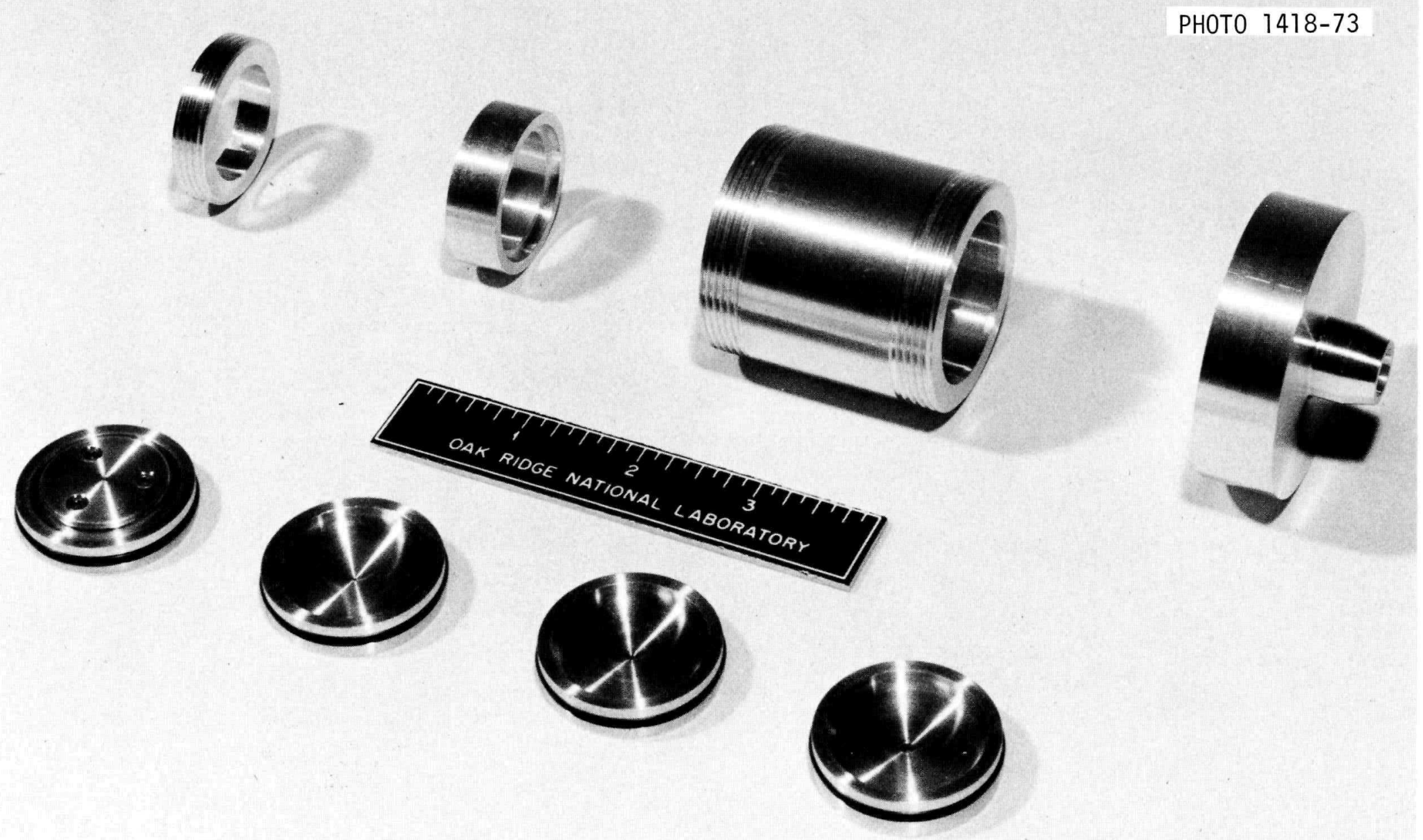

Fig. 3.1-9. Cascade impactor components. 
Table 3.1-2. Impactor stage characteristics, CA and CD sampler

\begin{tabular}{|c|c|c|c|c|c|}
\hline $\begin{array}{c}\text { Stage } \\
\text { No. }\end{array}$ & $\begin{array}{l}\text { Jet } \\
\text { diam } \\
(\mathrm{mm})\end{array}$ & $\begin{array}{l}\text { Distance } \\
\text { orifice } \\
\text { to } \\
\text { impactor } \\
\text { (mm) }\end{array}$ & $\begin{array}{c}\text { Jet } \\
\text { velocity } \\
(\mathrm{m} / \mathrm{sec})\end{array}$ & $\begin{array}{c}D_{50} \sqrt{P_{p} C} b \\
(g / c m)^{1 / 2} \times 10^{4}\end{array}$ & $\begin{array}{c}\text { Particle } \\
\text { cutoff } \\
\text { diam } \\
\text { range } \\
(\mu \mathrm{m})\end{array}$ \\
\hline 1 & 6.52 & 2.44 & 0.538 & 20.0 & $9.0-20$ \\
\hline 2 & 2.51 & 1.25 & 3.63 & 5.0 & $2.6-5.0$ \\
\hline 3 & 1.23 & 1.23 & 15.1 & 1.8 & $0.8-1.8$ \\
\hline 4 & 0.646 & 1.29 & 54.8 & 0.7 & $0.3-0.7$ \\
\hline 5 & 0.410 & 0.820 & 136.0 & 0.354 & $0.15-0.35$ \\
\hline
\end{tabular}

arom Lorenz taken from ref. 1.

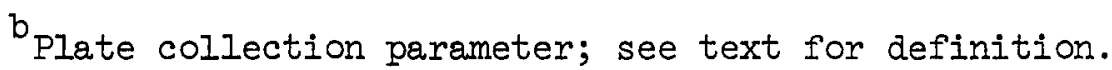

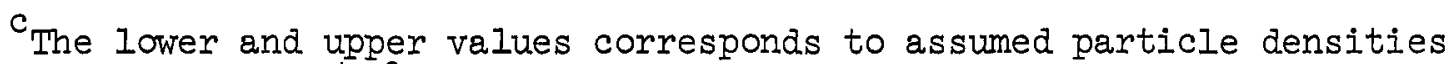
of 5 and $1.0 \mathrm{~g} / \mathrm{cm}^{3}$, respectively.

The impactor characteristics were based on a helium mass flow rate of $0.213 \mathrm{~kg} / \mathrm{hr}$ at a pressure of $23.8 \mathrm{~atm}(2.41 \mathrm{MPa})$ and a temperature of $79^{\circ} \mathrm{C}$. The collection characteristics of each stage are expressed in terms of the parameter, $D_{50} \sqrt{P_{p} C}(g / c m)^{1 / 2}$, indicated in Table 3.1-2, where $D_{50}$ is the particle size that is collected with $50 \%$ efficiency (also called the cutoff diameter), $P_{p}$ is the particle density, and $C$ is the Cunningham slip factor. Expression is made in this fashion because $P_{p}$ is not known, and the value of $C$ is dependent on $p_{p}$ to some extent. However, for practical purposes, particulate densities probably range from $\sim 1$ to $5 \mathrm{~g} / \mathrm{cm}^{3}$; the dust, being composed primarily of silica, alumina, graphite, iron oxide in various degrees of agglomeration, and $\mathrm{C}$, has a value of approximately unity for this density range. On this basis, the values for the particle cutoff diameter for each stage listed in Table 3.1-2 were estimated.

Further details regarding the sampler systems CA through CD may be obtained from the quality assurance report by Kolb. ${ }^{2}$ 


\subsection{Tabulated Results}

\subsubsection{Radionuclide inventories on sampler components}

The measured nuclide inventories for the various components of samplers $C G, C l, C A, C B, C C$, and $C D$ are summarized in Tables 3.2-I through $3 \cdot 2-6$.

It should be noted that the significance of the nozzle portion of the samplers was not fully recognized until late in the program, by which time the shorter lived radionuclides had decayed. The tabular data indicate that some nuclides strongly tended to deposit in the small nozzle portion of the assembly. For example, in most cases, $\sim 50$ to $75 \%$ of the total Cs observed in the diffusion tube, barrel, and nozzle was found in

the nozzle portion of the sampler. On the other hand, $110 \mathrm{~m} \mathrm{Ag}$ showed a smaller tendency to deposit in the nozzle, as seen from the Cl sampler results listed in Table $3.2-2$.

\subsubsection{Dust collected on impactor stages and filters}

Visual observation of $\mathrm{CB}$ and $\mathrm{CD}$ sampler impactor stages.

Figure 3.2-I illustrates the appearance of the impactor stages removed after sampler CB operation. Note the heavy deposits of an apparently sootlike material on each stage. These large deposits may be correlated with the existence of an oil leak into the primary system during sampler operation (see discussion p. 55). Impactor stages heavily loaded in this fashion are not expected to function as predicted by the design equations which assume smooth plate and no reentrainment effects. Figure 3.2-2 shows a view of the particulates taken near the edge of the deposit of each stage magnified 50X, and Fig. 3.2-3 illustrates the appearance of the heavy deposit on stage 1 at $16 \mathrm{x}$ magnification.

In contrast to the heavily loaded $C B$ stages, the $C D$ sampler impactor plates were fairly clean, as seen in Fig. 3.2-4, indicating significantly lower dust levels in the primary system during CD sampler operation.

The appearance of the silver membrane filters after $1834 \mathrm{hr}$ of operation in the CC (upstream from the steam generator) and CD (downstream) 
Table 3.2-1. Sumnary of activities on CG sampler (uCi, April 22, $1971^{\mathrm{a}}$ )

\begin{tabular}{|c|c|c|c|c|c|c|c|c|c|c|c|c|c|}
\hline Component & & ${ }^{51} \mathrm{Cr}$ & ${ }^{54} \mathrm{Mn}$ & $59_{\mathrm{Fe}}$ & ${ }^{60} \mathrm{Co}_{\mathrm{O}}$ & ${ }^{65} \mathrm{zn}$ & $131_{I}$ & $110 \mathrm{~m}$ & ${ }^{134} \mathrm{cs}$ & ${ }^{136} \mathrm{cs}$ & ${ }^{137} \mathrm{cs}$ & ${ }^{90} \mathrm{Sr}$ & ${ }^{137} \mathrm{Cs} /{ }^{134} \mathrm{Cs}$ \\
\hline \multirow[t]{2}{*}{ Diffusion tubes } & 0 & 0.030 & 0.011 & 0.036 & 0.008 & 0.026 & 0.0015 & 0.036 & 0.045 & $\mathrm{ND}^{\mathrm{b}}$ & 0.13 & $\sim 0.0062$ & 2.9 \\
\hline & 2 & 0.038 & 0.019 & 0.032 & 0.072 & 0.033 & 0.005 & 0.030 & 0.063 & ND & 0.17 & $\sim 0.011$ & 2.7 \\
\hline \multicolumn{2}{|l|}{$\begin{array}{l}\text { Connecting tube } \\
\text { between units }\end{array}$} & ND & 0.032 & ND & 0.0187 & ND & ID & $\mathbf{N D}$ & 0.274 & ND & 0.885 & c & 3.2 \\
\hline \multicolumn{2}{|l|}{ Filter } & ND & 0.062 & $\mathrm{ND}$ & 0.11 & 0.027 & ND & 0.038 & 0.45 & ND & 1.26 & & 2.8 \\
\hline \multicolumn{2}{|c|}{ Total dust $(\sim 50 \mathrm{mg})$} & $\mathbf{N D}$ & 0.214 & $\mathbf{N D}$ & 0.50 & 0.12 & ND & 0.11 & 1.36 & ND & 3.94 & & 2.9 \\
\hline \multicolumn{2}{|l|}{ Absorber } & ND & 0.0094 & $\mathrm{ND}$ & 0.012 & 0.0082 & ND & 0.0072 & 0.041 & ND & 0.098 & & 2.4 \\
\hline
\end{tabular}

${ }^{8}$ Only two diffusion tubes and filter unit were measured at ORNL; filter unit was not measured until after ${ }^{51} \mathrm{Cr},{ }^{59} \mathrm{Fe},{ }^{131_{I}}$, ${ }^{136} \mathrm{Cs}$, and ${ }^{89} \mathrm{Sr}$ had decayed.

bD indicates not detected.

$c_{\text {Blank indicates not measured. }}$ 
Table 3.2-2. Summary of activities on $\mathrm{Cl}$ sampler

(uCi, December 14, 1971)

\begin{tabular}{|c|c|c|c|c|c|c|c|c|c|c|c|c|}
\hline Component & $51_{C r}$ & ${ }^{54} \mathrm{Nm}$ & $59 \mathrm{Fe}$ & ${ }^{60} \mathrm{cc}$ & ${ }^{65} \mathrm{zn}$ & $110 m_{A B}$ & ${ }^{137}$ Cs & ${ }^{137} \mathrm{cs}$ & ${ }^{89} \mathrm{Sr}$ & ${ }^{90} \mathrm{Sr}$ & $235_{U}$ & ${ }^{137} \mathrm{cs} /{ }^{134} \mathrm{Cs}$ \\
\hline Inlet nozzle & $\mathrm{ND}^{\mathrm{a}}$ & 0.014 & 0.014 & 0.009 & 0.022 & 0.018 & 0.089 & 0.28 & & & & 3.1 \\
\hline Inlet manifold & b & & & & & & & & & & & \\
\hline $\begin{array}{ll}\text { Diffusion tubes } & 1 \\
2 \\
3\end{array}$ & $\begin{array}{l}\mathrm{ND} \\
\mathrm{ND} \\
\mathrm{ND}\end{array}$ & $\begin{array}{l}0.0040 \\
0.0062 \\
0.0052\end{array}$ & $\begin{array}{l}0.010 \\
0.017\end{array}$ & $\begin{array}{l}0.0042 \\
0.0062 \\
0.0065\end{array}$ & $\begin{array}{l}0.0013 \\
0.0021 \\
0.0021\end{array}$ & $\begin{array}{l}0.014 \\
0.017 \\
0.015\end{array}$ & $\begin{array}{l}0.014 \\
0.020 \\
0.021\end{array}$ & $\begin{array}{l}0.048 \\
0.071 \\
0.073\end{array}$ & 0.010 & 0.0019 & & $\begin{array}{l}3.4 \\
3.5 \\
3.5\end{array}$ \\
\hline $\begin{array}{l}\text { Total; jet, } \\
\text { diffusion tubes }\end{array}$ & & 0.0154 & & 0.0169 & 0.027 & 0.046 & 0.14 & 0.47 & & & & 3.4 \\
\hline Loose dust & $\mathbb{N D}$ & 0.0019 & ND & 0.0061 & $\mathbf{N D}$ & & 0.0046 & 0.015 & & & & $3 \cdot 3$ \\
\hline Filter 1 & 0.049 & 0.022 & 0.49 & 0.32 & 0.022 & 0.12 & 0.54 & 1.74 & 0.57 & 0.047 & & 3.2 \\
\hline Filter 2 & $\mathrm{ND}$ & 0.0022 & 0.002 & 0.005 & $\mathrm{ND}$ & $9.4 \times 10^{-4}$ & 0.0048 & 0.016 & & & & $3 \cdot 3$ \\
\hline
\end{tabular}

MD indicates not detected.

$b_{\text {Blank indicates not measured. }}$ 
Table 3.2-3. Summary of activities on $\mathrm{CA}$ sampler

( $\mu$ C1, May 18, 1973)

\begin{tabular}{|c|c|c|c|c|c|c|c|c|c|c|c|c|c|c|}
\hline Component & ${ }^{51} \mathrm{Cr}$ & $54 \mathrm{mn}$ & $59 \mathrm{Fe}$ & ${ }^{60} \mathrm{Co}$ & ${ }^{65} \mathrm{zn}$ & $110 \mathrm{~m}_{\mathrm{Ag}}$ & $231_{I}$ & ${ }^{134} \mathrm{Cs}$ & ${ }^{136} \mathrm{Cs}$ & ${ }^{137} \mathrm{Cs}$ & ${ }^{137} \mathrm{Cs} /{ }^{134} \mathrm{Cs}$ & ${ }^{140} \mathrm{Ba}$ & $89_{\mathrm{Sr}}$ & ${ }^{90} \mathrm{Sr}$ \\
\hline Inlet nozzle & $\mathbf{a}$ & & & & & & & 0.093 & & 0.098 & 1.1 & & & \\
\hline $\begin{array}{l}\text { Difrusion tubes: } \\
\text { Inlet mantfold }\end{array}$ & $3.7 \times 10^{-3}$ & $2.0 \times 10^{-3}$ & $3.4 \times 10^{-3}$ & $3.5 \times 10^{-3}$ & $0.5 \times 10^{-3}$ & $2.6 \times 10^{-3}$ & $3.1 \times 10^{-3}$ & 0.042 & $1.5 \times 10^{-3}$ & $\begin{array}{ll}3 & 0.048\end{array}$ & 1.1 & $N^{b}$ & & \\
\hline Tube A & 0.013 & $2.9 \times 10^{-3}$ & $5.4 \times 10^{-3}$ & $6.6 \times 10^{-3}$ & $2.9 \times 10^{-3}$ & $1.4 \times 10^{-3}$ & $6.7 \times 10^{-3}$ & 0.037 & $1.2 \times 10^{-3}$ & 30.076 & 2.0 & $5.5 \times 10^{-4}$ & & \\
\hline B & 0.0090 & $2.7 \times 10^{-3}$ & $4.8 \times 10^{-3}$ & $7.5 \times 10^{-3}$ & $2.5 \times 10^{-3}$ & $1.4 \times 10^{-3}$ & $8.2 \times 10^{-3}$ & 0.040 & $0.6 \times 10^{-3}$ & $\begin{array}{ll}3 & 0.082\end{array}$ & 2.0 & $4.8 \times 10^{-4}$ & & $4.1 \times 10^{-3}$ \\
\hline $\mathrm{c}$ & 0.011 & $2.7 \times 10^{-3}$ & $6.3 \times 10^{-3}$ & $6.3 \times 10^{-3}$ & $3.1 \times 10^{-3}$ & $1.1 \times 10^{-3}$ & $6.8 \times 10^{-3}$ & 0.042 & $1.3 \times 10^{-3}$ & $\begin{array}{ll}30.085 \\
\end{array}$ & 2.0 & $8.2 \times 10^{-4}$ & & \\
\hline D & 0.011 & $2.4 \times 10^{-3}$ & $5.5 \times 10^{-3}$ & $4.5 \times 10^{-3}$ & $3.0 \times 10^{-3}$ & $0.96 \times 10^{-3}$ & $5.0 \times 10^{-3}$ & 0.039 & $1.3 \times 10^{-3}$ & $\begin{array}{ll}30.076 \\
\end{array}$ & 1.9 & $5.4 \times 10^{-4}$ & & $2.0 \times 10^{-3}$ \\
\hline$E$ & 0.0092 & $2.2 \times 10^{-3}$ & $5.0 \times 10^{-3}$ & $4.3 \times 10^{-3}$ & $2.5 \times 10^{-3}$ & $0.90 \times 10^{-3}$ & $5.3 \times 10^{-3}$ & 0.036 & $1.3 \times 10^{-3}$ & $\begin{array}{ll}30.071 \\
0\end{array}$ & 2.0 & $5.4 \times 10^{-4}$ & & \\
\hline$F$ & 0.012 & $2.3 \times 10^{-3}$ & $6.4 \times 10^{-3}$ & $5.0 \times 10^{-3}$ & $2.8 \times 10^{-3}$ & $0.86 \times 10^{-3}$ & $6.5 \times 10^{-3}$ & 0.035 & $1.1 \times 10^{-3}$ & 30.072 & 2.1 & $5.3 \times 10^{-4}$ & & \\
\hline Outlet manifold & 0.019 & $3.5 \times 10^{-3}$ & 0.015 & 0.012 & $0.6 \times 10^{-3}$ & $0.70 \times 10^{-3}$ & $1.7 \times 10^{-3}$ & 0.010 & $0.4 \times 10^{-3}$ & $\begin{array}{ll}30.035 \\
\end{array}$ & 3.5 & ND & & \\
\hline $\begin{array}{l}\text { Total, diffusion tubes, } \\
\text { manifolds }\end{array}$ & 0.088 & 0.021 & 0.052 & 0.050 & 0.018 & $9.9 \times 10^{-3}$ & 0.043 & 0.37 & $9.1 \times 10^{-3}$ & 30.64 & 1.9 & & & \\
\hline \multicolumn{15}{|l|}{ Connecting tube } \\
\hline $\begin{array}{l}\text { Filters } \\
\text { Silver in } \mathrm{CA}-1^{\mathrm{C}}\end{array}$ & 0.052 & 0.029 & 0.12 & 0.054 & $1.5 \times 10^{-3}$ & 0.016 & $51.2 \times 10^{-3}$ & 0.090 & ND & 0.35 & 4.7 & $7.6 \times 10^{-3}$ & $6.4 \times 10^{-4}$ & 0.006 \\
\hline Silver in $C A-2$ & 0.074 & 0.094 & 0.20 & 0.19 & $7.4 \times 10^{-3}$ & 0.030 & $1.4 \times 10^{-3}$ & 0.25 & $6.4 \times 10^{-3}$ & $\begin{array}{ll}30.82 \\
0\end{array}$ & 3.3 & $\mathrm{ND}$ & & 0.186 \\
\hline Fiberglass in $\mathrm{CA}-2$ & $2.2 \times 10^{-3}$ & $3.9 \times 10^{-5}$ & $4.2 \times 10^{-5}$ & $8.6 \times 10^{-5}$ & $\mathrm{ND}$ & $8.0 \times 10^{-5}$ & $<3 \times 10^{-5}$ & ND & $\mathrm{ND}$ & $1.4 \times 10^{-4}$ & $-4 \quad \mathrm{ND}$ & ND & & \\
\hline Absorbers in $\mathrm{CA}-2$ & ND & ND & ND & $2.6 \times 10^{-4}$ & ND & ND & $5.2 \times 10^{-4}$ & $\mathrm{ND}$ & ND & $4.4 \times 10^{-3}$ & $0^{-3} \mathrm{ND}$ & $9.0 \times 10^{-3}$ & & \\
\hline Total in sampler ${ }^{d}$ & 0.16 & 0.11 & 0.25 & 0.24 & 0.025 & 0.040 & 0.045 & 0.62 & 0.015 & 1.46 & 2.6 & & & \\
\hline
\end{tabular}

Blank denotes that measurement was not made.

ND Indicates not detected.

${ }^{c}$ Filter No. 1 was removed $11 / 24 / 72$. Activities listed here were corrected to 5/19/73.

Excluding inlet nozzle, connecting tube, and CA-1 Ag-filter. 
Table 3.2-4. Summary of activities on CB sampler

$(\mu \mathrm{C1}$, May 18, 1973)

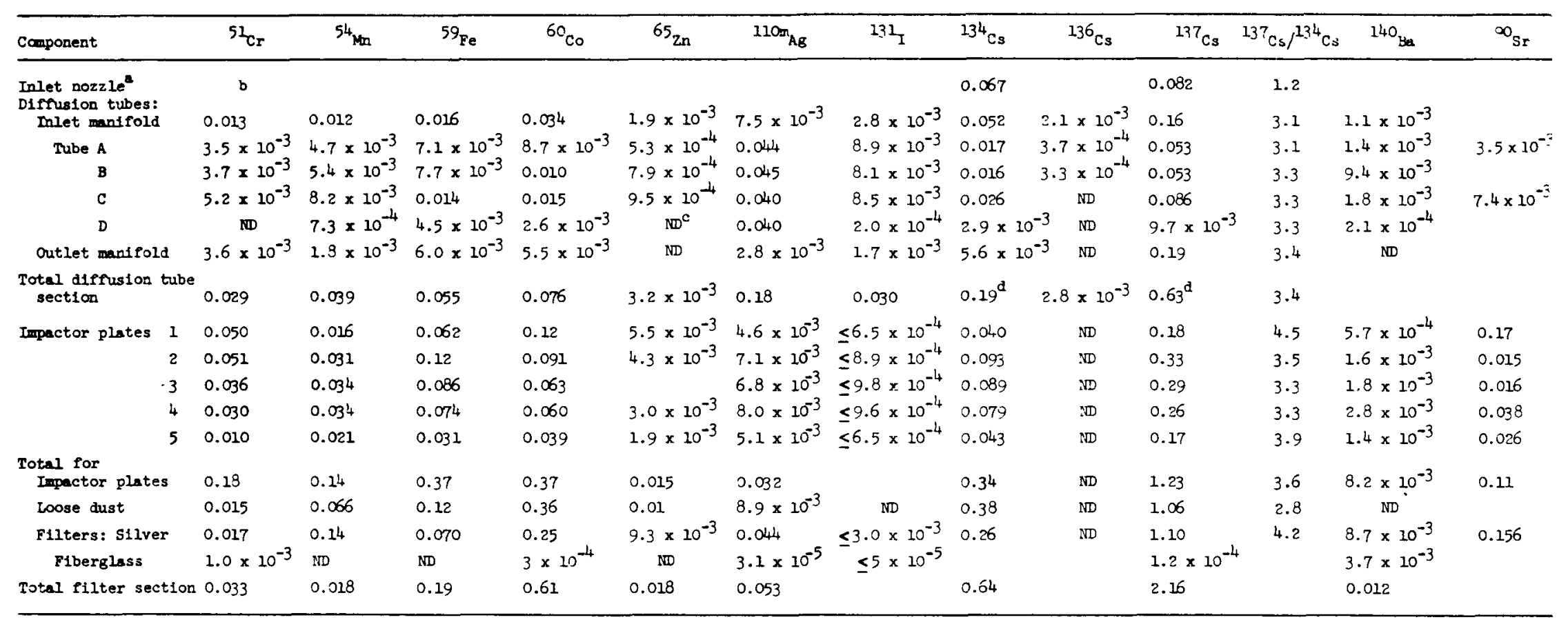

Inlet nozzle was not measured unt1l all ganma-emitters except ${ }^{134} \mathrm{Cs}$ and ${ }^{137} \mathrm{Cs}$ had decayed.

blank indicates not measured.

$c_{\mathrm{ND}}$ indicates not detected.

Lacludes inlet nozzle. 
Table 3.2-5. Summary of activities on CC sampler

( $\mu \mathrm{Ci}$, May 28, 1974)

\begin{tabular}{|c|c|c|c|c|c|c|c|c|c|c|c|c|}
\hline Component & CR5 I & $\pi \in R^{a}$ & MN 54 & 2ER & FE59 & XER & $c 060$ & XER & ZN 65 & ఇER & $A G 110$ & XER \\
\hline $\begin{array}{l}\text { Inlet nozzle } \\
\text { Diffusion tubes: } \\
\text { Inlet manifold }\end{array}$ & $1.70 \mathrm{E}-02$ & 22.0 & $8.30 E-04$ & 14.0 & $8.50 E-04$ & 13.0 & $1.60 E-03$ & $3 \cdot 7$ & $4.50 E-04$ & $*^{d}$ & $7.90 E-04$ & 15.0 \\
\hline Tube A & 2. $30 E-02$ & $14 \cdot 0$ & $3.50 E-04$ & 40.0 & $2.90 E-03$ & $7 \cdot 6$ & 2. $30 E-03$ & $4 \cdot 2$ & $1.05 E-03$ & 29.0 & $6 \cdot 19 E-04$ & 36.0 \\
\hline B & $1.20 E-02$ & 14.0 & $4.90 E-04$ & 40.0 & & & & & & & & \\
\hline C & $1.99 E-02$ & 10.0 & $4 \cdot 30 E-04$ & 29.0 & $2.40 E-03$ & 6.8 & $2 \cdot 10 E-03$ & $3 \cdot 4$ & $4 \cdot 68 E-04$ & $48 \cdot 0$ & $5 \cdot 70 E-04$ & 28.0 \\
\hline $\mathrm{D}$ & $7.60 E-03$ & $21 \cdot 0$ & $2 \cdot 58 E-04$ & 31.0 & $1.91 E-03$ & $7 \cdot 7$ & $2.03 E-03$ & $3 \cdot 4$ & 4. $32 E-04$ & $35 \cdot 0$ & $6.20 E-04$ & 23.0 \\
\hline$E$ & $1.82 E-02$ & $15 \cdot 0$ & $4.60 E-04$ & $22 \cdot 0$ & $2 \cdot 73 E-03$ & 6.8 & $2 \cdot 45 E-03$ & $3 \cdot 2$ & $5 \cdot 20 E-04$ & * & $4 \cdot 53 E-04$ & * \\
\hline$F$ & $1.56 E-02$ & 11.6 & $4 \cdot 56 E-04$ & 19.0 & 3. $23 E-03$ & 4.9 & $2.66 E-03$ & $2 \cdot 4$ & $5.04 E-04$ & $30 \cdot 0$ & $5.63 E-04$ & 27.0 \\
\hline $\begin{array}{l}\text { Outlet manifold } \\
\text { Total, diffusion tube } \\
\text { section }\end{array}$ & $\begin{array}{l}1.25 E-02 \\
1 \cdot 25 E-01\end{array}$ & 0.7 & $\begin{array}{l}2.05 E-04 \\
3.47 E-03\end{array}$ & 2.9 & $\begin{array}{l}1.11 E-03 \\
1.51 E-02\end{array}$ & $1 \cdot 3$ & $\begin{array}{l}9.30 E-04 \\
1.40 E-02\end{array}$ & 0.7 & $\begin{array}{l}3.95 E-05 \\
3.46 E-03\end{array}$ & 28.0 & $\begin{array}{l}4 \cdot 11 E-05 \\
3.65 E-03\end{array}$ & $\begin{array}{r}19.0 \\
0.0\end{array}$ \\
\hline Connecting tube & $8.30 E-01$ & $1 \cdot 6$ & $2 \cdot 10 E-03$ & $4 \cdot 8$ & $3.00 E-02$ & $5 \cdot 6$ & $2 \cdot 62 E-01$ & 0.8 & & & $5 \cdot 90 E-04$ & 17.0 \\
\hline $\begin{aligned} \text { Filters: } & \text { silver } \\
& \text { fiberglass }\end{aligned}$ & $2.91 E-02$ & 14.0 & $1.51 E-02$ & 1.9 & $4.09 E-02$ & 1.6 & $\begin{array}{l}5.27 E-02 \\
1.00 E-05\end{array}$ & $\begin{array}{r}0.8 \\
13.0\end{array}$ & $1.89 E-03$ & 27.0 & $1.15 E-02$ & 3.2 \\
\hline $\begin{array}{l}\text { Total, filter section } \\
\text { Adsorber }\end{array}$ & $8.59 E-01$ & & $1.72 E-02$ & & $7.09 E-02$ & & 3. $14 E-01$ & & $1.89 E-03$ & & $1.20 E-02$ & 0.0 \\
\hline Total in sampler & $9.84 E-01$ & & $2.06 E-02$ & & $8.60 E-02$ & & $3 \cdot 28 E-01$ & & 5. $35 E-03$ & & $1.57 E-02$ & 0.0 \\
\hline
\end{tabular}


Table 3.2-5. Continued

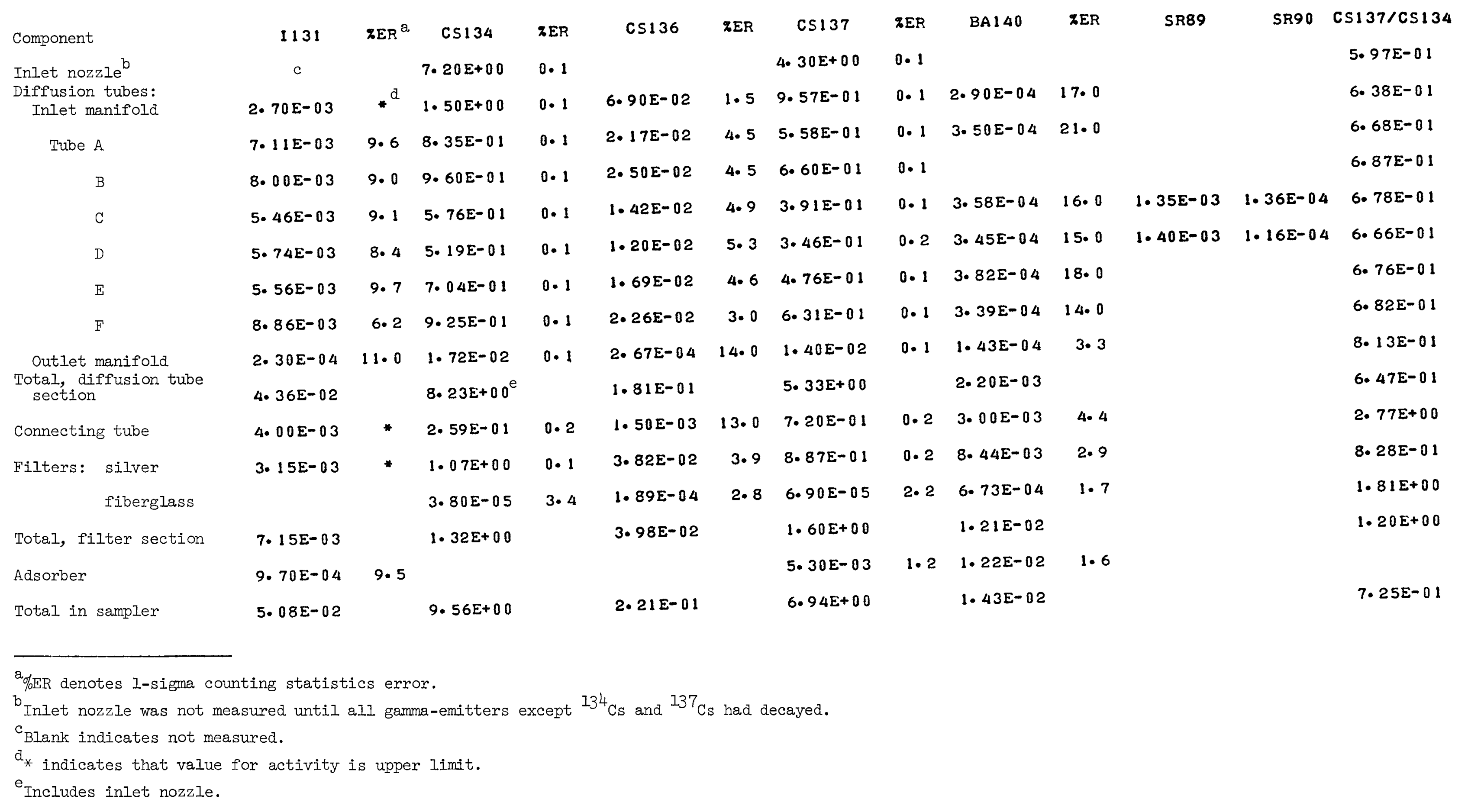


Table 3.2-6. Summary of activities on CD sampler ( $\mu \mathrm{Ci}$, May 28, 1974)

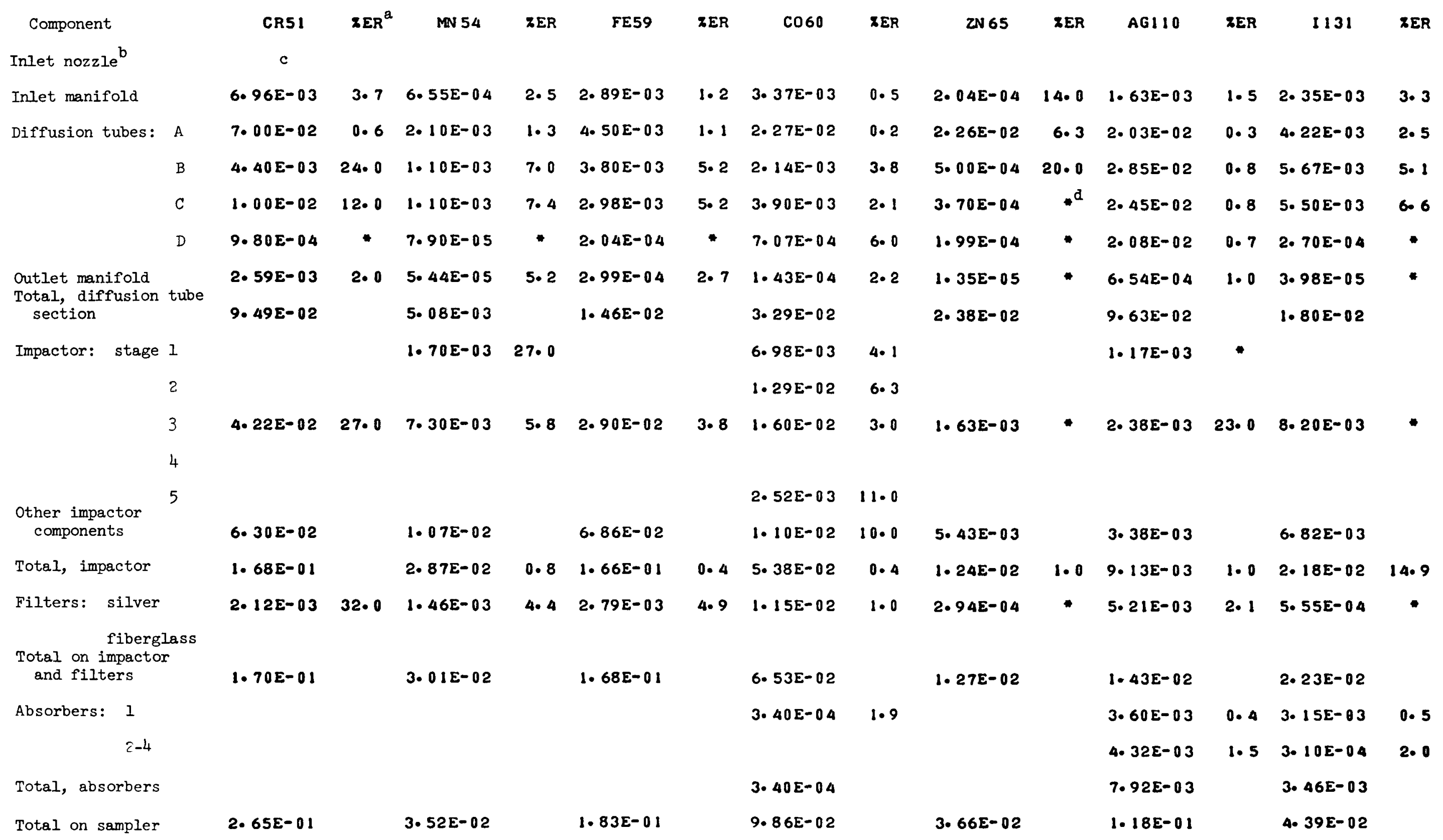


Table 3.2-6. Continued

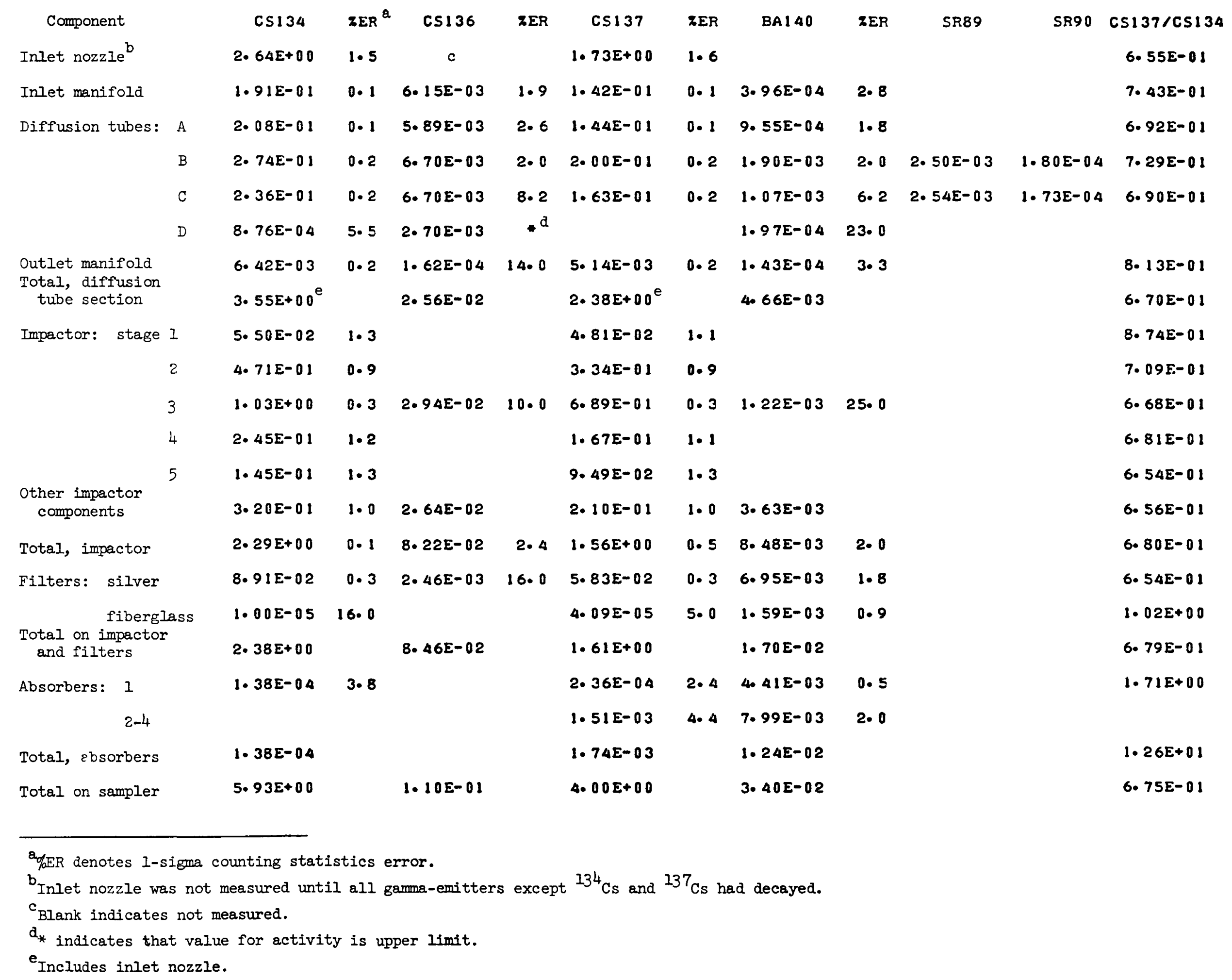


PHOTO $1275-73$

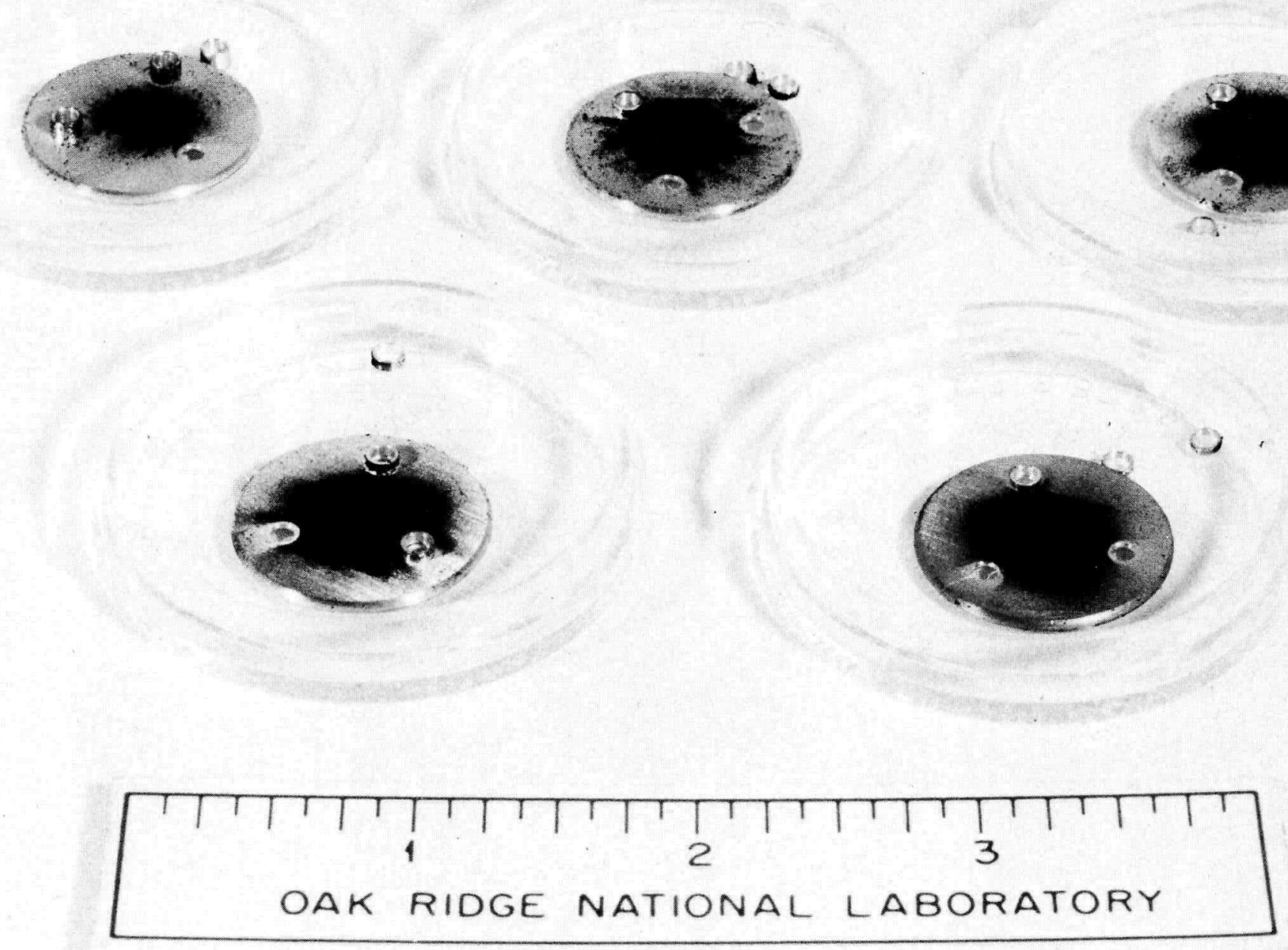

Fig. 3.2-1. Sampler CB impactor stages. Stages 1 and 2, bottom left to right; stages 3 to 5, top left to right. 


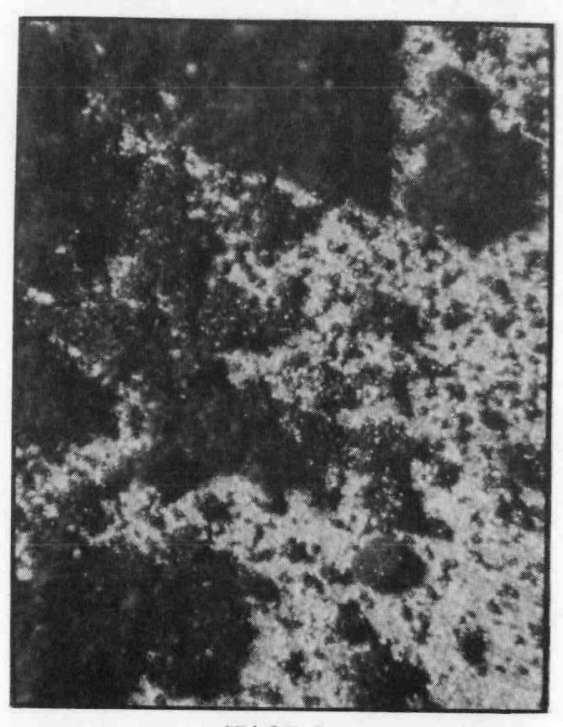

STAGE 1

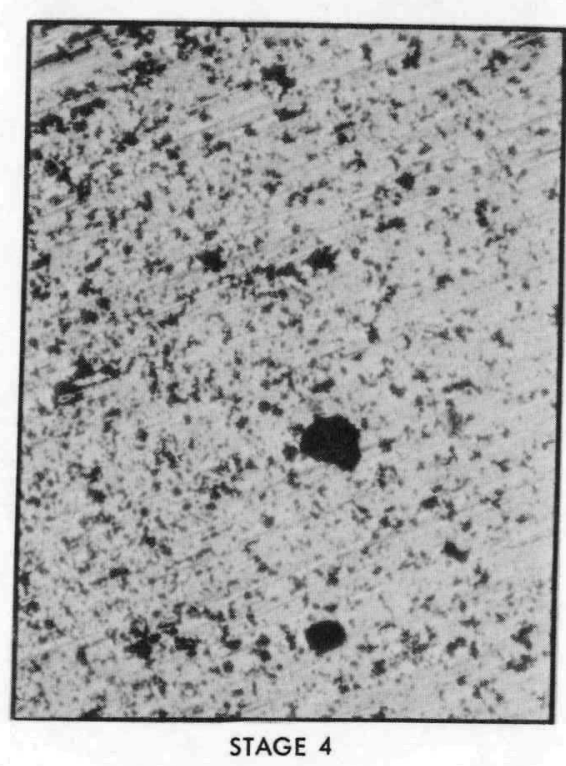

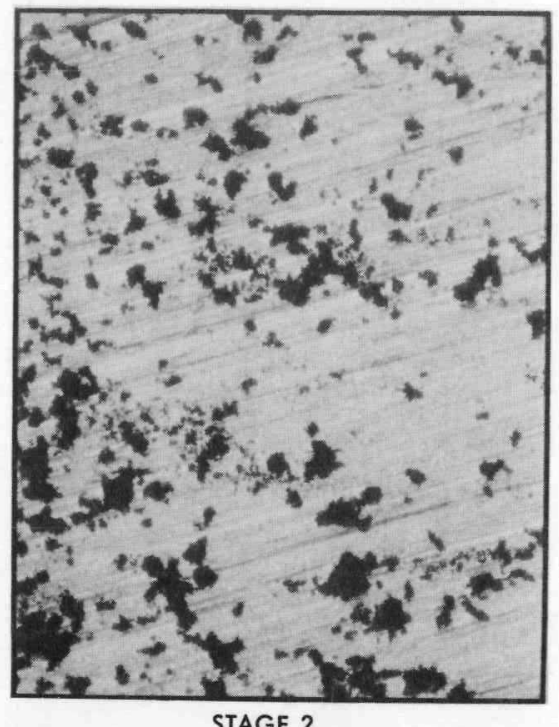

STAGE 2

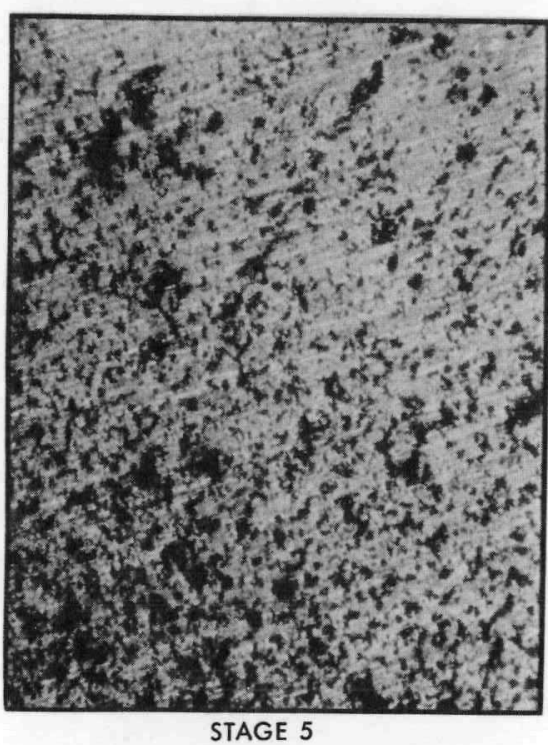

$\mathrm{Y}-120226$

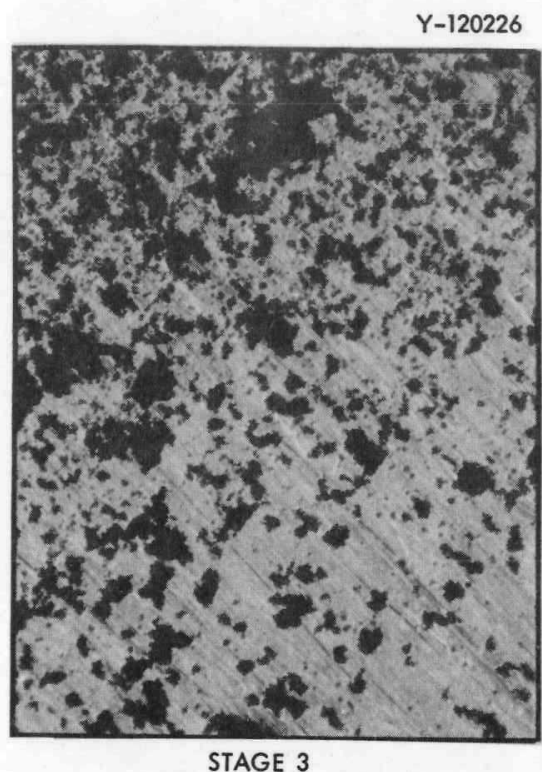

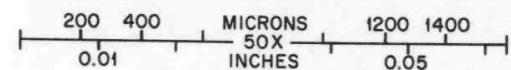
CASCADE IMPACTOR STAGES MAY 1973
PEACH BOTTOM DOWNSTREAM SAMPLER

Fig. 3.2-2. Impactor stages of $\mathrm{CB}$ sampler. Magnification $\sim 50 \mathrm{X}$. 


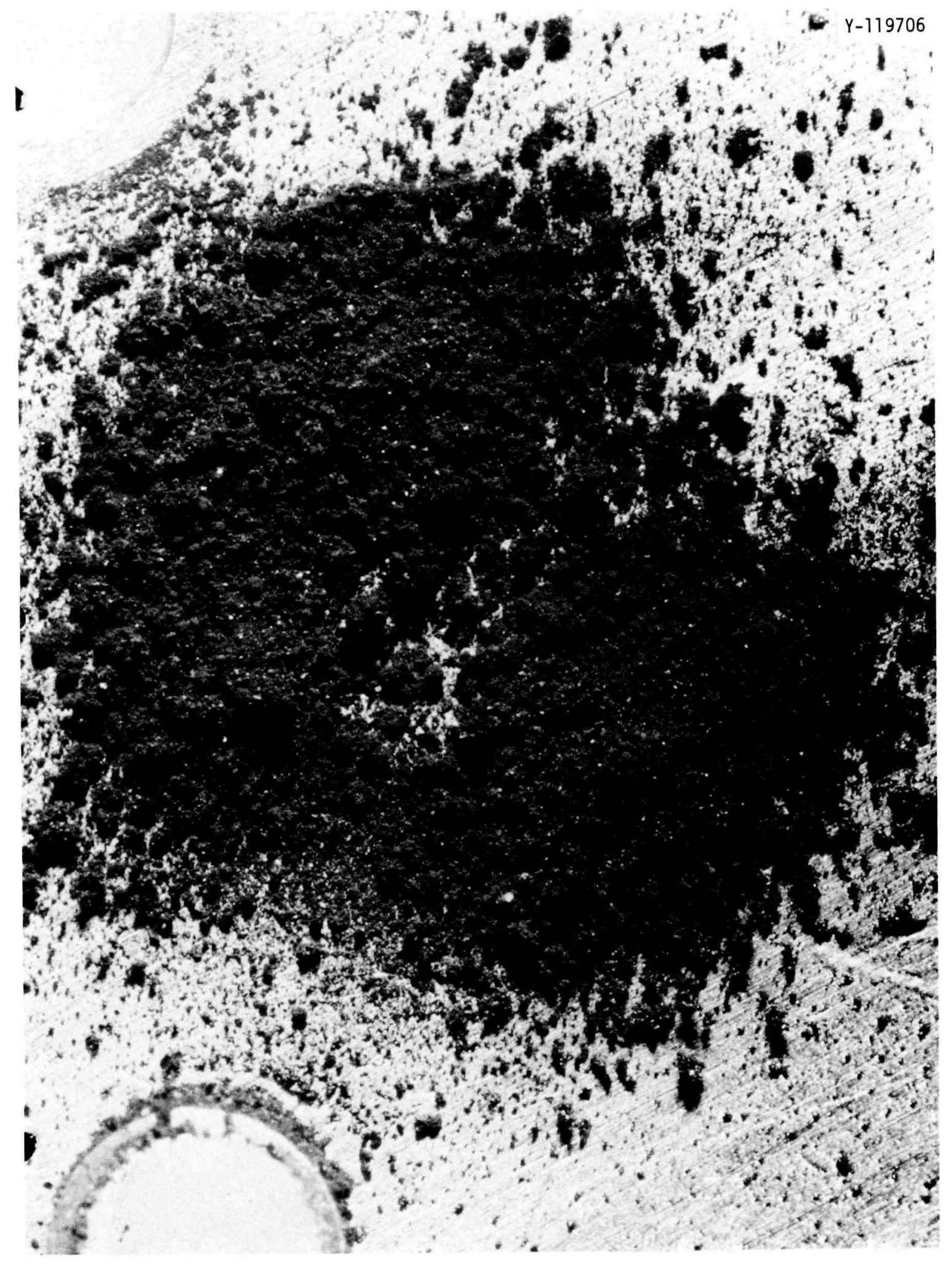

Fig. 3.2-3. View of stage-1, CB sampler. Magnification 16X. 


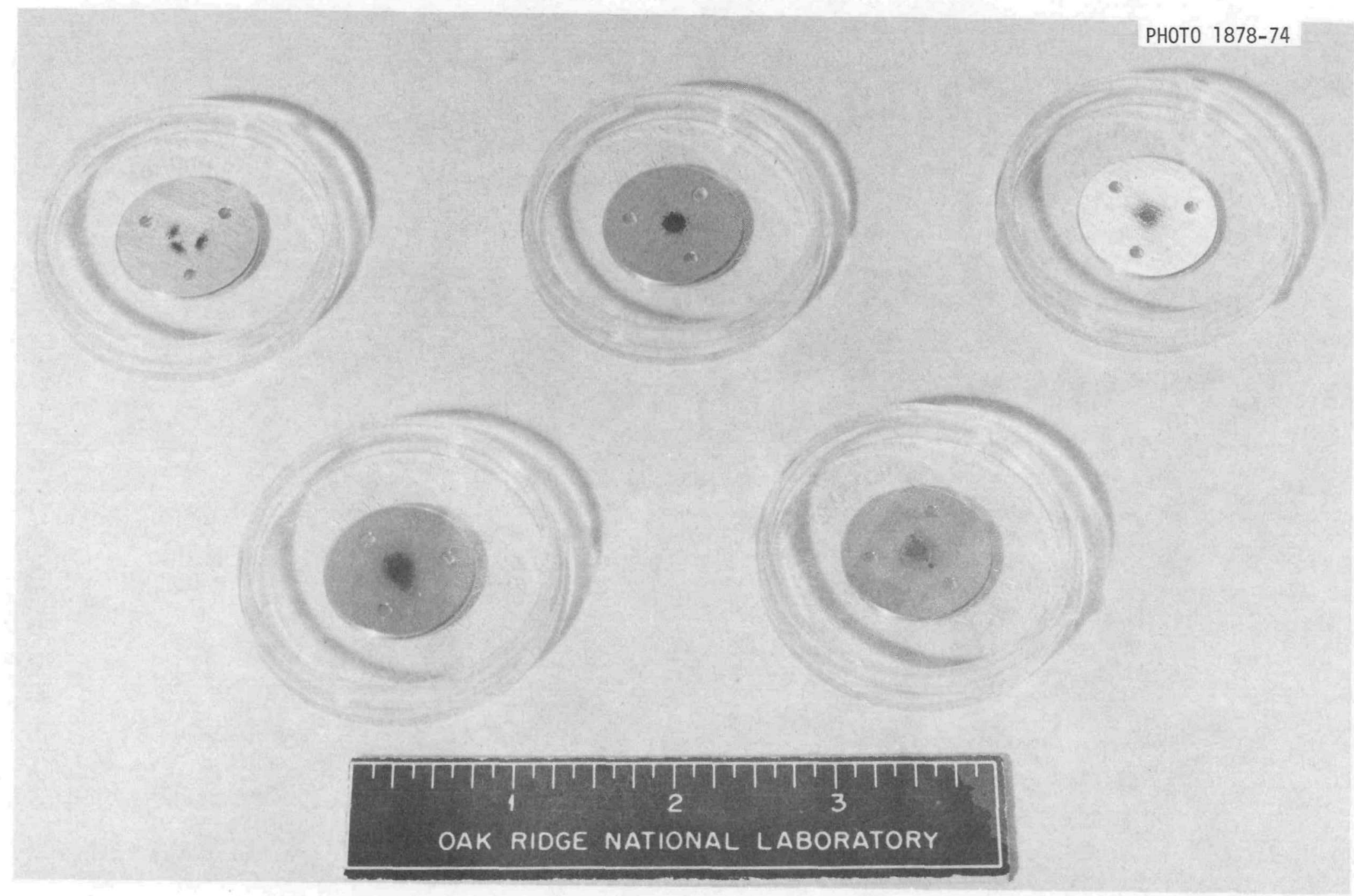

Fig. 3.2-4. Sampler CD impactor stages. Stages 1, 2, and 3 left to right; 4 and 5 below, left to right. 
coolant sanplers are shown et left and right, respectively, in Fig. 3.2-5. Note that no visible deposit was found on the dowstream filter element, whereas the upstream filter contains an obvious deposit. The visual appearance of the ailver membrane filters in samplers $\mathrm{Cl}, \mathrm{CA}-1, \mathrm{CA}-2$, and $\mathrm{CB}$, as well as the cellulose filter in CG, indicated heavier particulate deposits than indicated for the CC element. (Quantities of dust collected on each filter are given in Table 3.2-7.) The fiberglass obsolute filters behind each silver nembrane filter were, in all cases, devoid of deposits.

Table 3.2-7. Farticulates collected by coolant samplers

\begin{tabular}{|c|c|c|c|}
\hline \multirow[b]{2}{*}{ stage } & \multicolumn{2}{|c|}{ CB sampler } & \multirow{2}{*}{$\begin{array}{c}\text { CD sampler } \\
\text { Particulate } \\
\text { mess } \\
\text { (mg) }\end{array}$} \\
\hline & $\begin{array}{l}\text { Farticulate } \\
\text { mass } \\
\text { (m言) }\end{array}$ & $\begin{array}{l}235_{U} \\
(p+q)\end{array}$ & \\
\hline 1 & 5.62 & 2.35 & $<0.1$ \\
\hline 2 & 6.38 & 2.51 & $<0.2$ \\
\hline 3 & 4.37 & 1.58 & $<0.1$ \\
\hline 4 & 5.67 & 1.42 & $<0.1$ \\
\hline 5 & 3.85 & 1.38 & $<0.2$ \\
\hline \multirow[t]{2}{*}{ Coolant sampler } & & \multicolumn{2}{|c|}{$\begin{array}{c}\text { Mass of particulate collected on } \\
\text { filters }\end{array}$} \\
\hline & & (m官) & (me dust/foc coolant) \\
\hline $\mathrm{CG}$ & & $50 \pm \sim 1$ & 0.16 \\
\hline cl & & 24.0 & 0.041 \\
\hline$C A-1$ & & 8.0 & 0.039 \\
\hline$C A-2$ & & 22.5 & 0.070 \\
\hline $\mathrm{CB}$ & & 25.0 & $0.087^{\circ}$ \\
\hline $\mathrm{CO}$ & & 6.3 & 0.029 \\
\hline $\mathrm{D}$ & & Fo dust visible & $<0.002^{b}$ \\
\hline
\end{tabular}

a Collected on the cellulose filter in sampler co and the silver menbrane filters in samplers CA to CD. Ho particulates were observed on the absolute, fiberglass filters backing up the silver filters in samplers $\mathrm{CA}-2, \mathrm{CB}, \mathrm{CC}$, and $\mathrm{CD}$.

${ }^{b}$ Includes dust on impactor plates. 

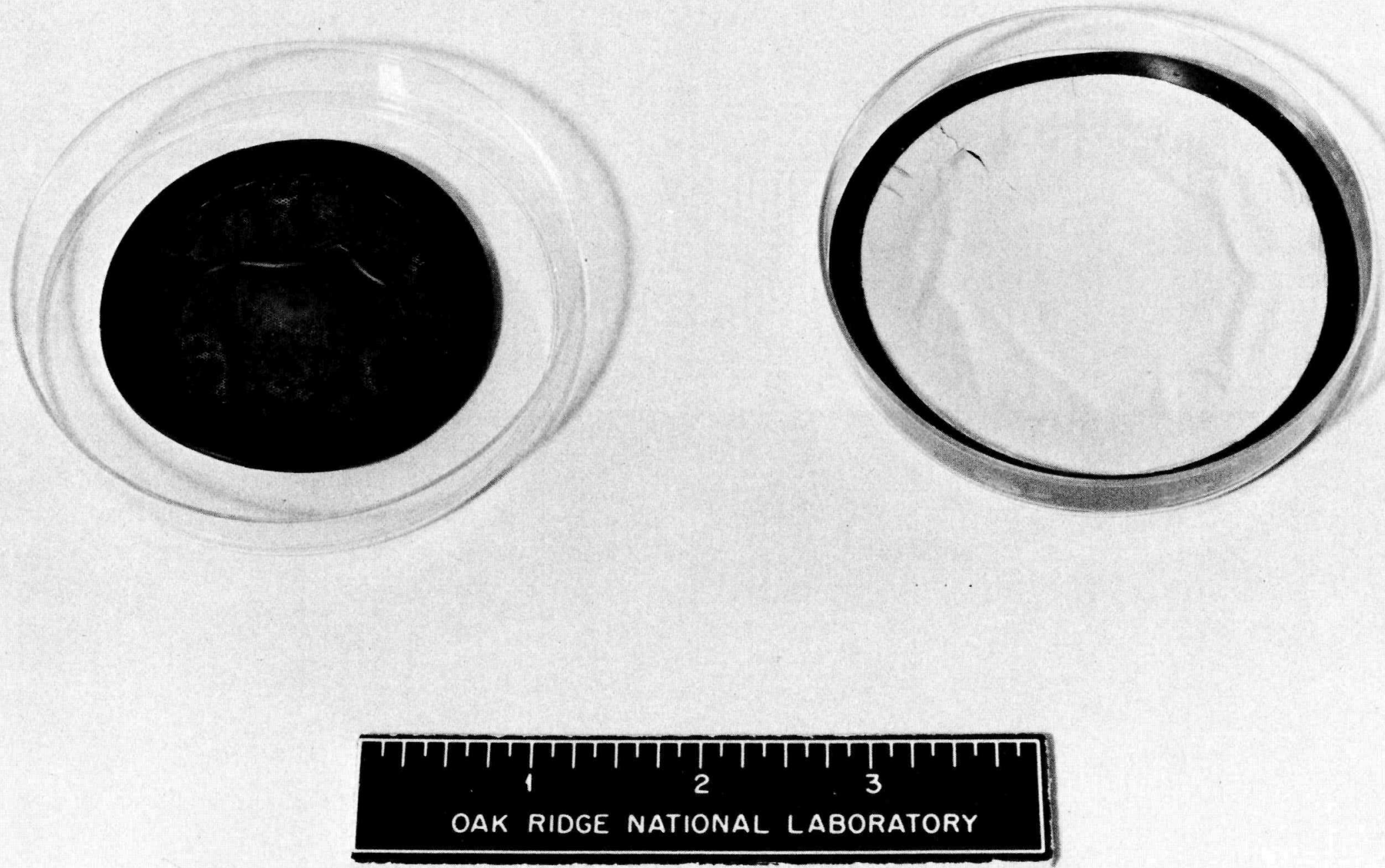

Fig. 3.2-5. Appearance of silver membrane filter after $1834 \mathrm{hr}$ of operation. Left: upstream, CC sampler; right: downstream, CD sampler. 
Lubricating oil leakage into the primary circuit. During the operation of Core 2 until Dec. 1972, occasional high levels ("spikes") of methane were observed in the primary coolant. ${ }^{3}$ Generally, the methane spikes, which were traced to oil inleakage from the purified helium compressor and poor efficiency of the oil removal system downstream from the compressor, were of short duration and did not exceed technical specifications for hydrocarbons in the coolant. However, in the fall of 1972, increased methane levels made it necessary to operate the reactor at reduced power levels to satisify technical specification requirements. ${ }^{3}$

In Dec. 1972, modifications were made to the "oil separator" downstream from the purified helium compressor, and the charcoal in the oil removal filter was replaced. ${ }^{4}$ These changes, along with modifications to the oil drain lines that had previously been installed on the purified helium return piping, resulted in a very significant decrease in the coolant hydrocarbon levels, and only a few small increased levels of methane were thereafter observed (e.g., higher than normal methane levels were observed for three days during a reactor startup on June 9, 1973).

The inleakages of lubricating oil undoubtedly affected primary system dust levels significantly, as noted by the coolant-sampler dust collections on filters and impactor stages (these data are summarized in Table 3.2-7) and the levels collected in the cyclone separators (see Sect. 5). The observation that the CB-sampler impactor stages collected significantly more particulates than the CD sampler (compare Figs. 3.2-1 and $3.2-4$ ), despite the fact that its operating period began directly following the leak-correcting measures, indicates that particulates generated by the oil leak persisted in the primary system beyond the date of leak termination.

Quantities of dust collected by the coolant samplers. Table 3.2-7 lists the amounts of particulate material collected on the $C B$ and $C D$ impactor stages and on the filter elements in each sampler.

Composition of dust on $\mathrm{CB}$ and $\mathrm{CD}$ sampler impactor stages. ${ }^{5}$ Attempts were made to identify the particulate material collected on the impactor stages by performing the following analyses: (1) x-ray induced $x$-ray 
fluorescent (XRI-XRF) spectra were acquired from sections of each stage; (2) scanning electron micrographs (SEM) were also obtained from various locations, and the induced $x$-ray fluorescence (SEM-XRF) spectra were analyzed; (3) x-ray diffraction patterns were obtained to determine molecular species.

Procedure 1 yielded an accurate determination of elements above vanadium on a macroscopic field of $\sim 3$ by $3 \mathrm{~mm}$. Procedure 2 enabled the inspection of minute volumes, down to $1 \mathrm{\mu m}^{3}$, in which the detection of elements above magnesium in the periodic table present in amounts exceeding 0.1 to $1 \%$ could be accomplished. The subject material was subsequently identified by collating the information gathered from the three procedures. One loophole in this scheme, however, is that amorphous carbon cannot be detected by any of the three procedures, although graphite can be detected by $x$-ray diffraction.

Figure 3.2-6 presents three views of a 0.1 by $0.1 \mathrm{~mm}$ field of particles from stages 2 of the $C B$ sampler. The top left view, at 1200X magnification, is a micrograph taken from the scanning electron image showing particles generally in the range of 1 to $8 \mu \mathrm{m}$. This compares reasonably well with the predicted performance of the impactor given on Table 3.1-2, where the range of sizes on stage 2 from 2.6 to $9.0 \mu \mathrm{m}$ is indicated for particles with a density of $\sim 5.0 \mathrm{~g} / \mathrm{cm}^{3}$. In Fig. $3.2-6$, the top right and bottom views are the so-called iron and silicon maps obtained with the SEM method by filtering the fluoresced $x$-ray at the desired wavelength. The maps display the distribution of iron and silicon in the field corresponding to the micrograph shown at top left. These illustrate an interesting feature generally observed for all the coolant-sampler dust specimens. There is a strong correlation between the locations of iron and silicon in the dust sample, which indicates some chemical combination between the two elements in the dust particles. However, examination of these particles by $\mathrm{x}$-ray diffraction failed to reveal any lines. It is therefore believed that silicon is present as an amorphous compound like glass, which would not yield diffraction lines, and in chemical combination with iron. 


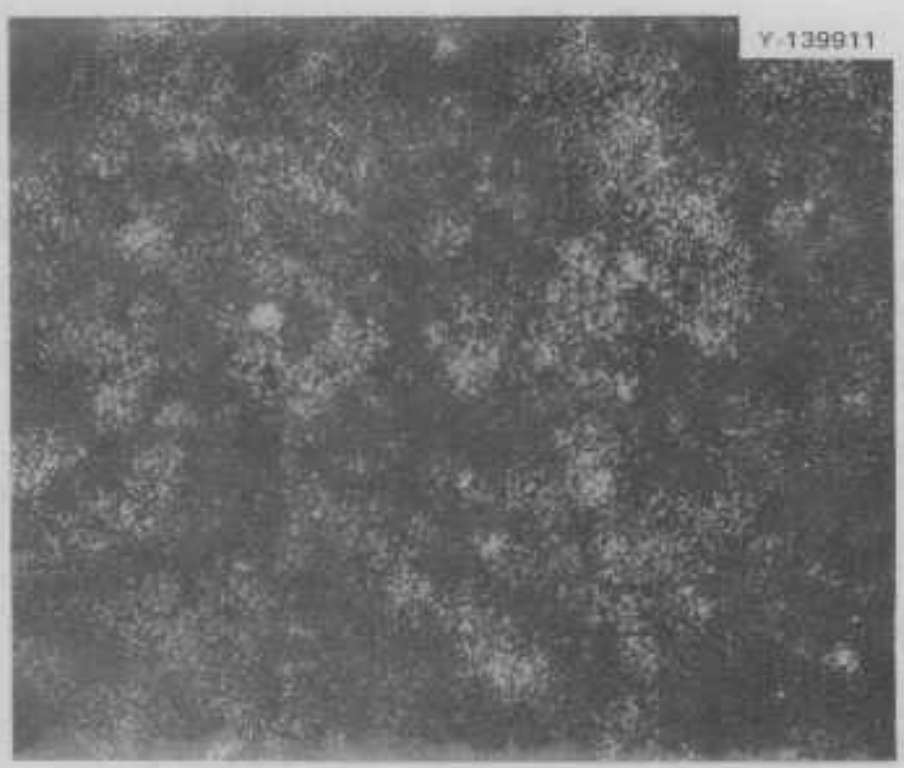

$1200 x$

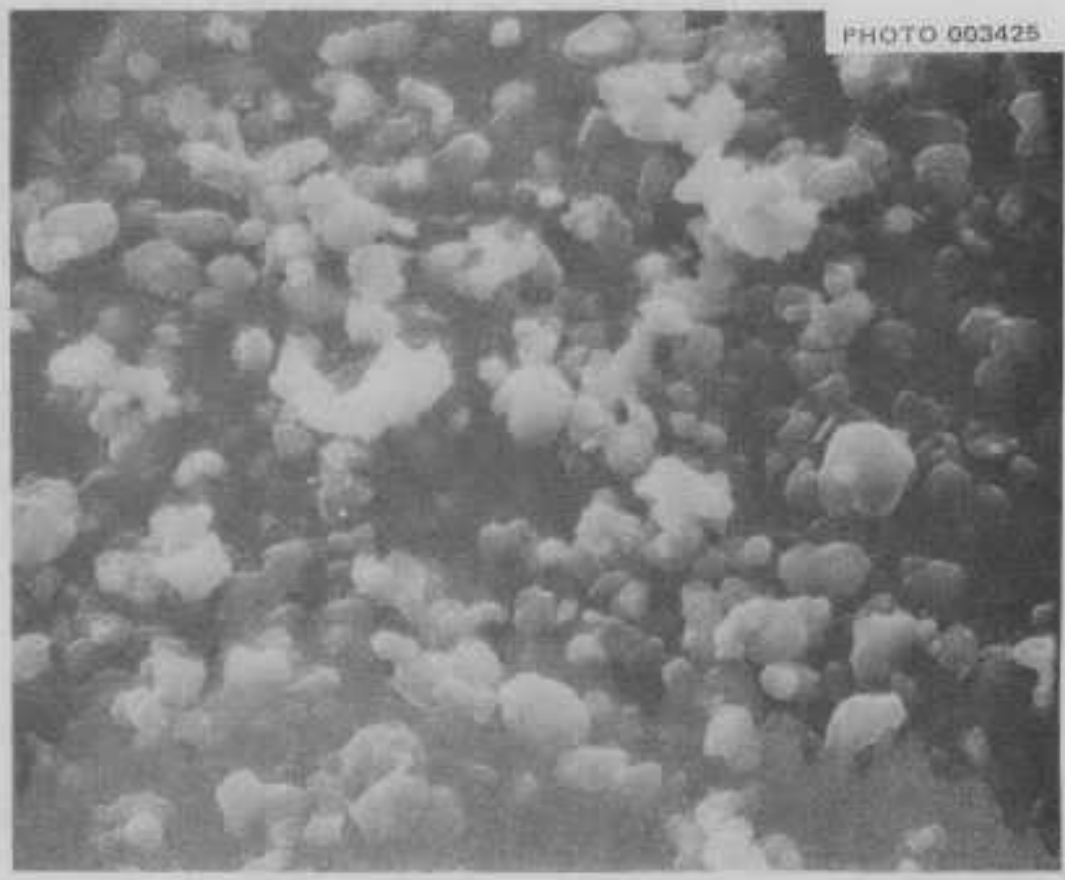

$1200 \times$

Fie. 3.8-6. Scanning electron micrograph (top left), iron map (top right), and sillicon map (bottom) from stage 2 , 6 G sampler. Magnification = $1200 x$. 
$X$-ray diffraction patterns of the particle sample ${ }^{5}$ from stage 2 shown in Fig. 3.2-6 did reveal iron present as $\mathrm{Fe}_{3} \mathrm{C}$ and definitely not as $\mathrm{Fe}_{2} \mathrm{O}_{3}$ or $\mathrm{Fe}_{3} \mathrm{O}_{4}$ which normally produce strong, characteristic lines. This observation held true for all the dust samples taken from the CB sampler impactor stages and filter unit. In adaition, diffraction patterns indicate the presence of graphite in the sample; amorphous carbon would not be detected.

The combined SEM-XRF and XRI-XRF spectra reveal the range of compositions for the CB sample dust shown in Table 3.2-8. Note that neither method would reveal oxygen or carbon. Uranium or thorium would have been seen in concentrations above $\sim 50 \mathrm{ppm}$.

Table 3.2-8. Composite SEM-XRF and XRI-XRF analyses of $\mathrm{CB}$ sampler dust ${ }^{\mathrm{a}}$

\begin{tabular}{ll} 
Iron & Major component, 50-80\% \\
Silicon & Major component, 20-50\% \\
Sulfur & $1-5 \%$ \\
Titanium & Present but less than $0.1 \%$ \\
Chromium & Present but less than $0.1 \%$ \\
Calcium & Present but less than $0.1 \%$ \\
Manganese & Present but less than $0.1 \%$ \\
Nickel & Present but less than $0.1 \%$ \\
Zinc & Present but less than $0.1 \%$ \\
Copper & Present but less than $50 \mathrm{ppm}(0.1 \%)$ \\
Molybdenum & Present but less than $50 \mathrm{ppm}$ \\
Gold & Present but less than $50 \mathrm{ppm}$ \\
Lead & Present but less than $50 \mathrm{ppm}$ \\
Gellium ${ }^{\mathrm{c}}$ & Present but less than $50 \mathrm{ppm}$ \\
\hline
\end{tabular}

Grom ref. 5. Neither method reveals oxygen or carbon. bay be an artifact of sample preparation

Not seen in all samples. 
Figure 3.2-7 (d) shows an SEM micrograph of a sample from stage 3 at 2000X magnification. The particle sizes within the agglomerate range from $\sim 0.5$ to $2 \mu \mathrm{m}$. The predicted size range on this stage for heavy particles is 0.8 to $2.6 \mu \mathrm{m}$, as seen in Table $3.1-2$. The induced $\mathrm{x}$-ray spectrum for this field reveals the particles to be predominately iron and silicon (no carbon or oxygen would be indicated) with a small amount of sulfur. It is interesting to note that the individual particles appear to be strongly agglomerated. However, the agglomeration most likely occurred after the particles were collected, since the agglomerate size exceeds $0.05 \mathrm{~mm}$, whereas the individual particles are in the range predicted by the impactor design.

Figure 3.2-7 (a) shows a dust sample from impactor stage 4 magnified 100X. The particle at upper right is, in this case, grossly different from the range predicted by the impactor design. The particle is $\sim 0.5 \mathrm{~mm}$ in size compared to the cutoff size range of 0.3 to $0.7 \mu \mathrm{m}$ predicted for this stage. The associated iron and silicon maps shown in the figure again illustrate the close association of iron and silicon in these particles.

Figure 3.2-8 shows a more typical dust sample from stage 4, revealing strongly agglomerated particles in the range perhaps of 0.2 to $5 \mu \mathrm{m}$, which compares more favorably with the predicted range. However, the agglomeration is so strong that the individual particles are difficult to distinguish. The view at the right shows a field at 10,000X magnification; the agglomerate itself exceeds $0.2 \mathrm{~mm}$ in size.

The SEM observation of stage 5 also revealed occasional extremely oversized particles (one $0.2-\mathrm{mm}$ particle was observed), but generally, strong agglomerates composed of individual particles in the range of $\sim 0.2$ to $2 \mu \mathrm{m}$ were observed.

Analysis of dust on CD sampler impactor. ${ }^{6}$ A portion of the stage 2 plate of the CD sampler is shown in Fig. 3.2-9 (c) viewed at 1000X magnification; the central group of particles is magnified to 5000X and is shown in Fig. 3.2-9 (a). These appear to be the same type of iron-rich, flaky particles as seen on the $\mathrm{CB}$ sampler stages. The sizes range from 

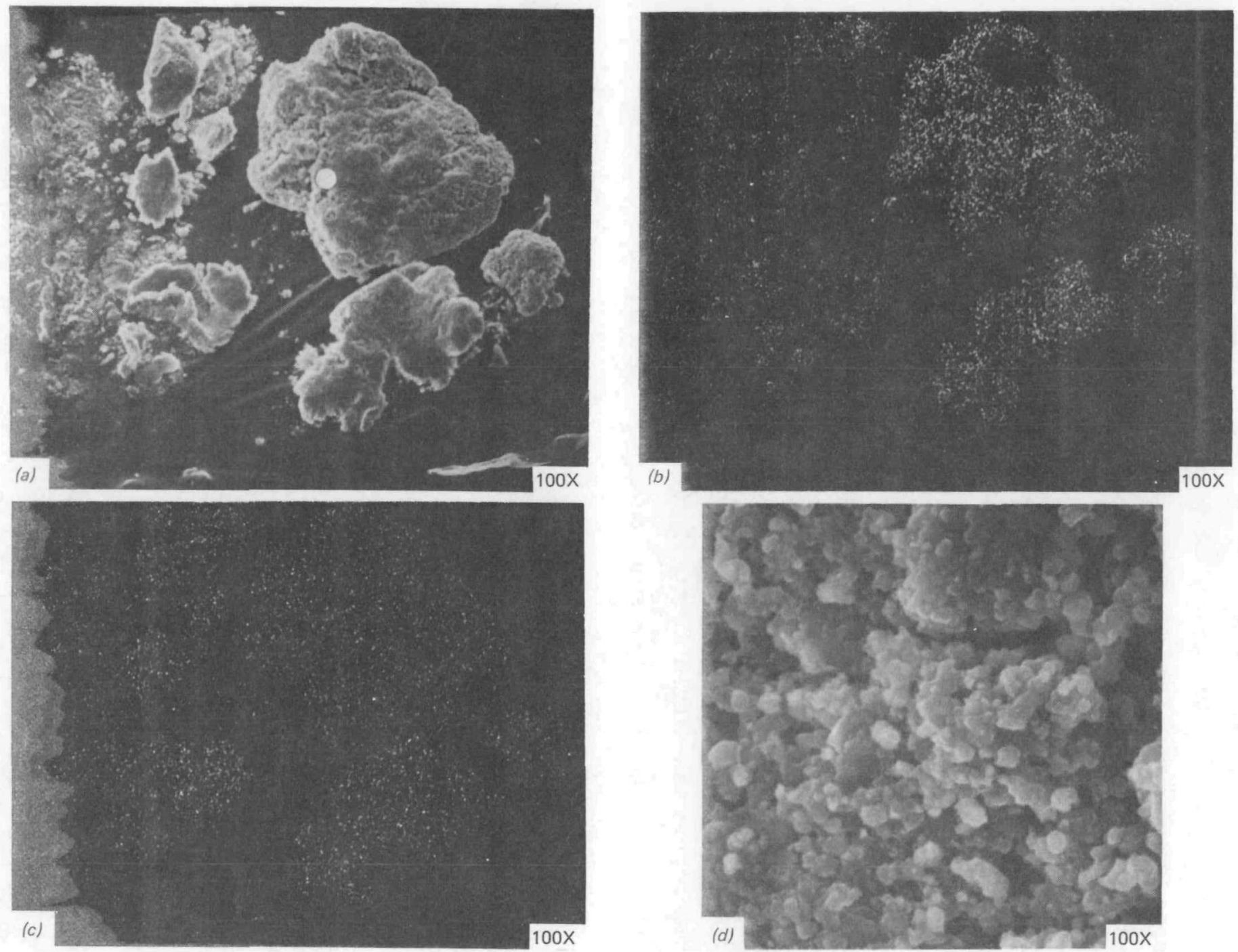

Fig. 3.2-7. Scanning electron micrographs of dust from CB coolant sampler. (a) Stage 4, 100X magnification; (b) corresponding iron map;

(c) corresponding silicon map; (d) stage 3, 2000X magnification. 

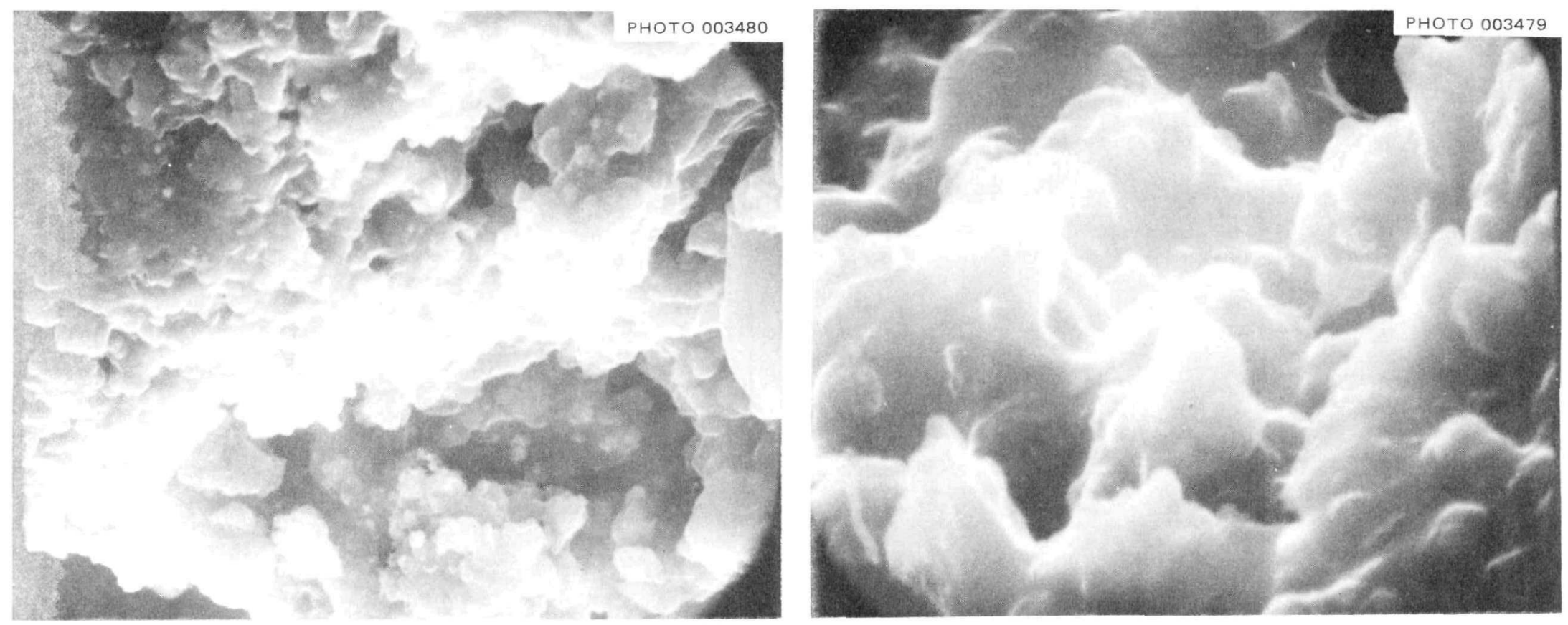

Fig. 3.2-8. Scanning electron micrograph of a dust sample from stage 4,

$C B$ sampler. Left magnification $=2000 \mathrm{X}$; right magnification $=10,000 \mathrm{X}$. 

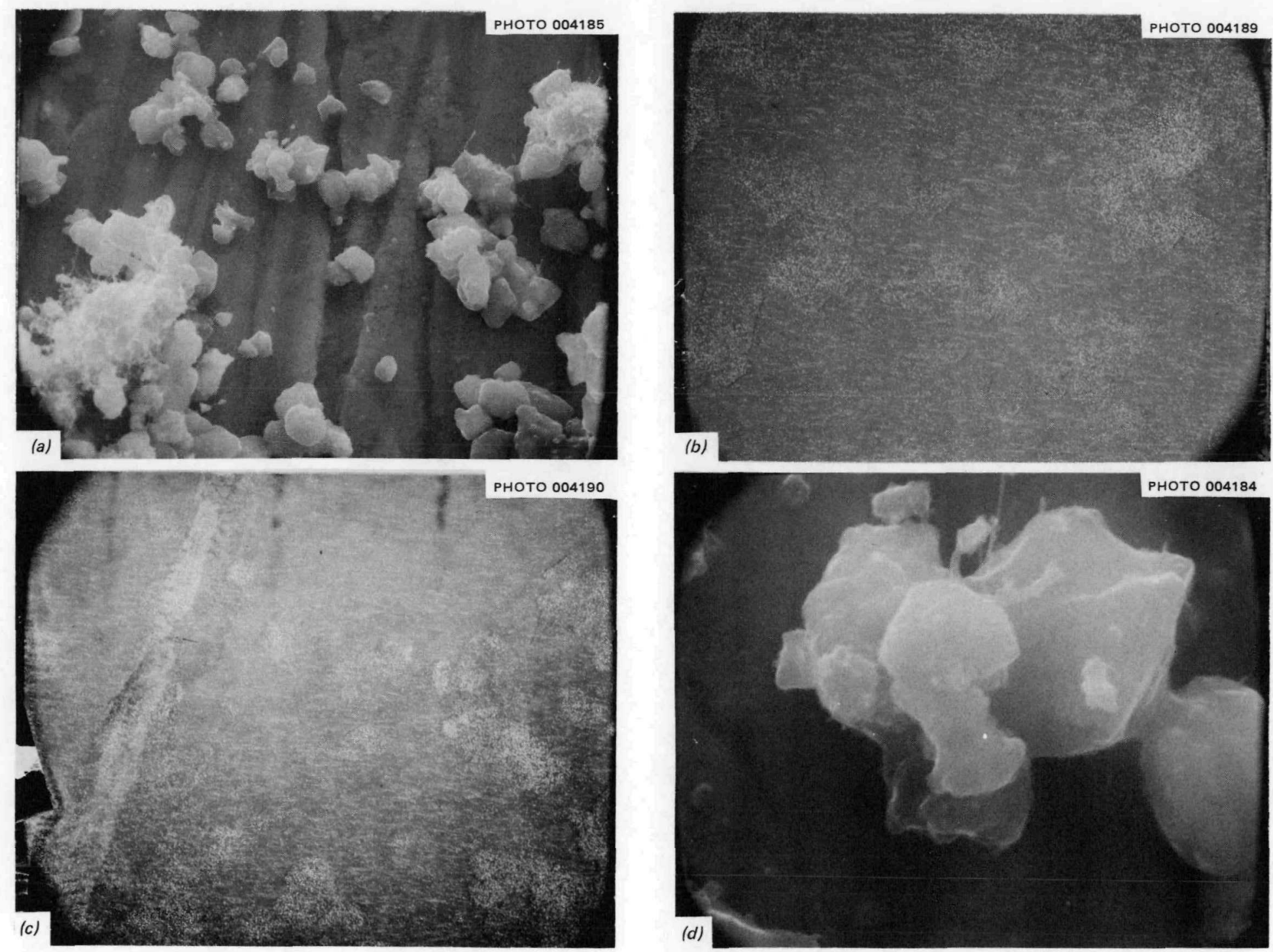

Fig. 3.2-9. Scanning electron micrographs of dist from CD sampler, impactor stage 2. (a) Typical field, 1000X magnification, (b) corresponding silicon map, (c) corresponding iron map, (d) central particle group at $5000 \mathrm{X}$ magnification. 
$\sim 2$ to $10 \mu \mathrm{m}$, compared with the predicted range of 5 to $20 \mu \mathrm{m}$ for heavy particles on stage 2 .

Figures 3.2-9 (b) and (d) are silicon and iron maps, respectively, which correspond to the 1000X magnification field shown in Fig. 3.2-9 (c). The significance here is that the silicon and iron locations are, again, closely correlated, as they were in the CB samples. Careful comparison of the three fields indicates that most particles contain both iron and silicon. However, the fibrous material in Fig. 3.2-9 (b) (1000X magnification) was demonstrated to contain silicon but no iron.

SEM-induced XRF spectra of the general stage 2 field indicated the two major observed constituents to be iron and silicon in about a one-toone atomic ratio. Sulfur is present at levels of $\sim 1 \%$ of the iron content and calcium, chromium, and manganese were observed at levels less than $1 \%$.

The major portion of the sample on stage 3 at low magnification is illustrated in Fig. 3.2-10 on the left, and a 200X magnification view is show on the right. Note here that the silicone grease coating that was applied to each stage of the CD sampler is clearly visible in the higher magnification view. The purpose of the grease application was to improve the adhesion of particulates on the impactor plates, which it may have done; however, it also served to mask the small samples collected on stages 2, 3, and 5. (Stage 4 contained essentially no dust, and the grease appeared to have been removed from stages $I$ and 2 by the action of the helium jet.) As expected, the SEM-XRF spectrum indicated exceptionally high silicon levels on these stages. For some reason, perhaps related to the silicon grease coating, the particle sizes on stage 3 appear to be much larger than expected.

In summary, the CD sampler stages contained much smaller amounts of particulates which were partially masked by silicon grease. The x-ray diffraction analysis of the dust on stage 1 of the CD sampler indicated a composition in the range of 40 to $80 \%$ elemental iron and 5 to $25 \%$ graphite. The particulates on stage 2 were shown to consist of 25 to $50 \%$ elemental iron, 30 to $70 \% \mathrm{FeO}(\mathrm{OH})$, and 5 to $25 \%$ graphite. Thus, the dust 

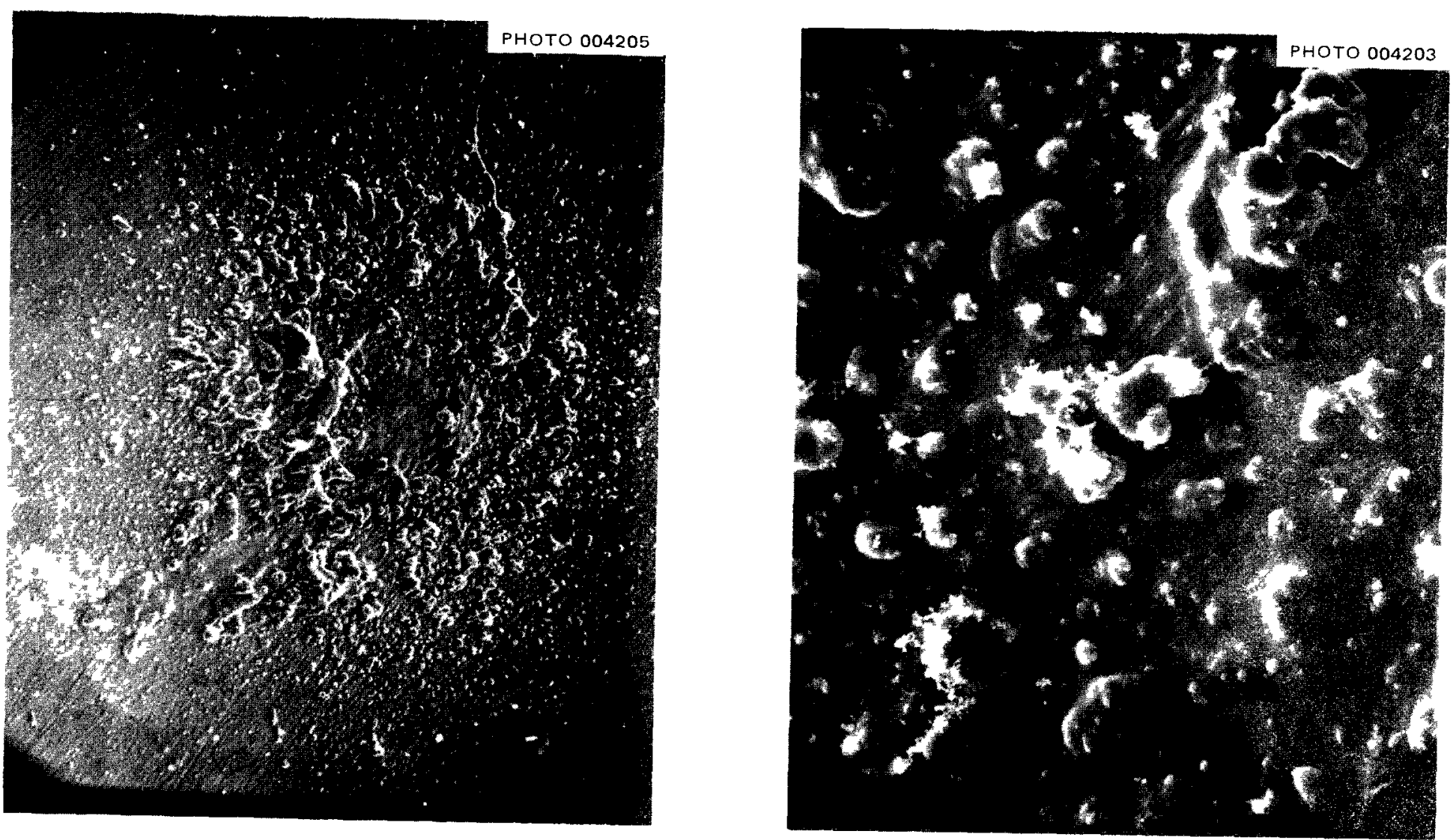

Fig. 3.2-10. Scanning electron micrograph, stage 3 of CD sampler. Left - overall view of sample at $20 \mathrm{X}$ magnification; right - $200 \mathrm{X}$ magnification. 
collected on the CD impactor plates diftered sipilificanty from the larger quantity collected on the previous CB sarrplex.

Summary of coolant sampler dust analvses. Analysis of the particulates collected on the CB-sampler impactor stages by means of SFM-XRF', XRI-XRF, and X-ray diffrgction indieated the maior constituents to be graphite, $F C_{3} C$, and an unknown iron-silicon compound which left no diffraction pattern. An apreciable amount of sulfur vas ioun ( 1 to 5\%). The particulace composition did not vary significantly beuween stages.

If the strong degree of agglomeration that was observed occurred after sampling, then the impactor functioned as a size classifier in a manner predicted by the design procedure, assuming a hcavy particle $\left(\rho_{p}=\sim 5 \mathrm{~g} / \mathrm{cm}^{3}\right)$. However, enomously oversized particles were occasionally observed.

The CD sampler stages contained much smaller amounts of dust which were partially masked by silicone grease applied to improve sticking. The dust material appeared to be basically different from the material observed in the $\mathrm{CB}$ sampler stages, containing elemental iron and no $\mathrm{Fe}_{3} \mathrm{C}$.

Additional chemical analyses of dust material taken from the cyclone separators in the primary loop and "sooty" deposits removed from the surface of fuel elements appear in sect. 5. Also, it is interesting to compare the visual appearance of dust collected in the cyclone separators with that seen for the dust on the impactor stages show in Figs. 3.2-6 to $3 \cdot 2-8$.

\subsection{Axial Distributions in Sampler Diffusion Tubes and Nozzles}

\subsubsection{Diffusion tubes}

This section presents the observed, axial distributions of radionuclides in the coolant-sampler diffusion tubes. Table 3.3-1 lists the observed levels, in microcuries per centimeter, corrected to the scheduled reactor shutdown date of Jan. 8, 1972, for tubes A and B of upstream sampler, Cl. Activated corrosion products ${ }^{54} \mathrm{Mn},{ }^{59} \mathrm{Fe},{ }^{60} \mathrm{Co}$, and occasionally 
Table 3.3-1. Axial distribution in upstream sampler, $\mathrm{Cl}$, diffusion tubes $\mathrm{A}$ and $\mathrm{B}$ ( $\mu \mathrm{Ci} / \mathrm{cm}$, January 8,1972$)$

\begin{tabular}{|c|c|c|c|c|c|c|c|c|c|c|c|c|c|}
\hline \multirow[b]{2}{*}{$\begin{array}{c}\text { Segment } \\
\text { No. }{ }^{\mathrm{a}}\end{array}$} & \multicolumn{6}{|c|}{ Tube A } & \multicolumn{7}{|c|}{ Tube B } \\
\hline & ${ }^{54} \mathrm{Mn}$ & $59_{\mathrm{Fe}}$ & ${ }^{60} \mathrm{Co}$ & $110 m_{A g}$ & ${ }^{134} \mathrm{Cs}$ & ${ }^{137} \mathrm{Cs}$ & $54 \mathrm{Mn}$ & $59 \mathrm{Fe}$ & ${ }^{60} \mathrm{Co}$ & $90 \mathrm{Sr}$ & $110 \mathrm{~m}_{\mathrm{Ag}}$ & ${ }^{134} \mathrm{cs}$ & $\overline{137} \mathrm{Cs}$ \\
\hline 1 & $1.58 \mathrm{~N}^{\mathrm{b}}$ & $3.12 \mathrm{~N} 4$ & $1.99 \mathrm{~N}_{4}$ & $1.96 \mathrm{N4}$ & $3.23 \mathrm{N4}$ & $1.25 \mathrm{N3}$ & $1.36 \mathrm{N4}$ & $2.02 \mathrm{N4}$ & $1.85 \mathrm{~N} / 4$ & c & $3.03 \pi / 4$ & $8.15 \mathrm{~N} 4$ & $3.15 \mathrm{~N} 3$ \\
\hline 2 & $1.16 \mathrm{~N} 4$ & $1.87 \mathrm{~N} 4$ & $1.57 \mathrm{~N} 4$ & $1.88 \mathrm{~N} 4$ & $2.40 \mathrm{N4}$ & $9.46 \mathrm{~N} / 4$ & $8.29 \mathrm{N5}$ & ND & $1.32 \mathrm{~N} 4$ & 8.7 N5 & $2.45 \mathrm{~N} 4$ & $4.92 \mathrm{~N} 4$ & $1.83 \mathrm{~N}$ \\
\hline 3 & $9.59 \mathrm{N5}$ & $N D^{\mathrm{d}}$ & $1.37 \mathrm{~N} 4$ & $2.08 \mathrm{Nk}$ & $2.14 \mathrm{~N} 4$ & $8.62 \mathrm{N4}$ & & & & & & & \\
\hline 4 & 8.63 N5 & ND & $1.60 \mathrm{~N}_{4}$ & $2.30 \mathrm{N14}$ & $2.12 \mathrm{~N} 4$ & $8.13 \times 14$ & $9.05 \mathrm{~N} 5$ & $\mathrm{ND}$ & $1.28 \mathrm{N4}$ & $5.8 \mathrm{NS}$ & $2.42 N 4$ & $4.19 \mathrm{~N} 4$ & $1.62 \mathrm{~N} / 3$ \\
\hline 5 & $8.39 \mathrm{N5}$ & $\mathrm{ND}$ & $1.31 \mathrm{~N} / 4$ & $2.25 \mathrm{~N} / 4$ & $2.10 \mathrm{~N} 4$ & 7.68 N14 & 7.44 N5 & ND & $1.15 \mathrm{~N}^{4}$ & & $2.15 \mathrm{~N} 4$ & $3.58 \mathrm{~N}^{4}$ & $1.41 \mathrm{N3}$ \\
\hline 6 & $9.47 \mathrm{N5}$ & $\mathrm{ND}$ & $1.40 \mathrm{N4}$ & $2.26 \mathrm{N4}$ & $2.30 \mathrm{~N} 4$ & $9.01 \mathrm{NT}$ & $9.57 \mathrm{N5}$ & ND & $1.32 \mathrm{N4}$ & 8.4 N5 & $2.59 \mathrm{NL}$ & $3.71 \mathrm{~N} 4$ & $1.51 \mathrm{NB}$ \\
\hline 7 & $8.91 \mathrm{N5}$ & $\mathrm{ND}$ & $1.46 \mathrm{~N} / 4$ & $2.15 \mathrm{~N} 4$ & $2.13 \mathrm{Nth}$ & $9.08 \mathrm{NL}$ & $1.26 \mathrm{N4}$ & $3.40 \mathrm{~N} / 4$ & $1.66 \mathrm{~N} 4$ & & $3.02 \mathrm{~N} 4$ & $4.08 \mathrm{N4}$ & $1.58 \mathrm{N3}$ \\
\hline 8 & $8.59 \mathrm{N5}$ & $1.90 \mathrm{~N} 4$ & $1.33 \mathrm{~N}^{4}$ & $2.40 \mathrm{~N} 4$ & $1.88 \mathrm{~N} 4$ & $7.81 \mathrm{~N} 4$ & $1.71 \mathrm{~N}_{4}$ & ND & $1.90 \mathrm{~N} 4$ & $1.42 \mathrm{~N}^{4}$ & $3.02 \mathrm{~N}^{4}$ & $4.41 \mathrm{N4}$ & $1.75 \mathrm{~N} 3$ \\
\hline 9 & 7.17 N5 & ND & $1.19 \mathrm{~N}^{4}$ & $2.50 \mathrm{~N} 4$ & $2.05 \mathrm{~N} 4$ & $7.67 \mathrm{N4}$ & $1.44 \mathrm{~N}^{4}$ & ND & $1.96 \mathrm{~N} / 4$ & & $2.33 \mathrm{~N} 4$ & $3.76 \mathrm{~N} 4$ & $1.54 \mathrm{N3}$ \\
\hline 10 & $7.15 \mathrm{~N} 5$ & $\mathrm{ND}$ & $1.19 \mathrm{~N} 4$ & $2.5 \mathrm{~N} 4$ & $2.05 \mathrm{~N} 4$ & $7.67 \mathrm{~N} 4$ & $1.40 \mathrm{~N} 4$ & ND & $2.16 \mathrm{N4}$ & $1.04 \mathrm{~N} 4$ & $2.74 \mathrm{~N} 4$ & $3.66 \mathrm{N4}$ & $1.45 \mathrm{~N} 3$ \\
\hline 11 & $8.63 \mathrm{N5}$ & $1.35 \mathrm{~N} 4$ & $1.84 \mathrm{~N} 4$ & $2.6 \mathrm{~N} 4$ & $2.26 \mathrm{~N} 4$ & $8.85 \mathrm{~N} 4$ & 1. $27 \mathrm{~N} 4$ & $1.48 \mathrm{~N} 4$ & $1.73 \mathrm{N4}$ & & $2.81 \mathrm{N4}$ & $3.57 \mathrm{Nh}$ & $1.38 \mathrm{N3}$ \\
\hline 12 & 8.32 N5 & $1.40 \mathrm{~N} 4$ & $1.30 \mathrm{NI}$ & $2.70 \mathrm{~N} 4$ & $2.41 \mathrm{~N} 4$ & $9.22 \mathrm{N4}$ & & & & $1.04 N 4$ & & & \\
\hline 13 & $2.84 \mathrm{N5}$ & $\mathrm{ND}$ & $8.35 \mathrm{~N} 5$ & $2.46 \mathrm{~N}_{4}$ & $1.05 \mathrm{~N} 4$ & $4.18 \mathrm{~N} 4$ & $1.25 \mathrm{~N} 4$ & $\mathrm{ND}$ & $1.62 \mathrm{~N} 4$ & & $2.68 N^{2}$ & $3.33 \mathrm{~N} 4$ & $1.33 \mathrm{~N} 3$ \\
\hline 14 & $9.90 \mathrm{N5}$ & ND & $1.40 \mathrm{~N} 4$ & $2.82 \mathrm{N4}$ & $2.45 \mathrm{~N} 4$ & $9.69 \mathrm{NL}$ & $1.31 \mathrm{~N} 4$ & ND & $1.58 \mathrm{~N} 4$ & $9.0 \mathrm{N5}$ & $2.58 \mathrm{~N} 4$ & $3.29 \mathrm{~N}_{4}$ & $1.27 \mathrm{~N}$ \\
\hline 15 & $1.09 \mathrm{N4}$ & $3.39 \mathrm{~N} 4$ & $1.52 \mathrm{~N} 4$ & $2.80 \mathrm{~N} 4$ & $2.56 \mathrm{~N} 4$ & 9.87 NL & $1.25 \mathrm{~N} 4$ & $\mathrm{ND}$ & $1.87 \mathrm{~N} 4$ & & $2.28 \mathrm{N4}$ & $3.19 \mathrm{~N} 4$ & $1.23 \mathrm{~N} 3$ \\
\hline 16 & $8.92 \mathrm{~N} 5$ & ND & $1.28 \mathrm{~N} 4$ & $2.60 \mathrm{~N}_{4}$ & $2.50 \mathrm{~N} 4$ & $9.71 \mathrm{~N} 4$ & $1.20 \mathrm{~N}^{4}$ & ND & $1.65 \mathrm{~N} 4$ & $1.04 \mathrm{~N} 4$ & $2.79 \mathrm{~N}^{4}$ & $3.15 \mathrm{~N}^{4}$ & $1.28 \mathrm{N3}$ \\
\hline 17 & $1.00 \mathrm{~N} 4$ & ND & $1.50 \mathrm{~N} 4$ & $2.37 \mathrm{~N} 4$ & $2.24 \mathrm{~N} / 4$ & $9.28 \mathrm{~N} 4$ & $2.56 \mathrm{N3}$ & $2.95 \sqrt{3}$ & $3.39 \mathrm{N3}$ & & $6.08 \mathrm{NB}$ & $6.67 \mathrm{~N} 3$ & $2.54 \mathrm{N2}$ \\
\hline 18 & 9.26 N5 & $2.04 \mathrm{N4}$ & $1.28 \mathrm{~N} 4$ & $2.75 \mathrm{~N} 4$ & $2.76 \mathrm{~N} 4$ & $1.09 \mathbb{N 3}$ & $1.15 \mathrm{~N} 4$ & $2.70 \mathrm{~N} 4$ & $1.66 \mathrm{N4}$ & & $2.81 \mathrm{~N} 4$ & $1.60 \mathrm{~N}^{4}$ & $1.18 \mathrm{~N} 3$ \\
\hline 19 & 9.62 N5 & ND & $1.47 \mathrm{~N} 4$ & $2.77 \mathrm{~N}_{4}$ & $2.81 \mathrm{~N}$ & $1.11 \mathrm{NB}$ & $1.15 \mathrm{~N} 4$ & ND & $1.66 \mathrm{~N} 4$ & & $2.74 \mathrm{~N} / 4$ & $3.05 \mathrm{N4}$ & $1.18 \mathrm{~N} 3$ \\
\hline 20 & ND & $\mathrm{ND}$ & ND & $\mathrm{ND}$ & & & $9.08 \mathrm{~N} 5$ & ND & $1.33 \mathrm{~N} 4$ & $7.2 \mathrm{~N} 5$ & $2.83 \mathrm{~N} / 4$ & $2.33 \mathrm{~N} 4$ & $8.87 \mathrm{N4}$ \\
\hline 21 & $7.18 \mathrm{~N} 5$ & $\mathrm{ND}$ & $1.19 \mathrm{~N} 4$ & $2.75 \mathrm{~N} 4$ & $2.87 \mathrm{N4}$ & $1.11 \mathrm{~N} 3$ & & & & & & & \\
\hline 22 & $\mathrm{ND}$ & $\mathrm{ND}$ & $\mathrm{ND}$ & ND & & & $6.81 \mathrm{N5}$ & $\mathrm{ND}$ & $1.35 \mathrm{N5}$ & & $2.35 \mathbb{N 4}$ & $1.99 \mathrm{~N} 4$ & $7.82 \mathrm{~N} 4$ \\
\hline 23 & $6.17 \mathrm{N5}$ & $2.62 \mathrm{N4}$ & $1.05 \mathrm{~N} / 4$ & $2.26 \mathbb{N 4}$ & $3.08 \mathrm{NH}$ & $1.21 \mathrm{~N}$ & & & & & & & \\
\hline 24 & $5.36 \mathrm{N5}$ & $\mathrm{ND}$ & $9.63 \mathrm{N5}$ & $2.13 \mathrm{~N} 4$ & $3.29 \mathrm{~N} 4$ & $1.32 \mathrm{~N} 3$ & & & & & & & \\
\hline 25 & $8.18 \mathrm{N5}$ & $\mathrm{ND}$ & $1.07 \mathrm{~N} / 4$ & $2.50 \mathrm{~N} 4$ & $3.86 \mathrm{~N} / 4$ & $1.52 \mathrm{N3}$ & & & & & & & \\
\hline 26 & & ND & $1.02 \mathrm{~N} 4$ & $2.38 \mathrm{~N} 4$ & $4.47 \mathrm{~N} 4$ & $1.74 \mathrm{N3}$ & & & & & & & \\
\hline
\end{tabular}

Each segment $2 \mathrm{~cm}$ long, numbered from inlet.

$b_{1.0} \mathrm{~N}^{4}$ indicates $1.0 \times 10^{-4}$.

Blank signifies not measur:d.

$\mathrm{a}_{\mathrm{ND}}$ indicates not detected. 
${ }^{65} \mathrm{Zn}$ (not listed) were observed, as well as fission products $110 \mathrm{~m} \mathrm{Ag}$, ${ }^{134} \mathrm{Cs}$, and ${ }^{137} \mathrm{Cs}$. Diffusion tube $\mathrm{C}$ from this sampler was sent to GAC for analysis.

Table 3.3-2 lists the observed distributions in tubes $A, B$, and $C$ of upstream sampler, CA, of fission products ${ }^{90} \mathrm{Sr}, 110 \mathrm{~m} \mathrm{Ag},{ }^{13 I_{I}},{ }^{134} \mathrm{Cs}$, and ${ }^{137} \mathrm{Cs}$. Table 3.3-3 lists distributions for these three tubes of corrosion products ${ }^{54} \mathrm{Mn},{ }^{59} \mathrm{Fe},{ }^{60} \mathrm{Co}$, and ${ }^{65} \mathrm{Zn}$. All were corrected to the reactor shutdown date of May 18, 1973. Tube $C$ of this sampler was completely dissolved for a ${ }^{90} \mathrm{Sr}$ determination. A total of $2.03 \times 10^{-3}$ $\mu \mathrm{C} i$ of ${ }^{90} \mathrm{Sr}$ was found in tube $\mathrm{C}$. Tubes $\mathrm{F}$ and $\mathrm{F}$ were not processed.

Tables 3.3-4 and 3.3-5 record the observed axial distributions in tubes $A$ and $B$ of the downstream sampler, CB, corrected to May I8, 1973.

Tables 3.3-6 and 3.3-7 record the observed distributions in tubes A through D of the upstream sampler, CC, corrected to May 28, 1974. Tables 3.3-8 and 3.3-9 record the distributions in tubes $A, B$, and $C$ of the downstream sampler, $\mathrm{CD}$, corrected to the same date.

\subsubsection{Axial distributions on sampler nozzles}

The amounts of radionuclides deposited in the sampler nozzles were found to be an appreciable fraction of the amount found on the diffusion tubes, as already noted in Tables 3.2-2 to 3.2-6. Since the nozzles are, effectively, diffusion tubes operating with a high flow rate condition, deposition profiles along the nozzles may contain additional information regarding the nature of the circulating activity. Accordingly, the nozzles were cleaned externally and cut into five segments of equal length; each segment was analyzed for ${ }^{134} \mathrm{Cs}$ and ${ }^{137} \mathrm{Cs}$. The measured distributions of ${ }^{134} \mathrm{Cs}$ and ${ }^{137} \mathrm{Cs}$ on the $\mathrm{CA}, \mathrm{CB}, \mathrm{CC}$, and $\mathrm{CD}$ samplex nozzles are presented in Table 3.3-10. 
Table 3.3-2. Axial distribution of radionuclides in upstream sampler, $C A$, diffusion tubes $A$, $B$, and $C^{a}$ $(\mu \mathrm{Cl} / \mathrm{cm}$, corrected to May 18,1973$)$

\begin{tabular}{|c|c|c|c|c|c|c|c|c|c|c|c|c|}
\hline \multirow[b]{2}{*}{$\begin{array}{l}\text { Segment } \\
\text { No. } b\end{array}$} & \multicolumn{4}{|c|}{ Tube A } & \multicolumn{5}{|c|}{ Tube B } & \multicolumn{3}{|c|}{ Tube $\mathrm{C}$} \\
\hline & $\overline{110 m_{A g}}$ & $131_{I}$ & ${ }^{134} \mathrm{Cs}$ & ${ }^{137} \mathrm{Cs}$ & ${ }^{90} \mathrm{Sr}$ & $110 \mathrm{~m}_{\mathrm{Ag}}$ & $13 I_{I}$ & ${ }^{13{ }^{4}} \mathrm{Cs}$ & ${ }^{137} \mathrm{Cs}$ & ${ }^{134} \mathrm{Cs}$ & ${ }^{137} \mathrm{Cs}$ & ${ }^{137} \mathrm{Cs} / /^{134} \mathrm{Cs}$ \\
\hline 1 & $1.97 \mathrm{~N}_{4} \mathrm{c}$ & $<4.74$ N5 & $3.13 \mathrm{N3}$ & $4.25 \mathrm{~N} 3$ & $6.2 \mathrm{~N} 4$ & $\mathrm{ND}^{\mathrm{d}}$ & ND & $4.27 \mathrm{~N} 3$ & $6.27 \mathrm{~N} 3$ & $3.80 \mathrm{~N} 3$ & $4.98 \mathrm{~N} 3$ & 1.32 \\
\hline 2 & e & ND & $3.47 \mathrm{N3}$ & $5.05 \mathrm{NB}$ & $2.7 \mathrm{NL}$ & ND & $8.21 \mathrm{N5}$ & $2.44 \mathrm{~N} 3$ & $4.05 \mathrm{~N} 3$ & $2.78 \mathrm{N3}$ & $4.12 \mathrm{N3}$ & 1.48 \\
\hline 3 & & $6.36 \mathrm{N5}$ & $1.68 \mathrm{NB}$ & $2.80 \mathrm{N3}$ & $9.2 \mathrm{N5}$ & 2.3 N5 & & & & $2.03 \mathrm{N3}$ & $3.65 \mathrm{N3}$ & 1.79 \\
\hline 4 & & & $1.21 \mathrm{~N} 3$ & $2.32 \mathrm{~N} 3$ & $1.09 \mathrm{N4}$ & & & $1.36 \mathrm{~N} 3$ & $2.92 \mathrm{N3}$ & $1.59 \mathrm{N3}$ & $3.24 \sqrt{3}$ & 2.03 \\
\hline 5 & $<1.92 \mathrm{~N} 3$ & $1.32 \mathrm{N4}$ & $1.00 \mathrm{N3}$ & $2.07 \mathrm{N3}$ & 6.1 N5 & $<5.5$ N6 & $1.87 \mathrm{~N} 4$ & $1.13 \mathrm{N3}$ & $2.71 \mathrm{NB}$ & $1.29 \mathrm{N3}$ & $2.74 \mathrm{N3}$ & 2.12 \\
\hline 6 & 17 N5 & & & & $\begin{array}{l}5.9 \mathrm{N5} \\
5\end{array}$ & & & & & $8.93 \mathrm{~N}^{2} 4$ & $2.16 \mathrm{N3}$ & 2.41 \\
\hline 7 & 1.17 N5 & $1.62 \mathrm{N4}$ & $6.75 \mathrm{N4}$ & $1.75 \mathrm{N3}$ & $\begin{array}{l}5.65 \mathrm{~N} 5 \\
5\end{array}$ & $<7.1$ a5 & & & & $7.65 \mathrm{~N} 4$ & $1.85 \mathrm{~N} 3$ & 2.42 \\
\hline 8 & $\leq 7.67$ N6 & $1.37 \mathrm{N4}$ & $5.37 \mathrm{~N}_{4}$ & $1.46 \mathrm{N3}$ & $5.65 \mathrm{N5}$ & & $1.88 \mathrm{~N} 4$ & $5.00 \mathrm{N4}$ & $1.93 \mathrm{N3}$ & $6.57 \mathrm{N4}$ & $1.73 \mathrm{~N} 3$ & 2.62 \\
\hline 9 & $<4.38 \times 6$ & $1.38 \mathrm{~N} 4$ & $5.06 \mathrm{~N} 4$ & $1.48 \mathrm{N3}$ & $4.5 \mathrm{~N} 5$ & $<7.9$ N5 & ND & $3.86 \mathrm{~N} 4$ & $1.04 \mathrm{N3}$ & $4.67 \mathrm{~N} 4$ & $1.41 \mathrm{~N} 3$ & 3.0 \\
\hline 10 & & & & & $3.8 \mathrm{N5}$ & & $1.13 \mathrm{~N} 4$ & $8.59 \mathrm{N5}$ & $3.22 \mathrm{N4}$ & $3.96 \mathrm{~N}_{4}$ & $1.22 \mathrm{~N} 3$ & 3.06 \\
\hline 11 & $<9.18 \mathrm{~N} 6$ & $1.36 \mathrm{~N} 4$ & $3.14 \mathrm{~N} 4$ & $1.07 \mathrm{~N} 3$ & $3.0 \mathrm{N5}$ & $<3.6 \mathrm{~N} 6$ & & & & $3.52 \mathrm{~N} 4$ & $1.01 \mathrm{N3}$ & 2.85 \\
\hline 12 & & & & & $1.67 \mathrm{~N} 5$ & & $1.43 \mathrm{~N} 4$ & $1.88 \mathrm{~N} 4$ & $5.99 \mathrm{~N} 4$ & $2.93 \mathrm{~N} / 4$ & $8.38 \mathrm{~N} 4$ & 2.85 \\
\hline 13 & $2.05 \mathrm{NT}$ & $1.36 \mathrm{~N} 4$ & $2.10 \mathrm{~N} 4$ & $7.13 \mathrm{~N}$. 14 & $1.67 \mathrm{~N} 5$ & $<4.9$ N6 & & ND & & $2.45 \mathrm{~N} 5$ & $8.02 \mathrm{~N} 4$ & $3.2 l_{4}$ \\
\hline 14 & - & & & & $1.3 \mathrm{N5}$ & & $1.23 \mathrm{~N} 4$ & $1.00 \mathrm{~N} 4$ & $3.69 \mathrm{~N} 4$ & $1.78 \mathrm{~N} 4$ & $6.57 \mathrm{N4}$ & 3.66 \\
\hline 15 & $<8.9$ N6 & $1.02 \mathrm{~N} 4$ & $1.36 \mathrm{~N} 4$ & $5.08 \mathrm{~N} / 4$ & $8.6 \mathrm{N6}$ & $<3.1$ N6 & & & & $1.44 \mathrm{~N} / 4$ & $5.28 \mathrm{~m} 14$ & 3.67 \\
\hline 16 & 00 & & & & $1.0 \mathrm{N5}$ & & $1.22 \mathrm{~N} 4$ & $1.13 \mathrm{~N} / 4$ & $4.63 \mathrm{~N}^{4}$ & $1.61 \mathrm{~N}_{4}$ & $5.69 \mathrm{~N}^{4}$ & 3.51 \\
\hline 17 & $<2.36 \mathrm{~N} 6$ & 9.65 N5 & $1.37 \mathrm{~N} / 4$ & $5.71 \mathrm{~N} 4$ & $1.05 \mathrm{~N} 5$ & $<4.616$ & & & & $1.69 \mathrm{~N} 4$ & $6.12 \mathrm{~N} 4$ & 3.59 \\
\hline 18 & (2) & 25 & & & $1.62 \mathrm{N5}$ & & $1.18 \mathrm{~N} 4$ & $1.48 \mathrm{~N} 4$ & $6.21 \mathrm{~N} 4$ & $2.00 \mathrm{~N} 4$ & $7.61 \mathrm{~N} 4$ & 3.78 \\
\hline 19 & $4.0 \mathrm{N6}$ & 7.94 N5 & $1.38 \mathrm{N4}$ & $6.21 \mathrm{~N}^{4}$ & $1.67 \mathrm{N5}$ & $5.11 \mathrm{N6}$ & & & & $1.87 \mathrm{~N}$ N. & $6.96 \mathrm{N4}$ & 3.7 \\
\hline $\begin{array}{l}20 \\
21\end{array}$ & $<3.62 N 6$ & $1.13 \mathrm{~N} 4$ & $1.50 \mathrm{~N} 4$ & $6.52 \mathrm{~N}_{4}$ & $\begin{array}{l}2.67 \mathrm{~N} 5 \\
3.25 \mathrm{~N}\end{array}$ & ND & $5.98 \mathrm{~N} 5$ & $1.34 \mathrm{~N} 4$ & $6.32 \mathrm{~N} 4$ & $1.96 \mathrm{N4}$ & $7.55 \mathrm{~N}^{4}$ & $\begin{array}{l}3.83 \\
4.0\end{array}$ \\
\hline $\begin{array}{l}21 \\
22\end{array}$ & 2.02 tro & & & & $\begin{array}{l}3.25 \mathrm{N5} \\
3.18 \mathrm{N5}\end{array}$ & & & & & $\begin{array}{l}1.57 \mathrm{~N} 4 \\
1.18 \mathrm{~N}\end{array}$ & $\begin{array}{l}6.30 \mathrm{N4} \\
5.35 \mathrm{N4}\end{array}$ & $\begin{array}{l}4.0 \\
4.53\end{array}$ \\
\hline $\begin{array}{l}22 \\
23\end{array}$ & $1.14 \mathrm{N5}$ & $1.66 \mathrm{~N} 4$ & $1.42 \mathrm{~N} 4$ & $5.98 \mathrm{~N}^{4}$ & $\begin{array}{l}3.18 \mathrm{N5} \\
6.0 \mathrm{~N} 5\end{array}$ & $1.67 \mathrm{N5}$ & $1.15 \mathrm{~N} 4$ & $1.26 \mathrm{~N} 4$ & $5.67 \mathrm{~N} 4$ & $2.03 \mathrm{~N} 4$ & $5.53 \mathrm{N4}$ & 2.72 \\
\hline 24 & 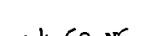 & & & & 7.9 N5 & ND & $6.36 \mathrm{N5}$ & $1.51 \mathrm{~N} 4$ & $6.55 \mathrm{~N} 4$ & $1.85 \mathrm{~N}_{4}$ & $7.55 \mathrm{~N} 4$ & 4.04 \\
\hline 25 & $<4.69 N 6$ & $5.91 \mathrm{~N} 5$ & $1.62 \mathrm{~N} 4$ & $6.66 \mathrm{~N}^{4}$ & & $9.37 \mathrm{N5}$ & & & & $2.48 \mathrm{N4}$ & $1.08 \mathrm{N3}$ & 4.32 \\
\hline 26 & $2.23 \mathrm{N5}$ & $3.96 \mathrm{N5}$ & $2.92 \mathrm{~N} 4$ & $1.05 \mathrm{N3}$ & $1.5 \mathrm{~N}^{4}$ & & & & & $3.64 \mathrm{~N} / 4$ & $1.60 \mathrm{~N} 3$ & 4.37 \\
\hline
\end{tabular}

${ }^{2}$ Tube $\mathrm{D}$ was dissolved, and ${ }^{90} \mathrm{Sr}$ was determined to be $2.03 \mathrm{~N} 3 \mu \mathrm{Cl}$. Tubes $\mathrm{E}$ and $\mathrm{F}$ were not processed.

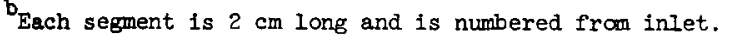

$\mathrm{c}_{1.0 \mathrm{~N} 4}$ denotes $1.0 \times 10^{-4}$.

a $\mathrm{ND}$ indicates not detected.

e Blank signifies not measured. 
Table 3.3-3. Distribution of activated corrosion products in upstream sampler, CA, diffusion tubes

$$
(\mu \mathrm{Ci} / \mathrm{cm}, \text { May 18, 1973) }
$$

\begin{tabular}{|c|c|c|c|c|c|c|c|c|}
\hline \multirow{2}{*}{$\begin{array}{c}\text { Segment } \\
\text { No. }{ }^{\mathrm{a}}\end{array}$} & \multicolumn{4}{|c|}{ Tube A } & \multicolumn{4}{|c|}{ Tube B } \\
\hline & ${ }^{54} \mathrm{Mn}$ & ${ }^{59} \mathrm{Fe}$ & ${ }^{60} \mathrm{Co}$ & ${ }^{65} \mathrm{Zn}$ & ${ }^{54} \mathrm{Mn}$ & ${ }^{59} \mathrm{Fe}$ & ${ }^{60} \mathrm{Co}$ & ${ }^{65} \mathrm{zn}$ \\
\hline$\frac{1}{2}$ & $2.07 \mathrm{N4}$ & 4.82 N5 & $\mathrm{ND}^{\mathrm{b}}$ & $3.38 \mathrm{~N} 5$ & c & & & \\
\hline $\begin{array}{l}3 \\
4\end{array}$ & & & & & $6.91 \mathrm{~N} 5$ & $1.15 \mathrm{~N} 4$ & $1.45 \mathrm{~N} 4$ & $7.45 \mathrm{~N} 6$ \\
\hline $\begin{array}{l}5 \\
6\end{array}$ & 5.5 N5 & $1.54 \mathrm{~N} 4$ & $\mathrm{ND}$ & $2.97 \mathrm{~N} 4$ & $4.76 \mathrm{~N} 5$ & 6.62 N5 & $1.29 \mathrm{~N} 4$ & $8.36 \mathrm{~N} 6$ \\
\hline $\begin{array}{l}7 \\
8\end{array}$ & $\begin{array}{l}2.4 \mathrm{~N} 5 \\
1.39 \mathrm{~N} 5\end{array}$ & $\begin{array}{ll}1.27 & \mathrm{~N} 4 \\
7.77 & \mathrm{~N} 5\end{array}$ & $\begin{array}{l}\text { ND } \\
8.82 \text { N5 }\end{array}$ & $\begin{array}{l}9.28 \text { N5 } \\
3.40 \text { N5 }\end{array}$ & $4.16 \mathrm{~N} 5$ & $4.15 \mathrm{~N} 5$ & ND & $1.09 \mathrm{~N} 5$ \\
\hline $\begin{array}{r}9 \\
10\end{array}$ & 1.64 N5 & $7.9 \mathrm{~N} 5$ & 7.19 N5 & $1.96 \mathrm{~N} 5$ & 2.66 N5 & $2.61 \mathrm{~N} 5$ & $1.12 \mathrm{~N} 4$ & 1.49 N5 \\
\hline $\begin{array}{l}11 \\
12\end{array}$ & $1.74 \mathrm{~N} 5$ & $7.49 \mathrm{~N} 5$ & $\mathrm{ND}$ & $1.65 \mathrm{~N} 5$ & $1.51 \mathrm{~N} 5$ & $4.29 \mathrm{~N} 5$ & $5.25 \mathrm{~N} 5$ & $6.29 \mathrm{~N} 6$ \\
\hline $\begin{array}{l}13 \\
14\end{array}$ & $2.01 \mathrm{N5}$ & 7.29 N5 & $8.19 \mathrm{~N} 5$ & $8.16 \mathrm{~N} 6$ & $1.21 \mathrm{~N} 5$ & $3.45 \mathrm{~N} 5$ & $\mathrm{ND}$ & $8.49 \mathrm{~N} 6$ \\
\hline $\begin{array}{l}15 \\
16\end{array}$ & 7.81 N5 & 7.07 N5 & & $8.13 \mathrm{~N} 6$ & $1.44 \mathrm{~N} 5$ & $4.78 \mathrm{~N} 5$ & $5.36 \mathrm{~N} 5$ & $6.31 \mathrm{~N} 6$ \\
\hline $\begin{array}{l}19 \\
20\end{array}$ & $2.61 \mathrm{N5}$ & $3.61 \mathrm{~N} 5$ & $7.01 \mathrm{N5}$ & $5.81 \mathrm{~N} 6$ & 1.79 N5 & 8.33 N5 & $6.23 \mathrm{~N} 5$ & $5.78 \mathrm{~N} 5$ \\
\hline $\begin{array}{l}21 \\
22\end{array}$ & $4.42 \mathrm{~N} 5$ & $8.70 \mathrm{NG}$ & $1.29 \mathrm{~N} 4$ & $5.93 \mathrm{~N} 6$ & & & & \\
\hline $\begin{array}{l}23 \\
24\end{array}$ & $6.04 \mathrm{~N} 5$ & $5.15 \mathrm{~N} 5$ & $1.35 \mathrm{~N} 4$ & $9.55 \mathrm{~N} 6$ & $4.25 \mathrm{~N} 5$ & 9.24 N5 & $9.54 \mathrm{~N} 5$ & 2.6 N4 \\
\hline $\begin{array}{l}25 \\
26\end{array}$ & $\begin{array}{l}7.71 \text { N5 } \\
1.27 \mathrm{~N} 4\end{array}$ & $\begin{array}{l}1.08 \mathrm{N5} \\
2.24 \mathrm{~N} 4\end{array}$ & $\begin{array}{l}\text { ND } \\
2.30 \mathrm{~N} 4\end{array}$ & $\begin{array}{l}7.05 \mathrm{~N} 6 \\
6.50 \mathrm{~N} 6\end{array}$ & $1.44 \mathrm{~N} 4$ & $2.77 \mathrm{~N}_{4}$ & $2.49 \mathrm{~N}_{4}$ & $1.03 \mathrm{~N} 4$ \\
\hline
\end{tabular}

Each segment is $2 \mathrm{~cm}$ and is numbered from inlet.

$\mathrm{b}_{\mathrm{ND}}$ indicates not detected.

$c_{\text {Blank indicates not measured. }}$ 
Table 3.3-4. Axial distribution of radionuclides in downstream sampler, $\mathrm{CB}$, diffusion tubes ( $\mu \mathrm{C} 1 / \mathrm{cm}$, May 18, 1973)

\begin{tabular}{|c|c|c|c|c|c|c|c|c|c|c|}
\hline \multirow[b]{2}{*}{$\begin{array}{l}\text { Segment } \\
\text { No. }\end{array}$} & \multicolumn{6}{|c|}{ Tube A } & \multicolumn{4}{|c|}{ Tube $B$} \\
\hline & ${ }^{90} \mathrm{Sr}$ & $110 m_{A B}$ & $131_{I}$ & ${ }^{134} \mathrm{cs}$ & ${ }^{137} \mathrm{Cs}$ & ${ }^{137} \mathrm{Cs} /{ }^{134} \mathrm{Cs}$ & $\overline{110 m_{A g}}$ & ${ }^{134} \mathrm{Cs}$ & ${ }^{137} \mathrm{Cs}$ & $137 \mathrm{Cs} /{ }^{134} \mathrm{Cs}$ \\
\hline$\frac{1}{2}$ & $8.85 \mathrm{N5}$ & $\begin{array}{l}6.59 \mathrm{~N}^{4} \\
6.82 \mathrm{~N}^{4}\end{array}$ & $1.39 \mathbb{N}^{4}$ & $\begin{array}{l}8.00 \mathrm{N4} \\
4.24 \mathrm{N4}\end{array}$ & $2.96 \mathrm{NB}$ & $\begin{array}{l}3.70 \\
3.35\end{array}$ & $\begin{array}{l}6.05 \mathrm{N4} \\
6.40 \mathrm{N4}\end{array}$ & $1.18 \mathrm{~N}$ & $4.22 \mathrm{N3}$ & 3.58 \\
\hline 3 & $6.90 \mathrm{N4}$ & $5.94 \times 14$ & $1.41 \mathrm{~N}$ & $2.89 \mathrm{~N} 4$ & $1.03 \mathrm{N3}$ & $\begin{array}{l}3.37 \\
3.35\end{array}$ & $6.15 \mathrm{N4}$ & $7.40 \mathrm{~N} / 4$ & $2.47 \sqrt{3}$ & 3.13 \\
\hline 4 & $6.20 \mathrm{N5}$ & $6.10 \mathrm{~N} 4$ & $1.73 \mathrm{N4}$ & $3.16 \mathrm{~N} 4$ & $1.12 \mathbb{N}$ & 3.54 & $6.49 \mathrm{~N} 4$ & $6.0 \mathrm{~N}$ & $2.12 \mathrm{NB}$ & 3.53 \\
\hline 5 & $4.41 \mathrm{N5}$ & $5.74 \mathrm{~N} 4$ & & $2.68 \mathrm{N4}$ & $9.63 \mathrm{N4}$ & 3.59 & $5.78 \mathrm{~N} 4$ & $5.15 \mathrm{N4}$ & $1.88 \mathrm{NB}$ & 3.65 \\
\hline 6 & $6.90 \mathrm{N5}$ & $6.14 \mathrm{N4}$ & $1.69 \mathrm{~N} 4$ & $3.21 \mathrm{~N} 4$ & $1.13 \mathrm{~N} 3$ & 3.52 & $\mathrm{ND}^{\mathrm{C}}$ & $4.91 \mathrm{~N}_{4}$ & $1.64 \mathrm{N3}$ & 3.34 \\
\hline 7 & & $6.39 \mathrm{~N} 4$ & $1.72 \mathrm{~N} 4$ & $2.45 \mathrm{~N}^{4}$ & $8.80 \mathrm{~N}$ & 3.59 & $5.82 \mathrm{~N} 4$ & $4.44 \mathrm{~N}^{4}$ & $1.55 \mathrm{NB}$ & 3.49 \\
\hline $\begin{array}{l}8 \\
9\end{array}$ & $\begin{array}{l}4.96 \mathrm{N5} \\
8.85 \mathrm{N5}\end{array}$ & $6.52 \mathrm{N4}$ & $1.99 \mathrm{N4}$ & $2.78 \mathrm{N4}$ & $1.00 \mathrm{N3}$ & 3.60 & ND & $4.16 \mathrm{~N}^{4}$ & $1.58 \mathrm{~N} 3$ & 3.79 \\
\hline 10 & $1.01 \mathrm{~N}^{4}$ & & & & & & $5.97 \mathrm{~N} 4$ & $2.80 \mathrm{~N} / 4$ & $1.08 \mathrm{~N} 3$ & 3.87 \\
\hline 11 & $7.95 \mathrm{~N} 5$ & $6.69 \mathrm{~N} 4$ & $2.53 \mathrm{~N} 4$ & $2.48 \mathrm{N4}$ & $9.04 \mathrm{~N} 4$ & 3.64 & $\mathrm{ND}$ & $3.65 \mathrm{~N}_{4}$ & $1.09 \mathrm{N3}$ & 2.99 \\
\hline $\begin{array}{l}12 \\
13\end{array}$ & $5.40 \mathrm{N5}$ & $6.48 \mathrm{N4}$ & $2.31 \mathrm{N4}$ & $1.83 \mathrm{~N}_{4}$ & $6.66 \mathrm{~N}^{4}$ & 3.64 & $5.94 \mathrm{ND}^{4}$ & $\begin{array}{l}3.17 \mathrm{Nh} \\
2.80 \mathrm{~N}\end{array}$ & $1.07 \mathrm{N3}$ & $\begin{array}{l}3.43 \\
3.57\end{array}$ \\
\hline 14 & $4.69 \mathrm{N5}$ & $6.71 \mathrm{Nh}$ & $1.96 \mathrm{N4}$ & $1.61 \mathrm{~N}^{4}$ & $5.80 \mathrm{~N} 4$ & 3.60 & ND & $2.37 \mathrm{~N}^{4}$ & $8.60 \mathrm{~N}^{4}$ & $\begin{array}{l}3.57 \\
3.63\end{array}$ \\
\hline $\begin{array}{l}15 \\
16\end{array}$ & $4.09 \mathrm{N5}$ & $6.69 \mathrm{N4}$ & $1.60 \mathrm{~N} 4$ & $1.60 \mathrm{N4}$ & $4.71 \mathrm{~N} 4$ & 2.94 & $6.00 \mathrm{~N} 4$ & $2.85 \mathrm{~N} 4$ & $8.60 \mathrm{~N} 4$ & 3.01 \\
\hline $\begin{array}{l}10 \\
17\end{array}$ & $\begin{array}{l}7.20 \mathrm{N5} \\
3.41 \mathrm{N5}\end{array}$ & $6.57 \mathrm{~N}^{4}$ & $1.42 \mathrm{~N}_{4}$ & $1.71 \mathrm{N4}$ & $5.32 \mathrm{~N} 4$ & 3.11 & ND & $2.63 \mathrm{N4}$ & $8.40 \mathrm{N4}$ & 3.19 \\
\hline 18 & 3.84 N5 & & & & & & $5.79 \mathrm{~N} 4$ & $2.31 \mathrm{N4}$ & $8.05 \mathrm{~N} 4$ & 3.48 \\
\hline 19 & $4.86 \mathrm{N5}$ & $6.65 \mathrm{~N} 4$ & $1.06 \mathrm{N4}$ & $1.97 \mathrm{~N} 4$ & $6.33 \mathrm{~N} 4$ & 3.21 & $6.47 \mathrm{N4}$ & $2.48 \mathrm{~N} / 4$ & $9.15 \mathrm{~N} 4$ & 3.69 \\
\hline $\begin{array}{l}20 \\
21\end{array}$ & $\begin{array}{l}5.80 \mathrm{N5} \\
7.75 \mathrm{N5}\end{array}$ & $6.29 \mathrm{~N} 4$ & 7.76 N5 & $2.11 \mathrm{NH}^{2}$ & $7.17 \mathrm{N4}$ & 3.40 & $\begin{array}{l}\text { ND } \\
5.26 \mathrm{N4}\end{array}$ & $\begin{array}{l}3.01 \mathrm{N4} \\
2.83 \mathrm{NL}\end{array}$ & $1.03 \mathrm{N3}$ & $\begin{array}{l}3.4 \\
3.3\end{array}$ \\
\hline 22 & $5.65 \mathrm{~N} 5$ & & & & & & ND & $2.65 \mathrm{N4}$ & $1.01 \mathrm{N3}$ & 3.8 \\
\hline $\begin{array}{l}23 \\
24\end{array}$ & $\begin{array}{l}5.75 \mathrm{~N} 5 \\
5.30 \mathrm{N5}\end{array}$ & $5.09 \mathrm{~N} 4$ & $4.38 \mathrm{N5}$ & $1.56 \mathrm{N4}$ & $5.41 \mathrm{N4}$ & 3.47 & $\begin{array}{l}\text { ND } \\
\text { ND }\end{array}$ & $\begin{array}{l}2.65 \mathrm{~N} 4 \\
2.60 \mathrm{~N} 4\end{array}$ & $\begin{array}{l}8.85 \mathrm{NL} \\
9.50 \mathrm{~N} 4\end{array}$ & $\begin{array}{l}3.33 \\
3.65\end{array}$ \\
\hline $\begin{array}{l}25 \\
26\end{array}$ & $\begin{array}{l}4.8 \mathrm{N5} \\
7.75 \mathrm{N5}\end{array}$ & $4.36 \mathrm{~N} 4$ & 3.57 N5 & $2.38 \mathrm{N4}$ & $8.43 \mathrm{~N} 4$ & 3.54 & $\begin{array}{c}4.99 \mathrm{N4} \\
\text { ND }\end{array}$ & $\begin{array}{l}2.75 \mathrm{~N} 4 \\
2.91 \mathrm{~N} 4\end{array}$ & $\begin{array}{l}9.65 \mathrm{N4} \\
9.50 \mathrm{~N} 4\end{array}$ & $\begin{array}{l}3.5 \\
3.26\end{array}$ \\
\hline
\end{tabular}

Each segment is $2 \mathrm{~cm}$ and is numbered from inlet.

Blank Indicates not measured.

$c_{\text {ND indicates not detected. }}$ 
Table 3.3-5. Distribution of activated corrosion products in downstream sampler, CB, diffusion tubes

$$
(\mu \mathrm{Ci} / \mathrm{cm} \text {, May 18, 1973) }
$$

\begin{tabular}{|c|c|c|c|c|c|c|c|c|}
\hline \multirow{2}{*}{$\begin{array}{c}\text { Segment } \\
\text { No. }{ }^{a}\end{array}$} & \multicolumn{4}{|c|}{ Tube A } & \multicolumn{4}{|c|}{ Tube $\mathrm{C}$} \\
\hline & $54 \mathrm{Mn}$ & $59_{\mathrm{Fe}}$ & ${ }^{60} \mathrm{Co}$ & ${ }^{65} \mathrm{Zn}$ & $54 \mathrm{Mn}$ & $59 \mathrm{Fe}$ & ${ }^{60} \mathrm{Co}$ & ${ }^{65} \mathrm{zn}$ \\
\hline 1 & $2.17 \mathrm{~N} 4$ & $3.09 \mathrm{~N} 4$ & $6.0 \mathrm{~N}^{4}$ & $\mathrm{ND}^{\mathrm{b}}$ & $2.74 \mathrm{~N} 4$ & $3.89 \mathrm{~N} 4$ & $5.68 \mathrm{~N} 4$ & $5.2 \mathrm{~N} 5$ \\
\hline 2 & 9.16 N5 & $1.06 \mathrm{~N} 4$ & $3.47 \mathrm{~N} 4$ & $9.08 \mathrm{~N} 5$ & $1.77 \mathrm{~N}_{4}$ & $3.51 \mathrm{~N}^{4}$ & $4.01 \mathrm{~N}_{4}$ & $4.48 \mathrm{~N} 5$ \\
\hline 3 & $6.18 \mathrm{~N} 5$ & $5.53 \mathrm{~N} 5$ & $1.64 \mathrm{~N} 4$ & ND & $1.78 \mathrm{~N} 4$ & $2.84 \mathrm{~N} 4$ & $3.46 \mathrm{~N} 4$ & 3.26 N5 \\
\hline 4 & 5.42 N5 & 6.35 N5 & $1.97 \mathrm{~N}^{4}$ & ND & $1.57 \mathrm{~N} 4$ & $2.38 \mathrm{N4}$ & $3.4 \mathrm{~N} 4$ & $\mathrm{ND}$ \\
\hline 5 & $\mathrm{c}$ & & & & $1.30 \mathrm{~N} 4$ & $2.29 \mathrm{~N} 4$ & $2.69 \mathrm{~N} 4$ & $4.17 \mathrm{~N} 5$ \\
\hline 6 & 6.99 N5 & $8.15 \mathrm{~N} 5$ & $2.11 \mathrm{~N} 4$ & 3.65 N5 & $\mathrm{ND}$ & $1.44 \mathrm{~N} 5$ & ND & $1.01 \mathrm{~N} 5$ \\
\hline 9 & $7.67 \mathrm{~N} 5$ & 8.81 N5 & $2.37 \mathrm{~N} 4$ & $\mathrm{ND}$ & & & & \\
\hline 10 & & & & & $9.05 \mathrm{~N} 5$ & $1.19 \mathrm{~N} 4$ & $1.80 \mathrm{~N} 4$ & $\mathrm{ND}$ \\
\hline 11 & 8.72 N5 & $1.42 \mathrm{~N} 4$ & $2.28 \mathrm{~N} 4$ & ND & & & & \\
\hline 12 & & & & & 9.31 N5 & $1.09 \mathrm{~N} 4$ & $1.96 \mathrm{~N} 4$ & $\mathrm{ND}$ \\
\hline 13 & $7.25 \mathrm{~N} 5$ & $8.22 \mathrm{N5}$ & $2.06 \mathrm{~N}^{4}$ & ND & & & & \\
\hline 14 & $5.58 \mathrm{N5}$ & $8.87 \mathrm{N5}$ & $2.14 \mathrm{~N}^{4}$ & $\mathrm{ND}$ & & & & \\
\hline 15 & $5.46 \mathrm{~N} 5$ & $8.50 \mathrm{~N} 5$ & $1.78 \mathrm{~N} 4$ & ND & 7.82 N5 & $1.64 \mathrm{~N} 4$ & $1.43 \mathrm{~N} 4$ & $\mathrm{ND}$ \\
\hline 16 & & & & & $1.0 \mathrm{~N} 4$ & $1.96 \mathrm{N4}$ & $1.72 \mathrm{~N} 4$ & $2.4 \mathrm{~N} 5$ \\
\hline 18 & & & & & $8.69 \mathrm{~N} 5$ & $1.54 \mathrm{~N}_{4}$ & $2.16 \mathrm{~N} 4$ & $\mathrm{ND}$ \\
\hline 19 & $7.94 \mathrm{~N} 5$ & $1.38 \mathrm{~N} 4$ & $2.09 \mathrm{~N}^{4}$ & $\mathrm{ND}$ & $1.17 \mathrm{~N} 4$ & $2.15 \mathrm{~N} 4$ & $2.08 \mathrm{~N}_{4}$ & $\mathrm{ND}$ \\
\hline $\begin{array}{l}20 \\
21\end{array}$ & $1.01 \mathrm{~N}_{4}$ & $1.80 \mathrm{~N} 4$ & $2.68 \mathrm{N4}$ & ND & $1.16 \mathrm{~N} 4$ & 237 N4 & $207 \mathrm{NL}$ & $102 \mathrm{N5}$ \\
\hline 22 & & & & & & $2.5 \perp 104$ & 2.07104 & 1.96 iv) \\
\hline $\begin{array}{l}23 \\
24\end{array}$ & 6.72 N5 & $1.14 \mathrm{~N} 4$ & $2.28 \mathrm{~N}^{4}$ & $3.26 \mathrm{~N} 5$ & & & & \\
\hline $\begin{array}{l}25 \\
26\end{array}$ & $1.07 \mathrm{~N} 4$ & $2.10 \mathrm{~N} 4$ & $2.72 \mathrm{~N} 4$ & $3.21 \mathrm{~N} 5$ & $1.13 \mathrm{~N} 4$ & $2.36 \mathrm{~N} 4$ & $1.84 \mathrm{~N} 4$ & ND \\
\hline
\end{tabular}

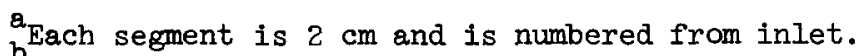

$\mathrm{bD}$ indicates not detected.

$\mathrm{c}_{\text {Blank indicates not measured. }}$ 
Table 3.3-6. Axial distribution of nuclides in upstream sampler, $\mathrm{CC}$, diffusion tubes

$(\mu \mathrm{Ci} / \mathrm{cm}$, May 28, 1974)

\begin{tabular}{|c|c|c|c|c|c|c|c|c|c|c|c|c|}
\hline \multirow{2}{*}{$\begin{array}{l}\text { Segment } \\
\text { No. }{ }^{8}\end{array}$} & \multicolumn{3}{|c|}{ Tube A } & \multicolumn{3}{|c|}{ Tube $B$} & \multicolumn{3}{|c|}{ Tube $\mathrm{C}$} & \multicolumn{3}{|c|}{ Tube D } \\
\hline & ${ }^{134} \mathrm{Cs}$ & $137 \mathrm{Cs}$ & ${ }^{137} \mathrm{cs} /{ }^{134} \mathrm{cs}$ & $13 I_{I}$ & ${ }^{134} \mathrm{Cs}$ & $137 \mathrm{Cs}$ & ${ }^{90} \mathrm{Sr}$ & ${ }^{134} \mathrm{Cs}$ & ${ }^{137} \mathrm{Cs}$ & ${ }^{90} \mathrm{Sr}$ & $134 \mathrm{Cs}$ & $137 \mathrm{Cs}$ \\
\hline 1 & $8.72 \mathrm{N2}$ & $6.33 \mathrm{~N} 2$ & 0.73 & b & & & $4.46 \mathrm{~N} 6$ & $7.64 \mathrm{~N} 2$ & $5.58 \mathrm{~N} 2$ & $4.29 \mathrm{~N} 6$ & $9.03 \mathrm{N2}$ & $6.66 \mathrm{~N} 2$ \\
\hline 2 & $5.63 \mathrm{N2}$ & $4.13 \mathrm{~N} 2$ & 0.73 & & & & & & & & & \\
\hline $\begin{array}{l}3 \\
4\end{array}$ & $\begin{array}{l}4.27 \mathrm{~N} 2 \\
4.71 \mathrm{~N} 2\end{array}$ & $\begin{array}{l}3.08 \mathrm{~N} 2 \\
3.46 \mathrm{~N} 2\end{array}$ & 0.72 & $N D^{\circ}$ & $4.6 \mathrm{~N} 2$ & $3.2: \mathrm{N} 2$ & $1.85 N 6$ & & & $1.13 \mathrm{~N} 6$ & & \\
\hline 5 & $5.67 \mathrm{~N} 2$ & $4.27 \mathrm{N2}$ & 0.75 & & $4.95 \mathrm{N2}$ & $3.16 \mathrm{N2}$ & $1.33 \mathrm{N6}$ & & & $2.17 \mathrm{N6}$ & & \\
\hline 6 & $4.78 \mathrm{N2}$ & $3.64 \mathrm{~N} 2$ & 0.76 & & & & & $2.22 \mathrm{~N} 2$ & $1.7 \mathrm{~N} 2$ & & $2.19 \mathrm{~N} 2$ & $1.64 \mathrm{~N} 2$ \\
\hline 7 & $3.15 \mathrm{~N} 2$ & $2.39 \mathrm{N2}$ & 0.76 & & & & $1.12 \mathrm{N6}$ & & & $7.29 \times 6$ & & \\
\hline 8 & $2.04 \mathrm{~N} 2$ & $2.66 \mathrm{N2}$ & 0.81 & $2.40 \mathrm{~N} 4$ & $1.87 \mathrm{~N} 2$ & $1.43 \mathrm{~N} 2$ & & & & & & \\
\hline 9 & $1.36 \mathrm{N2}$ & $1.03 \mathrm{N2}$ & $0.7 \overline{6}$ & & & & $1.12 N 6$ & & & $1.13 \mathrm{N6}$ & & \\
\hline 10 & $8.89 \mathrm{N3}$ & $6.80 \mathrm{N3}$ & 0.76 & $2.18 \mathrm{~N}^{4}$ & $8.15 \mathrm{~N} 3$ & $9.1 \mathrm{~N} 3$ & & & & & & \\
\hline 11 & $5.81 \mathrm{N3}$ & $4.57 \mathrm{N3}$ & 0.79 & & & & $1.12 N 6$ & & & $8.01 \mathrm{~N}$ & & \\
\hline 12 & $3.81 \mathrm{N3}$ & $2.99 \mathrm{N3}$ & 0.78 & $1.83 \mathrm{~N} / 4$ & $3.37 \mathrm{~N} 3$ & $2.46 \mathrm{~N} 3$ & & $1.57 \mathrm{~N} 3$ & $1.26 \mathrm{~N} 3$ & & $1.74 \mathbb{N} 3$ & $1.35 \mathrm{~N} 3$ \\
\hline 13 & $2.78 \mathrm{N3}$ & $2.16 \mathrm{N3}$ & 0.78 & & & & $1.12 \mathrm{~N} 6$ & & & $1.13 \mathrm{~N} 6$ & & \\
\hline 14 & $1.68 \mathrm{N3}$ & $1.29 \mathrm{N3}$ & 0.72 & & & & & & & & & \\
\hline 15 & $1.28 \mathrm{NB}$ & $1.05 \mathrm{N3}$ & 0.82 & $1.59 \mathrm{~N} 4$ & $1.09 \mathrm{N3}$ & $7.95 \mathrm{~N} 4$ & $1.12 * 6$ & & & $1.24 \mathrm{N6}$ & & \\
\hline 16 & $1.10 \mathrm{N3}$ & $8.52 \mathrm{N4}$ & 0.77 & & & & & & & & & \\
\hline 17 & $1.23 \mathrm{N3}$ & $1.03 \mathbb{N 3}$ & 0.84 & $1.65 \mathrm{~N} 4$ & $9.95 \mathrm{~N} 4$ & $7.45 \mathrm{N4}$ & $1.12 N 6$ & & & $7.20 \mathrm{N6}$ & & \\
\hline 18 & $1.35 \mathrm{NB}$ & $1.12 \mathrm{N3}$ & 0.83 & & & & & $1.11 \mathrm{~N} 3$ & $8.75 \mathrm{~N} 4$ & & $1.15 \mathrm{~N} 3$ & $9.25 \mathrm{~N} 4$ \\
\hline 19 & $1.47 \mathrm{N3}$ & $1.23 \mathrm{N3}$ & 0.84 & & & & $3.49 \mathrm{N6}$ & & & $1.54 \mathrm{~N} 6$ & & \\
\hline 20 & $1.00 \mathrm{N3}$ & $1.14 \mathrm{~N}$ & 0.81 & $1.29 \mathrm{~N}_{4}$ & $1.18 \mathrm{~N} 3$ & $8.65 \mathrm{~N} 4$ & & & & & & \\
\hline 21 & $1.53 \mathrm{N3}$ & $1.27 \mathrm{N3}$ & 0.83 & & & & $5.55 \times 6$ & & & $1.43 \mathrm{N6}$ & & \\
\hline $\begin{array}{l}22 \\
23\end{array}$ & $\begin{array}{l}1.62 \mathrm{~N} \\
2.21 \mathrm{~N}\end{array}$ & $\begin{array}{l}1.33 \mathrm{N3} \\
1.84 \mathrm{N3}\end{array}$ & $\begin{array}{l}0.82 \\
0.83\end{array}$ & & & & $4.92 \mathrm{N6}$ & & & $1.85 \mathrm{~N} 6$ & & \\
\hline 24 & $2.49 \mathrm{N3}$ & $2.05 \mathrm{~N}$ & 0.82 & 8.75 N5 & $1.97 \mathrm{~N}$ & $1.48 \mathrm{~N}$ & & $2.05 \mathrm{~N} 3$ & $1.69 \mathrm{~N} 3$ & & $2.70 \mathrm{NB}$ & $2.21 \mathrm{NB}$ \\
\hline 25 & $2.45 \mathrm{N3}$ & $2.06 \mathrm{N3}$ & 0.83 & & & & $6.16 \aleph 6$ & & & $1.05 \mathrm{N5}$ & & \\
\hline 26 & $2.81 \mathrm{N3}$ & $2.37 \sqrt{3}$ & 0.84 & $9.85 \mathrm{~N}$ & $1.27 \mathrm{N3}$ & & & & & & & \\
\hline
\end{tabular}

Each segment is $2 \mathrm{~cm}$ and is numbered from inlet.

$\mathrm{C}_{\mathrm{ND}}^{\text {Blank indicates not detected. }}$ 
Table 3.3-7. Distribution of activated corrosion products in upstream sampler, CC, diffusion tubes ( $\mu \mathrm{Ci} / \mathrm{cm}$, May 28,1974$)$

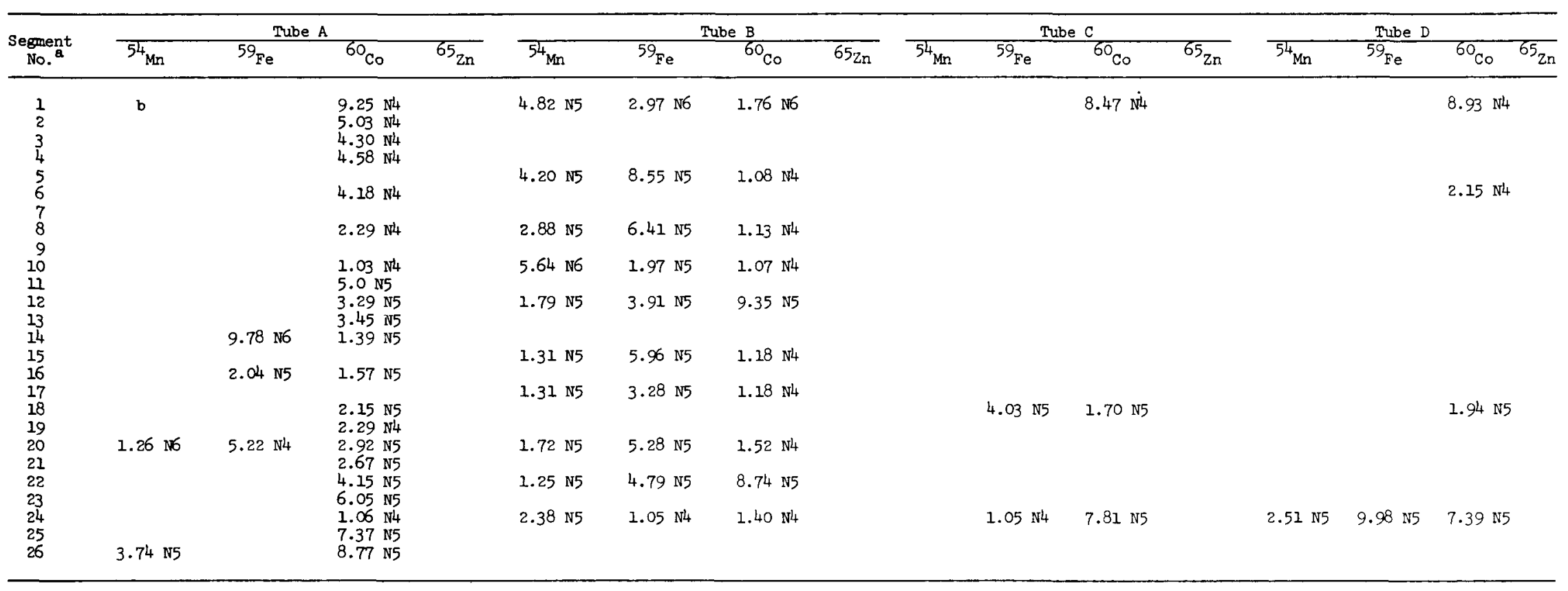

Each segment is $2 \mathrm{~cm}$ and is numbered from inlet.

Blank indicates not measured. 
Table 3.3-8. Axial distribution of nuclides in downstream sampler, CD, diffusion tubes $(\mu \mathrm{CI} / \mathrm{cm}, 28,1974)$

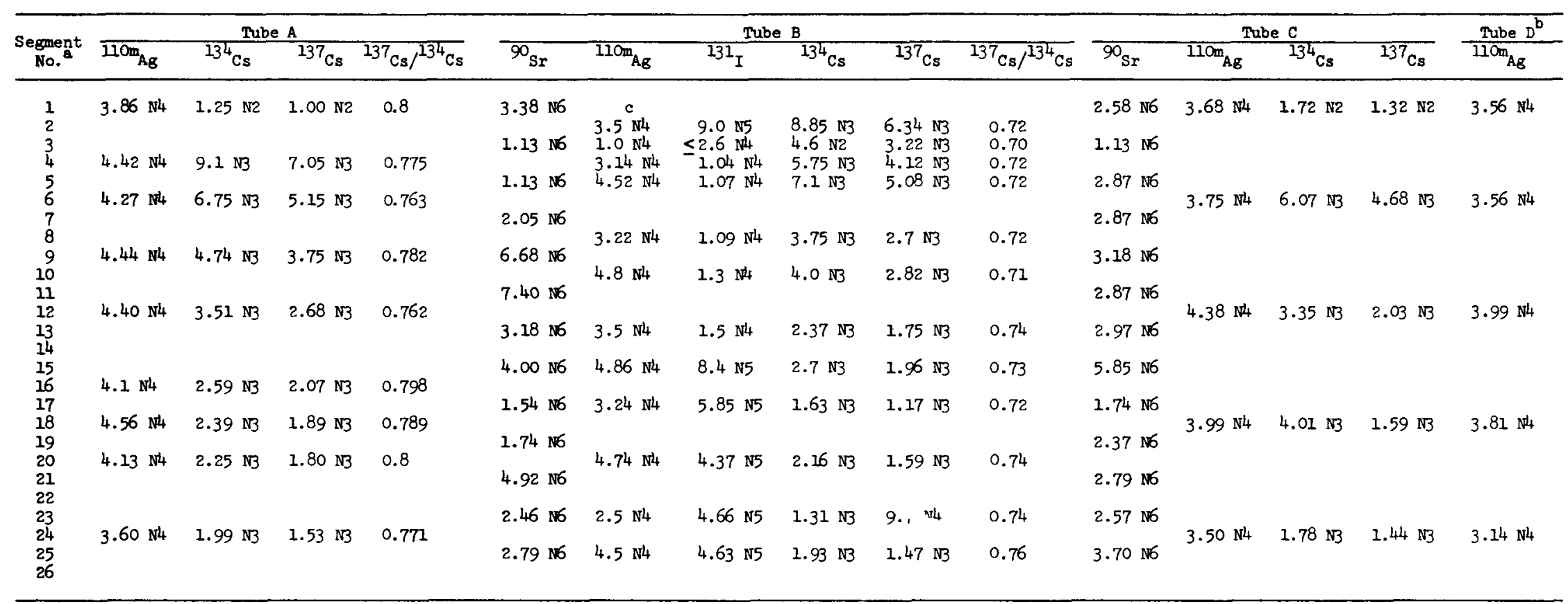

Q Each segment is $2 \mathrm{~cm}$ and is numbered from inlet.

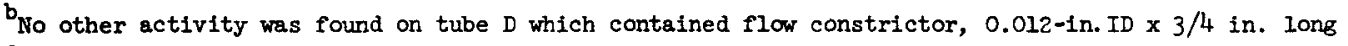

${ }^{\text {Blank indicates not measured. }}$ 
Table 3.3-9. Distribution of activated corrosion products in downstream sampler, $C D$, diffusion tubes.

$(\mu \mathrm{Ci} / \mathrm{cm}$, May 28, 1974)

\begin{tabular}{|c|c|c|c|c|c|c|}
\hline \multirow[b]{2}{*}{$\begin{array}{l}\text { Segment } \\
\text { No. }{ }^{2}\end{array}$} & \multicolumn{3}{|c|}{ Tube A } & \multicolumn{3}{|c|}{ Tube B } \\
\hline & ${ }^{54} \mathrm{Mn}$ & ${ }^{59} \mathrm{Fe}$ & ${ }^{60} \mathrm{Co}$ & ${ }^{54} \mathrm{Mn}$ & ${ }^{59} \mathrm{Fe}$ & ${ }^{60} \mathrm{Co}$ \\
\hline 1 & $N^{b}$ & ND & $2.99 \mathrm{N4}$ & & & \\
\hline 2 & $\mathrm{ND}$ & $\mathrm{ND}$ & $6.41 \mathrm{~N} 5$ & $1.01 \mathrm{~N} 5$ & $3.26 \mathrm{~N} 7$ & $1.93 \mathrm{~N} 7$ \\
\hline 3 & c & & & & & \\
\hline 4 & ND & ND & $9.32 \mathrm{~N} 5$ & $7.79 \mathrm{N6}$ & $2.26 \mathrm{N5}$ & 7.56 N5 \\
\hline 5 & ND & ND & $6.41 \mathrm{~N} 5$ & 2.02 N5 & $3.64 \mathrm{N5}$ & $1.12 \mathrm{~N} 4$ \\
\hline 6 & $N D$ & $\mathrm{ND}$ & $1.89 \mathrm{~N} 4$ & & & \\
\hline 7 & ND & $\mathrm{ND}$ & & & & \\
\hline 8 & $\mathrm{ND}$ & $\mathrm{ND}$ & & $1.04 \mathrm{~N} 5$ & 3.29 N5 & $9.19 \mathrm{N5}$ \\
\hline 9 & $1.83 \mathrm{~N} 5$ & $3.98 \mathrm{N5}$ & $1.27 \mathrm{~N} 5$ & & & \\
\hline $\begin{array}{l}10 \\
11\end{array}$ & ND & $\mathrm{ND}$ & $1.69 \mathrm{~N} 5$ & $1.38 \mathrm{~N} 5$ & $4.59 \mathrm{~N} 5$ & $1.21 \mathrm{~N} 4$ \\
\hline 12 & & & $2.03 \mathrm{~N} 4$ & & & \\
\hline 13 & $\mathrm{ND}$ & $\mathrm{ND}$ & $2.15 \mathrm{~N} 5$ & $1.46 \mathrm{~N} 5$ & $8.37 \mathrm{N5}$ & $8.81 \mathrm{N5}$ \\
\hline 14 & $3.02 \mathrm{N4}$ & ND & $6.66 \mathrm{N3}$ & & & \\
\hline 15 & $7.60 \mathrm{~N} 4$ & $N D$ & $8.06 \mathrm{N3}$ & $4.15 \mathrm{~N} 5$ & 4.89 N5 & $1.29 \mathrm{~N} 4$ \\
\hline 16 & $1.41 \mathrm{~N} 5$ & $1.06 \mathrm{~N} 4$ & 5.02 N5 & & & \\
\hline $\begin{array}{l}17 \\
18\end{array}$ & & $\begin{array}{c}\text { ND } \\
4.68 \text { N5 }\end{array}$ & $\begin{array}{l}3.48 \mathrm{~N} 5 \\
1.33 \mathrm{~N} 4\end{array}$ & $1.31 \mathrm{~N} 5$ & 4.46 N5 & $1.26 \mathrm{~N} 4$ \\
\hline 19 & & ND & 3.38 N5 & & & \\
\hline 20 & $1.95 \mathrm{~N} 5$ & 5.62 N5 & $1.86 \mathrm{N5}$ & $2.23 \mathrm{~N} 5$ & $6.06 \mathrm{N5}$ & $1.25 \mathrm{~N} 4$ \\
\hline 21 & & $\mathrm{ND}$ & $3.33 \mathrm{N5}$ & & & \\
\hline 22 & $1.98 \mathrm{~N} 5$ & ND & $2.44 \mathrm{~N} 5$ & & & \\
\hline 23 & & ND & $2.54 \mathrm{~N} 5$ & $1.51 \mathrm{~N} 5$ & 3.01 N5 & 8.66 N5 \\
\hline 24 & & ND & & & & \\
\hline $\begin{array}{l}25 \\
26\end{array}$ & & $2.30 \mathrm{~N} 4$ & & $2.05 \mathrm{~N} 5$ & 7.51 N5 & $1.22 \mathrm{~N} 4$ \\
\hline 27 & & & $3.93 \mathrm{~N} 5$ & & & \\
\hline
\end{tabular}

${ }_{b}^{a}$ Each segment is $2 \mathrm{~cm}$ and is numbered from inlet.

$\mathrm{b}$ indicates not detected.

Blank indicates not measured. 
Table 3.3-10. Axial distribution of ${ }^{134} \mathrm{Cs}$ and ${ }^{137} \mathrm{Cs}$ in sampler nozzles $(\mu \mathrm{Ci} / \text { segment })^{\mathrm{a}}$

\begin{tabular}{|c|c|c|c|c|c|c|c|c|}
\hline \multirow{2}{*}{$\begin{array}{l}\text { Segment } \\
\text { No. }\end{array}$} & \multicolumn{2}{|c|}{ CA nozzle } & \multicolumn{2}{|c|}{ CB nozzle } & \multicolumn{2}{|c|}{ CC nozzle } & \multicolumn{2}{|c|}{$C D$ nozzle } \\
\hline & $\overline{134 \mathrm{Cs}^{\mathrm{b}}}$ & $137 \mathrm{Cs}^{\mathrm{b}}$ & $\overline{134} \mathrm{Cs}^{\mathrm{b}}$ & $137 \mathrm{Cs}^{\mathrm{b}}$ & $\overline{134 \mathrm{Cs}^{\mathrm{c}}}$ & $137 \mathrm{Cs}^{c}$ & $\overline{1} 3{ }^{4} \mathrm{Cs}^{\mathrm{c}}$ & $137 \mathrm{Cs}^{\mathrm{C}}$ \\
\hline 1 & 1. $2 E-2$ & $9.6 E-3$ & $7.2 E-3$ & $7 \cdot 3 \mathrm{E}-3$ & 0.35 & 0.22 & 0.28 & 0.18 \\
\hline 2 & $d$ & & $3 \cdot 7 E-3$ & $3 \cdot 8 \mathrm{E}-3$ & 0.11 & 0.067 & 0.22 & 0.14 \\
\hline 3 & & & $3 \cdot I E-3$ & $4 . \mathrm{OE}-3$ & 0.13 & 0.084 & 0.16 & 0.10 \\
\hline 4 & $9 \cdot 3 E-3$ & $7 \cdot 3 E-3$ & $3 \cdot 3 E-3$ & $3 \cdot 3 E-3$ & 0.10 & 0.065 & 0.12 & 0.078 \\
\hline 5 & $7 \cdot 9 \mathrm{E}-3$ & $6.7 \mathrm{E}-3$ & $2.9 \mathrm{E}-3$ & $3 . \mathrm{OE}-3$ & 0.052 & 0.033 & 0.091 & 0.059 \\
\hline
\end{tabular}

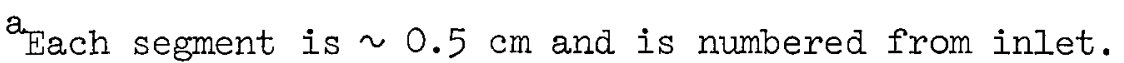

bactivities corrected for decay to May 18, 1973.

${ }^{c}$ Activities corrected for decay to May 28, 1974.

$\mathrm{d}_{\text {Blank indicates not measured. }}$ 


\subsection{Discussion of Diffusion Tube Results}

\section{4-1 Theoretical behavior of diffusion tubes}

The axial distribution of dust concentration in the diffusion tube is given by the so-called Gormley-Kennedy ${ }^{7}$ equation,

$$
\frac{n(z)}{n_{0}}=0.819 e^{-3.657 d}+0.097 e^{-22.3 d}+0.0032 e^{-57 d}+\ldots,
$$

where

$$
\begin{aligned}
\mathrm{n}(\mathrm{z}) & =\text { concentration at axial location } \mathrm{z}, \mathrm{mole} / \mathrm{cm}^{3}, \\
\mathrm{n}_{0} & =\text { concentration at inlet, } \\
d & =\pi D \mathrm{D} / \mathrm{Q}, \\
D & =\text { diffusion coefficient }, \mathrm{cm}^{2} / \mathrm{sec}, \\
Q & =\text { volumetric flow, } \mathrm{cm}^{3} / \mathrm{sec},
\end{aligned}
$$

under the following conditions: ( 1 ) the flow in a tube is fully developed and laminar, (2) the wall of the tube acts as an infinite sink for some material convected along with the carrier helium, (3) the concentrations are small so that axial variations, as a result of deposition, do not significantly alter the velocity, and (4) molecular diffusion alone affects the transport of this material. The rate of deposition, as a function of axial location, may be obtained by multiplying the derivative, $\mathrm{dn}(\mathrm{z}) / \mathrm{dz}$, by the volume flow rate, $Q$. The purpose of the diffusion tube was to utilize deposition profiles to distinghish between a number of different vehicles for transporting a particular nuclide. For example, in the case illustrated by Browning ${ }^{8}$ in Fig. 3.4-1, the total axial nuclide profile is shown to consist of the sum of a molecular component, characterized by a diffusion coefficient of $\sim 0.05 \mathrm{~cm}^{2} / \mathrm{sec}$, and two particulates that measure $0.01 \mu \mathrm{m}$ and $0.3 \mu \mathrm{m}$ in size, characterized by much smaller diffusion coefficients. The figure illustrates how the total profile may be analyzed for the various species contributing to the total transport rate.

Browning's work demonstrated that, under carefully controlled conditions, deposition profiles of molecules in a diffusion tube can be accurately described by the Gormley-Kennedy equation. Further, Browning 


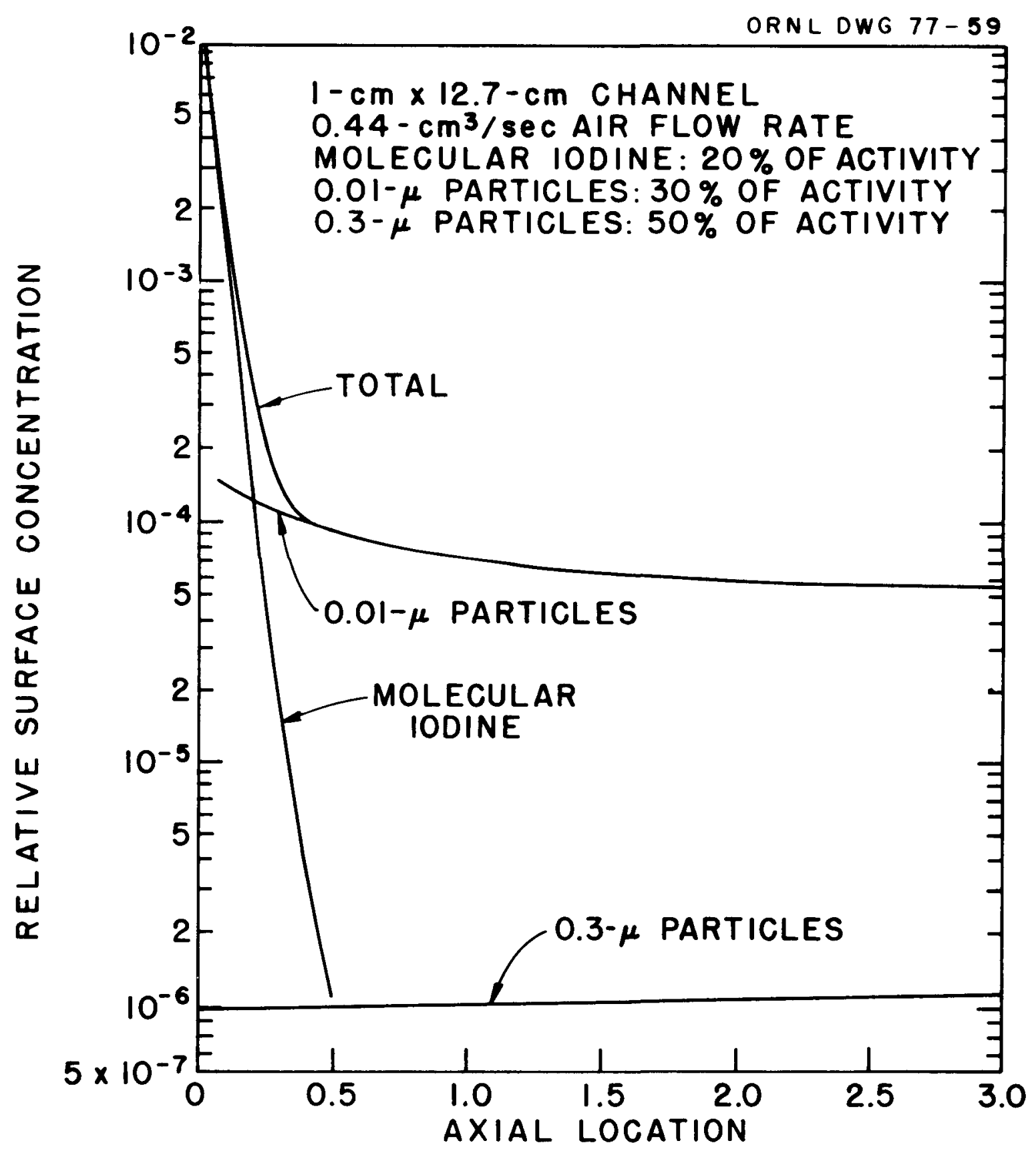

Fig. 3.4-1. Theoretical diffusional deposition of various species in a rectangular diffusion channel. ${ }^{8}$ 
showed that certain aerosols deposit as predicted, employing the lower values for diffusion coefficient appropriate for particles. Apparently "correct" diffusion tube profiles were obtained for uniformly sized alumina aerosols of $0.005-\mu \mathrm{m}$ diameter, fog droplets of $0.007 \mu \mathrm{m}$, and smoke composed of $0.25-\mu m$-diam droplets.

Unfortunately, the diffusion tubes in the coolant samplers operated under more complex circumstances than would exist under laboratory tests of the device. One obvious complicating factor is the probable existence of a wide spectrum of particulate sizes instead of the controlled monodispersions generated in laboratory tests. Thus, there may exist a gradual progression of effective diffusion coefficients ranging from the molecular value down to zero.

A second potentially complicating factor is the possible existence of significant radial temperature gradients within the flowing gases of the coolant-sampler diffusion tubes in a direction that would serve to increase the effective diffusion coefficient. Schwendiman ${ }^{9}$ shows that gradients of $100^{\circ} \mathrm{C} / \mathrm{cm}$ may effect the thermal diffusion rate sufficiently to overcome the gravitational force on particles less than $0.6 \mu \mathrm{m}$ (assuming a density of $2.3 \mathrm{~g} / \mathrm{cm}^{3}$ ). Hence, the radial temperature gradient in the diffusion tubes employed in these experiments may have had a significant effect on observed axial distributions at certain locations.

\subsubsection{Effect of the nozzle on coolant sampler performance}

Since the nozzle openings to the flow were merely 0.031 in. and $0.041 \mathrm{in.}(787 \mu \mathrm{m}$ and $104 \mathrm{I} \mu \mathrm{m})$ for the upstream and downstream samplers, respectively, one may question the degree of accuracy of the sampling procedure even assuming accurate, isokinetic operation of the probe. For example, in sampling tests performed by Griffiths ${ }^{10}$ on coal dust particles of mean size $6 \mu \mathrm{m}$, airborne in a 12-in.-diam conduit, it was found that highly erroneous results were obtained with a 1/4-in. sampler even when isokinetic conditions were maintained, whereas accurate results were obtained employing a 1-in.-diam sampler. In other words, accurate isokinetic samples were obtained when the sampler size exceeded $1700 d_{p}$ or 
$0.07 d_{t}$, where $d_{p}$ and $d_{t}$ are the particle size and conduit diameter, respectively.

Assuming the first criterion to be valid, the coolant samplers would be grossly inaccurate for particle sizes exceeding $\sim 0.5 \mu$. The second criterion indicates that samplers of $\sim 1.7-i n$. diameters would have been required to yield accurate isokinetic samples of the convected particulates of a size greater than or equal to $5 \mu \mathrm{m}$.

It is interesting to consider the degree to which deposition in the nozzle affected sampler results. Table 3.2-2 shows that for the $\mathrm{Cl}$ upstream sampler, more than 50\% of the deposited Cs in the nozzle/ diffusion tube regions was on the nozzle. The ratios are much higher for the activated corrosion products, exceeding $90 \%$ for ${ }^{54} \mathrm{Mn}$. The smallest observed ratio was 0.39 for ${ }^{110 \mathrm{~m}} \mathrm{Ag}$. Similarly, almost equal amounts of Cs were deposited in the nozzle as in all the diffusion tubes, for the CB, $\mathrm{CC}$, and $\mathrm{CD}$ samplers.

Assuming the Gromley-Kennedy equation can be applied to the nozzle as well as to the diffusion tubes, one can show that for a molecular diffusion coefficient of $0.06 \mathrm{~cm}^{2} / \mathrm{sec}$, typical for the temperature regime of the downstream sampler, the deposition rate in the nozzle should be equal to $\sim 17 \%$ of the sum of the rates of all the diffusion tubes. For the upstream samplers, where the temperatures are higher, a greater portion of the molecular activity is predicted to be deposited in the nozzle portion of the sampler. For example, atomic I may have a diffusion coefficient of $\sim 0.13 \mathrm{~cm}^{2} / \mathrm{sec}$ under upstream sampler conditions, in which case, the Gormley-Kennedy equations predict that $\sim 67 \%$ of the total nozzle/ diffusion tube activity will be deposited in the nozzle. For particulates, on the other hand, the Gormley-Kennedy equation reduces to:

$$
\text { deposition rate } \frac{\text { nozzle }}{\text { diffusion tubes }}=\frac{I_{n}}{L_{d t}} \text {, }
$$

where $I_{n}$ and $I_{d t}$ are the respective lengths of the nozzle portions and the diffusion tubes. Hence, for particulates, one would predict a smaller nozzle deposition of $\sim 5 \%$ of the diffusion tubes value. 
However, the validity of the Gormley-Kennedy equation within the nozzle is questionable because the flow there has not had an opportunity to become fully developed. For the upstream samplers CA and CC, a Reynolds number of 1250 is calculated for the nozzle, indicating a tendency for laminar flow, but an $\mathrm{L} / \mathrm{D}$ of $\sim 50$ is required for the full establishment of the flow profile. Hence, the flow pattern in the nozzle is developing from flat to parabolic, a process which tends to increase radial transport. In addition, the $90^{\circ}$ bend in the nozzle would cause increased deposition above that predicted from the Gormley-Kennedy equation.

\subsubsection{Axial distribution of nuclides on the sampler diffusion tubes}

Figures 3.4-2 through 3.4-7 illustrate some of the axial profiles observed on diffusion tubes in samplers $\mathrm{Cl}, \mathrm{CA}, \mathrm{CB}, \mathrm{CC}$, and $\mathrm{CD}$. The

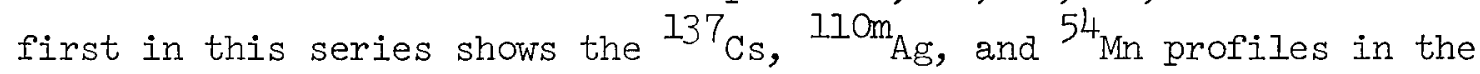
Cl downstream sampler. As the discussion in Sect. 3.4.1 has shown, the generally flat profile for these nuclides indicates transport via dust particles carried along with the flow. In contrast, the profiles shown in Fig. 3.4-3 for $\mathrm{Cs}$ in the $\mathrm{CA}$ upstream sampler indicate substantial transport as molecular Cs. This is indicated by the steep slopes for ${ }^{134} \mathrm{Cs}$ and ${ }^{137} \mathrm{Cs}$ up to $28 \mathrm{~cm}$ from the inlet of the diffusion tube. On the other hand, iodine seems to have been carried principally by particulates.

Figures 3.4-4 and 3.4-5 pertain to diffusion tubes $A$ and $C$ of the downstream sampler $C B$ removed at the same time as upstream sampler $C A$. Note that in both cases, the Cs profiles are similar but flatter than seen in the concurrent downstream sampler, which may indicate increased dust levels downstream from the steam generator. These figures also indicate the ${ }^{13} I_{I}$ profile to be rather atypical for a diffusion tube and the ${ }^{110 m_{A g}}$ profile to be of almost zero slope.

Figures 3.4-6 and 3.4-7 illustrate the observed profiles for Cs in the final pair of coolant samplers, the upstream CC and downstream CD samplers. An ${ }^{13 I_{I}}$ profile is shown for the upstream CC sampler, and a $110 m_{\mathrm{Ag}}$ profile is shown for the downstream $C D$ sampler. 


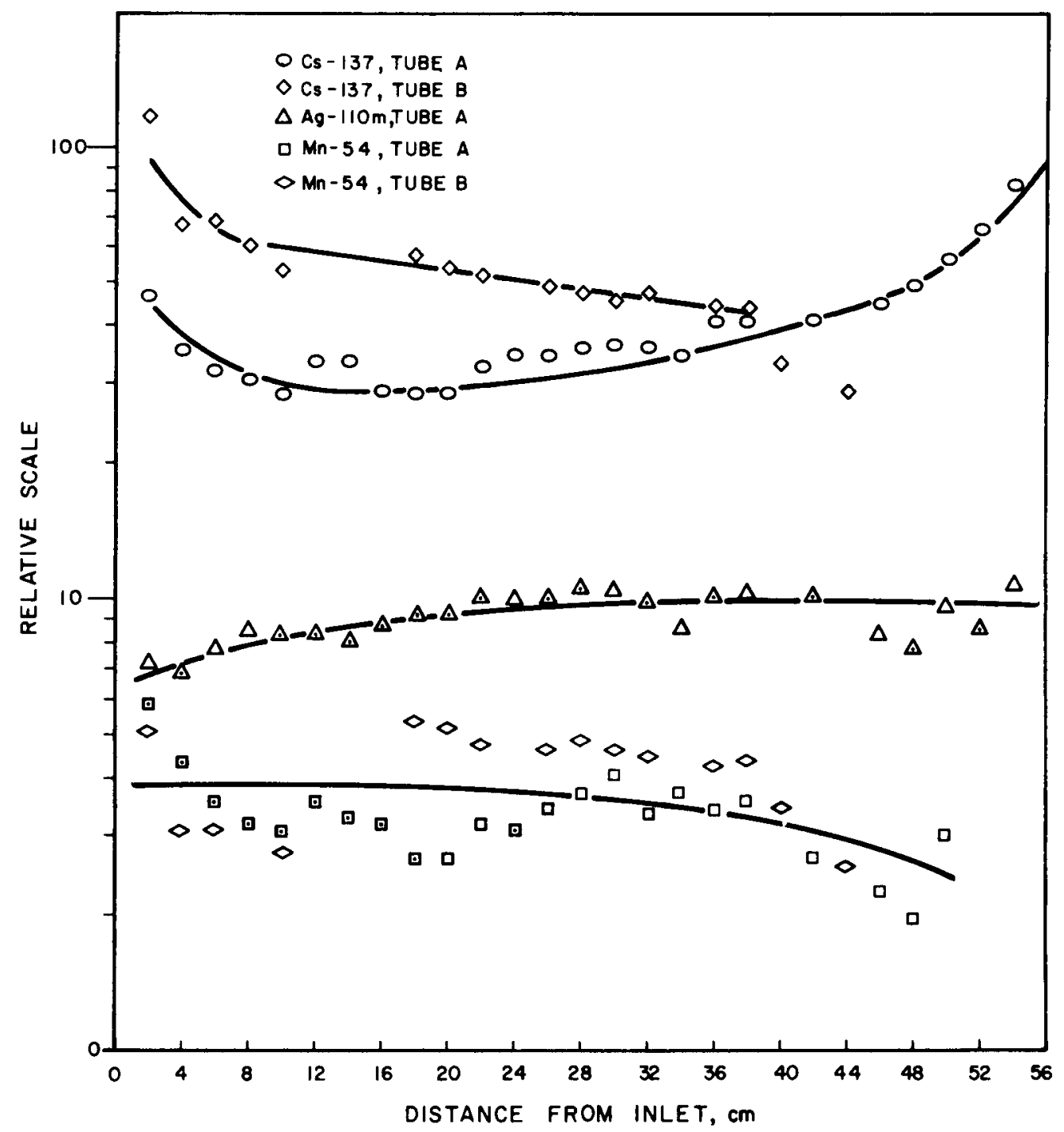

Fig. 3.4-2. Distribution of ${ }^{54} \mathrm{Mn},{ }^{110 \mathrm{~m}} \mathrm{Ag}$, and ${ }^{137} \mathrm{Cs}$ in downstream sampler Cl diffusion tubes, January 8, 1972. 


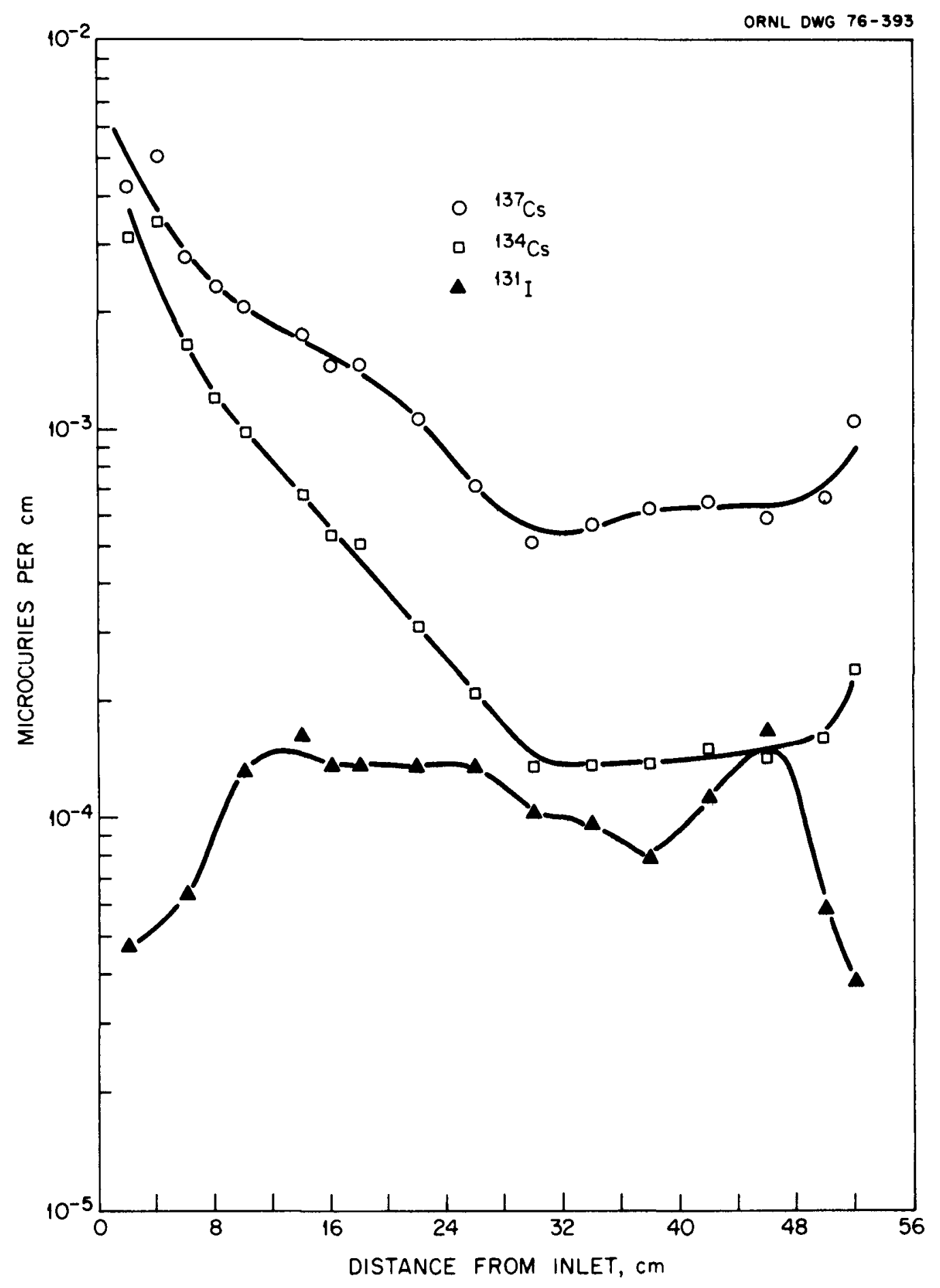

Fig. 3.4-3. Distribution of radionuclides on diffusion tube A of upstream sampler, CA, May 18, 1973. 


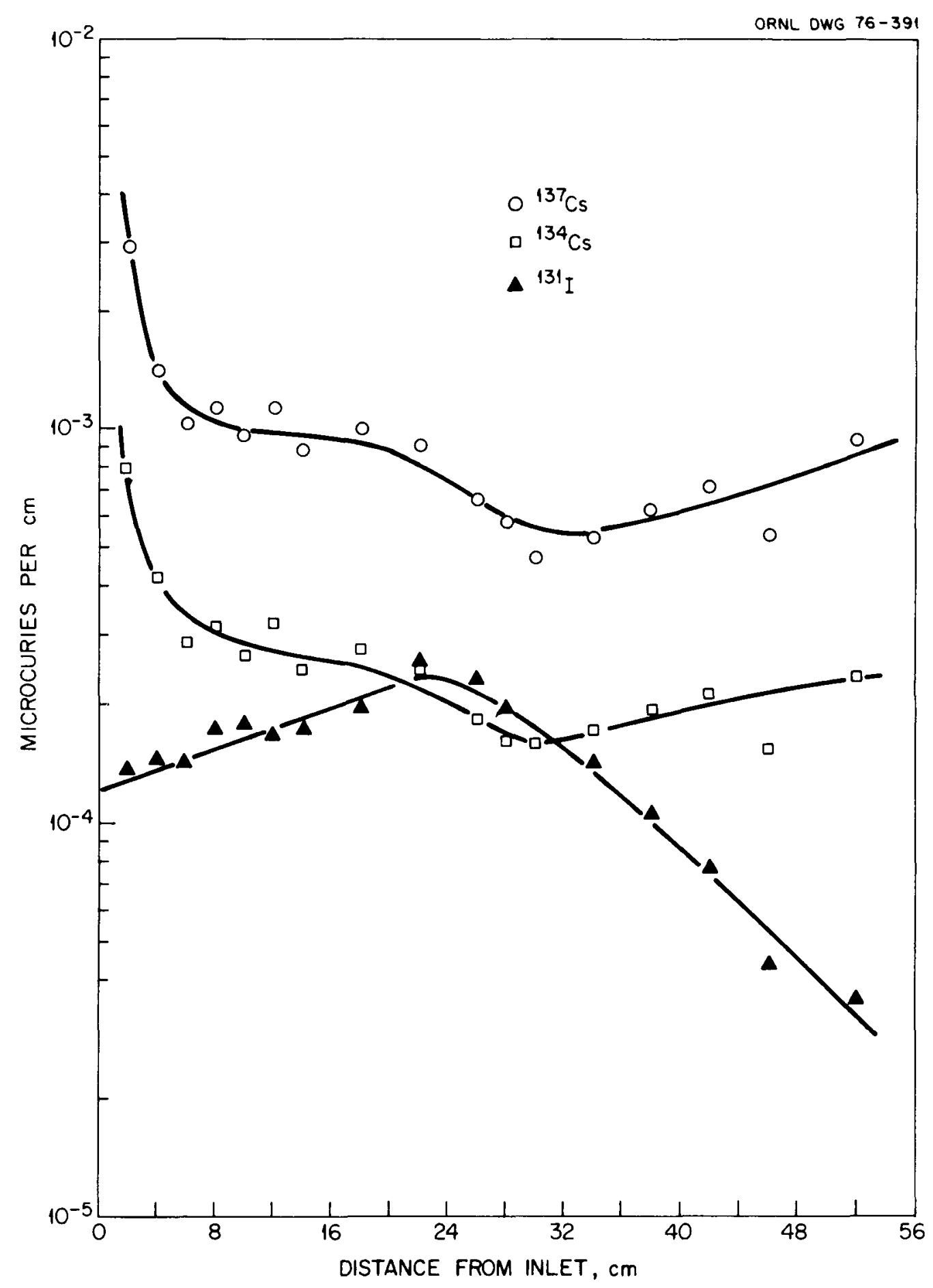

Fig. 3.4-4. Distribution of radionuclides on diffusion tube $A$ of downstream sampler, CB, May 18, 1973. 


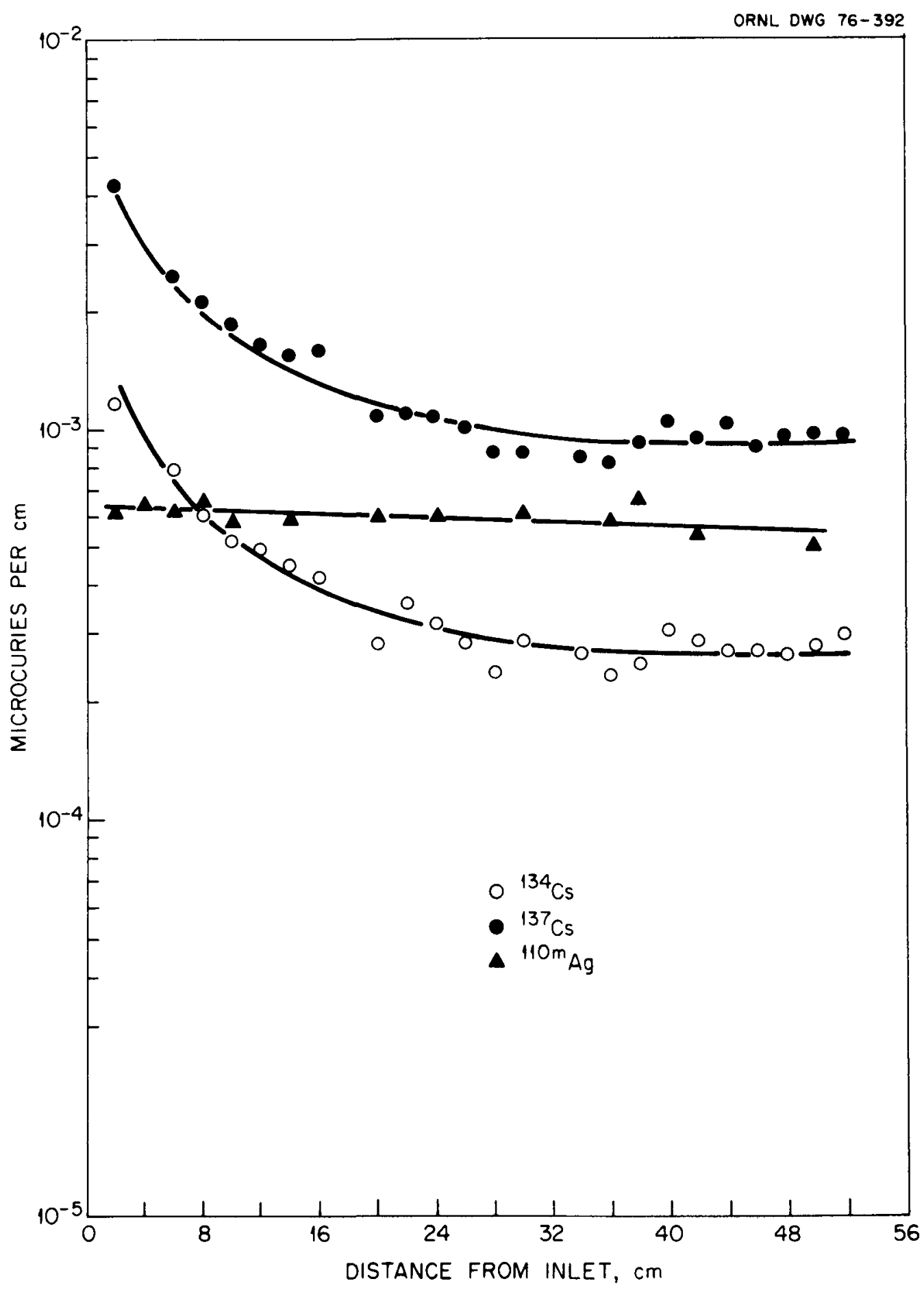

Fig. 3.4-5. Distribution of ${ }^{110 \mathrm{O}} \mathrm{Ag},{ }^{134} \mathrm{Cs}$, and ${ }^{137} \mathrm{Cs}$ on diffusion tube C of downstream samplex, CB, May 18, 1973. 


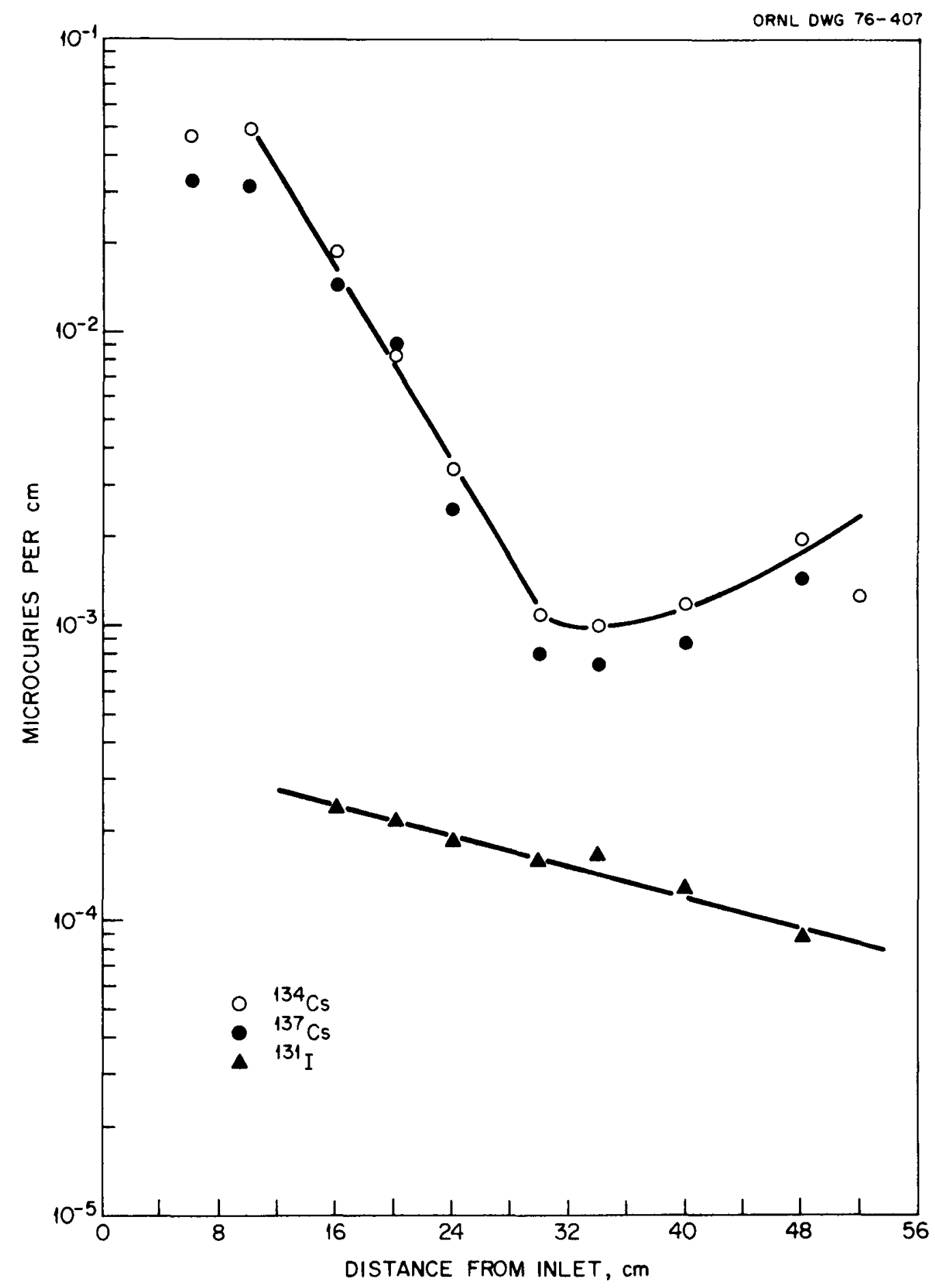

Fig. 3.4-6. Distribution of ${ }^{131} \mathrm{I},{ }^{134} \mathrm{Cs}$, and ${ }^{137} \mathrm{Cs}$ on diffusion tube B of upstream sampler, CC, May 28, 1974. 


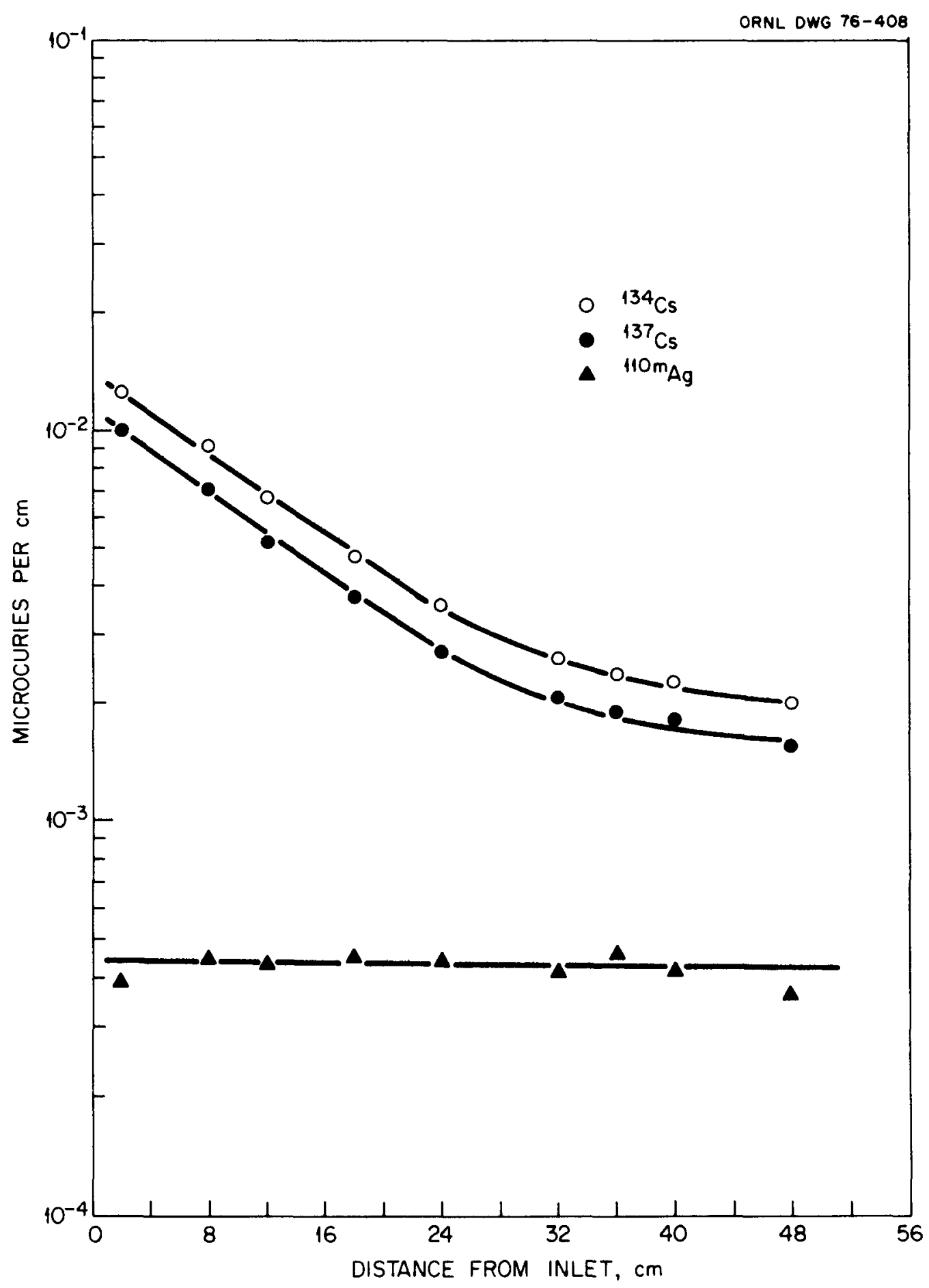

Fig. 3.4-7. Distribution of ${ }^{110 \mathrm{~m}} \mathrm{Ag},{ }^{134} \mathrm{Cs}$, and ${ }^{137} \mathrm{Cs}$ in diffusion tube A of downstream sampler, CD, May 28, 1974. 
Although interpretation of these profiles requires some careful analysis, some general phenomena seem evident. If one views the sequence of $\mathrm{Cs}$ profiles for the downstream samplers $\mathrm{Cl}, \mathrm{CB}$, and $\mathrm{CD}$, a diminishing degree of deposition due to dust is apparent--an observation that is in accord with primary circuit gamma-scan data and the dust sampler data. Also, it is observed that iodine and silver profiles are usually quite flat.

\section{References for Section 3}

1. R. A. Lorenz, "Design of Impactor for the Peach Bottom Sampler," Intra. Lab. corresp., Oct. 7,.1971.

2. J. O. Kolb, "Design Description and QA Program Plan for Peach Bottom HTGR Sampling Systems," Internal memorandum (June 4, 1971).

3. Peach Bottom Atomic Power Station, Semi-Annual Operations Report No. 67, Period July 1 to Dec. 31, 1972.

4. Peach Bottom Atomic Power Station, Semi-Annual Operations Report No. 69, Period July 1 to Dec. 30, 1973.

5. L. D. Hulett, H. W. Dunn, Intra-lab letter to H. J. de Nordwall, "Study of Dust Samples CBD-204 to CBD-504 from Peach Bottom," Nov. 5, 1973.

6. I. D. Hulett, intra-lab letter to R. P. Wichner, "Study of Dust Samples CD2 to CD5, CA2F, CBF from Peach Bottom," June 26, 1976.

7. P. G. Gormley and M. Kennedy, "Diffusion from a stream Flowing Through a Cylindrical Tube," Proc. of the Roy. Irish Acad., 52-A (1949), pp. $163-169$.

8. W. E. Browning and R. D. Ackely, "Characterization of Millimicron Aerosols and Their Removal from Gases," The 3rd Conf. on Nuclear Chem., Gatlinburg (1962), pp. 130-147.

9. I. C. Schwendiman et al., "Radioactive Particle Retention in Aerosol Transport Systems," in Radioactive Pollution of Gaseous Media (Interm. Colloq.), Saday 12-16 (1963), pp. 373-385.

10. J. H. Griffiths, "Determination of Dust Concentrations in Mine Atmospheres," Trans. Inst. Min. Eng. 99, 150 (1940). 


\section{FISSION GAS CONCENTRATIONS IN THE PRIMARY COOIANT AND CORE PURGE GAS}

\subsection{General Description of Fission Gas Experiment}

Measurements of fission gases ( $\mathrm{Kr}$ and $\mathrm{Xe}$ isotopes) we re made on three occasions during the operation of Core 2--Dec. 5, 1970, Feb. 19, 1973, and sept. 11 through 14, 1973. The primary coolant was sampled on each occasion, but the core purge gas was measured only in the last experiment. These experiments were conducted by ORNL in conjuction with General Atomic Company (GAC). Additional measurements were performed by the Philadelphia Electric Company (PEC) and GAC utilizing somewhat different procedures. ${ }^{1}$ A principal motive in these experiments was to measure the fission gases $90_{\mathrm{Kr}}$ and $137 \mathrm{Xe}$ in the primary circuit and thus enable estimates to be made of the amounts of ${ }^{90} \mathrm{Sr}$ and ${ }^{137} \mathrm{Cs}$ that enter the primary circuit via their gaseous parents.

On each occasion, different measurement procedures were used, which are described in more detail in sect. 4.2. The December 1970 experiment was of a preliminary nature to determine anticipated fission gas levels and to help plan for further experimentation. At that time, the gammadetector was set up adjacent to the accessible "hot-valve cooling line" (HVCL). Although the continuous flow of coolant in the line had an ideally short transit time of $\sim 5 \mathrm{sec}$ from the primary system to detector location, the background radiation, principally ${ }^{60} \mathrm{Co},{ }^{134} \mathrm{Cs}$, and ${ }^{137} \mathrm{Cs}$, which accumulated in the line throughout its life, masked the signals from many of the fission gases of interest.

The second and third fission gas experiments employed the reactorradiochemical-sampling system for sample acquisition. This system, shown schematically in Fig. 4.I-I, had access to the fuel-element purge outlet flow via valve HSVI and the primary coolant of the steam generator exit via valves HSV7 and HSV8. In addition, an important feature was access to purified helium, via HSV10, used for purging the lines prior to sample acquisition. The facility could accomodate either flow-through samples or "grab" samples, and both were used. 


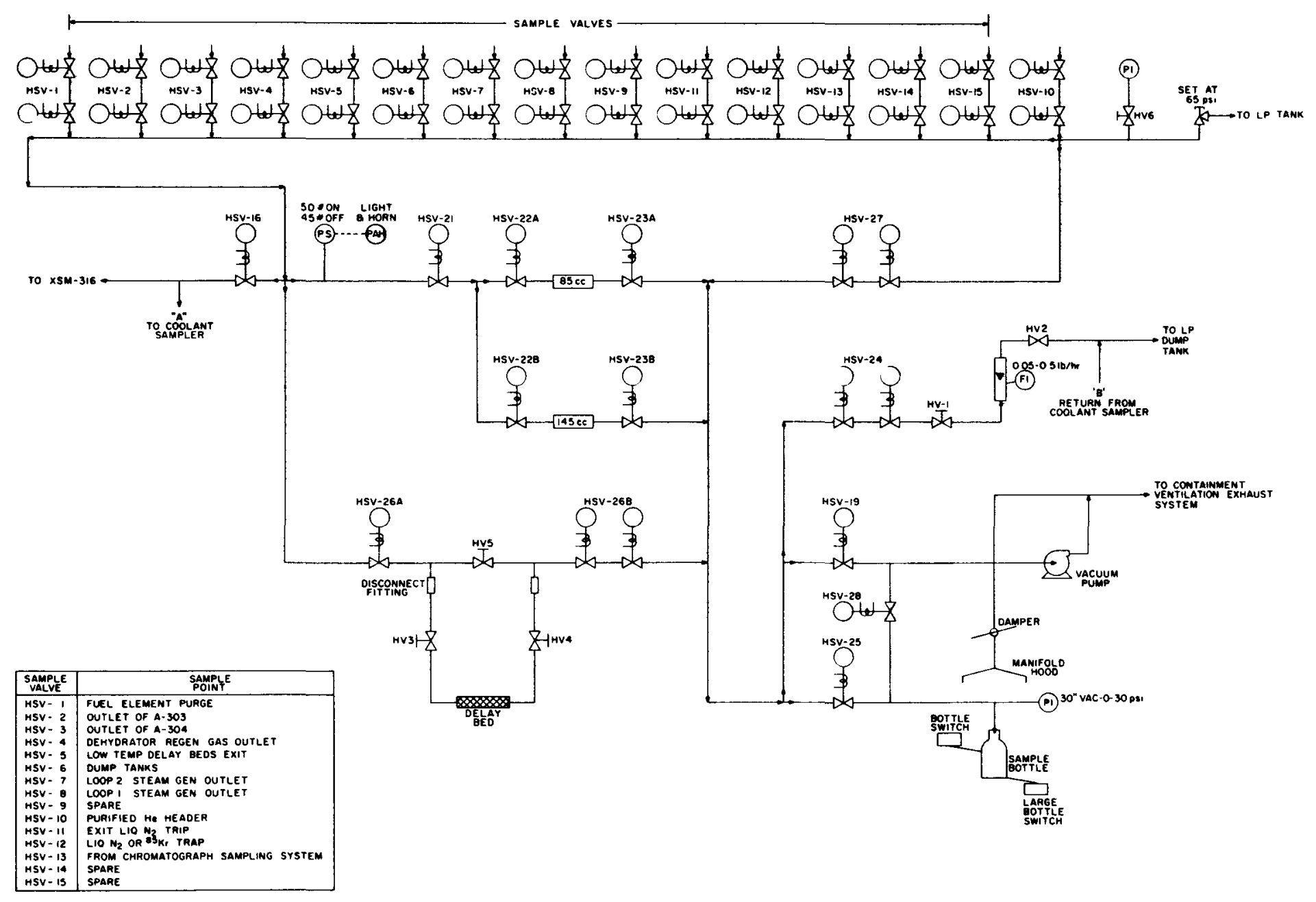

Fig. 4.1-1. Schematic of Peach Bottom radiochemical sampling station. 
For the February 1973 experiment, access to primary coolant at the Ioop-I steam-generator exit was attained using valve HSV8. A flow-through apparatus for the adsorption of $K r$ and $X e$, which contained a charcoal trap cooled by either liquid nitrogen or dry ice, was hooked into the gas sampling system at the indicated locations. The gases were collected for 6 to 20 min before gamma scanning the cell.

In the third test series conducted in sept. 1973, three types of procedures were used:

1. Primary coolant was adsorbed on charcoal in the same fashion as the February experiments except with larger collection times on the order of $2 \mathrm{hr}$.

2. Fission gas activity was determined in the primary coolant utilizing a flow-through cell hooked into the gas sampling systems at the same location as the charcoal trap.

3. Grab samples of fuel-element purge gas were obtained utilizing the container termed "sample bottle" in Fig. 4.1-I.

Operational procedures for obtaining flow-through or grab samples are outlined in ref. 2 .

\subsection{Experimental Methods}

\subsubsection{December 1970 experiment}

The experiment of Dec. 1970 was of a scouting nature to ascertain if fission gases in the primary coolant might be measured in situ. A gamma spectrum was acquired with a $\mathrm{Ge}(\mathrm{Li})$ detector placed adjacent to a pipe through which the primary circuit coolant circulated. This pipe, the hotvalve cooling line (HVCL), conducted helium from the loop-l main heliumcompressor outlet to the loop-l hot-duct (concentric duct) block valve. The coolant served to prevent overheating of certain parts of the block valve. The pipe was routed to a flow controller in the air room, and it was at this location that a section of $\sim 2$ ft was accessible for counting. The HVCL consisted of nominal 1.5-in.-diam sched 80 pipe. Helium circulated through the pipe at a rate of $\sim 115 \mathrm{Ib} / \mathrm{hr}$, with a temperature and pressure 
of $\sim 600^{\circ} \mathrm{F}$ and $340 \mathrm{psig}$, respectively. The length of pipe from the compressor to the measurement point was $\sim 85 \mathrm{ft}$. It is estimated that $\sim 5 \mathrm{sec}$ was required for the coolant to flow from the compressor to the measurement point.

The fission gases detected in the HVCL, and their corresponding concentrations in the primary coolant in units of $\mathrm{pCi} / \mathrm{scc}$ (standard cubic centimeter), are presented in Table 4.2-1. Also given in Table 4.2-1 are the concentrations of a few fission gases independently measured by Pniladelphia Electric Co. (PEC) on Nov. 30, and Dec. 11, 1970, on samples drawn from the gas sampling stations. 3 The method used to convert net photopeak count rates to fission gas concentrations was based on the use of the ${ }^{88} \mathrm{Kr}$ concentration determinations, reported by $\mathrm{PEC}$, as an "internal standard" and the measured photopeak count rates of ${ }^{56} \mathrm{Mn}$ observed in the HVCL which emits several gamma rays of different energies. The details of this method of standardization are presented in Appendix $A$ of this report.

Table 4.2-1. Concentration of fission gases in Peach Bottom primary coolant measured in hot-valve cooling line

$$
\text { (HVCL), December 5, } 1970
$$

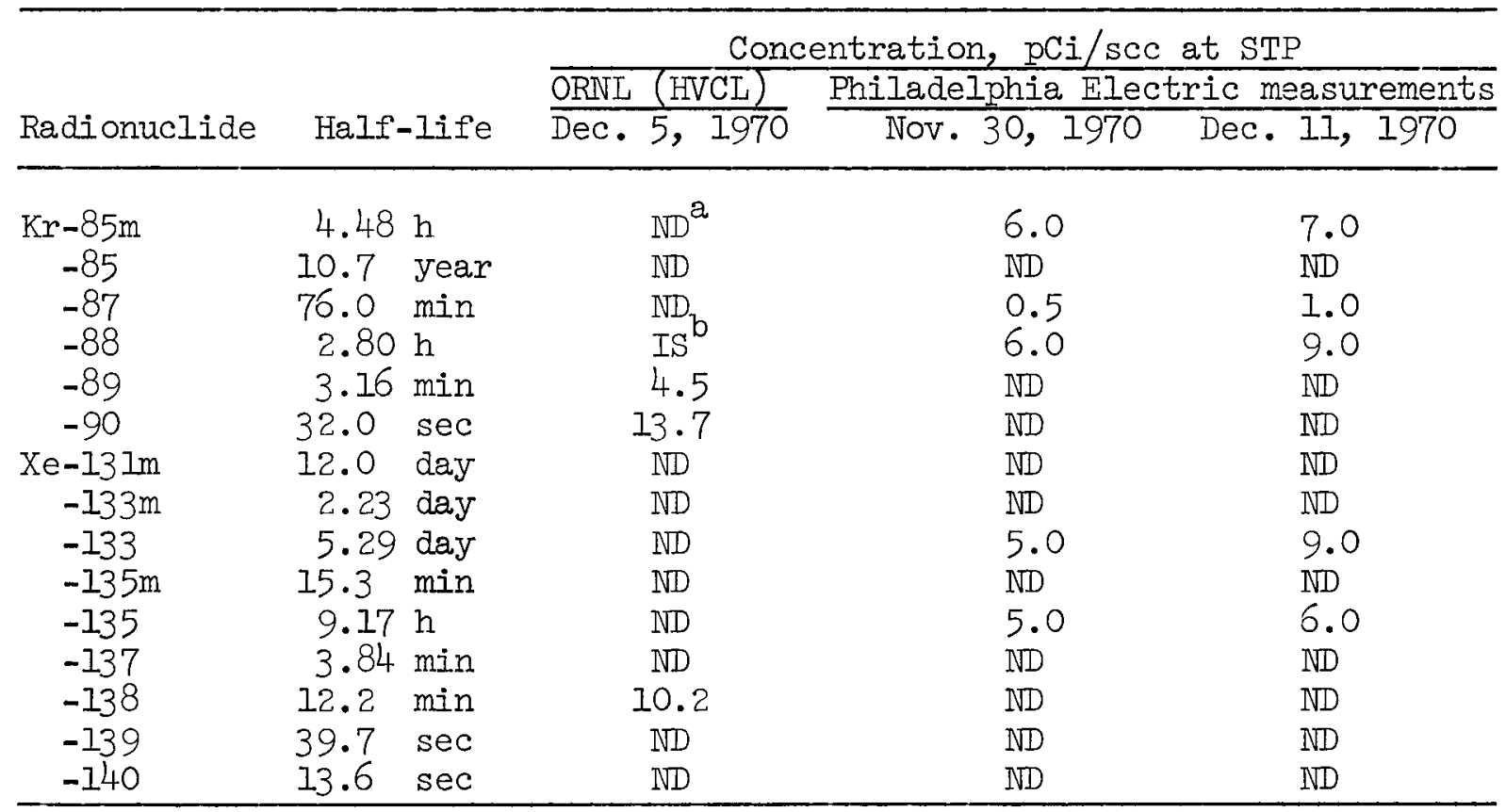

$a_{\mathrm{ND}}$ indicates fission gas was not detected.

Used as internal standard. 
Several nongaseous fission products, including fission gas daughters, and neutron capture products were also detected in the pipe, presumably as plateout activities on the pipe wall. The concentrations of these radionuclides were first computed as if they existed in the gas in units of apparent $\mathrm{pCi} / \mathrm{scc}$ and were converted to surface units using the pipe dimensions and the gas temperature and pressure. These nongaseous radionuclides were detected and their concentrations, expressed as $\mathrm{pCi} / \mathrm{cm}^{2}$ of pipe surface, are given in Table 4.2-2.

Table 4.2-2. Spacific surface concentrations of nongaseous radionuclides measured in hot-valve cooling line,

December 5, 1970

\begin{tabular}{lrr}
\hline Radionuclide & $\mathrm{pCi} / \mathrm{cm}^{2}$ & Type of radionuclide \\
\hline $\mathrm{Na}-24$ & 100 & $\mathrm{n}, \gamma$ \\
$\mathrm{Mn}-56$ & 1,100 & $\mathrm{n}, \gamma$ \\
$\mathrm{Mn}-54$ & 300 & $\mathrm{n}, \gamma$ \\
$\mathrm{Fe}-59$ & 1,600 & $\mathrm{n}, \gamma$ \\
$\mathrm{Co}-60$ & 4,600 & $\mathrm{n}, \gamma$ \\
$\mathrm{Rb}-88$ & 130 & $\mathrm{FP}$ \\
$\mathrm{Rb}-89$ & 150 & $\mathrm{FP}$ \\
$\mathrm{Rb}-90$ & 110 & $\mathrm{FP}$ \\
$\mathrm{Zr}-95$ & 690 & $\mathrm{FP}$ \\
$\mathrm{Ru}-106$ & 4,100 & $\mathrm{FP}$ \\
$\mathrm{Ag}-110 \mathrm{~m}$ & 460 & $\mathrm{FP}$ \\
$\mathrm{Cd}-115 \mathrm{~m}$ & 790 & $\mathrm{FP}$ \\
$\mathrm{In}-115$ & 1,200 & $\mathrm{FP}$ \\
$\mathrm{Cs}-134$ & 7,800 & $\mathrm{FP}$ \\
$\mathrm{Cs}-137$ & 13,000 & $\mathrm{FP}$ \\
$\mathrm{Cs}-138$ & 280 & $\mathrm{FP}$ \\
$\mathrm{La}-140$ & 120 & $\mathrm{FP}$ \\
$\mathrm{Ce}-144$ & 18,000 & $\mathrm{FP}$ \\
\hline
\end{tabular}

$a_{n, \gamma}$ denotes neutron capture product; FP denotes fission product.

As can be seen in Table 4.2-1, the only fission gases detected in the hot-valve cooling line were ${ }^{87} \mathrm{Kr},{ }^{88} \mathrm{Kr},{ }^{89} \mathrm{Kr},{ }^{90} \mathrm{Kr}$, and ${ }^{138} \mathrm{Xe}$. The relatively large amounts of ${ }^{60} \mathrm{Co},{ }^{134} \mathrm{Cs}$, and ${ }^{137} \mathrm{Cs}$ present in the pipe produced large photopeaks and compton distributions in the gamma spectra thus swamping the photopeaks of low intensity gamma rays with energies below 


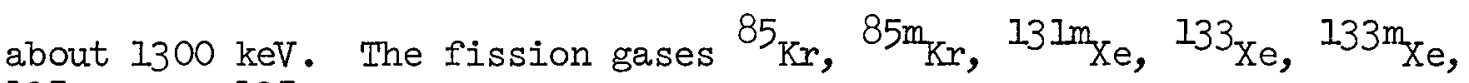

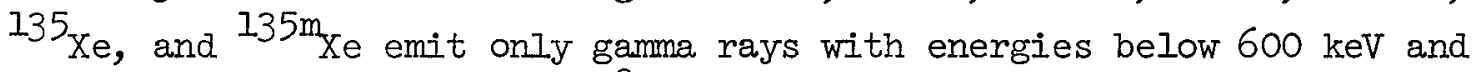
were not observed. Although $87 \mathrm{Kr}$ has only one major gamma ray with an energy of $402 \mathrm{keV}$, it was barely detectable by means of two minor gamma rays, whose energies are 2554 and $2558 \mathrm{keV}$. Xenon-137 has a major gamma ray with an energy of $455 \mathrm{keV}$ and several minor gamma rays above $1300 \mathrm{keV}$. The branching ratios of these higher-energy gamma rays are very small, however, and no photopeaks of ${ }^{137}$ Xe were found. Xenon-139 emits no gamma rays with energies above $800 \mathrm{keV}$, and none of its photopeaks were observed. A very small photopeak was found with an energy of $\sim 1413 \mathrm{keV}$ which corresponded to a major garma ray of ${ }^{140} \mathrm{Xe}$. However, none of the other gamma rays of ${ }^{140} \mathrm{Xe}$, all of which have lower energies, were observed, and, thus, the detection of ${ }^{140}$ Xe was judged to be too uncertain to warrant the calculation of its concentration.

Initially, it was thought that the HVCL might offer a means to easily and rapidly measure the fission gases in the coolant; however, several factors prevented its effective use. Because the temperature of the pipe was rather high, it was necessary to place the detector $\sim 12$ in. from the pipe to prevent thermal damage. Although a relatively large amount of coolant was accessible (over $650 \mathrm{scc}$ of coolant per inch of pipe), the large source-to-detector distance prevented efficient counting of the coolant, and the measurement of those fission gases that were detected required $\sim 10 \mathrm{hr}$ of counting time. The major difficulty in this use of the HVCL, however, was the presence of large amounts of nongaseous plateout activities. This difficulty could only have been prevented by the installation of a clean section of piping prior to a measurement or by the installation of additional piping with valves that would provide a coolant flow only during fission gas measurements.

\subsubsection{February 1973 measurements}

Fission gases in the primary coolant were measured Feb. 19, 1973, by collecting adsorbed samples on an activated charcoal bed cooled with dry ice and liquid nitrogen. The charcoal bed contained in a cell especially prepared by GAC for this purpose is shown schematically in 
Fig. 4.2-1. The charcoal bed contained in the bottom of the outlet tube of the cell had a $0.375-i n$. OD and was 0.687 in. long. Primary coolant flowed into the cell, downward around the exhaust tube, into the lower face of the charcoal bed, and upward through the bed and the exhaust tube. The lower portion of the cell was surrounded by a massive aluminum heat sink that served to keep the charcoal cool whenever the cell was temporarily removed from the refrigerant.

Primary coolant was conducted from the primary circuit to the cell and then to the low pressure [IP ( 4 psig)] helium dump tank, as shown in Fig. 4.2-2. The flow originated at the helium outlet duct of the loop-I steam generator, passed through $\sim 12$ ft of 0.20l-in. -ID stainless steel tubing and a pressure reducing valve, and passed on to the radiochemical sampling station via 38 ft of $0.152-i n .-I D$ stainless steel tubing. The pressure reducing valve dropped the pressure from 340 to 35 psig. The coolant entered the gas sampling station through valve HSV 8 and exited through valve HSV16, as shown in Fig. 4.1-1. The coolant was conducted from HSVI6 to the charcoal trap through $15 \mathrm{ft}$ of 1/16-in.-ID copper tubing. The pressure of the coolant was monitored by the pressure gauge shown above valve HV6 in Fig. 4.1-1. The flow rate was measured by a Fischer-Porter rotometer, No. 08F-1/16-20-4, indicated in Fig. 4.2-2.

For a detailed explanation of how coolant is sampled through HSV16, the reader is referred to the manual describing the operation of the Peach Bottom Radiochemical Sampling station. ${ }^{2}$ The manner in which samples were collected on the charcoal traps is described briefly as follows: a charcoal trap was placed in a Dewar containing either dry ice of liquid nitrogen, and the piping to the radiochemical sampling station and the station manifold were purged of stagnant gas by allowing coolant to go directly through the station to the low pressure dump tank while the cell was being cooled. The flow was then diverted through the charcoal trap. After a sample was collected, HSV16 was closed, and the cell was disconnected, removed from the refrigerant, and counted. The cell was then replaced in the refrigerant and recounted at later times to optimize the measurements of the longer-lived fission gases. 
ORNL DWG 76-853R1

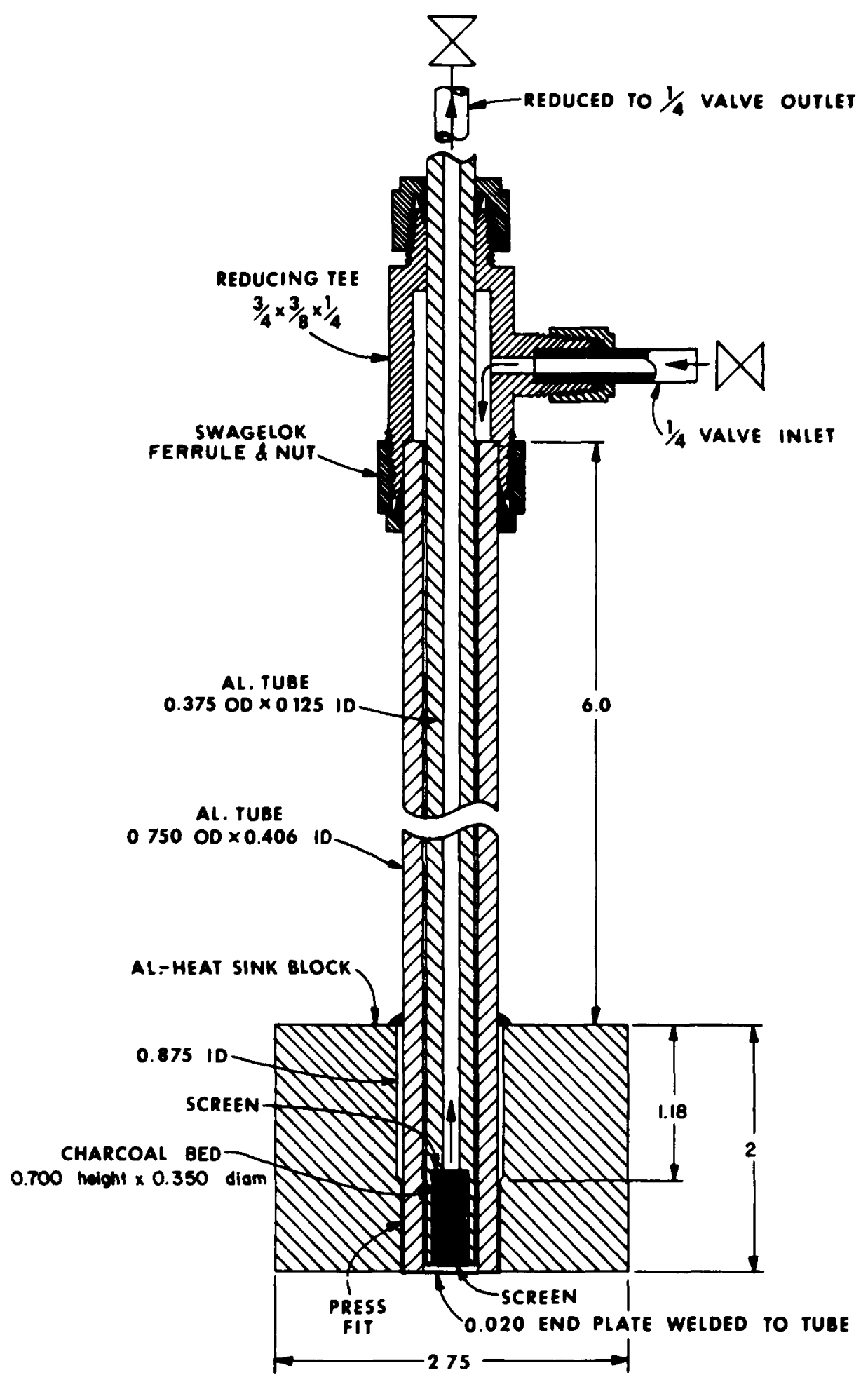

Fig. 4.2-1. Charcoal trap for krypton and xenon in the primary coolant (dimensions in inches). 


$$
\begin{aligned}
\text { A - } & \text { CONNECTION AT HSV - } 16 \\
& \text { (SEE FIG. 4.1) } \\
\text { B - } & \text { CONNECTION TO LP } \\
& \text { HELIUM TANK LINE } \\
& \text { (SEE FIG. 4.1) }
\end{aligned}
$$

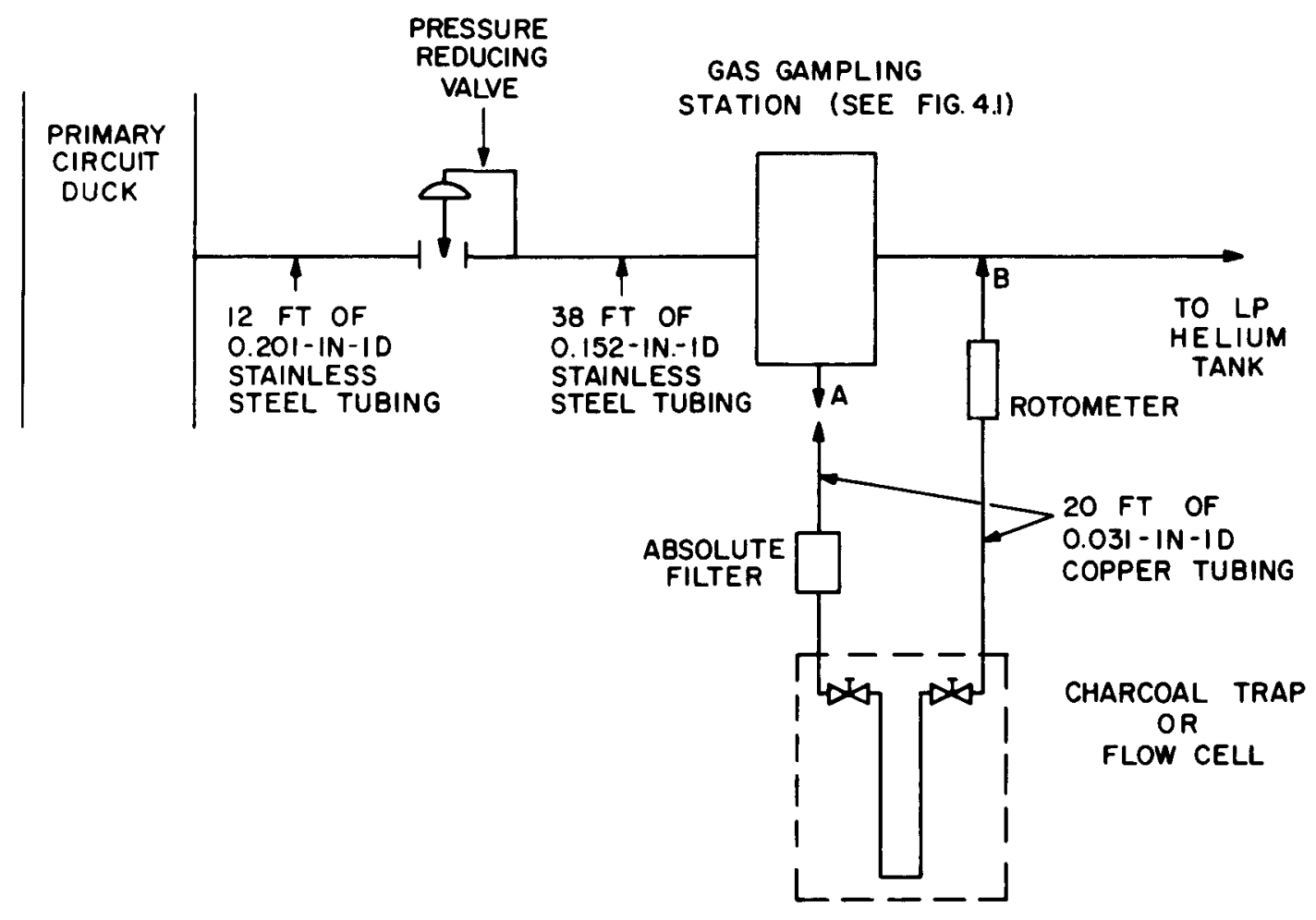

Fig. 4.2-2. Flow diagram of circuit used to sample primary circuit coolant. 
In an effort to decrease possible mutual interferences between $\mathrm{Kr}$ and $X e$ isotopes, samples were collected using either the refrigerants dry ice or liquid nitrogen. Evidence obtained by GAC had suggested that Xe isotopes were strongly adsorbed on charcoal at the temperature of dry ice, whereas $\mathrm{Kr}$ isotopes were not. Thus, it was expected that samples collected with dry ice might improve the measurements of Xe isotopes.

Gamma spectra of the charcoal trap cell were acquired with the cell face located $\sim 0.1 \mathrm{~cm}$ from the $\mathrm{Ge}(\mathrm{Li})$ detector end cap. The face of the charcoal bed was located an additional distance of $\sim 0.1 \mathrm{~cm}$ from the detector. Counting efficiencies were derived from gamma spectra of standard point sources located $0.2 \mathrm{~cm}$ from the detector end cap. This procedure simulated the counting geometry of the face of the charcoal and was used because measurements made by GAC had indicated that fission gases adsorbed mainly on the face of the charcoal rather than throughout the charcoal volume.

Although several samples were collected and measured, difficulties experienced with the gamma spectroscopy equipment prevented all but two from yielding useful results. The concentrations of $\mathrm{Kr}$ and $\mathrm{Xe}$ isotopes derived from these two runs are presented in Table 4.2-3. These samples were collected with the coolant flow rate of $\sim 9.2 \mathrm{scc} / \mathrm{sec}$ and a pressure of $\sim 14$ psig. From the flow rate gas pressures and temperatures and the dimensions of piping, the gas transit time from the primary systems was estimated to be $\sim 210 \mathrm{sec}$. The concentrations of fission gases in Table 4.2-3, expressed as pCi/scc, were corrected for decay during the transit time and, thus, pertain to the coolant in the primary circuit. (Calculational formulae, used to compute the concentrations, are presented in Appendix A.) It should be noted that several of the daughters, notably ${ }^{88} \mathrm{Rb},{ }^{89} \mathrm{Rb}$, and ${ }^{138} \mathrm{Cs}$, were detected in the traps, but no attempt was made to use their activities to derive the parent activities.

\subsubsection{September 1973 measurements}

Samples of core purge gas and primary circuit coolant were analyzed Sept. 11 through 14, 1973. Two grab samples of the core purge gas were obtained with the Peach Bottom Gas Sampling Station. ${ }^{2}$ The samples were 
Table 4.2-3. Concentrations of fission gases measured in primary circuit by adsorption on charcoal (February 19, 1973)

\begin{tabular}{|c|c|c|c|c|c|}
\hline \multirow{2}{*}{$\begin{array}{c}\text { Fission } \\
\text { gas }\end{array}$} & \multicolumn{2}{|c|}{ Measurement $I^{a}$} & \multicolumn{2}{|c|}{ Measurement $2^{b}$} & \multirow{2}{*}{$\frac{\text { Mean }}{\mathrm{pCi} / \mathrm{scc}}$} \\
\hline & $\overline{\mu C i}$ adsorbed & $\mathrm{pCi} / \mathrm{scc}$ & $\overline{\mu C i}$ adsorbed & $\mathrm{pCi} / \mathrm{scc}$ & \\
\hline $\mathrm{Kr}-85$ & $N^{c}$ & & $\mathbb{N D}$ & & \\
\hline$-85 m$ & 0.040 & 3.5 & 0.009 & 2.5 & 3.0 \\
\hline-87 & 0.13 & 12.0 & 0.043 & 12.0 & 12.0 \\
\hline-88 & 0.11 & 10.0 & 0.031 & 8.6 & $9 \cdot 3$ \\
\hline-89 & 0.029 & 22.0 & 0.032 & 33.0 & 27.0 \\
\hline-90 & $\mathrm{ND}$ & & ND & & \\
\hline $\mathrm{Xe}-13 \mathrm{Im}$ & $\mathrm{ND}$ & & ND & & \\
\hline$-133 m$ & $\mathrm{ND}$ & & ND & & \\
\hline-133 & 0.0034 & 0.29 & 0.0021 & 0.57 & 0.43 \\
\hline$-135 m$ & 0.019 & 2.8 & 0.028 & 10.0 & 6.4 \\
\hline-135 & 0.033 & 2.9 & 0.028 & 7.6 & 5.2 \\
\hline-137 & 0.0055 & 2.8 & 0.084 & 64.0 & 33.0 \\
\hline-138 & 0.028 & 4.4 & 0.11 & 41.0 & 23.0 \\
\hline-139 & ND & & ND & & \\
\hline-140 & ND & & $\mathrm{ND}$ & & \\
\hline
\end{tabular}

${ }^{a_{\mathrm{Cell}}}$ cooled with liquid nitrogen. Collection time, $20 \mathrm{~min}$.

$\mathrm{b}_{\mathrm{Cell}}$ cooled with dry ice. Collection time, $380 \mathrm{sec}$.

$c_{\mathrm{ND}}$ indicates that nuclide was not detected. 
collected in 2.7-cc glass vials at a pressure of $-20 \mathrm{~cm} \mathrm{Hg}$ and a temperature of $70^{\circ} \mathrm{F}$. Purge gas was taken from the core purge outlet downstream of the fuel-element purge cooler and conducted through $15 \mathrm{ft}$ of 3/4-in. sched 40 pipe to a valve that reduced the pressure from 340 to 35 psig. The gas was then conducted to the radiochemical sampling station through 71 ft of 0.060-in.-ID stainless steel tubing. After these lines had been purged of stagnant gas, samples were collected with the gas flowing at a rate of 4 liters/min $(67 \mathrm{cc} / \mathrm{sec})$ at a pressure of $25 \mathrm{psig}$ and a temperature of $\sim 70^{\circ} \mathrm{F}$. The time required for the coolant to flow from the purge system to the sampling station was estimated to be $210 \mathrm{sec}$.

Results of these experiments are presented in Table $4.2-4$ both as $\mu \mathrm{Ci}$ measured in the vials and as concentrations, $\mu \mathrm{Ci} / \mathrm{scc}$, derived for the core purge gas. The concentrations, computed as described in Appendix A of this report, were corrected for the $210 \mathrm{sec}$ required for the coolant to flow to the gas sampling station. Unfortunately, this long decay time prevented the detection of the shorter-lived fission gases, ${ }^{90} \mathrm{Kr}$, ${ }^{139} \mathrm{Xe}$, and ${ }^{140} \mathrm{Xe}$, in the purge gas.

Fission gases in the primary circuit coolant were measured by two methods during the september 1973 experiment. The first method made use of the charcoal traps described in Sect. 4.2.2 on the February 1973 experiment. Again, the coolant was taken from the coolant outlet duct of the loop-1 steam generator utilizing the same gas flow circuit as shown in Fig. 4.2-2 and the same Fischer-Porter rotometer. For each of the samples collected in the charcoal traps, the coolant pressure was 12 psig, and the flow rate was estimated to be $9.7 \mathrm{scc} / \mathrm{sec}$. From the dimensions of the piping leading to the trap, the pressures and temperatures of the gas in pipes, and the flow rate, the flow transit time was estimated to be $\sim 200 \mathrm{sec}$.

Three charcoal adsorbed samples were collected employing collection times of $\sim 2 \mathrm{hr}$ and using only liquid nitrogen as the refrigerant. In all other respects, the procedures were the same as those for the February measurements. 
Table 4.2-4. Fission gases observed in Peach Bottom core purge gas via the "grab sample" method

(September 13, 1973)

\begin{tabular}{|c|c|c|c|c|c|}
\hline \multirow[b]{2}{*}{$\begin{array}{l}\text { Fission } \\
\text { gas }\end{array}$} & \multicolumn{2}{|c|}{ Sample I } & \multicolumn{2}{|c|}{ Sample 2} & \multirow[b]{2}{*}{$\begin{array}{c}\text { Mean } \\
\mu \mathrm{Ci} / \mathrm{scc}\end{array}$} \\
\hline & $\begin{array}{l}\mu \mathrm{Ci} \\
\text { in vial }\end{array}$ & $\begin{array}{c}\mu \mathrm{C} i / \overline{s c c} \\
\text { in purge gas } b\end{array}$ & $\begin{array}{l}\mu \mathrm{Ci} \\
\text { in vial }\end{array}$ & $\begin{array}{c}\mu \mathrm{Ci} / \mathrm{scc}^{\mathrm{b}} \\
\text { in purge gas }\end{array}$ & \\
\hline $\mathrm{Kr}-85 \mathrm{~m}$ & 0.095 & 0.052 & 0.092 & 0.050 & 0.051 \\
\hline-85 & $\mathrm{ND}^{\mathrm{C}}$ & & 0.018 & 0.026 & \\
\hline-87 & 0.42 & 0.31 & 0.45 & 0.34 & 0.33 \\
\hline-88 & 0.32 & 0.17 & 0.32 & 0.17 & 0.17 \\
\hline-89 & 0.54 & 0.63 & 0.49 & 0.58 & 0.61 \\
\hline-90 & ND & & ND & & \\
\hline $\mathrm{Xe}-13 \mathrm{Im}$ & $\mathrm{ND}$ & & $\mathrm{ND}$ & & \\
\hline$-133 \mathrm{~m}$ & $\mathrm{ND}$ & & $\mathrm{ND}$ & & \\
\hline-133 & ND & & & & \\
\hline$-135 \mathrm{~m}$ & 0.34 & 0.22 & 0.35 & 0.23 & 0.215 \\
\hline-135 & 0.11 & 0.059 & 0.11 & 0.059 & 0.059 \\
\hline-137 & 0.41 & 0.37 & 0.37 & 0.38 & 0.37 \\
\hline-138 & 0.52 & 0.34 & 0.51 & 0.34 & 0.34 \\
\hline-139 & $\mathrm{ND}$ & & $\mathrm{ND}$ & & \\
\hline-140 & $\mathrm{ND}$ & & $\mathrm{ND}$ & & \\
\hline
\end{tabular}

Activities in vials corrected for decay to time sample was taken.

${ }^{b}$ Concentrations corrected for 210-sec decay time, necessary for purge gas to flow to sampling station. STP denotes temperature of $70^{\circ} \mathrm{C}$ and 1 atm pressure.

$\mathrm{c}_{\mathrm{ND}}$ indicates that radionuclide was not detected.

The amounts of fission gases $(\mu \mathrm{Ci})$ detected in the charcoal traps and their derived concentrations in the primary circuit coolant are presented in Table 4.2-5. Again, it should be noted that the concentrations pertain to the coolant in the steam-generator outlet duct; that is, decay corrections were made for the estimated 200-sec gas transit. As in the case of the February measurements, daughters of fission gases were observed in the traps, but their activities were not used to derive the activities of their parents.

The second method used to determine fission gases in the primary coolant employed a counting cell through which coolant was allowed to flow and be simultaneously counted. This "flow cell, "shown 
Table 4.2-5. Concentrations of fission gases in Loop 1 primary circuit.

coolant collected for approximately two hours on charcoal trap

(September 13, 1973)

\begin{tabular}{|c|c|c|c|c|c|c|c|}
\hline \multirow[b]{2}{*}{ Radionuclide } & \multicolumn{2}{|c|}{ Sample 1} & \multicolumn{2}{|c|}{ Sample 2} & \multicolumn{2}{|c|}{ Sample 3} & \multirow{2}{*}{$\begin{array}{c}\text { Mean } \\
\mathrm{pCi} / \mathrm{cc}^{\mathrm{a}}\end{array}$} \\
\hline & $\mu \mathrm{Ci}$ adsorbed & $\mathrm{pCi} / \mathrm{scc}^{\mathrm{a}}$ & $\mu \mathrm{Ci}$ adsorbed & $\mathrm{pCi} / \mathrm{sec}^{\mathrm{Q}}$ & $\mu \mathrm{Ci}$ adsorbed & $\mathrm{pCi} / \mathrm{scc}^{\mathrm{a}}$ & \\
\hline $\mathrm{Kr}-85 \mathrm{~m}$ & 0.30 & 5.5 & 0.19 & 3.7 & 0.26 & 5.0 & 4.7 \\
\hline-85 & & & ND & & ND & & \\
\hline-87 & 0.70 & 19.0 & 0.42 & 12.0 & 0.59 & 17.0 & 16.0 \\
\hline-88 & 0.75 & 15.0 & 0.44 & 9.5 & 0.62 & 13.0 & 12.0 \\
\hline-89 & 0.036 & 33.0 & 0.030 & 27.0 & 0.012 & 11.0 & 24.0 \\
\hline $\mathrm{Xe}-13 \mathrm{Im}$ & $\mathrm{ND}$ & & $\mathrm{ND}$ & & $\mathrm{ND}$ & & \\
\hline$-133 m$ & $\mathrm{ND}$ & & $\mathrm{ND}$ & & $\mathrm{ND}$ & & \\
\hline-133 & 0.065 & 1.0 & 0.036 & 0.60 & 0.053 & 0.89 & 0.83 \\
\hline$-135 m$ & 0.067 & 6.9 & 0.028 & 2.9 & 0.046 & 4.8 & 4.9 \\
\hline-135 & 0.35 & 5.9 & 0.13 & 2.3 & 0.27 & 4.9 & 4.4 \\
\hline-137 & 0.46 & 27.0 & 0.030 & 17.0 & 0.017 & 9.9 & 18.0 \\
\hline-138 & 0.28 & 32.0 & 0.14 & 16.0 & 0.174 & 20.0 & 23.0 \\
\hline-139 & $\mathrm{ND}$ & & ND & & ND & & \\
\hline-140 & $\mathrm{ND}$ & & ND & & $\mathrm{ND}$ & & \\
\hline
\end{tabular}

${ }^{a}$ Concentrations pertain to coolant in steam generator outlet duct.

$\mathrm{b}_{\mathrm{ND}}$ indicates that radionuclide was not detected. 
schematically in Fig. 4.2-3, had an inner annulus that allowed it to be placed over the end cap of a $\mathrm{Ge}(\mathrm{I} i)$ detector to maximize the detection sensitivity for fission gases. The volume of the cell was $2225 \mathrm{scc}$. The cell was installed in the same way as the charcoal trap.

The motivation for adopting this technique was to achieve an increased detection sensitivity for the shorter-lived fission gases. The shorterlived isotopes, when collected on charcoal, rapidly decay away after the gas flow is stopped and counting has begun. In the flow cell, the shorterlived gases are continuously replaced and can be measured for any arbitrary length of time.

Four separate measurements utilizing the flow cell were made at progressively increasing flow rates; Table 4.2-6 contains a summary of the pertinent flow conditions. Unfortunately, due to an oversight, the coolant pressure was not recorded for these measurements; however, the rotometer reading for the second measurement indicated that the flow rate and, hence, the pressure were very similar to that for the charcoal trap experiments. The results were analyzed on this basis. Since the gas transit times were very small relative to the half-life of ${ }^{88} \mathrm{Kr}(2.8 \mathrm{hr})$, virtually no decay of ${ }^{88} \mathrm{Kr}$ occurred during the transfer. The amount of ${ }^{88} \mathrm{Kr}$ observed in the cell was thus directly proportional to coolant pressure in the cell. Based on the assumption that the pressure in the cell for run 2 was 26.7 psia and that the ${ }^{88} \mathrm{Kr}$ concentration remained constant during the runs, the pressure in the cell for the other three runs may be computed by:

$$
P_{i}=26.7 \operatorname{psia}\left(\frac{A_{i}}{A_{2}}\right) \text {, }
$$

where $P_{i}$ and $A_{i}$ are the pressure, and observed number of microcuries of ${ }^{88} \mathrm{Kr}$ for run $i$ and $\mathrm{A}_{2}$ is the observed number of microcuries in the cell for $\operatorname{man} 2$. The gas transit times for runs 1, 3, and 4 were derived from the ratio of the observed activities of ${ }^{90} \mathrm{Kr}$ and ${ }^{88} \mathrm{Kr}$ in the cell. Since this ratio is independent of pressure, the nuclide ratio in the cell, $R_{c}$, is related to that in the primary circuit, $R_{p}$, by:

$$
R_{c}=R_{p} e\left(\lambda_{88}-\lambda_{90}\right)^{t} \text {, }
$$




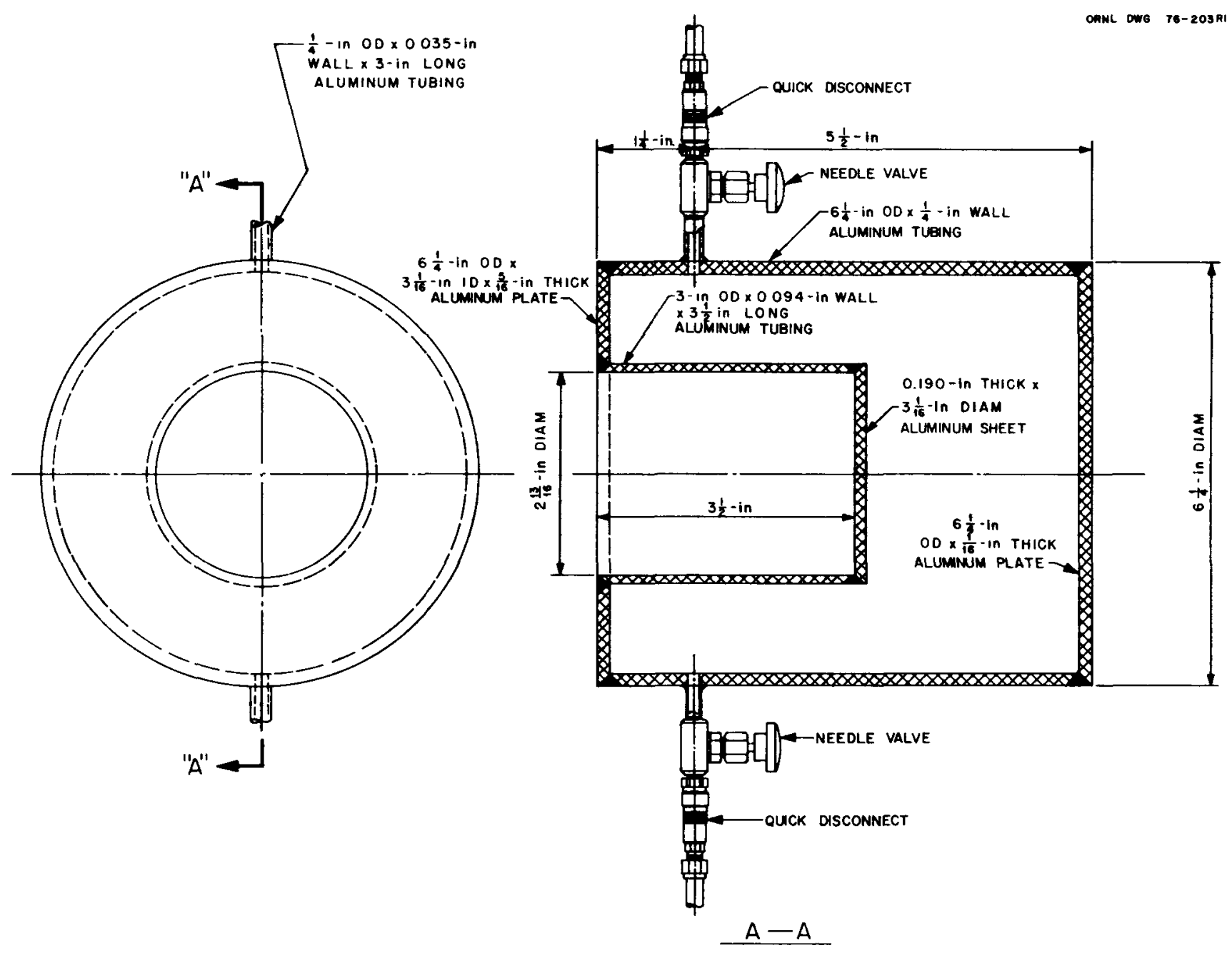

Fig. 4.2-3. "Flow-through" cell for fission gas measurement in the primary coolant. 
Table 4.2-6. Information pertinent to coolant flow for flow cell measurements

\begin{tabular}{|c|c|c|c|c|c|c|c|c|}
\hline \multirow[b]{2}{*}{$\begin{array}{l}\text { Run } \\
\text { No. }\end{array}$} & \multirow[b]{2}{*}{$\begin{array}{l}\text { Rotometer } \\
\text { identification }\end{array}$} & \multirow[b]{2}{*}{$\begin{array}{l}\text { Float } \\
\text { type used }\end{array}$} & \multicolumn{2}{|c|}{ Rotometer range } & \multirow[b]{2}{*}{$\begin{array}{l}\text { Experimental } \\
\text { scale reading }\end{array}$} & \multirow{2}{*}{$\begin{array}{l}\text { Coolant } \\
\text { pressure } \\
\text { (psia) }\end{array}$} & \multirow{2}{*}{$\begin{array}{l}\text { Flow rate } \\
(\mathrm{scc} / \mathrm{sec})\end{array}$} & \multirow{2}{*}{$\begin{array}{l}\text { Gas transit } \\
\text { time } \\
(\mathrm{sec})\end{array}$} \\
\hline & & & $\begin{array}{l}\text { Scale } \\
\text { range }\end{array}$ & $\begin{array}{l}\text { Flow } \\
\text { range }\end{array}$ & & & & \\
\hline 1 & $\begin{array}{l}\text { Fischer Porter } \\
08 \mathrm{~F}-1116-20-4\end{array}$ & Sapphire & $1-20$ & $27-271 \mathrm{scc} / \mathrm{min}$ & 13 & 17.8 & 7.0 & 267 \\
\hline 2 & $\begin{array}{l}\text { Fischer Porter } \\
\text { 08F-1I16-20-4 }\end{array}$ & Stainless steel & $I-20$ & $43-426 \mathrm{scc} / \mathrm{min}$ & 14 & 26.7 & 9.7 & 200 \\
\hline 3 & Brooks 2-65A & Glass & $0.2-1.2$ & $0.2-2 \mathrm{cfh}$ & 1 & 36.0 & 11.3 & 179 \\
\hline 4 & Brooks 2-65A & Glass & & $0.2-2 \mathrm{cfh}^{\mathrm{h}}$ & 2 & 39.0 & 14.9 & 138 \\
\hline
\end{tabular}

${ }^{a}$ See text for method of estimating coolant pressures. 
where $\lambda_{90}$ and $\lambda_{88}$ are the respective decay constants of ${ }^{90} \mathrm{Kr}$ and ${ }^{88} \mathrm{Kr}$, and $t$ is the gas transit time. Neglecting $\lambda_{88}$, because it is very small compared with $\lambda_{90}$, and assuming the relative levels in the primary coolant to remain unchanged, we can write:

$$
\frac{R_{c, i}}{R_{c, 2}}=e^{\lambda_{90}\left(t_{2}-t_{i}\right)},
$$

where the subscripts $i$ and 2 denote runs $i$ and 2, respectively. The gas transit time for the ith mun is thus given by the expression:

$$
t_{i}=t_{2}+\frac{1}{\lambda_{90}} \ln \frac{R_{c, 2}}{R_{c, i}} \text {, }
$$

where the value of $t_{2}$ was assumed to be $200 \mathrm{sec}$, as estimated for the charcoal trap runs.

The amounts of fission gases measured in the flow cells for the four runs and their derived concentrations in the primary circuit are listed in Table 4.2-7. The short-lived fission gases $90_{\mathrm{Kr}}$ (half-life of $33 \mathrm{sec}$ ) and ${ }^{139} \mathrm{Xe}$ (half-life of $40 \mathrm{sec}$ ) were detected in each of the four runs. Xenon-140 (half-life of $14 \mathrm{sec}$ ) was not observed in the first three runs, but its presence in the fourth run was confirmed by the occurrence of six of its photopeaks, all of which yielded similar values for its activity. The gamma spectrum obtained from the fourth run was rather complex and contained $\sim 150$ photopeaks. Fission gas daughters that were observed included ${ }^{88} \mathrm{Rb},{ }^{89} \mathrm{Rb}$, and ${ }^{138} \mathrm{Cs}$. Weak evidence was obtained for the presence of $90 \mathrm{Rb},{ }^{139} \mathrm{Cs}$, and ${ }^{140} \mathrm{Cs}$, that is, one or two photopeaks of the correct energy were observed. Photopeaks corresponding to the energy of the most intense gamma ray of ${ }^{91} \mathrm{Kr}(108.6 \mathrm{keV})$ and ${ }^{91_{\mathrm{Rb}}}(93 \mathrm{keV})$ were observed, however, other gamma rays of these radionuclides were not found. The method used to calibrate the counting efficiency of the cells and the calculational formulae employed to compute the concentrations of fission gases in the coolant are discussed in Appendix A. 
Table 4.2-7. Fission gases measured in primary circuit by flow cell method

(September 13, 1973)

\begin{tabular}{|c|c|c|c|c|c|c|c|c|c|}
\hline \multirow[b]{2}{*}{$\begin{array}{c}\text { Fission } \\
\text { gas }\end{array}$} & \multicolumn{2}{|c|}{ Run 1} & \multicolumn{2}{|c|}{ Run 2} & \multicolumn{2}{|c|}{ Run 3} & \multicolumn{2}{|c|}{ Run 4} & \multirow[b]{2}{*}{$\begin{array}{c}\text { Mean } \\
\mathrm{pCi} / \mathrm{scc}\end{array}$} \\
\hline & $\begin{array}{c}\mathrm{Ci} \text { in } \\
\text { cell }\end{array}$ & $\mathrm{pCi} / \mathrm{scc}$ & $\begin{array}{c}\overline{\mu C i} \text { in } \\
\text { cell }\end{array}$ & $\overline{\mathrm{pCi} / \mathrm{scc}}$ & $\begin{array}{c}\overline{\mu \mathrm{Ci}} \text { in } \\
\text { cell }\end{array}$ & $\mathrm{pCi} / \mathrm{scc}$ & $\begin{array}{l}\mu \mathrm{Ci} \text { in } \\
\text { cell }\end{array}$ & $\mathrm{pCi} / \mathrm{scc}$ & \\
\hline $\begin{array}{c}\mathrm{Kr}-85 \mathrm{~m} \\
-85\end{array}$ & $\begin{array}{c}9.4 E-3 \\
N D^{a}\end{array}$ & 3.6 & $1.1 \mathrm{E}-2$ & 2.9 & $\begin{array}{l}1.7 \mathrm{E}-2 \\
\mathrm{ND}\end{array}$ & $3 \cdot 3$ & $\begin{array}{l}1.8 \mathrm{E}-2 \\
\mathrm{ND}\end{array}$ & 3.2 & 3.2 \\
\hline-87 & $3.4 \mathrm{E}-2$ & 14.0 & $4.4 \mathrm{E}-2$ & 12.0 & $6.7 \mathrm{E}-2$ & 14.0 & $7.1 \mathrm{E}-2$ & 13.0 & 13.0 \\
\hline-88 & $2.8 \mathrm{E}-2$ & 11.0 & $4.0 \mathrm{E}-2$ & 10.0 & $5.4 \mathrm{E}-2$ & 10.0 & $5.6 \mathrm{E}-2$ & 10.0 & 10.0 \\
\hline-89 & $1.2 \mathrm{E}-2$ & 27.0 & $2.7 \mathrm{E}-2$ & 33.0 & $3.8 \mathrm{E}-2$ & 35.0 & $5.3 \mathrm{E}-2$ & 35.0 & 32.0 \\
\hline-90 & $6.9 \mathrm{E}-4$ & 750.0 & $4.2 \mathrm{E}-3$ & 760.0 & $8.9 \mathrm{E}-3$ & 860.0 & $2.7 \mathrm{E}-2$ & 830.0 & 800.0 \\
\hline $\mathrm{Xe}-13 \mathrm{Im}$ & $\mathrm{ND}$ & & $N D$ & & ND & & ND & & \\
\hline$-133 m$ & $\mathrm{ND}$ & & ND & & $4.0 \mathrm{E}-3$ & 0.7 & $6.9 \mathrm{E}-3$ & 1.2 & 0.9 \\
\hline-133 & $\mathrm{ND}$ & & 7.1 E-4 & 0.2 & $1.2 \mathrm{E}-3$ & 0.2 & $1.4 \mathrm{E}-3$ & 0.2 & 0.2 \\
\hline$-135 \mathrm{~m}$ & $1.1 \mathrm{E}-2$ & 6.3 & $1.5 \mathrm{E}-2$ & 5.6 & $2.2 E-2$ & 6.3 & $2.4 \mathrm{E}-2$ & 5.9 & 6.0 \\
\hline-135 & $1.1 \mathrm{E}-2$ & 4.1 & $1.4 \mathrm{E}-2$ & 3.5 & $1.9 \mathrm{E}-2$ & 3.6 & $2.1 \mathrm{E}-2$ & 3.5 & 3.7 \\
\hline-137 & $2.6 \mathrm{E}-2$ & 41.0 & $4.4 \mathrm{E}-2$ & 39.0 & $7.2 \mathrm{E}-2$ & 49.0 & $9.5 \mathrm{E}-2$ & 48.0 & 44.0 \\
\hline-138 & $4.0 \mathrm{E}-2$ & 23.0 & $6.0 \mathrm{E}-2$ & 23.0 & $9.5 \mathrm{E}-2$ & 27.0 & 0.011 & 26.0 & 25.0 \\
\hline-139 & $1.8 \mathrm{E}-3$ & 450.0 & $6.0 \mathrm{E}-3$ & 340.0 & $1.2 \mathrm{E}-2$ & 380.0 & $2.8 \mathrm{E}-2$ & 360.0 & 380.0 \\
\hline-140 & ND & & ND & & $\mathrm{ND}$ & & $1.04 \mathrm{E}-3$ & 2700.0 & 2700.0 \\
\hline
\end{tabular}

$a_{\mathrm{ND}}$ indicates not detected. 


\subsection{Release-to-Birth Rate Ratios of Fission Gases}

If a steady-state level of fission gas in the primary coolant is assumed, the release rate, $R^{\prime}$ (mole/sec) of fission gas to the primary coolant circuit may be estimated from the relation:

$$
\mathrm{R}^{2}=(\lambda+\mathrm{L}) \mathrm{N}_{\mathrm{g}} \mathrm{V}
$$

where

$\lambda=$ decay constant of the fission gas;

$I=$ the fraction of the coolant that enters the core purge system and chemical cleanup plant per second;

$N_{g}=$ fission gas concentration in the primary coolant, mole/scc;

$\mathrm{V}=$ the volume of coolant in the primary circuit, scc.

The concentrations, $\mathbb{N}_{\mathrm{g}}$, were computed from the levels given in $\mathrm{pCi} / \mathrm{scc}$ in Tables 4.2-1, 4.2-3, 4.2-5, and 4.2-7. Since the coolant inventory in the primary circuit was $\sim 900 \mathrm{Ib}$, the coolant entry rate into each of the 804 fuel elements was $\sim 1.1 \mathrm{lb} / \mathrm{hr}$ (purge-gas flow rate), and the coolant entry rate into chemical cleanup plant was $100 \mathrm{Ib} / \mathrm{hr}, \mathrm{V}$ and $\mathrm{I}$ are estimated to be $2.3 \mathrm{E} 9 \mathrm{cc}$ and $3.04 \mathrm{E}-4 / \mathrm{sec}$, respectively.

Birth rates, $B$, were computed from the equation:

$\mathrm{B}=\mathrm{P} \cdot \mathrm{A} \cdot \mathrm{Y}$,

where

$P=$ the reactor power in megawatts,

$A=$ moles of fissions per second per megawatt, $3.12 \mathrm{El6} / 6.023 \mathrm{E} 23$,

$Y=$ the fission yield of the fission gases (atoms formed per fission).

During each measurement, the power of the reactor was $107 \mathrm{MW}$. Fission yields chosen for the calculations were those tabulated and recommended by Meek and Rider for the fission of ${ }^{235} \mathrm{U}$ by thermal neutrons. 4 No attempt was made to take into account the fissioning of $233_{U}$ or the fission produced by epithermal neutrons.

Values of $\mathrm{R}^{\prime} / \mathrm{B}$ for the primary circuit, calculated using Eqs. (5) and (6) for each of the experiments in which fission gases were measured (Dec. 15, 1970, Feb. 19, 1973, and Sept. 13, 1973), are given in 
Table 4.2-8. Also presented are the inventories of fission gases in millicuries in the total coolant.

The release rate, $R$, of a fission gas into the fuel-element purge system was computed from the equation:

$$
R=N_{g}^{F},
$$

where

$\mathrm{Ng}=$ fission gas concentration in purge gas, mole/scc,

$\mathrm{F}=$ the purge-gas flow rate, $7.18 \mathrm{E} 5 \mathrm{scc} / \mathrm{sec}$.

To compute the release rate from the fuel into the purge gas by the expression above, it was assumed the amounts of fission gases that entered the fuel elements from the primary circuit and the release rates through the fuel element sleeves to the primary circuit were negligible and that no fission gases returned to the reactor from the purge system cleanup plant.

Release rate-to-birth rate ratios, $R / B$, for the purge gas calculated from fission gas concentration measured sept. 13, 1973, are presented in Table 4.2-9.

\subsection{Discussion of Fission Gas Determination}

The experience gained and results found in this study clearly show that fission gases in an HTGR can best be measured by counting a flowing stream of the coolant and/or purge gas. If the transit time from the primary system is sufficiently short, all of the fission gases present in the source gas will be available at the detector. The detection of the shorter-lived isotopes may be enhanced by acquiring gamma spectra for any arbitrarily long time. The detection of the very short-lived fission gases $90 \mathrm{Kr},{ }^{139} \mathrm{Xe}$, and ${ }^{140} \mathrm{Xe}$ was demonstrated by this method using the flow cells described in sect. 4.2. Except as noted below, the presence of the shorterlived gases do not interfere with the measurement of the longer-lived isotopes. Although a few fission-gas daughters were observed in the flow cells, they neither build up in the cells nor interfere with the measurements of their parents. 
Table 4.2-8. Release rate-birth rate ratios, $\mathrm{R}^{\prime} / \mathrm{B}$, and activity inventories of fission gases in the primary circuit (release to coolant)

\begin{tabular}{|c|c|c|c|c|c|c|c|c|}
\hline \multirow[b]{2}{*}{$\begin{array}{c}\text { Fission } \\
\text { gas }\end{array}$} & \multicolumn{2}{|c|}{ December 5, 1970} & \multicolumn{2}{|c|}{ February 19, 1973} & \multicolumn{2}{|c|}{ September 13, 1973} & \multicolumn{2}{|c|}{ September 13, 1973} \\
\hline & $\mathrm{R}^{\prime} / \mathrm{B}$ & $\begin{array}{l}\text { Activity } \\
\text { inventory } \\
\text { (mCi) }\end{array}$ & $R^{\prime} / B^{a}$ & $\begin{array}{l}\text { Activity } \\
\text { inventory } \\
\quad(\mathrm{mCi})\end{array}$ & $R^{\prime} / B^{b}$ & $\begin{array}{l}\text { Activity } \mathrm{b} \\
\text { inventory } \\
(\mathrm{mCi})\end{array}$ & $R^{\prime} / B^{c}$ & $\begin{array}{l}\text { Activity } \\
\text { inventory } \\
(\mathrm{mci})\end{array}$ \\
\hline $\mathrm{Kr}-85$ & $\mathrm{ND}^{\mathrm{d}}$ & & & & $\mathrm{ND}$ & & $\mathbb{N D}$ & \\
\hline$-88 m$ & $\mathrm{ND}$ & & 4. $9 \mathrm{E}-8$ & 7.8 & $7.7 \mathrm{E}-8$ & 12.0 & $5.2 \mathrm{E}-8$ & 8.4 \\
\hline-87 & $1.8 \mathrm{E}-9$ & 1.6 & $3.8 \mathrm{E}-8$ & 31.0 & $5.1 E-8$ & 42.0 & 4.2E-8 & 34.0 \\
\hline-88 & IS $\mathrm{e}^{-}$ & & $3 \cdot 7 \mathrm{E}-8$ & 24.0 & $4.8 \mathrm{E}-8$ & 31.0 & 4. OE-8 & 26.0 \\
\hline-89 & $2.83 E-9$ & 12.0 & $1.8 \mathrm{E}-8$ & 71.0 & $1.6 \mathrm{E}-8$ & 63.0 & $2.1 \mathrm{E}-8$ & 84.0 \\
\hline-90 & $8.1 E-9$ & 36.0 & $\mathrm{ND}$ & & ND & & $5 . \mathrm{OE}-7$ & 2100.0 \\
\hline $\mathrm{Xe}-13 \mathrm{Im}$ & $\mathrm{ND}$ & & ND & & ND & & ND & \\
\hline$-133 m$ & $\mathrm{ND}$ & & ND & & $\mathrm{ND}$ & & 1. OE-6 & 2.4 \\
\hline-133 & $\mathrm{ND}$ & & $3 \cdot 3 E-8$ & 1.1 & $6.5 \mathrm{E}-8$ & 2.2 & $1.5 \mathrm{E}-8$ & 0.5 \\
\hline$-135 \mathrm{~m}$ & ND & & $2.4 E-8$ & 17.0 & $1.8 \mathrm{E}-8$ & 13.0 & $2 \cdot 2 \mathrm{E}-8$ & 16.0 \\
\hline-135 & $\mathrm{ND}$ & & $3.2 E-8$ & 14.0 & $2.7 \mathrm{E}-8$ & 12.0 & $2 \cdot 3 \mathrm{E}-8$ & 9.7 \\
\hline-137 & ND & & $1.7 \mathrm{E}-8$ & 86.0 & $9.4 \mathrm{E}-9$ & 47.0 & $2 \cdot 3 \mathrm{E}-8$ & 110.0 \\
\hline-138 & $5.9 \mathrm{E}-9$ & 27.0 & $1.4 \mathrm{E}-8$ & 60.0 & $1.4 \mathrm{E}-8$ & 60.0 & $1.6 \mathrm{E}-8$ & 60.0 \\
\hline-139 & $\mathrm{ND}$ & & ND & & ND & & 2.2E-7 & 1000.0 \\
\hline-140 & ND & & ND & & ND & & $2.1 E-6$ & 7100.0 \\
\hline
\end{tabular}

${ }_{b}^{a}$ Computed from mean activity values tabulated in Table 4.2-3.

Computed from mean activity values tabulated in Table 4.2-5. Computed from mean activity values tabulated in Table 4.2-7. $a_{N D}$ indicates not detected.

Internal standard. 
Table 4.2-9. Release rate-to-birth rate ratios of fission gases in purge system, $R / B$

\begin{tabular}{lc}
\hline $\begin{array}{c}\text { Fission } \\
\text { gas }\end{array}$ & $\begin{array}{c}\text { September } \\
\mathrm{R} / \mathrm{B}^{\mathrm{a}}\end{array}$ \\
\hline $\mathrm{Kr}-85$ & $\mathrm{ND}^{\mathrm{b}}$ \\
-85 & $7 \cdot 2 \mathrm{E}-4$ \\
-87 & $6.8 \mathrm{E}-4$ \\
-88 & $5 \cdot 5 \mathrm{E}-4$ \\
-89 & $2 \cdot 9 \mathrm{E}-5$ \\
-90 & $\mathrm{ND}$ \\
$\mathrm{Xe}-13 \mathrm{~m}$ & $\mathrm{ND}$ \\
$-133 \mathrm{~m}$ & $\mathrm{ND}$ \\
-133 & $\mathrm{ND}$ \\
$-135 \mathrm{~m}$ & $2.2 \mathrm{E}-4$ \\
-135 & $3.4 \mathrm{E}-4$ \\
-137 & $1.7 \mathrm{E}-5$ \\
-138 & $5.4 \mathrm{E}-5$ \\
-139 & $\mathrm{ND}$ \\
-140 & $\mathrm{ND}$ \\
\hline${ }^{2}$ Computed from mean activity concentrations \\
given in Table 4.2-4. \\
$\mathrm{b}_{\text {ND indicates not detected. }}$
\end{tabular}

The collection and counting of fission gases on a charcoal trap is unsuitable for measuring the shorter-lived isotopes because of their decay to nonmeasurable levels both during collection and counting. The trap could be counted during the collection, but the gamma spectrum would rapidly become very complex due to the buildup of all the fission gas daughters. This method would also complicate the derivation of fission gas concentrations. In spite of these drawbacks, the charcoal trapping method has the decided advantage over other methods in that very large amounts of coolant can be sampled with a consequentially high detection sensitivity for the longer-lived fission gases. The shorter-lived fission gases and their daughters can be allowed to decay before the longer-lived isotopes are measured, thus optimizing the determination of the longerlived gases. This procedure could have been, but was not, used in this study for the measurement of ${ }^{85} \mathrm{Kr}\left(t_{1 / 2}=10.7\right.$ years $)$ and $13 \mathrm{~lm}_{\mathrm{Xe}}\left(t_{1 / 2}=\right.$ 
12 days); neither of these gases were determined in this study. Both of these radionuclides are formed in fission with low yields, and the branching ratios of their gamma rays are also low. Unless a sample is allowed to decay $~ 20$ to $30 \mathrm{hr}$ before measurement, the $166-\mathrm{keV}$ gamma ray of ${ }^{88} \mathrm{Kr}$ interferes with the detection of ${ }^{13} \mathrm{Im}_{\mathrm{Xe}}$. Provided that a sufficiently large sample is obtained, both ${ }^{85} \mathrm{Kr}$ and ${ }^{13} 1_{\mathrm{Xe}}$ could be measured after a decay of 1 to 2 weeks.

The accuracy with which fission gases were measured in this study is difficult to evaluate. Although, in a few instances, certain activities determined in separate runs during the same experiment differed significantly, for example, ${ }^{138} \mathrm{Xe}$ measured in Feburary 1973 (Table 4.2-3), results were generally in good agreement. Counting-statistics errors normally were a few percent or less. In the case of the charcoal trap measurements, the amounts of fission gases collected, and thus, the concentrations derived for the long-lived fission gases were directly dependent on gas pressure and flow rate. Concentrations of long-lived gases measured with the flow cells were based on the assumption that the coolant pressure in the second run was equal to the pressure measured for the charcoal trap collections (see September 1973 measurements). We believe that the pressures and flow rate used to derive concentrations in all of the experiments were probably uncertain by no more than a factor of 2 and that the derived concentrations of long-lived fission gases are probably comparably accurate.

The concentrations derived for the shorter-lived fission gases depend exponentially on the gas flow transit times. The reader will note that the activity concentrations derived for ${ }^{90} \mathrm{Kr},{ }^{139} \mathrm{Xe}$, and ${ }^{140} \mathrm{Xe}$ in the flow cell experiments (Table 4.2-7) and the release-to-birth rate ratios of these radionuclides (Table 4.2-8) are considerably larger than for the other fission gases. The explanation of these results is clearly that the transit times were overestimated, resulting in excessive decay corrections for the short-lived gas activities. It is, in fact, to be expected that no fission gases as short lived as $90_{\mathrm{Kr}}$ would have sufficient time to migrate through the fuel element sleeves and into the primary circuit. The source of the shorter-lived fission gases, as well as a part of the Ionger-lived gases, in the primary circuit is thus probably "tramp fuel" 
outside the fuel element sleeves. If the gas pressures and transit times

were accurately known, the activities observed for ${ }^{90} \mathrm{Kr},{ }^{139} \mathrm{Xe}$, and ${ }^{140} \mathrm{Xe}$ might be used to compute the levels of tramp fuel in the reactor.

\section{References for Section 4}

1. R. F. Turner, R. D. Burnette, and W. J. Scheffel, HTGR Fuel Performance in the Peach Bottom Reactor, Gulf-GA-Al2675 (July 9, 1973).

2. Operations Manual for the Radiochemical Sampling System for the Peach Bottom HTGR, GA-5774 (Oct. 30, 1964).

3. Letter from M. J. Cooney, Philadelphia Electric Co., to H. J. de Nordwall, Jan. 21, 1971.

4. M. E. Meek and B. F. Rider, Compilation of Fission Product Yields, Va.1lecitos Nuclear Center, NEDO-12154-1, 74NED6 Class 1 (Jan. 26, 1974).

5. ANALYSES OF DUST COLIECTED IN THE CYCLONE SEPARATORS AND FROM THE EXTERIOR SURFACE OF THE FUEI EIEMEITS

\subsection{Dust Collection Method}

Each of the two primary coolant circulators was provided with a 12-in.diam bypass line, the primary purpose of which was to allow compressor operation at $20 \%$ speed with the suction and discharge valves closed. A second use for this bypass line was to provide a convenient location for passing a portion of the primary coolant flow through a cyclone separator for the continuous removal of dust. This was accomplished by providing the 12-in. bypass line with a 4-in. bypass leading to a cyclone separator, shown in Fig. 5.1-1 taken from ref. 1. When the valve in the 12-in. bypass line was closed, as it normally was during reactor operation, $2475 \mathrm{lb} / \mathrm{hr}$ of helium flowed through each dust separator, impelled by the pressure drop across the main compressor. This amounted to about $1 \%$ of the primary flow in each of the two main loops.

The separators, manufactured by the Centrifix Corporation, Houston, Texas, consisted of a shell with a 12-3/4-in. OD in which a Helicord 
ORNL DWG. $76-1248$

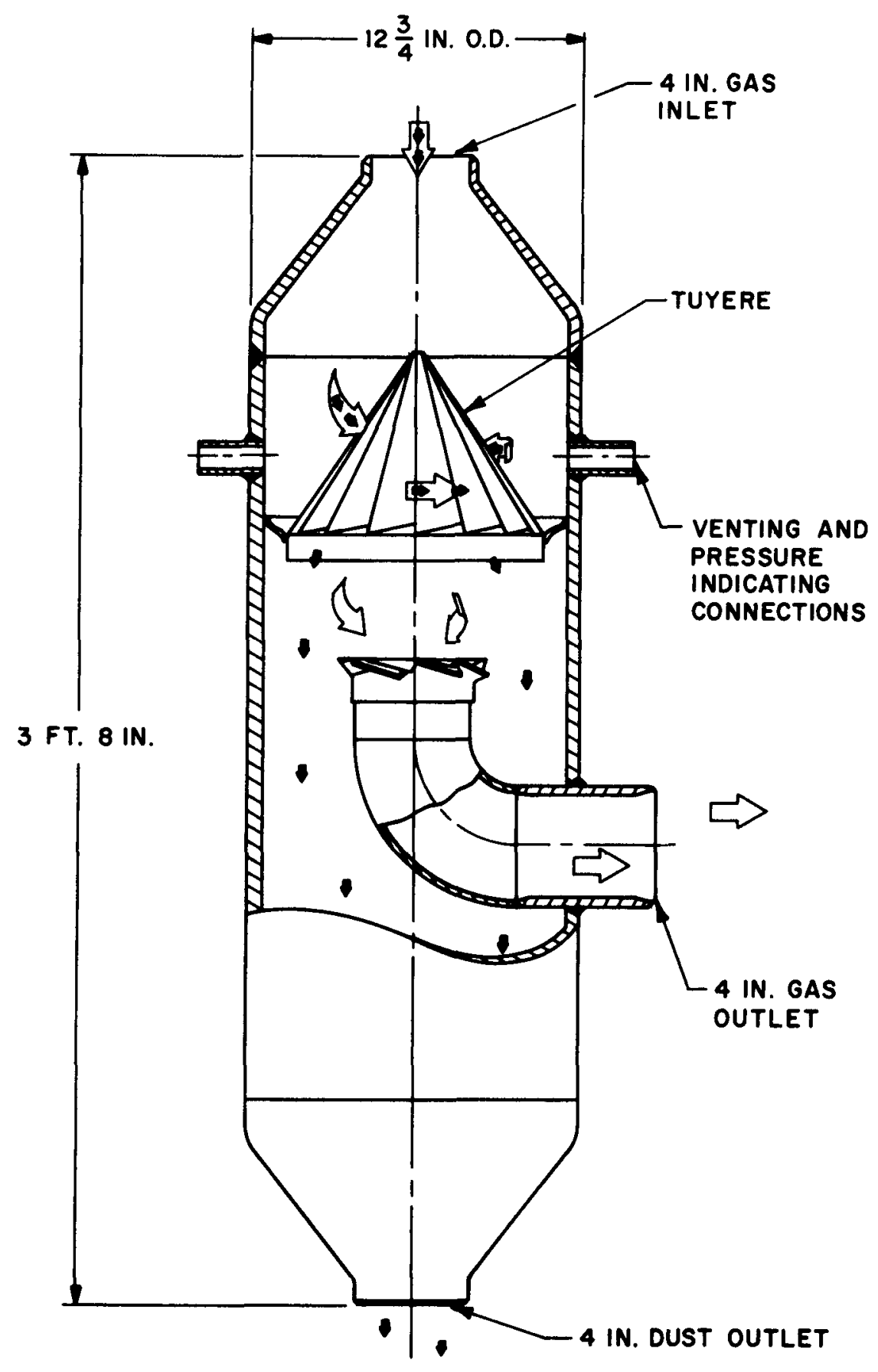

Fig. 5.1-1. Main 10op dust separator. 
Tuyère was mounted to impart a radial velocity to the incoming gas flow. Particles entrained in the gas flow tended to gather at the periphery and fall to the bottom of the filter. The outlet pipe was positioned below the center of the tuyère where the concentration of dust is reduced. The gas inlet and outlet pipes consisted of 4 -in. sched 80 pipe. Two I-in. connections were located in the space above the tuyère for pressure measurements and venting to the IP tank.

The dust collector was located approximately $10 \mathrm{ft}$ below the cyclone separator and was joined to the filter by a 2-in. pipe which passed through the concrete floor to the room below. The collector consisted of a 12-in.$O D$ pipe that was $8 \mathrm{in.} \mathrm{Iong} \mathrm{with} \mathrm{reducers} \mathrm{in} \mathrm{the} \mathrm{ranges} \mathrm{of} 12$ to $4 \mathrm{in}$. and 4 to 2 in. at either end to mate with the inlet and outlet block valves.

The cyclone separators were designed for $92 \%$ removal efficiency for $5-\mu \mathrm{m}$ size particles and $60 \%$ removal efficiency for $0.5-\mu \mathrm{m}$ size particles. ${ }^{2}$ These two points are shown as circles in Fig. 5.1-2, where an approximate relation between separator efficiency and particle size is shown. Curve A in the figure is drawn through the design points with an approximate dependency on particle size, indicated by ref. 3, typical for a commercial cyclone separator. Curve B is drawn with the same general shape, but instead through the square data points representative of preoperational tests obtained with the separators.

On three occasions during shutdown intervals in Core 2 operation, the contents of the dust collectors beneath the cyclone separators were acquired for analysis. While these provide an important view regarding the dust environment within the primary coolant system, several impediments must be noted in attempting to quantitatively interpret these data.

1. The pipe connectors between the 4-in. bypass line containing the dust collectors and the 28-in.-diam primary duct were not designed for the acquisition of representative samples from the primary coolant. The bypass flow should have been drawn off isokinetically from the primary flow. Instead, both the 12-in. tap into the main duct and the 4-in. tap into larger bypass line employed conventional 
ORNL DWG. 76-1250

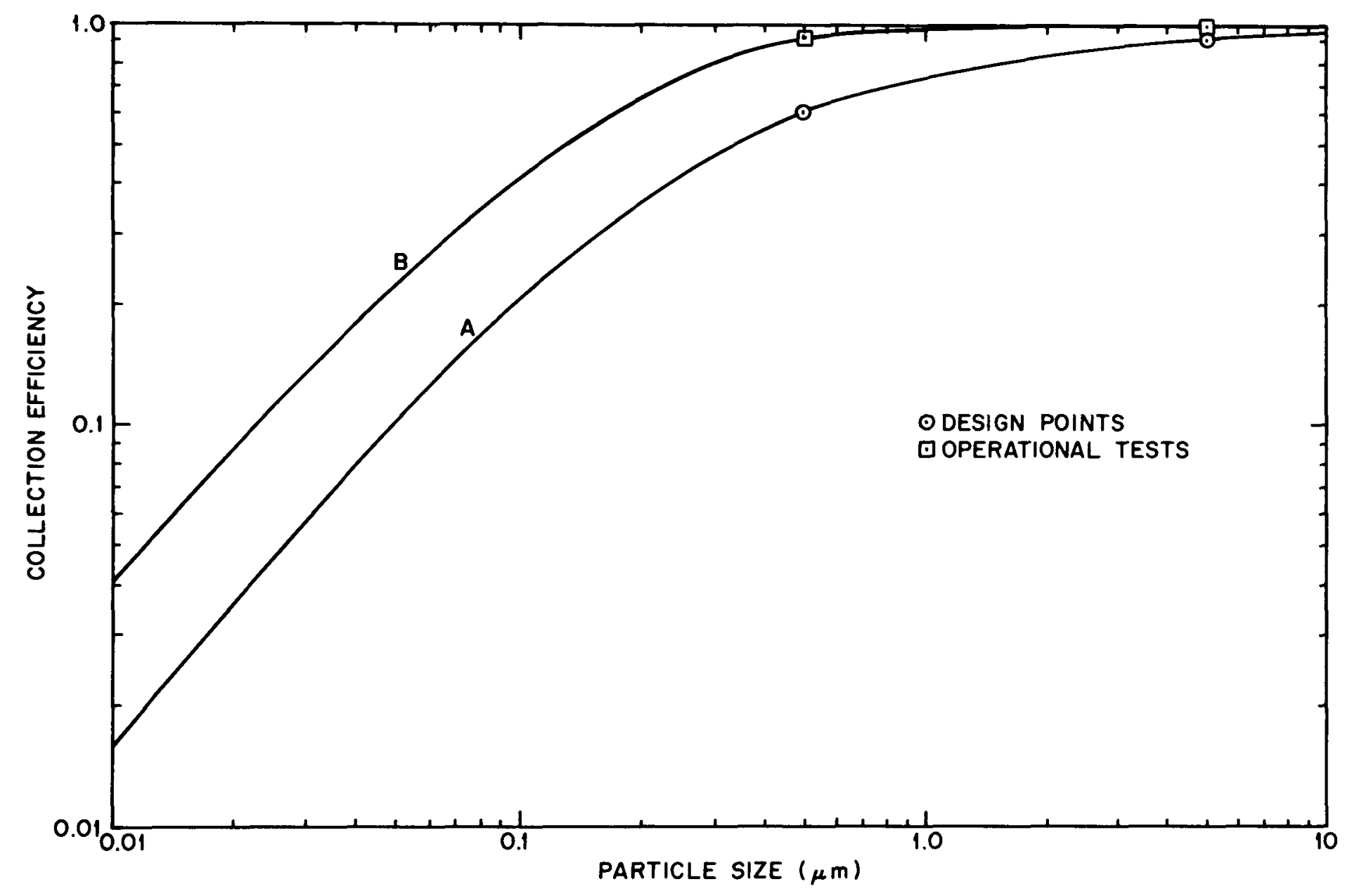

Fig. 5.1-2. Cyclone separator collection efficiency. 
connectors which undoubtedly yielded nonrepresentative dust samples.

2. We have no indication of the state of agglomeration of the circulating particles. As collected, they were highly agglomerated, but they could have been nonagglomerated in the primary system, and thus, the effective circulating particle size may have been much smaller than the measured value. The significance here is that the separator efficieny falls rapidly for sizes less than $\sim 5 \mu \mathrm{m}$. Hence, collector efficiency values must be taken into account in the calculation of loop dust concentration levels if a significant portion of the circulating dust was smaller than $\sim 5 \mu \mathrm{m}$.

\subsection{Summary of Cyclone Separator Dust Data}

Three samples, labeled D2, D3, and D4, were acquired during Core 2 operation at the times indicated in Table 5.2-1. The sampling period for D2 was initiated at the start of Core 2 operation and extended to the first shutdown period. The total sample mass collected was $160 \mathrm{~g}$ from the combined contents of loop 1 and 2 dust collectors. The listed mass of $80 \mathrm{~g}$ for loop 1 dust collector is assumed to be one half of the combined sample. Samples D3 and D4 were collected solely from the loop 1 dust collector.

Tables 5.2-2 through 5.2-4 list the observed particle size distributions for dust samples D2, D3, and D4 obtained by sieve analysis and the observed radionuclide levels in the composite sample and each size fraction.

Tables 5.2-5 and 5.2-6 list the major nonradioactive elements found in dust samples D3 and D4. Analyses for elements Al through Zr were obtained by the spark emission spectrographic method; the total carbon was obtained by burning the sample in oxygen and collecting $\mathrm{CO}_{2}$; total oxygen was obtained by heating at $2200^{\circ} \mathrm{C}$ in excess carbon and collecting the off-gas in a Leco analyzer. The conditions for the oxygen determination were sufficiently reactive to ensure reduction of both silica and alumina in the sample. 
Table 5.2-1. Dust sampling times and sample sizes

\begin{tabular}{|c|c|c|c|c|}
\hline Sample & Collection dates & $\begin{array}{l}\text { Or } \\
\text { (Real time, } \\
\text { (day) }\end{array}$ & $\begin{array}{l}\text { perating time } \\
\text { less shutdown intervals ) } \\
\qquad(\mathrm{sec})\end{array}$ & $\begin{array}{l}\text { Sample mass } \\
\text { from loop } 1 \\
\text { (g) }\end{array}$ \\
\hline D2 & July 15, 1970 ${ }^{2}-A p r . ~ 24,1971$ & 276 & $23 \cdot 9 \mathrm{E} 6$ & $80.0^{b}$ \\
\hline D3 & June 18, 1971-Sept. 14, 1973 & 536 & $46.3 \mathrm{E} 6$ & 110.0 \\
\hline D4 & $\operatorname{Jan}$. 5, 1973-Oct. 31, $1974^{\mathrm{C}}$ & 256 & $22.1 E 6$ & 15.3 \\
\hline
\end{tabular}

${ }^{a}$ Core 2 startup date.

${ }^{b}$ One-half of the combined loop 1 and loop 2 samples.

${ }^{c}$ Core 2 shutdown. 
Table 5.2-2. Dust sample D2: Particle size distribution by sieve analysis and gamma emitting radionuclides concentrations ( $\mu \mathrm{Ci} / \mathrm{g}$, April 24,1971 )

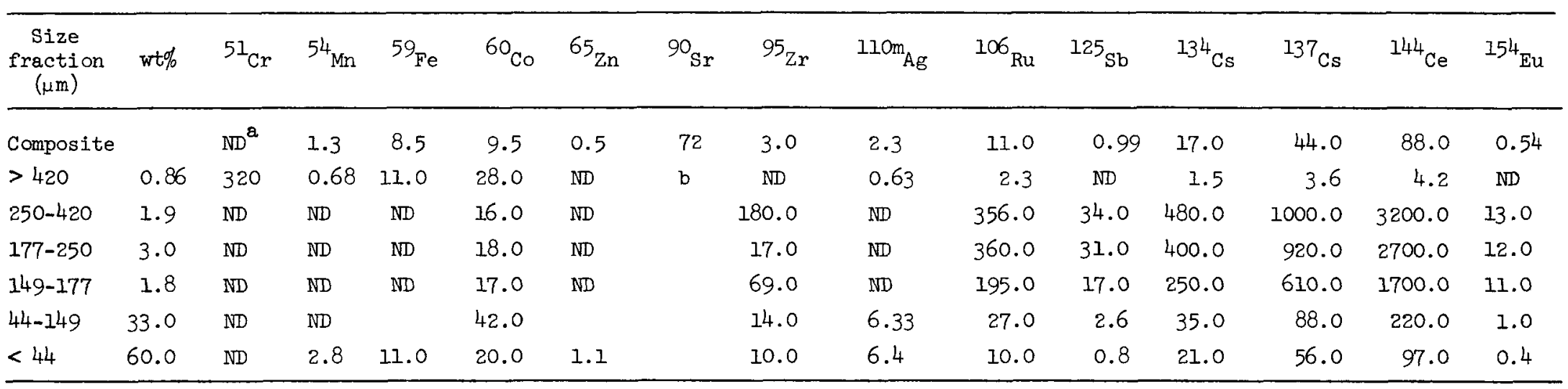

$a_{\mathrm{ND}}$ indicates not detected.

$b_{\text {Blank indicates not measured. }}$ 
Table 5.2-3. Dust sample D3: Particle size distribution and radionuclide concentrations.

( $\mu \mathrm{Ci} / \mathrm{g}$, September 14, 1973)

\begin{tabular}{|c|c|c|c|c|c|c|c|c|c|c|c|c|c|c|c|}
\hline $\begin{array}{l}\text { Size } \\
\text { fraction } \\
\quad(\mu \mathrm{m})\end{array}$ & $\mathrm{wt} \%$ & ${ }^{54} \mathrm{Mn}$ & ${ }^{59} \mathrm{Fe}$ & ${ }^{60} \mathrm{Co}$ & ${ }^{95} \mathrm{Zr}$ & $90 \mathrm{Sr}$ & ${ }^{106} \mathrm{Ru}$ & $110 \mathrm{~m}_{\mathrm{Ag}}$ & $123 \mathrm{~m}_{\mathrm{Te}}$ & $125 \mathrm{~m}_{\mathrm{Te}}$ & $125_{\mathrm{Sb}}$ & ${ }^{134} \mathrm{Cs}$ & ${ }^{137} \mathrm{Cs}$ & ${ }^{144} \mathrm{Ce}$ & ${ }^{154} \mathrm{Eu}$ \\
\hline Composite & & 1.4 & 311 & 61 & 2.7 & 11.0 & $b$ & 6.4 & 0.002 & 0.8 & 0.5 & 6.6 & 30.0 & 18.0 & \\
\hline$>420$ & 2.9 & 1.9 & & 190 & & 0.18 & & $\leq 3.0$ & & & & 0.38 & 1.6 & $\leq 3.0$ & \\
\hline $250-420$ & 3.1 & 8.2 & & 1470 & & 62.0 & & 35.0 & & & & 9.6 & 48.0 & 25.3 & \\
\hline $177-250$ & 3.6 & 2.7 & & 64 & & 6.6 & 18 & $\leq 2.0$ & & & & 30.0 & 145.0 & 140.0 & \\
\hline $149-177$ & 2.2 & $\leq 2.0$ & & 620 & & 51.0 & & $\leq 5.0$ & & & & 22.0 & 123.0 & 99.0 & 1.0 \\
\hline $44-149$ & 36.8 & 0.63 & & 67 & & 17.0 & & 2.5 & & & & 5.9 & 32.0 & 19.0 & 0.2 \\
\hline$<44$ & 51.6 & 1.2 & & 75 & & 11.0 & & 3.1 & & & & 7.0 & 32.0 & 9.1 & \\
\hline
\end{tabular}

${ }^{a}$ Size fractions were obtained by the use of wire-screen sieves.

$\mathrm{b}_{\text {Blank indicates not measured. }}$ 
Table 5.2-4. Dust sample D4: Particle size distribution and radionuclide concentrations

$$
(\mu \mathrm{Ci} / \mathrm{g} \text {, October 31, 1974) }
$$

\begin{tabular}{|c|c|c|c|c|c|c|c|c|}
\hline $\begin{array}{l}\text { Size } \\
\text { fraction } \\
(\mu \mathrm{m}) \\
\end{array}$ & $\mathrm{wt} \%$ & ${ }^{54} \mathrm{Mn}$ & ${ }^{60} \mathrm{Co}$ & ${ }^{90} \mathrm{Sr}$ & $110 \mathrm{~m}_{\mathrm{Ag}}$ & ${ }^{134} \mathrm{Cs}$ & $137_{\mathrm{Cs}}$ & ${ }^{144} \mathrm{Ce}$ \\
\hline Composite & $\mathrm{b}$ & 4.0 & 460 & 29 & 4.2 & 99 & 120 & 1.6 \\
\hline$>450$ & 7.2 & 5.4 & 310 & 0.16 & $\leq 2.0$ & 12 & 9 & $\leq 2$ \\
\hline $250-420$ & 3.0 & 5.6 & 275 & 94 & $\leq 2.0$ & 40 & 90 & 200 \\
\hline $177-250$ & 3.6 & 7.8 & 400 & 79 & $\leq 3.0$ & 45 & 174 & 50 \\
\hline $149-177$ & 2.6 & 4.3 & 1000 & 44 & $\leq 5.0$ & 60 & 175 & 86 \\
\hline $44-149$ & 60.0 & 2.3 & 390 & 26 & 3.42 & 101 & 112 & 10 \\
\hline$<44$ & 23.6 & $3 \cdot 3$ & 140 & 28 & 4.89 & 151 & 156 & 6 \\
\hline
\end{tabular}

${ }^{a}$ Size fractions were obtained by the use of wire screen sieves.

$\mathrm{b}_{\text {Blank indicates not measured. }}$ 
Table 5.2-5. Dust sample D3: elemental composition, ${ }^{a, b}$ wt\%

\begin{tabular}{|c|c|c|c|c|c|c|c|c|c|c|}
\hline $\begin{array}{c}\text { Size } \\
\text { fraction }\end{array}$ & $\mathrm{Al}$ & $\mathrm{Cr}$ & $\mathrm{Cu}$ & $\mathrm{Fe}$ & $\mathrm{Mn}$ & Mo & $\mathrm{Ni}$ & $\mathrm{Ti}$ & $\mathrm{C}$ & 0 \\
\hline Composite & 0.05 & 0.10 & 0.08 & 18.0 & 0.21 & 0.07 & 0.31 & 0.02 & 49.9 & 5.00 \\
\hline$>420$ & 0.05 & 5.29 & 0.35 & 30.6 & 0.54 & $<0.09$ & 7.15 & 0.02 & 10.8 & 2.44 \\
\hline $250-420$ & 0.08 & 0.53 & 0.05 & 16.7 & 0.38 & $<0.1$ & 1.44 & 0.07 & 60.0 & 4.61 \\
\hline $177-250$ & 0.08 & 0.21 & 0.09 & 9.63 & 0.29 & $<0.07$ & 0.35 & 0.07 & 58.4 & \\
\hline $149-177$ & 0.13 & 0.16 & 0.03 & 11.27 & 0.23 & $<0.12$ & 0.33 & 0.03 & 36.9 & 3.99 \\
\hline $44-149$ & 0.07 & 0.16 & 0.07 & 21.8 & 0.32 & 0.08 & 0.34 & 0.03 & 41.7 & 4.92 \\
\hline$<44$ & 0.06 & 0.09 & 0.12 & 18.9 & 0.09 & 0.07 & 0.30 & 0.02 & 51.9 & 3.94 \\
\hline
\end{tabular}

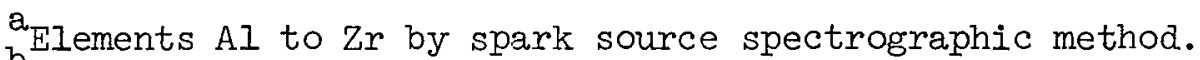

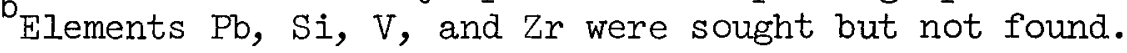


Table 5.2-6. Dust sample D: elemental composition, ${ }^{\mathrm{a}}$ wt\%

\begin{tabular}{lllllllllll}
\hline $\begin{array}{c}\text { Size } \\
\text { fraction }\end{array}$ & $\mathrm{Al}$ & $\mathrm{Cr}$ & $\mathrm{Cu}$ & $\mathrm{Fe}$ & $\mathrm{Mn}$ & $\mathrm{Mo}$ & $\mathrm{Ni}$ & $\mathrm{Ti}$ & $\mathrm{C}$ & 0 \\
\hline Composite & $\mathrm{ND}^{\mathrm{b}}$ & 0.35 & 0.17 & 25.5 & 0.27 & 0.22 & 2.65 & 0.08 & 38.8 & 3.5 \\
$>420$ & $\mathrm{ND}$ & 8.5 & 0.09 & 35.9 & 0.41 & 0.24 & 9.2 & & 5.8 & 9.6 \\
$250-420$ & 0.11 & 1.83 & 0.07 & 13.1 & 0.28 & 0.10 & 2.80 & 0.02 & 17.8 & 3.6 \\
$177-250$ & 0.38 & 1.14 & 0.08 & 21.8 & 0.23 & & 3.22 & 0.06 & 33.1 & 3.3 \\
$149-177$ & 0.40 & 1.01 & 0.14 & 22.3 & 0.27 & 0.20 & 3.9 & 0.10 & 34.5 & 2.8 \\
$44-149$ & 0.11 & 0.42 & 0.08 & 16.4 & 0.19 & 0.11 & 1.0 & 0.08 & 50.9 & 4.0 \\
$<44$ & $\mathrm{ND}$ & 0.08 & 0.17 & 14.3 & 0.15 & 0.06 & 0.45 & 0.05 & 62.8 & 4.2 \\
\hline
\end{tabular}

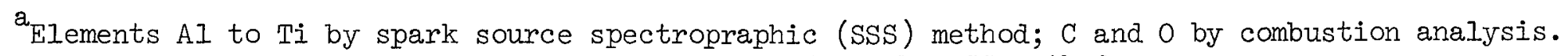
Elements $\mathrm{Pb} \mathrm{Si}, \mathrm{V}$, and $\mathrm{Zr}$ were sought but not found by SSS method.

$\mathrm{b}_{\mathrm{ND}}$ indicates not detected. 
Since the particles seemed well agglomerated during the sieving procedure, though they may not have been agglomerated while circulating in the primary system, interpretation of these results is quite uncertain.

The total masses of samples D2 and D3, recorded in Table 5.2-7, exclude the large metallic pieces separated from the remaining sample by coarse screening. Figures 5.2-1 and 5.2-2 illustrate magnetic and nonmagnetic material taken from dust sample D2, respectively. The source of this material evidently was the metallic insulation lining the internal surface of the hot duct. The total mass of these large metallic pieces in sample D2 was $17 \mathrm{~g}$.

Table 5.2-7. Mass and relative activity of matallic pieces taken from dust sample D3

\begin{tabular}{|c|c|c|c|}
\hline & & $\begin{array}{c}\text { Mass } \\
(\mathrm{g})\end{array}$ & $\begin{array}{l}\text { Relative activity } \\
(\mathrm{mr} / \mathrm{hr})^{\mathrm{a}}\end{array}$ \\
\hline Magnetic & & 0.96 & 40 \\
\hline Semimagnetic & & 1.17 & 240 \\
\hline \multirow[t]{2}{*}{ Nonmagnetic } & & 1.07 & 3 \\
\hline & Total & $3 \cdot 2$ & \\
\hline
\end{tabular}

a All measurements taken at the same distance from sample.

Figure 5.2-3 shows a total of $3.2 \mathrm{~g}$ of metallic pieces taken from sample D3. These pieces were separated into three groups according to magnetic character, and it was unexpectedly found that the major portion of the emitted radiation was associated with the so-called semimagnetic fraction. These observations are summarized in Table 5.2-7.

\subsection{Density Separation of Sample D3 by Zonal Centrifugation}

A method developed at ORNL ${ }^{*}$ employing a zonal centrifugation technique was used to separate dust sample D3 into four density bands. The

\footnotetext{
* Principal developers are T. Tamura and C. Francis of the Environmental Sciences Division. Tests on D3 were performed by $\mathrm{L}$. D. Hulett of the Analytical Chemistry Division.
} 

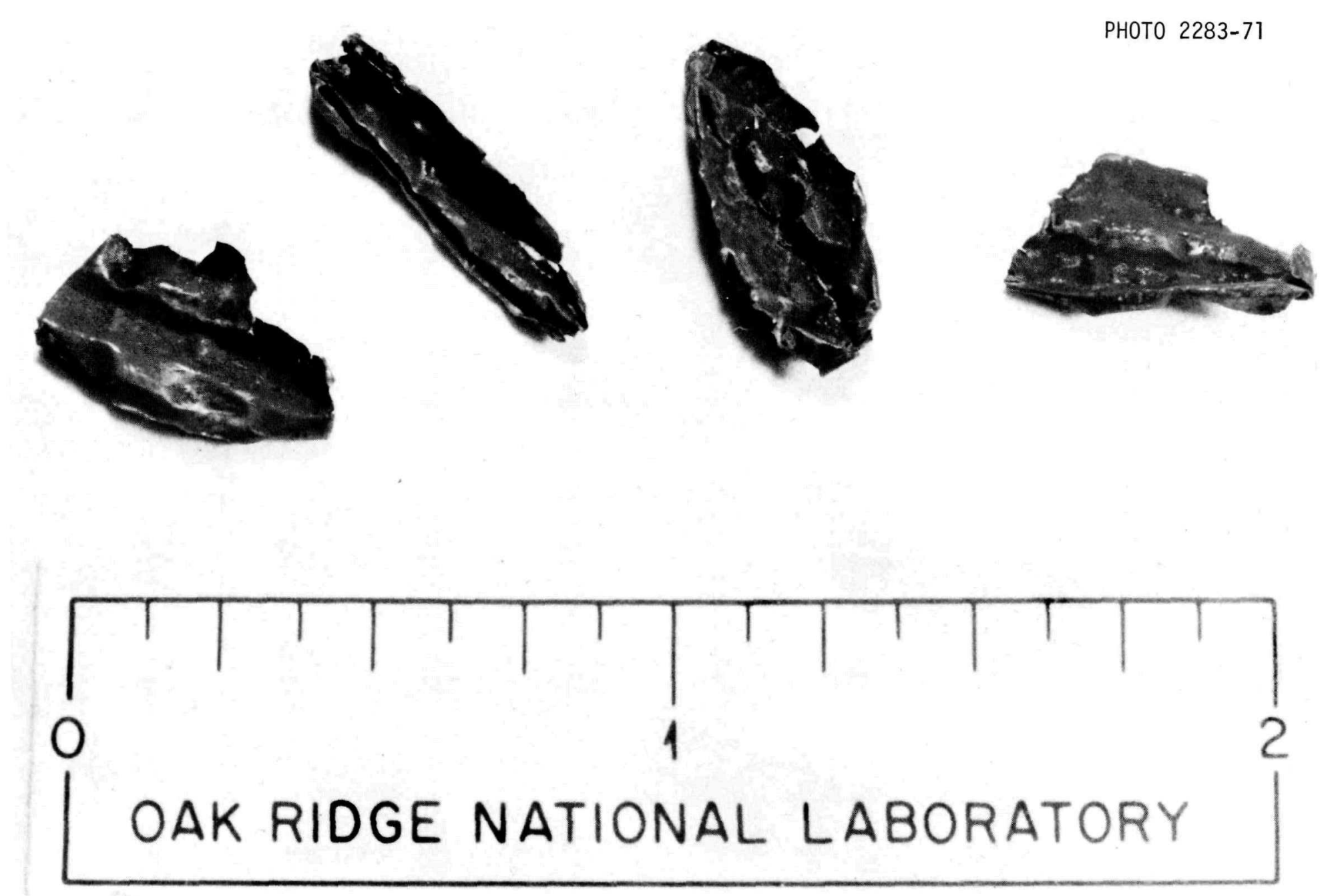

Fig. 5.2-1. Magnetic pieces of hot duct internal insulator material found in dust sample D2. 


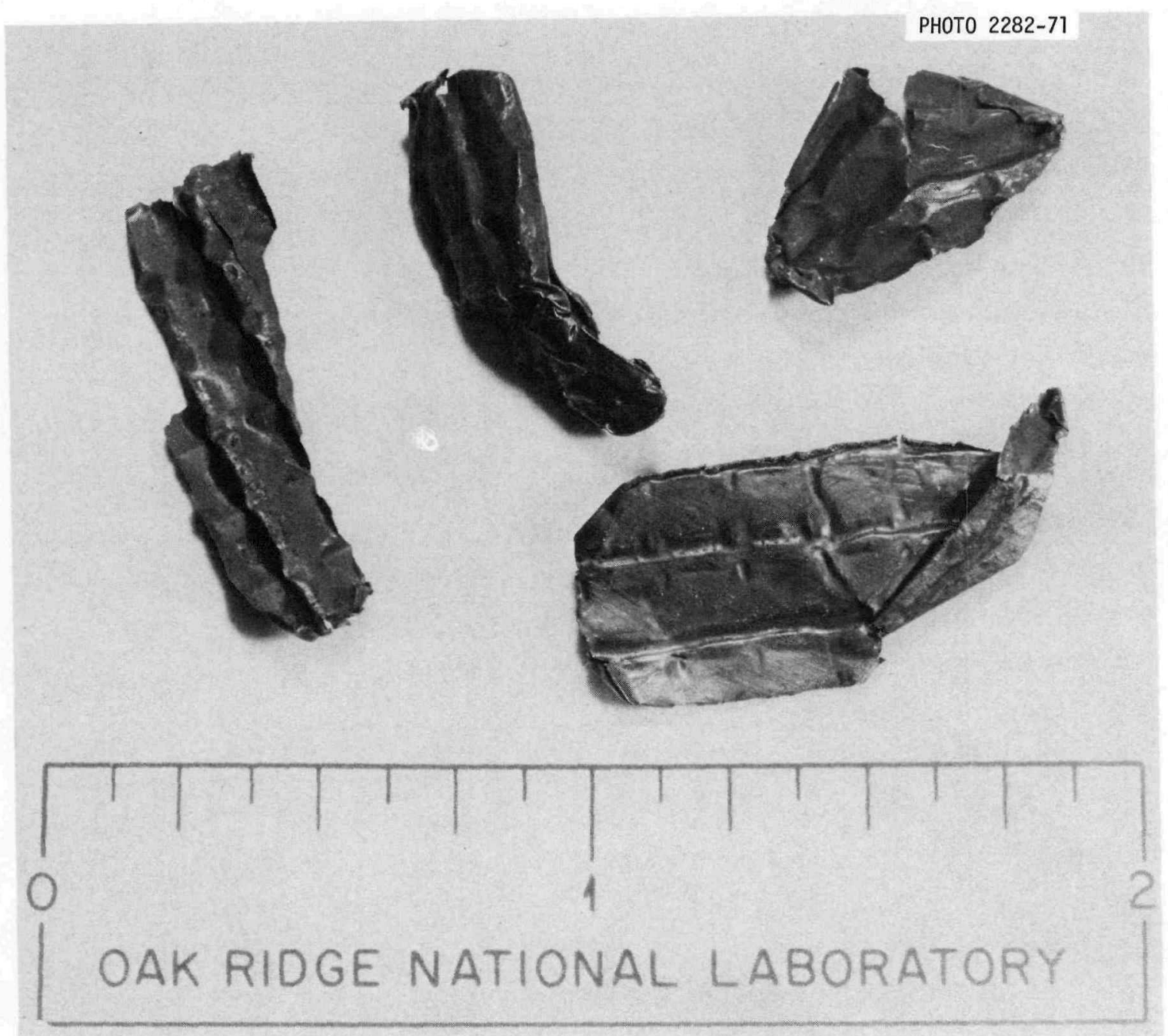

Fig. 5.2-2. Nonmagnetic insulator material found in dust sample D2. 


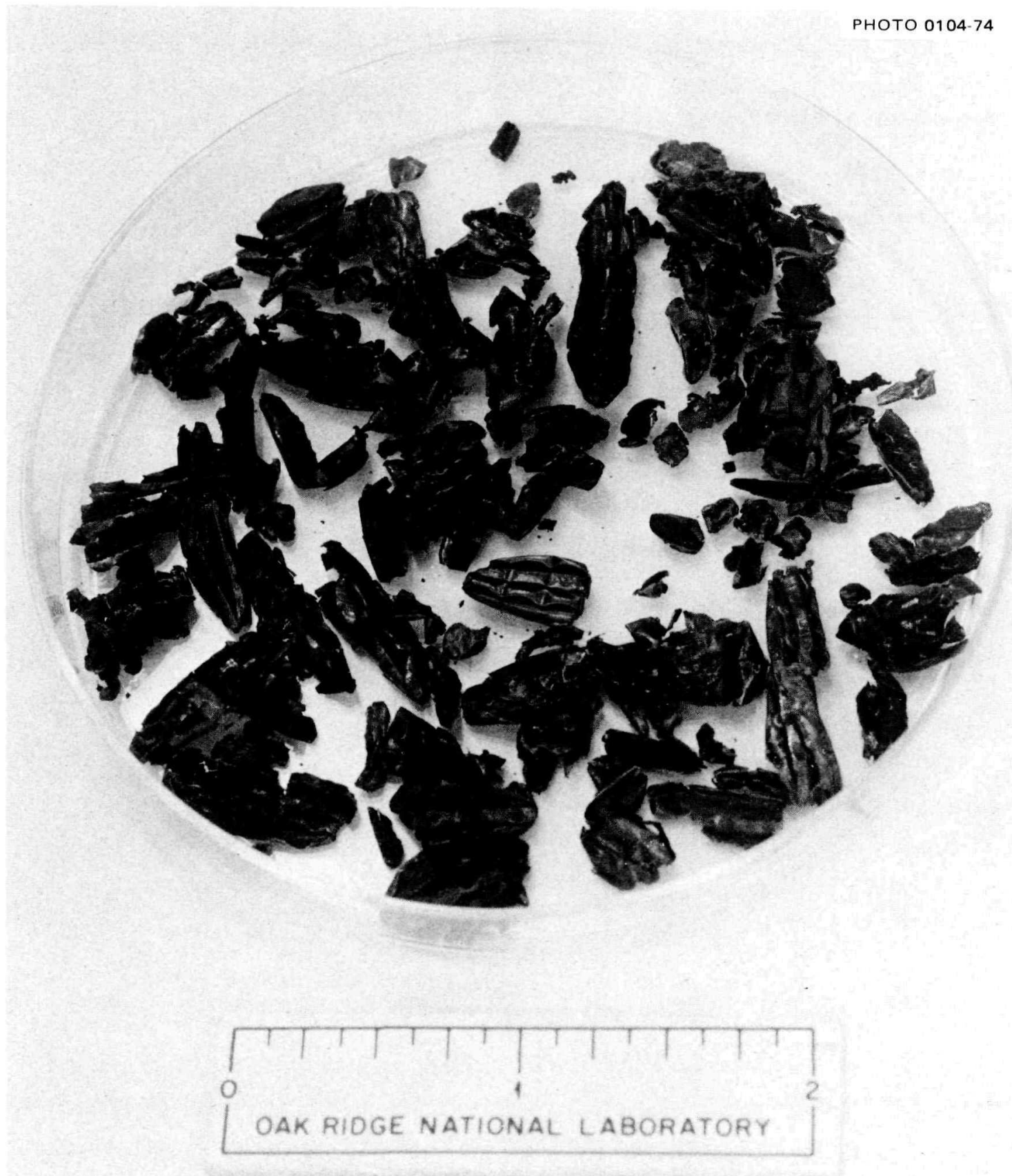

Fig. 5.2-3. Metallic insulator material found in dust sample D3. 
technique first involves the treatment of the sample with ethanol and polyvinylpyrrolidone in a sonic bath to break up the agglomerates into individual particles prior to centrifugation in a variable density gradient solution. This was achieved by controlled injections of tetrabromoethane (TBE) and ethanol into a centrifuge tube such that pure TBE, with a density of $\sim 3 \mathrm{~g} / \mathrm{cm}^{3}$, was present at the bottom of the tube, with a gradual increase in ethanol concentration up to pure ethanol at the top of the tube.

When the treated sample D3 was centrifuged at $2000 \mathrm{rpm}$ using a 12-in. rotor in the above described density gradient, four density bands were achieved with the following qualitative properties.

\subsubsection{Top fraction}

This fraction was the least dense and was collected at the top of the centrifuge tube. The $\mathrm{x}$-ray diffraction (XRD) patterns showed that it contained mostly $\mathrm{SiO}_{2}$ in the form of quartz and cristobalite. The $\mathrm{Fe}_{2} \mathrm{O}_{3}$ and $\mathrm{Fe}_{3} \mathrm{O}_{4}$ were present to the extent of 2 to $10 \%$. Faint lines suggesting $\mathrm{FeSO}_{4} \cdot \mathrm{H}_{2} \mathrm{O}$ and $\mathrm{Sn}$ were seen. The $\mathrm{x}$-ray spectra obtained using the scanning electron micrograph (SEM) confirmed the presence of $\mathrm{Si}$ and $\mathrm{Fe}$, in agreement with the XRD patterns. Significant quantities of $\mathrm{Al}$, $\mathrm{S}, \mathrm{Cl}$, and $\mathrm{Ca}$ were also found. An SEM examination showed that the particle sizes vary greatly. Also, many filament-type particles were found; . in fact, all filamentary particles were concentrated in this fraction, and none were found in the other fractions. An iron map showed that iron had segregated into a separate phase in some cases but also tended to be uniformly dispersed in the $\mathrm{SiO}_{2}$ phase. Aluminum had a similar behavior. The chemical state of the aluminum was not determined in the XRD study. No diffraction lines corresponding to aluminum compounds were seen. A Si map showed that almost all particles in this fraction contained silicon. 


\section{3 .2 Second fraction}

This fraction of the sample was collected about midway down the centrifuge tube. The XRD pattern showed that it was a mixture of elemental iron and iron monocarbide $(\mathrm{FeC})$. Other elements were present, as shown by SEM X-ray spectra, but corresponding compounds were not found.

The SEM photos showed that the particles were generally round and more uniform in shape than the $\mathrm{SiO}_{2}$ particles in the top fraction. An iron map showed that iron was present in almost all particles. The aluminum map showed that aluminum was uniformly distributed in the iron phase.

\subsubsection{Third fraction}

This fraction was collected about three-fourths of the way down the tube. The main element (90\%) was iron, as shown in the SEM x-ray spectrum. Small amounts of $A I$ and $S$ were also present. The $S$ and $A I$ maps showed that these elements are uniformly distributed in the iron phase. The XRD pattern of this fraction could not be identified. The lines were not catalogued in the ASTM tables.

\section{3 .4 Fourth fraction}

This fraction was the most dense of all and settled at the bottom of the centrifuge tube. The x-ray diffraction pattern showed mostly $\mathrm{Fe}_{3} \mathrm{C}$ with smaller amounts of $\mathrm{Cr}_{2} \mathrm{O}_{3}$. Faint lines that corresponded to complex silicates $(\mathrm{Mn}, \mathrm{Ca})_{3}(\mathrm{FeAl})_{2}\left(\mathrm{SiO}_{4}\right)_{4}$ were also found.

Iron was the major element found. The elements Al, Si, S, Ca, Cr, $\mathrm{Mn}$, and $\mathrm{Cu}$ were found in dilute concentrations. The iron map showed that iron was the major constituent of almost every particle. The $\mathrm{S}, \mathrm{Ti}, \mathrm{Cr}$, and $\mathrm{Mn}$ maps showed these elements to be homogeneously distributed in the iron-containing phase. The $\mathrm{Al}, \mathrm{Ca}$, and $\mathrm{Cu}$ maps showed that these elements were also uniformly distributed in the iron phase, but there were also particles of very high concentration. Some particles were found that had a very high concentration of both aluminum and copper. 
5.4 Scanning Electron Microscope (SEM) Photographs of Particles in Samples D3 and D4 Size Fractions*

In the SEM method for analysis of dust particles, a target sample is scanned by a focused beam of electrons, which (among other effects) causes secondary electron emissions from the sample as well as low-energy $x$ rays in the range 0 to $20 \mathrm{keV}$. The examination technique based on this method has a number of distinct advantages in assisting in the determination of the nature of the dust particles. First, the $x$-ray spectrum may be analyzed to determine the elemental composition of the emitting material. In the setup used for these tests, the $\mathrm{x}$ rays were resolved into 1024 channels, each with a resolution of $170 \mathrm{eV}$. Elements equal to and above $\mathrm{Mg}$ in atomic number emit $x$ rays that may thereby be identified by resolution of the spectrum.

A second advantage afforded is the ability to form clear photographs from the secondary electron emissions. Magnification of 150,000X may be achieved with field depths $\sim 100$ times greater than magnifications attainable with optical methods. In addition, the scanning electron beam, which is focused to a diameter of about $100 \AA$, may be stopped at a location of particular interest, so that the elemental composition of one particle or of a portion of one particle may be determined.

The sequence of Figs. 5.4-1 through 5.4-9 illustrate characteristic types of particles observed in the size fractionated portions of sample D3, proceeding from the largest to the smallest size fractions. Figure 5.4-1 shows a particle from the $>420-\mu \mathrm{m}$ size fraction which predominantly emitted $x$ rays associated with $F$, and hence, may be iron oxide or iron carbide. The left photograph, which presents a magnification of 500x, shows the individual subparticles composing the agglomerate to be in the range 10 to $30 \mu \mathrm{m}$. [A later figure (Fig. 5.4-7), taken from $<44 \mu \mathrm{m}$ size classification, evidently shows these same subparticles as separate distinct particles.] The photograph at right in Fig. 5.4-1 shows the entire agglomerate at 100X magnification to be $\sim 0.6 \mathrm{x} 2 \mathrm{~mm}$ in size. The agglomerate appears to have formed a strongly bonded, distinct particle and does not have the appearance of a loose collection formed during the

*The analyses discussed in this section were performed by R. S. Crouse of the Metals and Ceramics Division. 

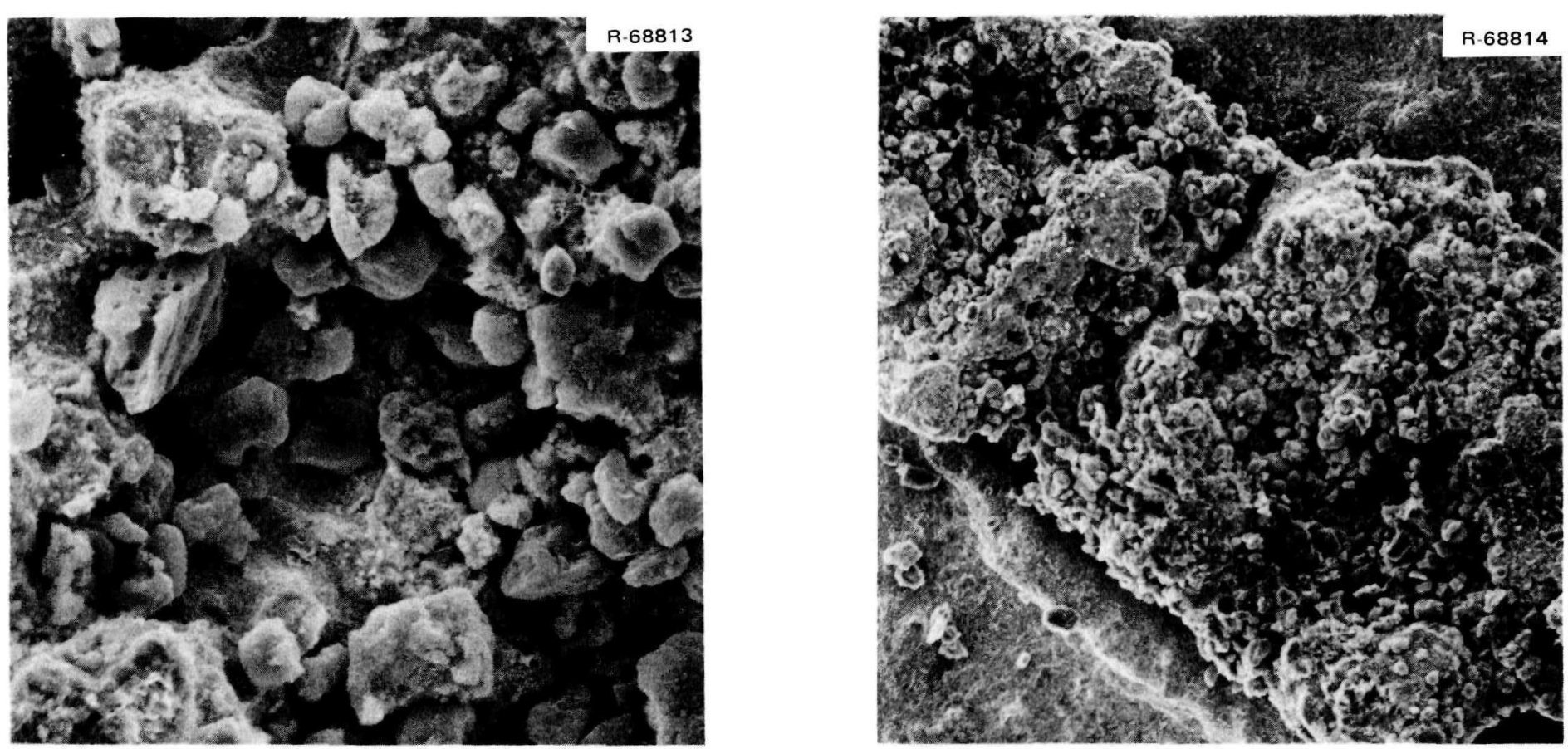

Fig. 5.4-1. SEM micrograph of Fe-rich particle in $>420-\mu \mathrm{m}$ size fraction of D3 dust sample. Left: 500X; right: 1000X magnification. 

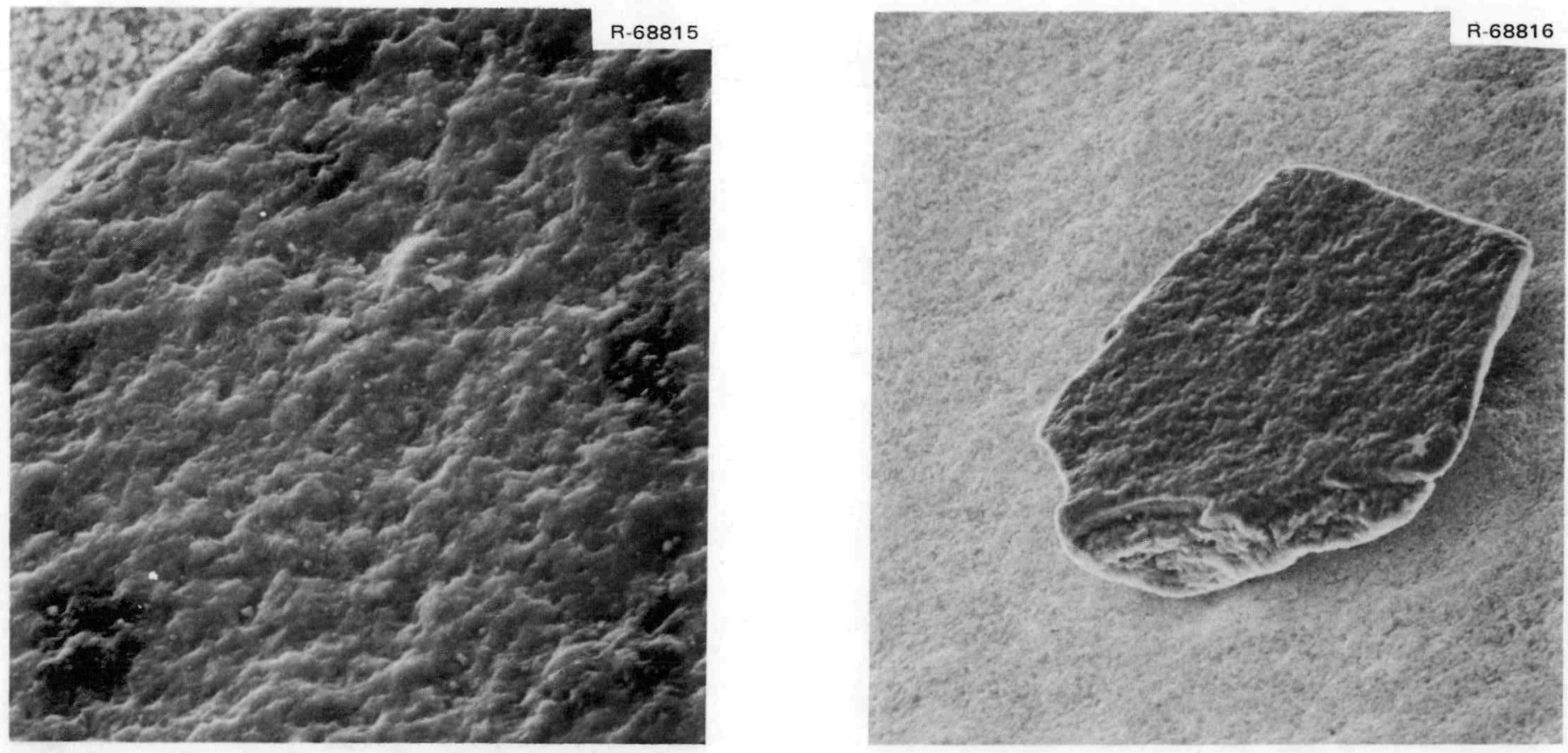

Fig. 5.4-2. SFM micrograph of Fe-rich particle in the 250 to $420-\mu \mathrm{m}$ size fraction of sample D3. Left: 300X; right: 100X magnification. 

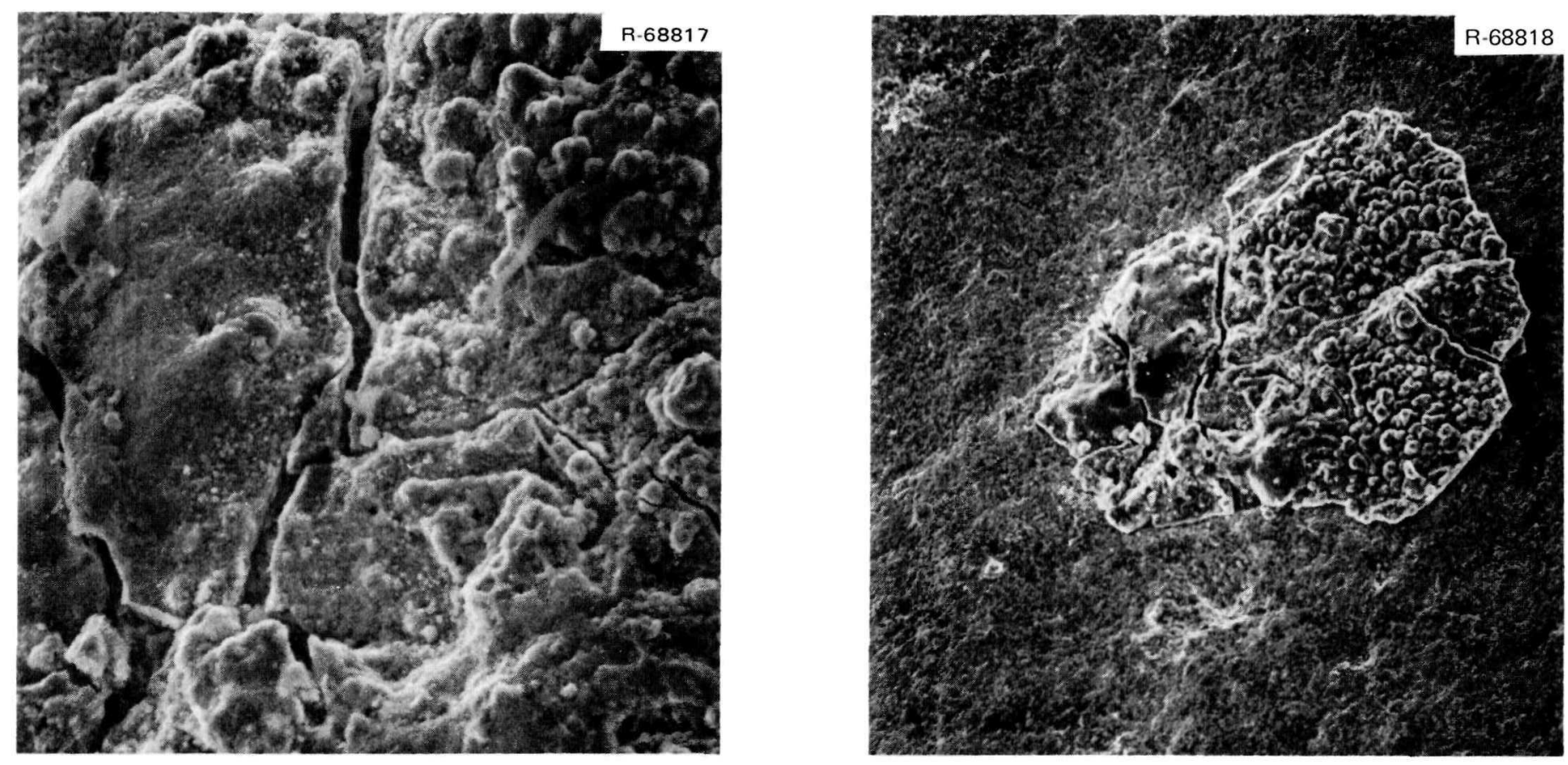

Fig. 5.4-3. SEM micrograph of particle containing $\mathrm{K}, \mathrm{Cr}, \mathrm{Mn}$, and $\mathrm{Fe}$ from 250 to $420-\mu \mathrm{m}$ size fraction of sample D3. Left: 300X; right: 100X magnification. 

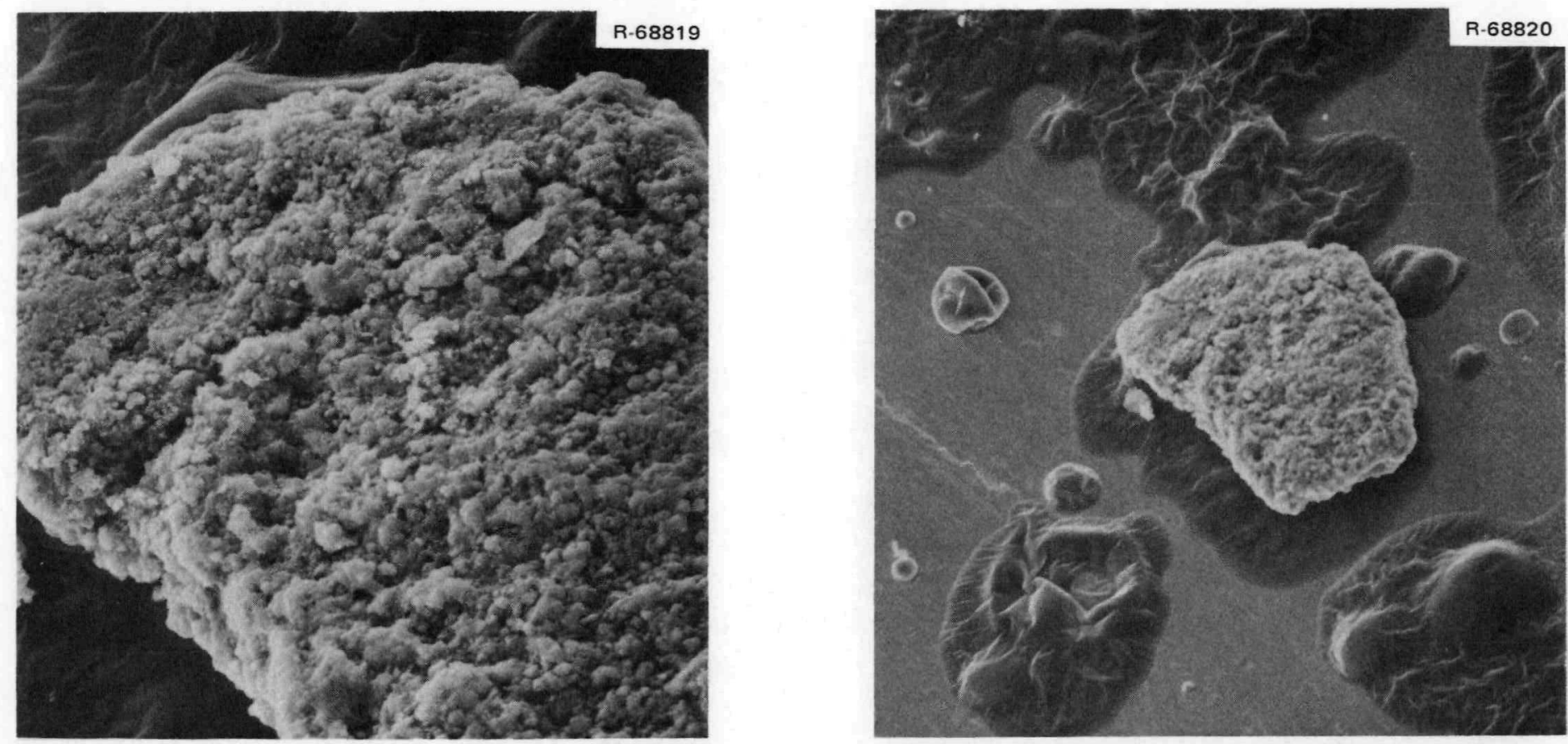

Fig. 5.4-4. SEM micrograph of Fe-rich particle from 175 to 250- $\mu \mathrm{m}$ size fraction of sample D3. Left: 300X; right: 100X magnification. 


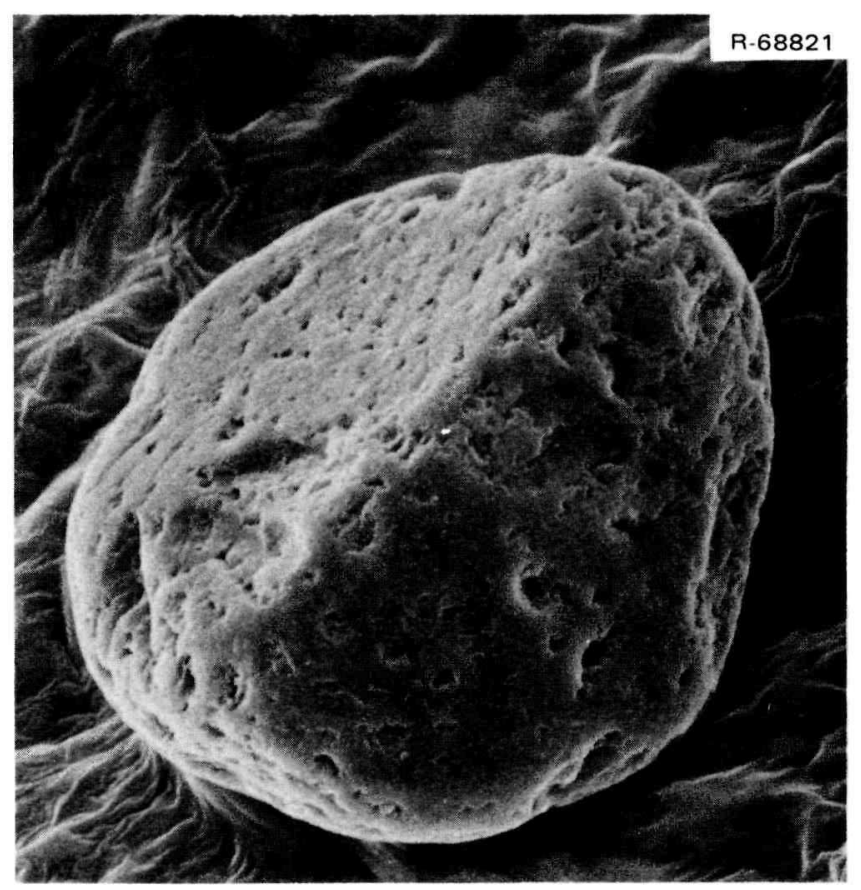

Fig. 5.4-5. SEM micrograph of particle from 149 to $177-\mu \mathrm{m}$ size fraction in D3 sample; magnification = 300X (probably graphite).

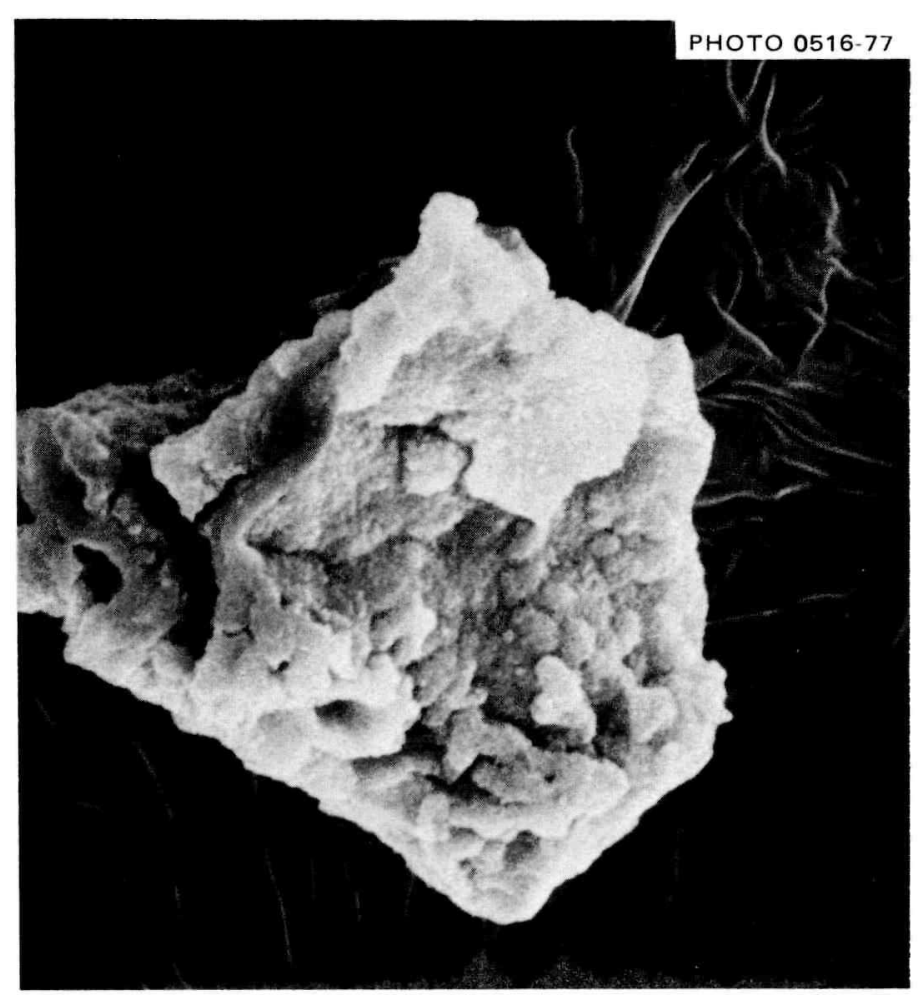

Fig. 5.4-6. SEM micrograph of Fe-rich particle from 44 to 149- $\mu \mathrm{m}$ fraction of sample D3; magnification = 1000X. 

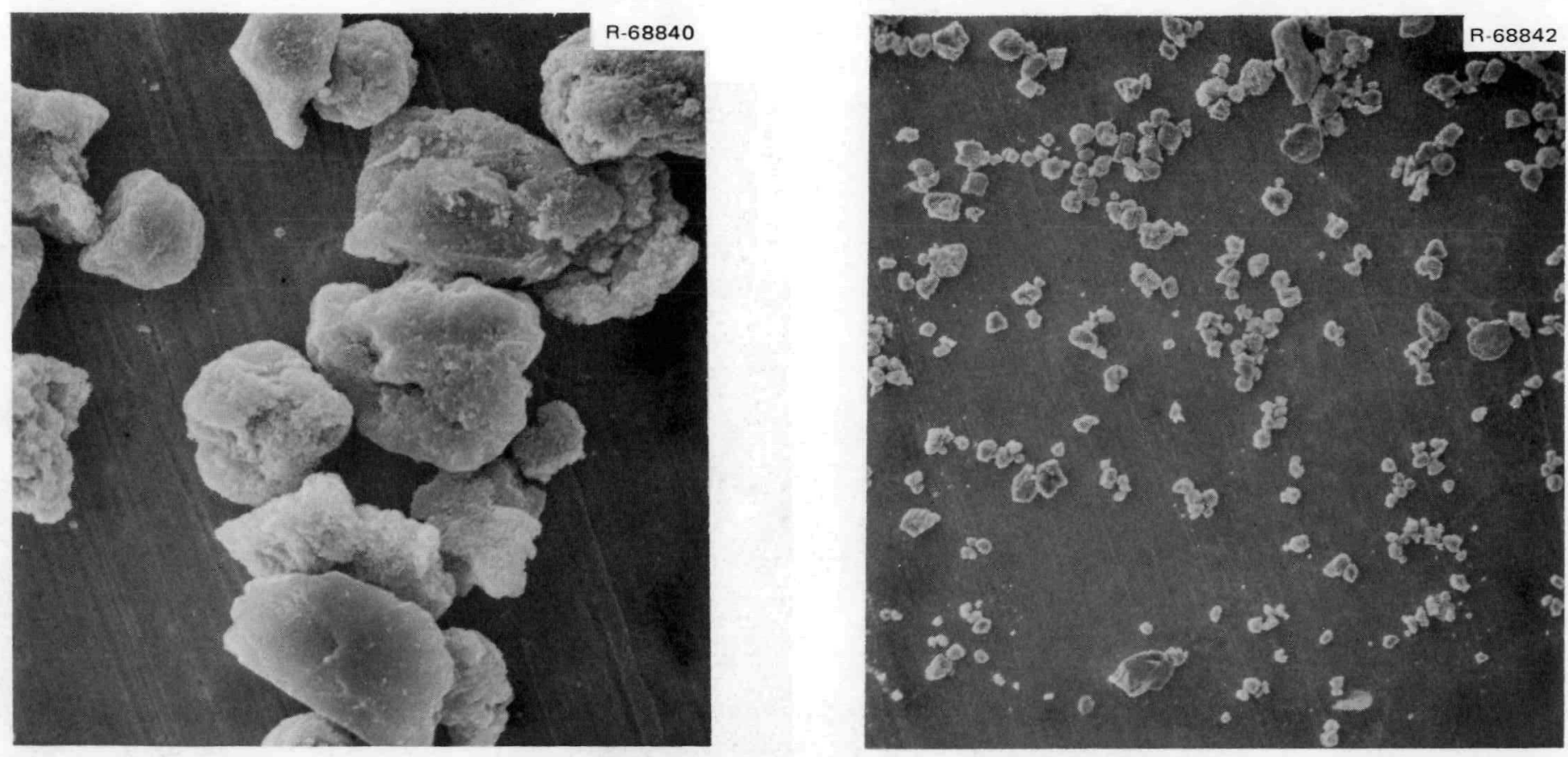

Fig. 5.4-7. SEM micrograph of Fe-rich particles in the 10 to $40-\mu \mathrm{m}$ size fraction of sample D3. Left: 1000X; right, l00X magnification. 


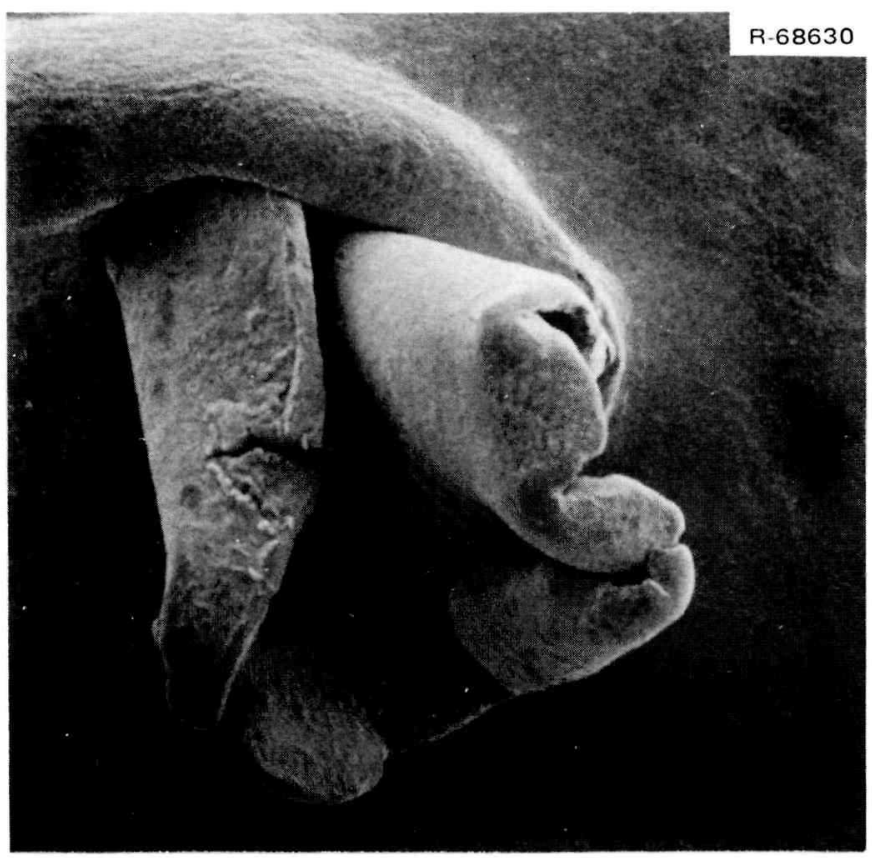

Fig. 5.4-8. SEM micrograph from $>420-\mu \mathrm{m}$ size fraction of $\mathrm{D} 4$ sample, magnification $=40 \mathrm{X}$. 

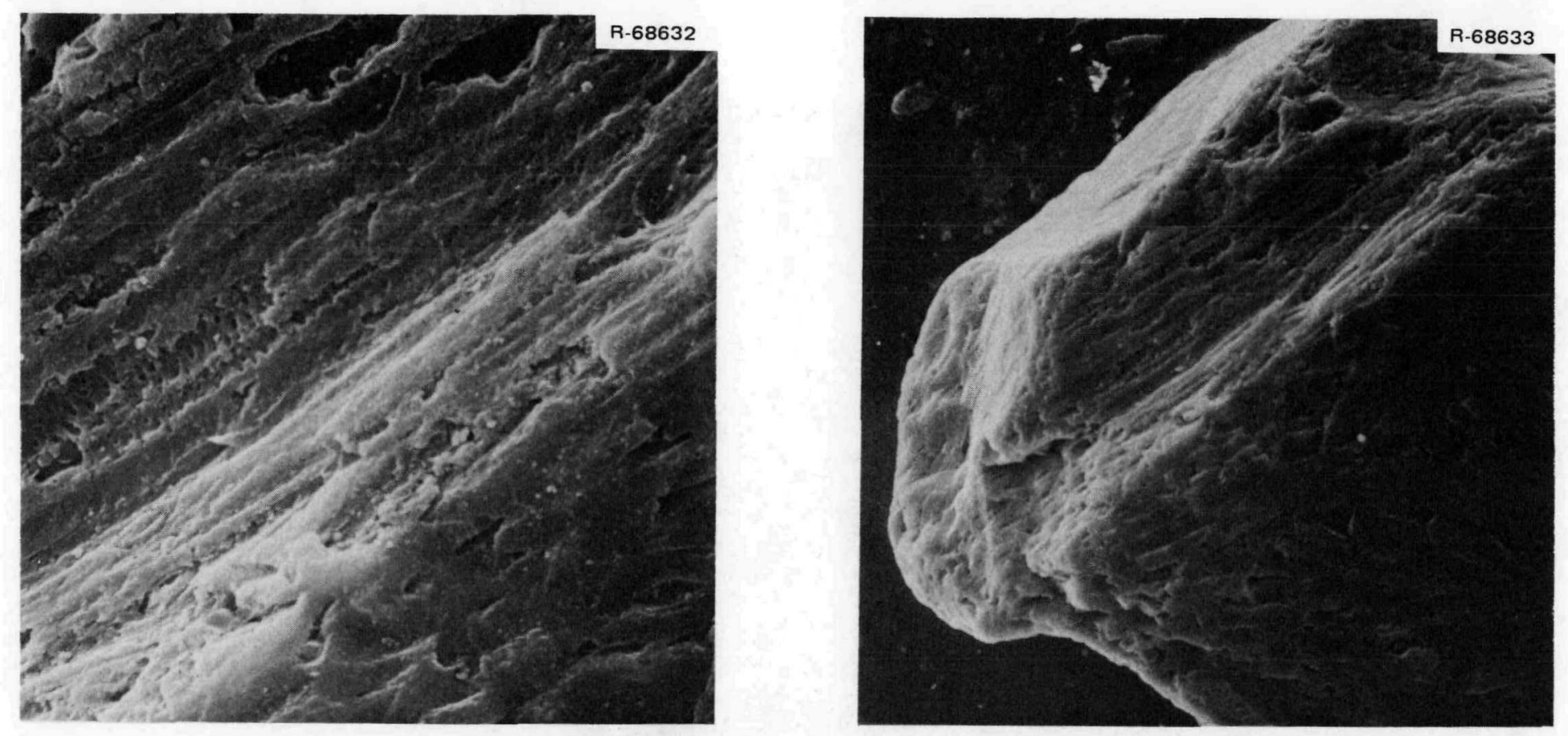

Fig. 5.4-9. SEM micrograph from 250 to $420-\mu \mathrm{m}$ size fraction of sample D4, evidently graphite. Left: 600X; right: 175X magnification. 
process of collecting and storing the sample. Hence, apparently both large agglomerates, in the size range 0.6 to $2 \mathrm{~mm}$, and smaller component particles, in the size range 10 to $30 \mu$, circulated with the primary coolant.

Figures 5.4-2 and 5.4-3 show two particles from the 250 to $420-\mu \mathrm{m}$ size fraction. The first of these shows an Fe-rich particle $\sim 250 \mu \mathrm{m}$ in size, which may have been a rust flake. The smooth surface may be indicative of particulate surface removed from a smooth iron substrate. Figure 5.4-3 shows a particle about the same size as the particles shown in Fig. 5.4-2 and containing $\mathrm{K}, \mathrm{Cr}, \mathrm{Mn}$, and $\mathrm{Fe}$.

Figure 5.4-4 shows an Fe-rich particle from the 175 to $250-\mu \mathrm{m}$ size fraction, which appears to be similar to that shown in Fig. 5.4-1. This, evidently, is a common type of iron-rich particle which was found in most of the size fractions. Again, this particle appears to be a strongly adherent agglomerate of particles in the 10 to $50-\mu$ range.

Figure 5.4-5 shows another common type of particle, which in this case was taken from the 149 to $177-\mu \mathrm{m}$ size fraction. No $\mathrm{x}$-ray emission lines were observed from this particle, and hence, it is believed to be graphite. (As noted earlier, this procedure does not detect $\mathrm{x}$ rays from elements below $\mathrm{Mg}$ in atomic number.)

Figures 5.4-6 and 5.4-7 show the smaller sized iron-rich particles that, evidently, make up the larger agglomerates seen in the earlier figures. In particular, the particle sizes seen in Fig. 5.4-7 range from $\sim 10$ to $40 \mu \mathrm{m}$ and show a quite similar appearance to the subparticles composing the agglomerate shown in Fig. 5.4-1. Evidently, particles such as these circulated both as individual particles in the 10 to $40-\mu$ size range, as well as agglomerates of up to $2 \mathrm{~mm}$ in size.

The figures 5.4-8 and 5.4-9 show a pair of SEM micrographs taken from size fractions of dust sample D4. The first in this sequence, Fig. 5.4-8, shows a metallic particle $\sim 2 \mathrm{~mm}$ in diameter, which is evidently from the same source as those shown in Figs. 5.2-1 to 5.2-3. Most particles like this were removed from the sample prior to further analyses. 
Figure 5.4-9 shows two magnifications of a particle $\sim 500 \mu \mathrm{m}$ in diameter, which is evidently composed of graphite. The characteristic layering and porous structure of graphite are clearly visible. No x-ray emissions were observed.

Figure 5.4-10, taken from the 250 to 420- $\mu \mathrm{m}$ size fraction, shows two types of particles; the bottom one, exhibiting only $\mathrm{Fe} x$-ray emissions, appears to be an agglomerate composed of much smaller, compacted iron particles. The upper particle, indicating a layered structure, exhibited both $\mathrm{Fe}$ and $\mathrm{Cr} \mathrm{x}$-ray emissions.

A particle from the 149 to $177-\mu \mathrm{m}$ size fraction showing Si $x-r a y$ emission (evidently silica) is shown in Fig. 5.4-11. Figure 5.4-12, taken from the same fraction, shows a loose agglomeration of two types of particles. The larger particles, which are $\sim 200 \mu \mathrm{m}$ in diameter, exhibited both $\mathrm{Cr}$ and $\mathrm{Fe} \mathrm{x}$-ray emissions. The smaller particles, ranging from 10 to $40 \mathrm{\mu m}$, only exhibited Fe emissions and are evidently the same iron-rich particulates seen in earlier photos. From its appearance in the figure, the agglomeration formed during sample storage is a loose one.

Figure 5.4-13 shows two types of particles taken from the 177 to $250 \mu \mathrm{m}$ size fraction. On the left is a loose agglomerate of the common iron-rich particle in the size range of 10 to $40 \mu \mathrm{m}$. Again, from its appearance, the agglomeration appears to be an effect of sample storage, and the particles in this case most likely circulated individually in the primary circuit. At the right is a typical graphite particle of $\sim 300-\mu \mathrm{m}$ diam showing the typical layered and porous structure.

Figures 5.4-14 through 5.4-16 are taken from the 44 to 149- $\mu \mathrm{m}$ size fraction of sample D4. The first of these shows two magnifications of a $\sim 130-\mu \mathrm{m}$ particle which exhibited $\mathrm{Ca}$, Ti, $\mathrm{Cr}$, and Fe x-ray emissions. Noted again in the left photograph are the common iron-rich particulates in the 10 to $20-\mu \mathrm{m}$ size range, which, evidently, clung to the larger particle

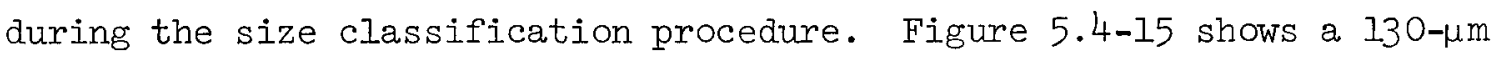
particle that exhibited $\mathrm{Mn}$ and $\mathrm{Si} \mathrm{x}$-ray emissions.

Figure 5.4-16 shows a loose agglomeration of two types of particles; the larger ones, $\sim 150 \mu \mathrm{m}$ in diameter, exhibited $\mathrm{Fe}, \mathrm{Cr}$, and $\mathrm{Ni}$ emissions 


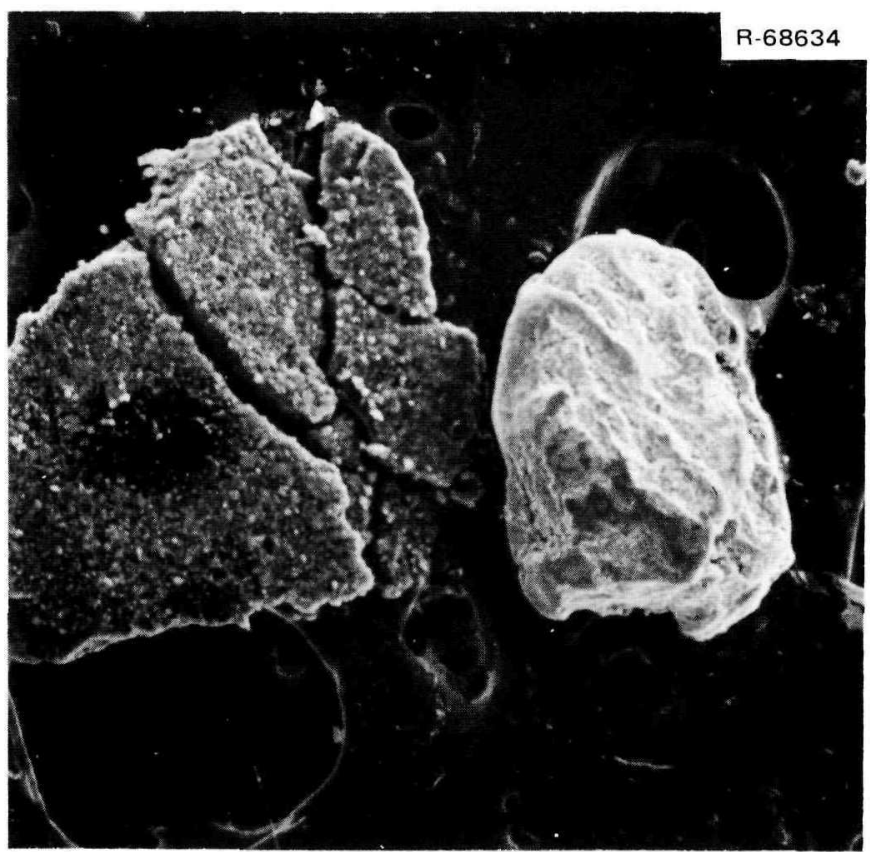

Fig. 5.4-10. SEM micrograph from the 250 to $420-\mu \mathrm{m}$ size fraction of D4 sample. Bottom, Fe-rich; top, contains $\mathrm{Fe}$ and $\mathrm{Cr}$; magnification = 100X. 

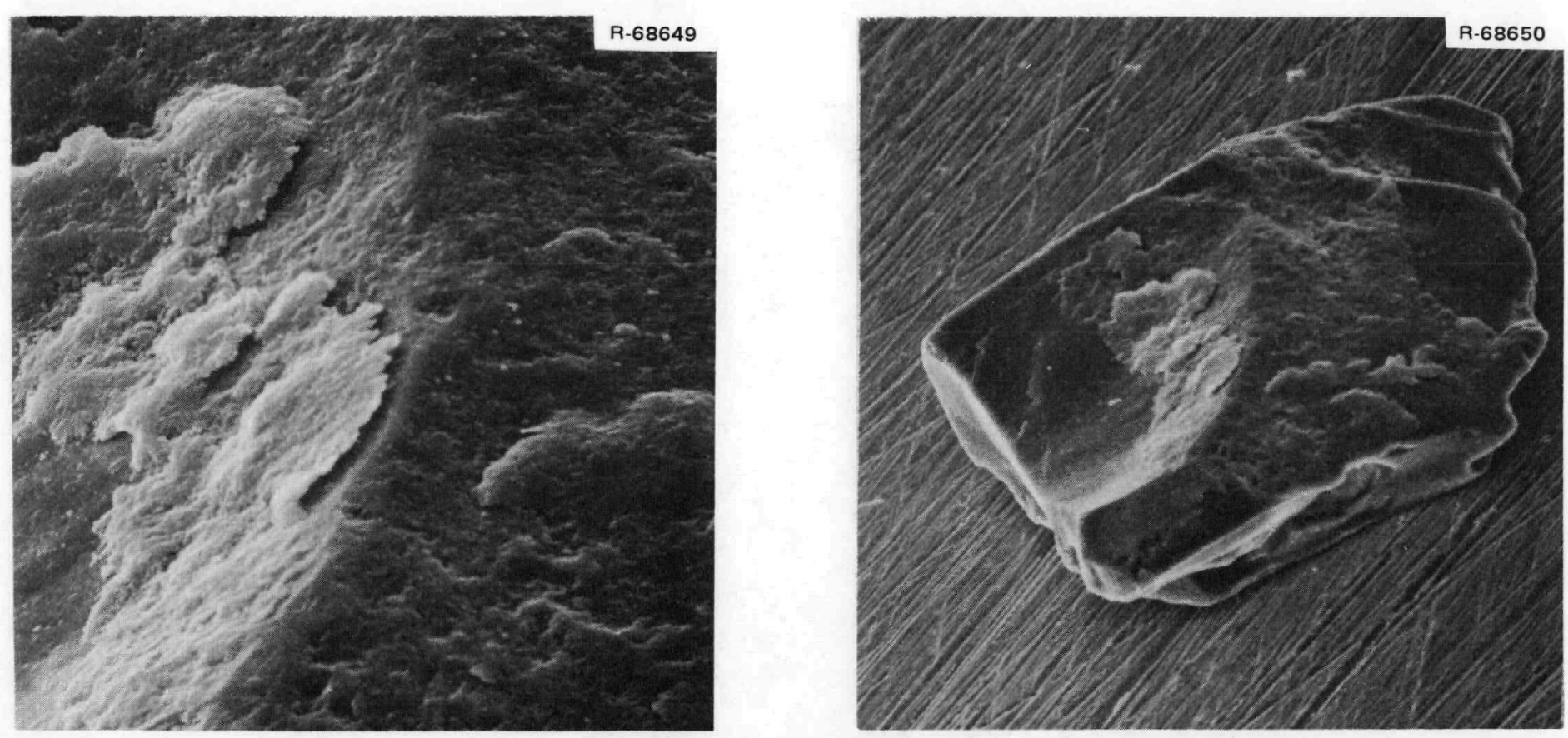

Fig. 5.4-11. SEM micrograph of Si-rich particle from 149 to $177-\mu \mathrm{m}$ size fraction of sample D4. Left: 1000X; right: $300 \mathrm{X}$ magnification. 

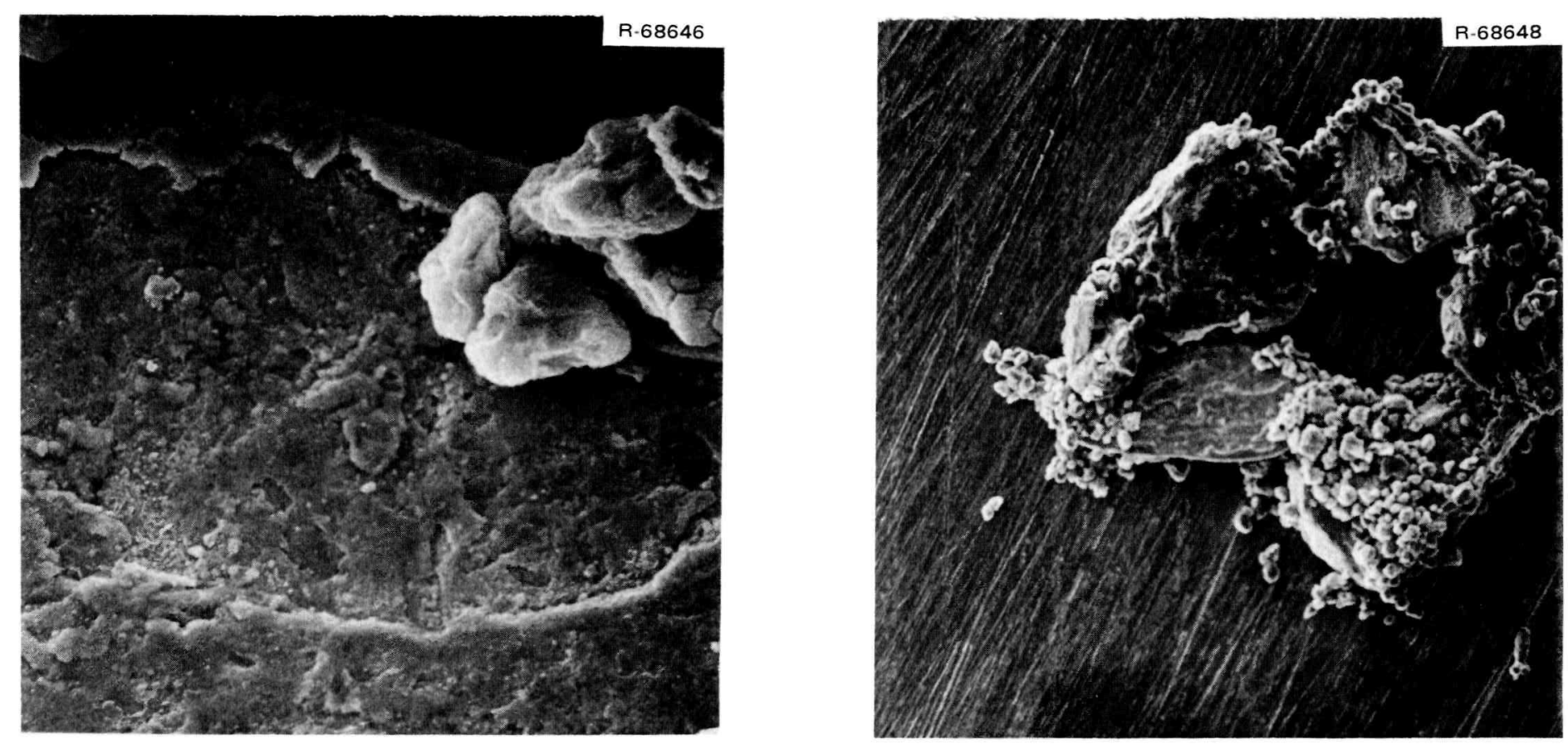

Fig. 5.4-12. SEM micrograph from 149 to $177-\mu \mathrm{m}$ size fraction of D4 dust sample. Large particles show $\mathrm{Cr}$, Fe emissions; small particles show Fe emissions. Left: 1000X; right: 100X magnification. 

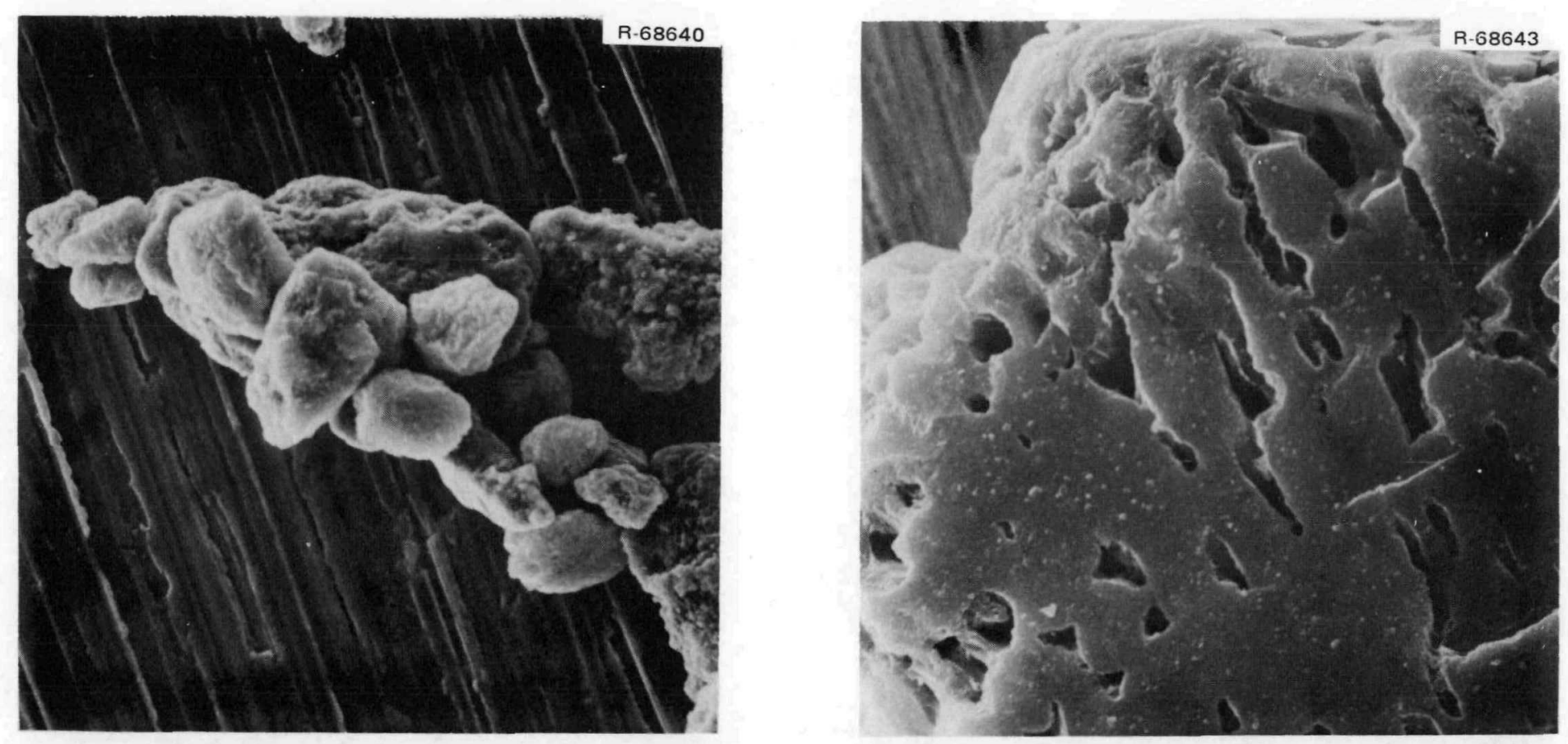

Fig. 5.4-13. SEM micrograph from the 177 to $250-\mu \mathrm{m}$ size fraction of D4 sample. Left, iron-rich particle; right, graphite; magnification $=1000 \mathrm{X}$. 

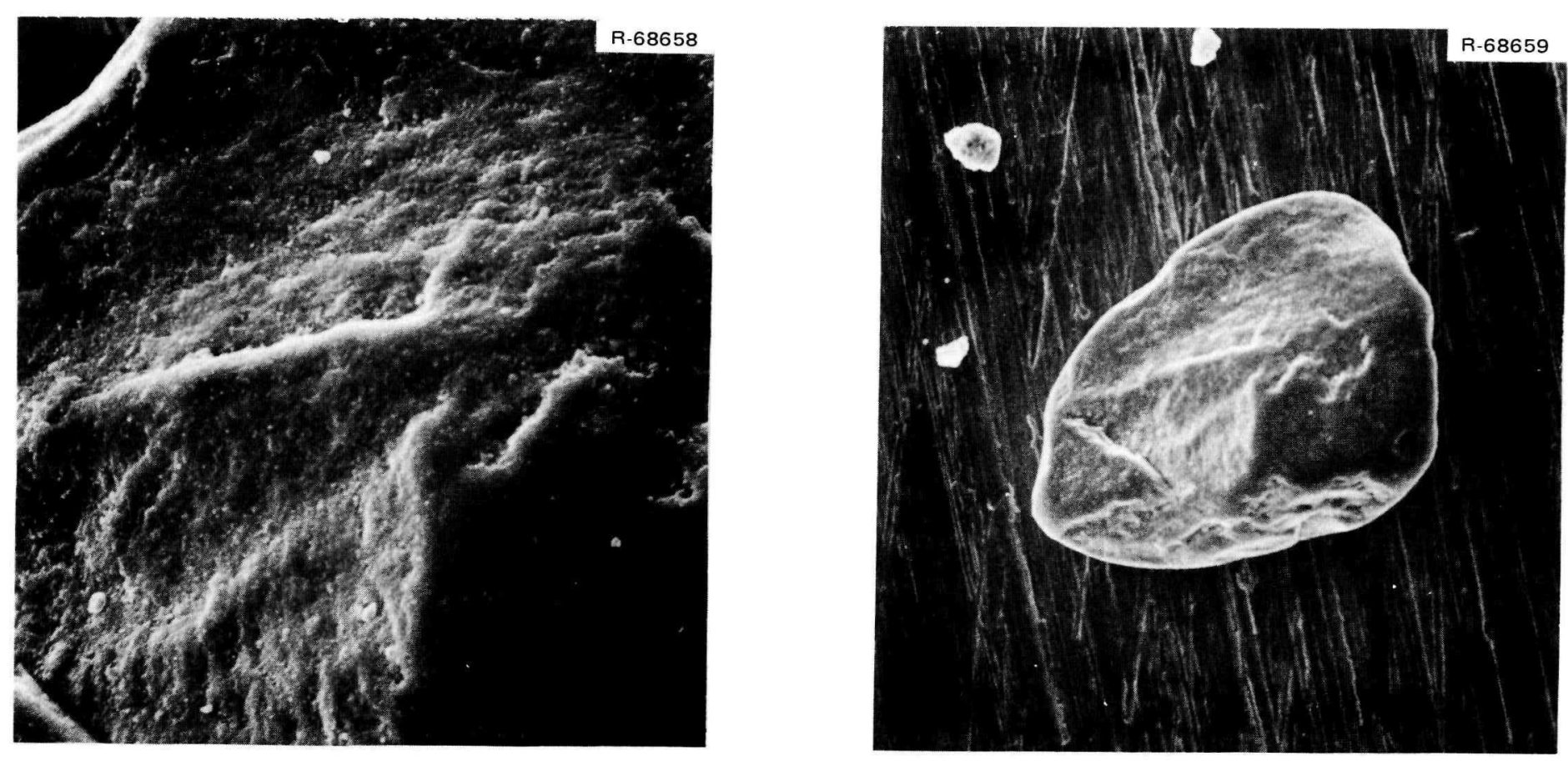

$\underset{⿱ E}{\stackrel{E}{G}}$

Fig. 5.4-14. SEM micrograph from the 44 to 149-um size fraction of sample D4. Content: Ca, Ti, Cr, and Fe. Left: 1000X; right: 300X magnification. 

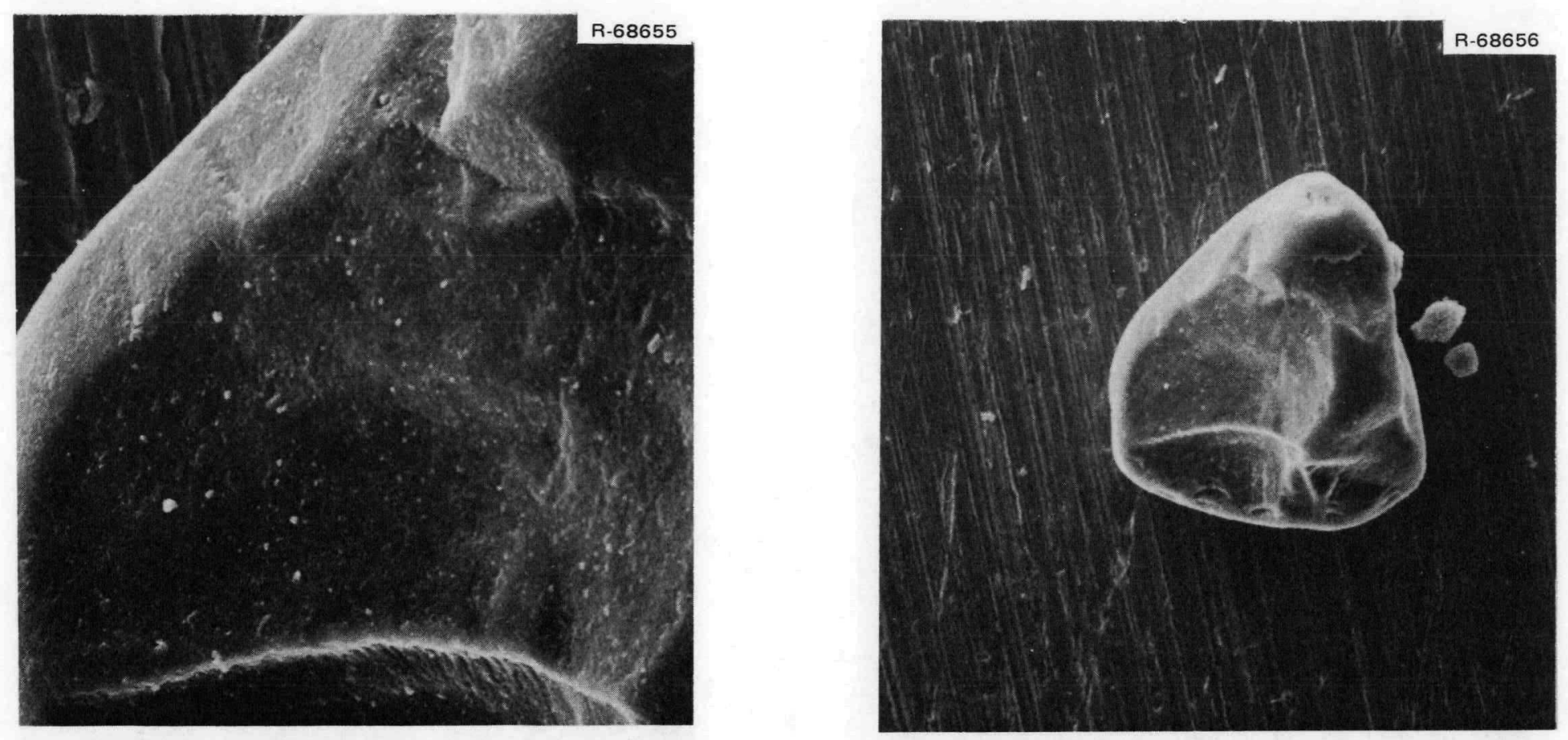

忎

Fig. 5.4-15. SEM micrograph from 44 to 149- $\mu \mathrm{m}$ size fraction of sample D4. Content: Mn and Si. Left: 1000X; right: 300X magnification. 

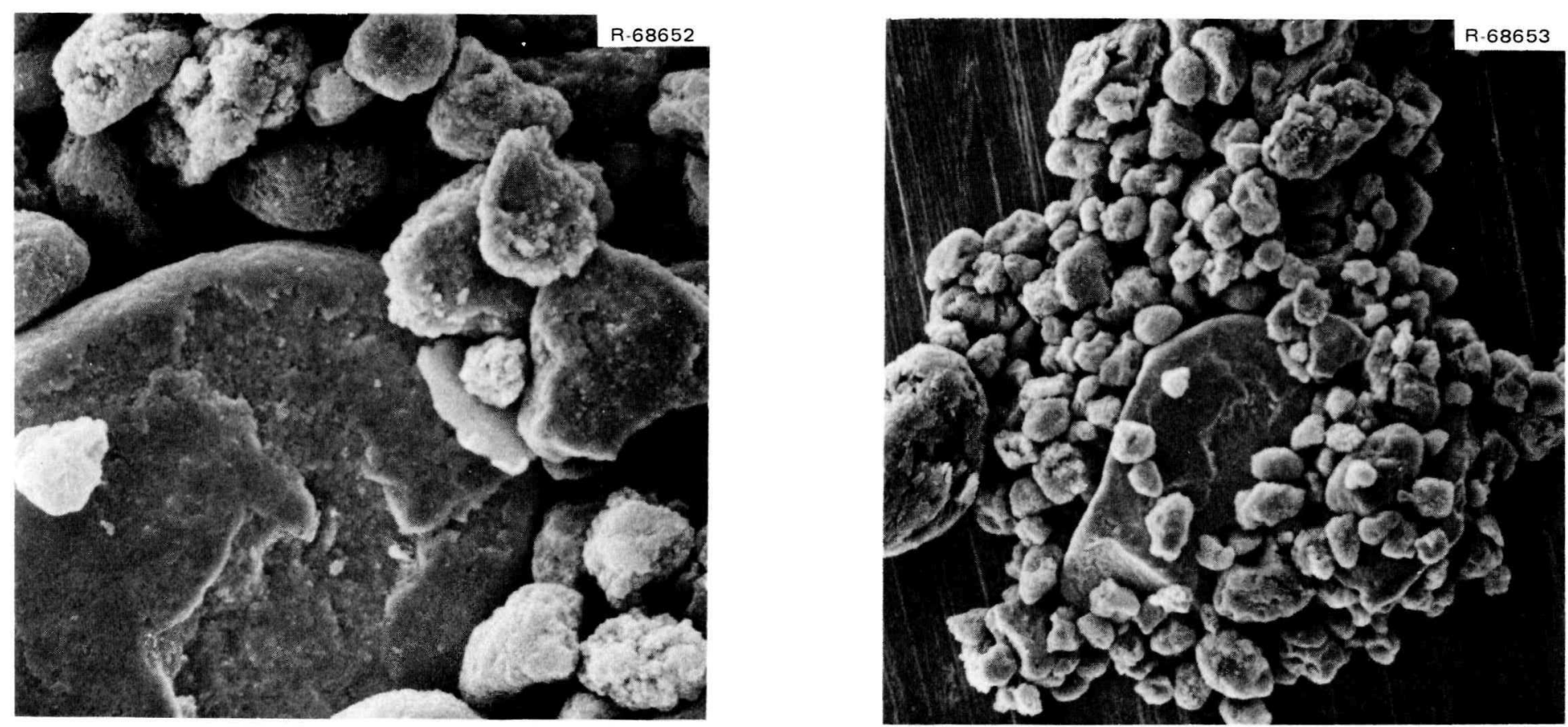

Fig. 5.4-16. SEM micrograph from 44 to $149-\mu \mathrm{m}$ size fraction of sample D4. Large particles are stainless steel; small particles are iron oxide or iron carbide. Left: 1000X; right: 300X magnification. 
characteristic of stainless steel. The smaller particles appear to be the common iron-rich particles held loosely to the larger particle.

Figures 5.4-17 through 5.4-19 are taken from the $<44-\mu \mathrm{m}$ size fraction of dust sample D4. Figure 5.4-17 shows evidently graphite particles in the size range 15 to $50 \mu \mathrm{m}$. These particles exhibited no $x$-ray emission and have the layered appearance of graphite. Figure 5.4-18 shows the common iron-rich particles in the size range of 15 to $40 \mu \mathrm{m}$. Figure 5.4-19

shows two types of particles. The smaller ones, in the size range of 10 to $50 \mu \mathrm{m}$, only exhibit $\mathrm{Fe}$ emissions and are evidently the common iron-rich particles. The large 20 by $100-\mu \mathrm{m}$ particle, shown magnified at left, appears filamentary and exhibited $\mathrm{Si}$ and $\mathrm{Ca}$ emissions exclusivley.

In summary, the SEM micrographs show that: (1) the most frequentIy observed particle is an iron-rich particle (either the oxide or the carbide) in the size range of 10 to $40 \mu \mathrm{m}$, (2) these iron-rich particles frequently appear strongly agglomerated, achieving sizes on the order of a few millimeters, or they may be loosely agglomerated, attaching themselves to larger particles during the size classification procedure, and (3) stainless steel, graphite, and silica particles are also observed.

\subsection{Further Particle Size Distribution Data Emphasizing}

$$
0.1 \text { to } 10 \mu \mathrm{m} \text { Size Range }
$$

The fines of dust samples D3 and D4 were examined by means of a Zeiss Particle Size Analyzer. A small portion of the composite sample was tamped onto an adhesive surface in a fashion that selectively removed some of the larger sized particles from the sample. The field was photographed and scanned optically by the Zeiss system, which mechanically sets a visible size range on the field against which particles may be matched. This rather tedious method is necessary for samples that are not completely de-agglomerated, since operator judgement may be used. to distinguish between touching particles.

Results for samples D3 and D4 are indicated at left in Fig. 5.5-1. Each data point signifies the number of particles counted within the indicated size range. It may be seen that, on a number basis, the fines 

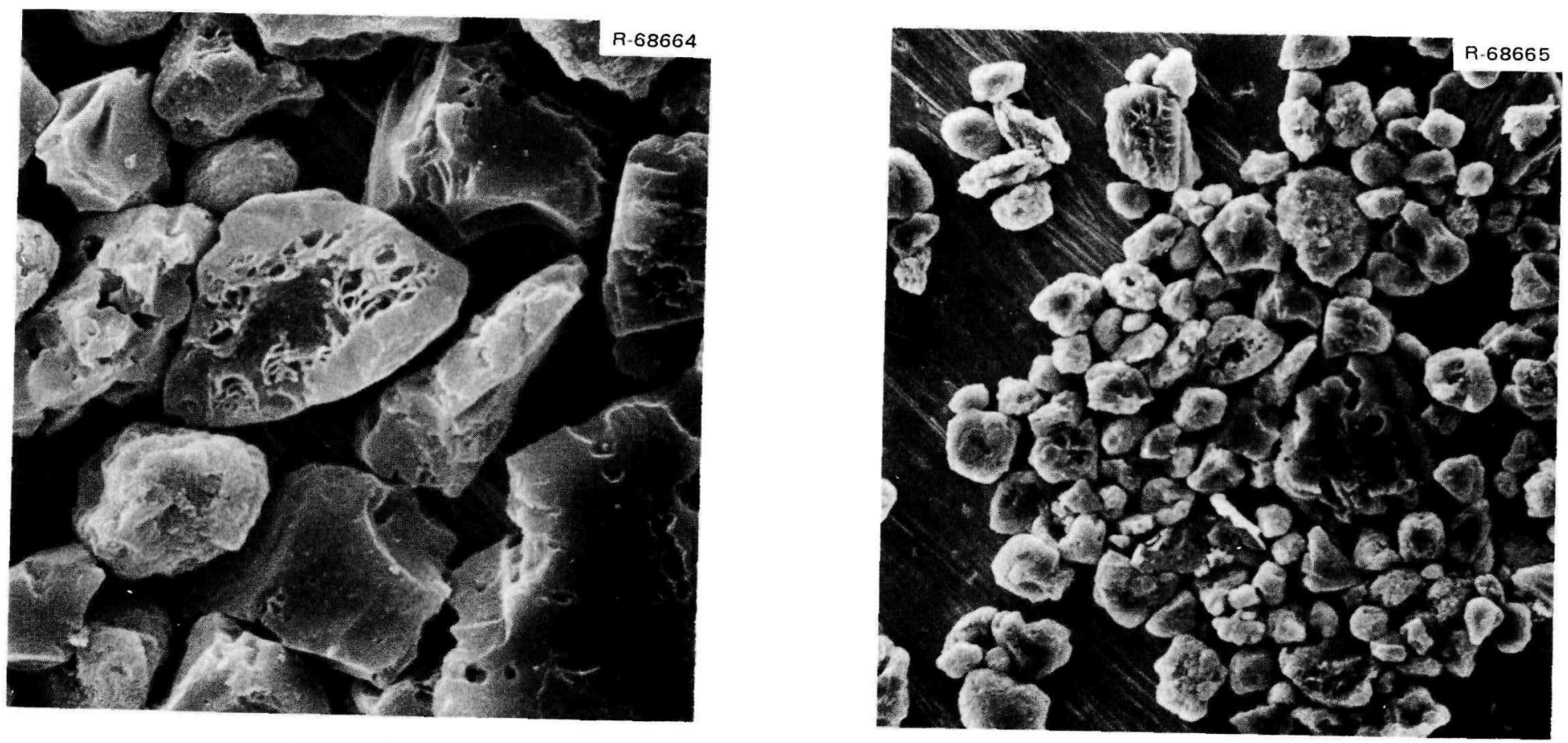

D4, evidently graphite. Left: looox; right: 300X magnification.
3ample 

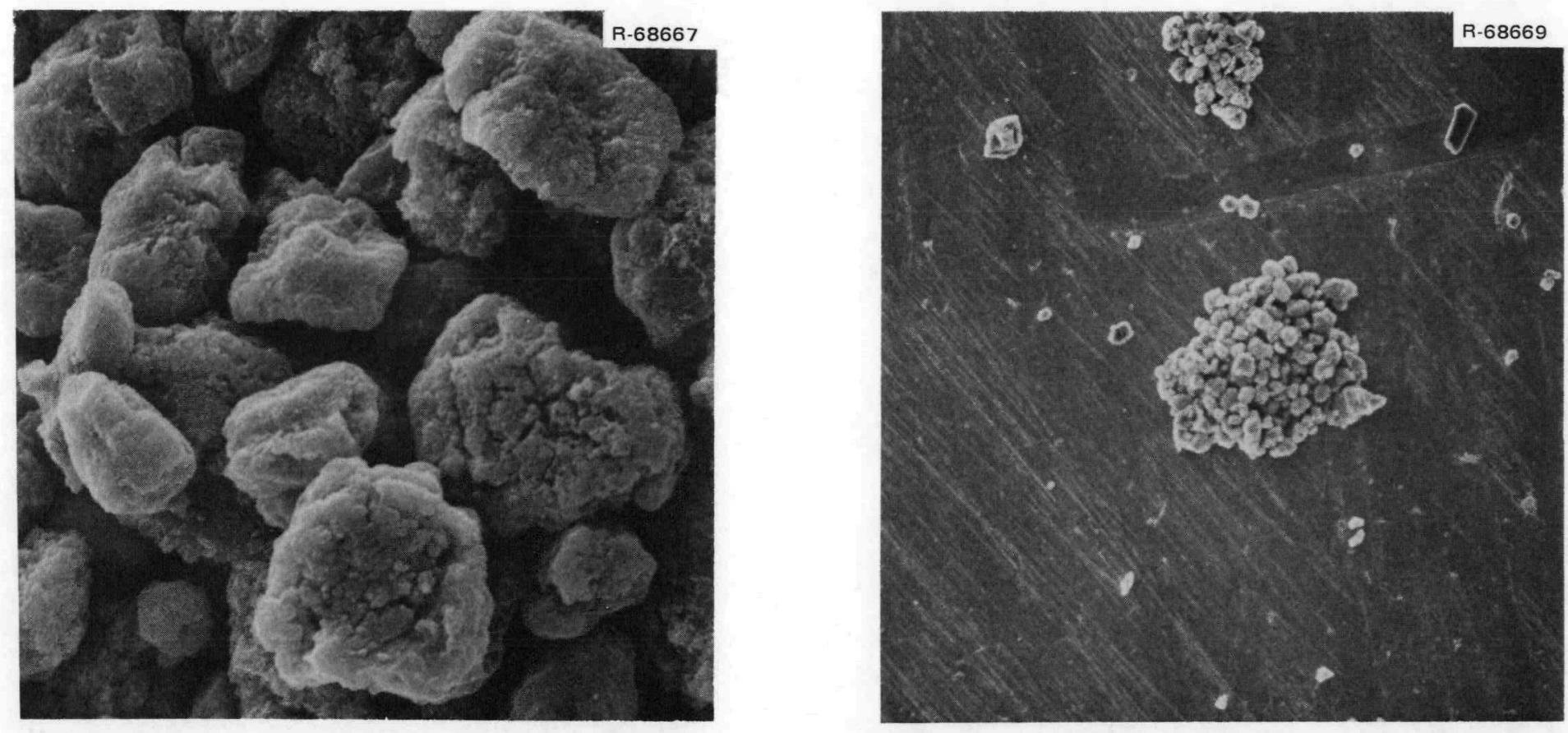

Fig. 5.4-18. SEM micrograph from $<44-\mu \mathrm{m}$ size fraction of sample D4, evidently FeO. Left: 1000X; right: I00X magnification. 

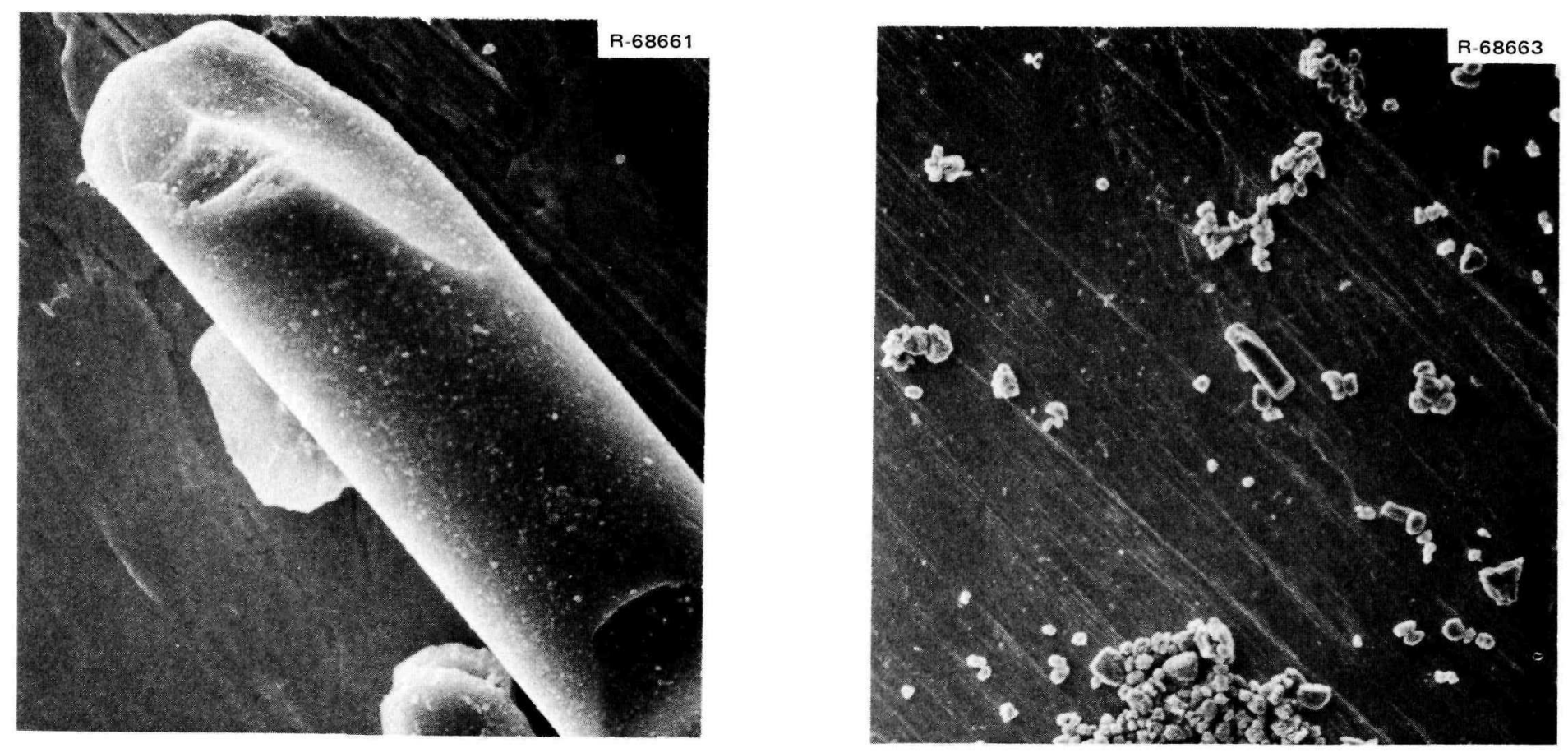

Fig. 5.4-19. SEM micrograph from $<44-\mu m$ size fraction of sample D4. Small particles are FeO, and large particles contain $\mathrm{Ca}$ and Si. Left: 1000X; right: 100X magnification. 
ORNL DWG 76-17513

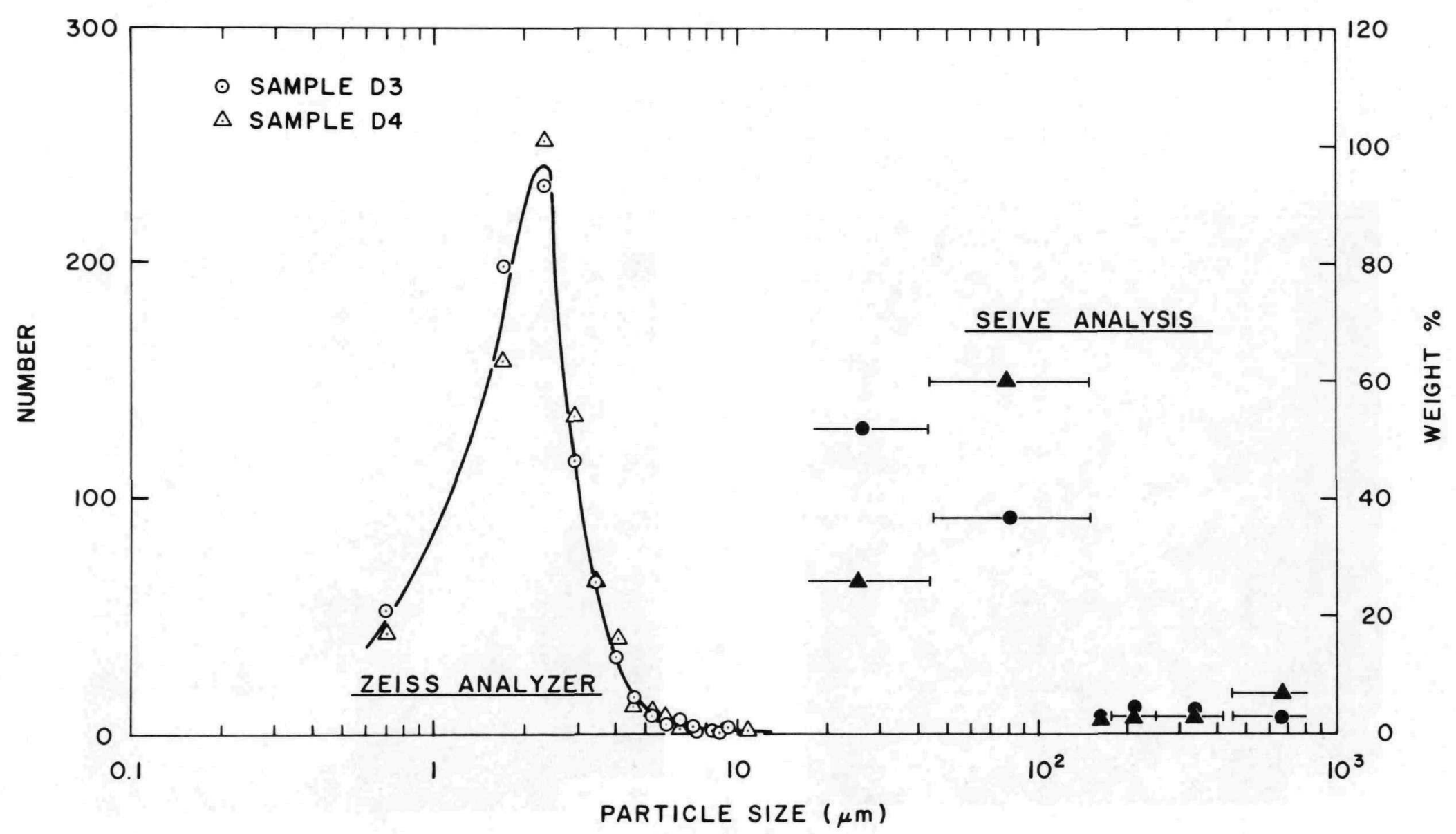

Fig. 5.5-1. Particle size distribution of dust samples D3 and D4. 
in dust samples D3 and D4 peak in the range of 2.0 to $2.6 \mu \mathrm{m}$. For comparison, the results of the sieve analysis, given in Tables 5.2-3 and 5.2-4 in terms of mass percent, are shown at the right of Fig. 5.5-1. The largest size fraction for the D3 sample was the fines (<44 $\mu \mathrm{m})$, whereas the major part of sample $D 4$ was in a size range of 44 to $150 \mu \mathrm{m}$.

\subsection{Characterization of "Soot" Collected from Exterior Surface of Fuel Elements}

Approximately $200 \mathrm{mg}$ of black, fine powder was collected from the exterior surface of driver-fuel elements EO6-OI (removed after 384 EFPDS of irradiation) and FO5-05 (extracted at end of reactor life). The appearance of these deposits may be seen in some of the photographs of the external surface of driver element Ell-07 shown in ref. 5. This powder was found as a uniform deposit adhering to the external graphite in the upper reflector region. The samples were acquired by brushing the powder off gently with a toothbrush.

Figure 5.6-1 shows an SEM micrograph of a sample of the "soot" taken from the surface of fuel element F05-05 before any attempt was made to de-agglomerate the particles. The "soot" is seen here to consist of a tangled mat of filamentary particles ranging from $\sim 0.1$ to $0.5 \mu \mathrm{m}$ in width plus some flake-like particles up to $4 \mu \mathrm{m}$ in size.

Attempts to de-agglomerate these fine particles in order to obtain an accurate particle size distribution were not successful; instead, a particle de-agglomeration was achieved which allowed a more detailed visual inspection of individual particles. Figure 5.6-2 shows two views of such a partially de-agglomerated sample of "soot" at 10,000X magnification.

The present best judgement, which is not conclusive at this time, is that this fuel element "soot" consists mainly of graphite deposited from gaseous hydrocarbons, carbon dioxide, or carbon monoxide. This view is supported by $x$-ray diffraction measurements on samples drawn from both fuel elements $\mathrm{EO}-\mathrm{O} 1$ and F05-05. In addition to characteristic graphite lines, other faint, unidentified lines were observed on the diffraction 

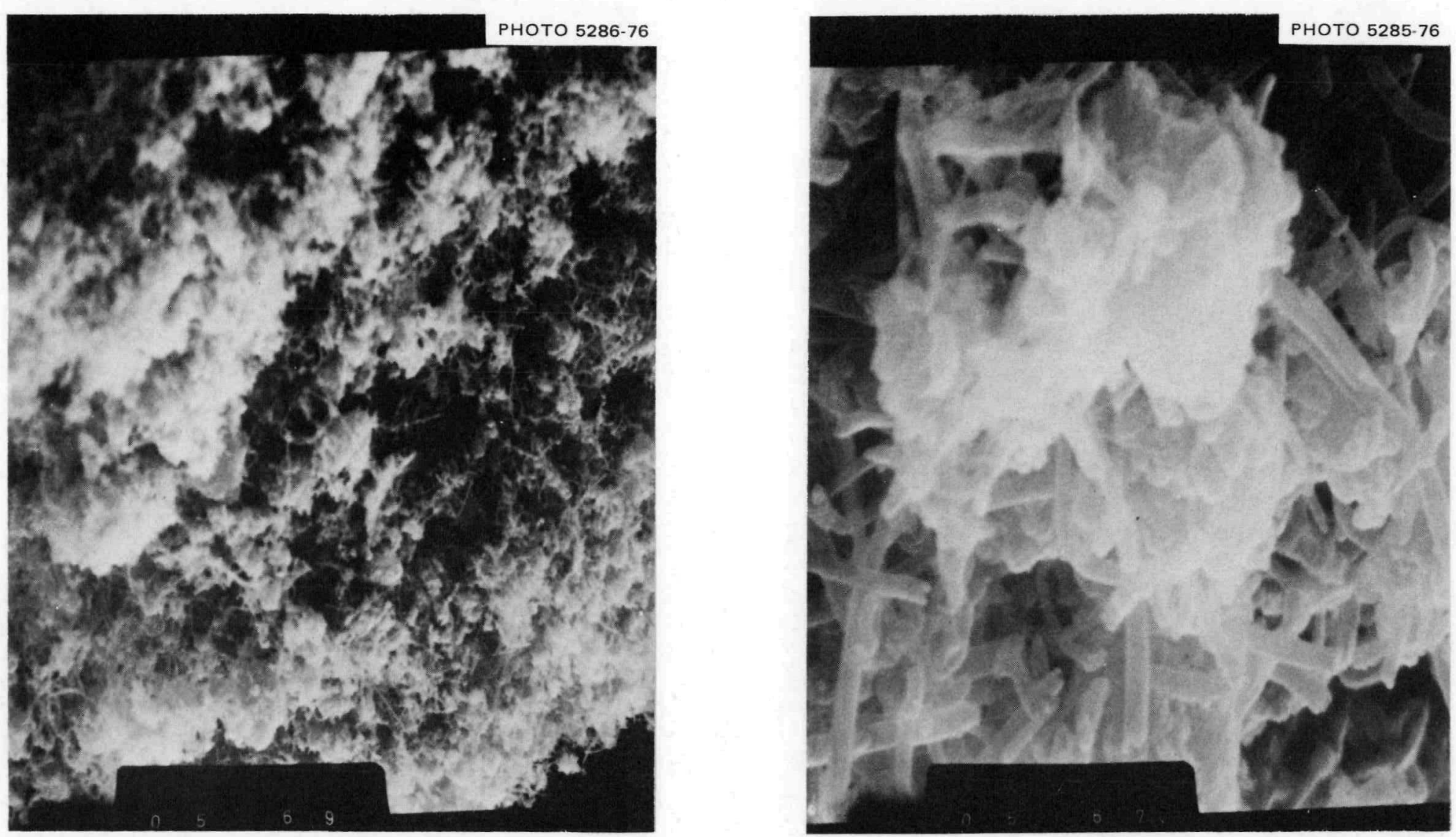

Fig. 5.6-1. SEM micrograph of "soot" collected from the outside surface of driver-fuel element F05-05; magnification $=1000 \mathrm{X}$ (left) and 10,000X (right). 

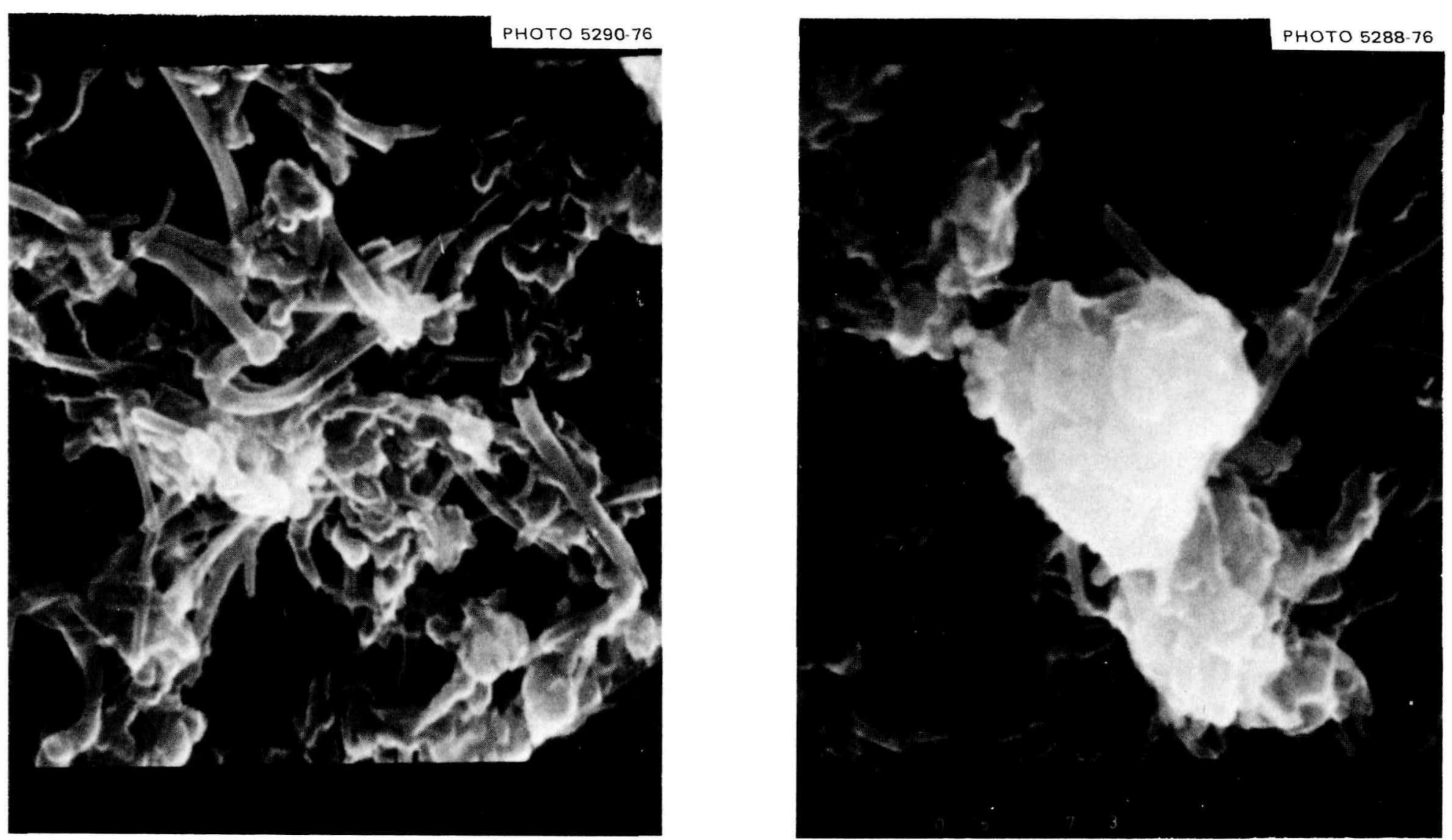

Fig. 5.6-2. Partially de-agglomerated "soot" collected from the outside surface of fuel element F05-05; magnification = 10,000X. 
pattern. Filamentary growths such as those seen here have been observed. by graphite researchers in the field of vapor deposition. 6 It is interesting to point out that these filaments appear to be hollow (note the filament endings visible at left in Fig. 5.6-2). The other, nonbroken end of filament appears to be a button of some material which may provide the active site for growth.

The layered particles observed, such as the 4-um-diam particle seen at right in Fig. 5.6-2, may originally have been an amorphous carbon deposit which crystallize under the influence of neutron irradiation.

A third view of the partially de-agglomerated "soot" is shown in Fig. 5.6-3 at 3,000 and 10,000X magnification. The filament in the central portion of the left view had a $0.5-\mu \mathrm{m}$ diameter--the largest filament to be observed. On the right, particulates as small as $0.1 \mu \mathrm{m}$ can be seen.

In the absence of a quantitative particle size analysis, it is hoped Figs. 5.6-1 to 5.6-3 adequately illustrate a representative physical description of the fuel element "soot". Initially, the main interest in this material was the belief that it resulted from deposited particulates and, as such, was representative of circulating solids in the primary coolant. It now appears that this material more likely grew in place, and, hence, instead represents one source of the circulating particulate matter.

Chemical analysis of the material collected from element F05-05 revealed the major constituent to be carbon which comprised $92 \%$ of the sample mass. Almost all of the remainder (7.94\% of the sample) was iron. Oxygen was sought but not found, indicating a presence of $<0.5 \%$. In addition, the elements $\mathrm{Al}, \mathrm{B}, \mathrm{Cd}, \mathrm{Cr}, \mathrm{Cu}, \mathrm{Mn}, \mathrm{Mo}, \mathrm{Ni}, \mathrm{Pb}, \mathrm{Pd}, \mathrm{Pt}, \mathrm{Sb}, \mathrm{Si}$, $\mathrm{Sn}$, Ti, V, and Zr were sought spectrographically but not found, signifying they were present at concentrations less than indicated in Table 5.6-1.

Examination of the $\mathrm{x}$-ray emission spectrum obtained during the SEM process would reveal constituents that are above magnesium in the periodic table. Such examination of F05-05 soot indicated iron to be the principal detectable substance, with low levels of the elements Si, Mo, Ru, and Nb also present. 

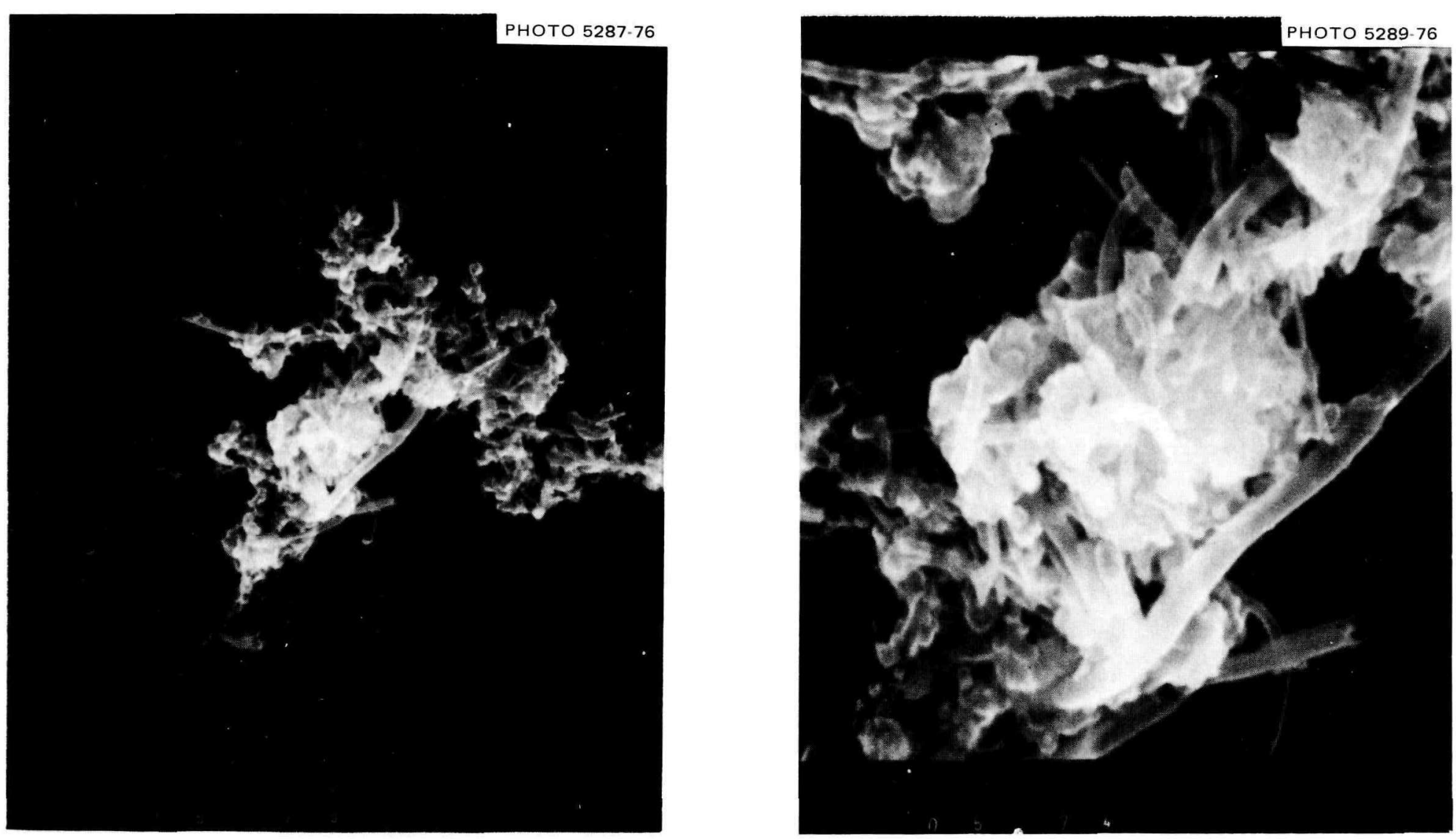

Fig. 5.6-3. Partially de-agglomerated sample of "soot" from the outside surface of fuel element F05-05; magnification = 3000X (left) and 10,000X (right). 
Table 5.6-1. Results of spectrographic analysis of soot found on element F05-05

(method not sensitive to carbon or oxygen)

\begin{tabular}{lc}
\hline & $\begin{array}{c}\text { Concentration } \\
(w t \%)\end{array}$ \\
\hline $\mathrm{AI}$ & $<0.01$ \\
$\mathrm{~B}$ & $<0.01$ \\
$\mathrm{Cd}$ & $<0.2$ \\
$\mathrm{Cr}$ & $<0.01$ \\
$\mathrm{Cu}$ & $<0.003$ \\
$\mathrm{Mn}$ & $<0.005$ \\
$\mathrm{Mo}$ & $<0.01$ \\
$\mathrm{Ni}$ & $<0.02$ \\
$\mathrm{~Pb}$ & $<0.2$ \\
$\mathrm{Pd}$ & $<0.1$ \\
$\mathrm{Pt}$ & $<0.1$ \\
$\mathrm{Sb}$ & $<0.1$ \\
$\mathrm{Si}$ & $<0.01$ \\
$\mathrm{Sn}$ & $<0.05$ \\
$\mathrm{Ti}$ & $<0.03$ \\
$\mathrm{~V}$ & $<0.01$ \\
$\mathrm{Zr}$ & $<0.01$ \\
\hline
\end{tabular}

${ }^{a}$ Silicon as $\mathrm{SiO}_{2}$ would have been lost in sample preparation.

Neutron activation analysis revealed that $1.4 \mathrm{ppm}{ }^{235} \mathrm{U}$ was present in F05-05 soot.

The major, observed gamma- and beta-emitting nuclides observed in the soot are listed in Table 5.6-2 for samples drawn from a 384-day (E06-01) and 900-day (F05-05) fuel element. The levels are given in terms of $\mu \mathrm{Ci} / \mathrm{g}$ corrected back to the termination of irradiation service. It is interesting to note that these observed levels are comparable to the maximum activity found in the sleeve and spine graphite itself ${ }^{5}$ on a per unit mass basis.

In summary, it appears that the "soot" collected from the upper reflector region of fuel element F05-05 is indeed soot. This material was evidently deposited from gaseous hydrocarbons in the coolant as 
Table 5.6-2. Gamma- and beta-emitting nuclides levels found in fuel element soot, corrected to date of fuel element removal

\begin{tabular}{|c|c|c|}
\hline & \multicolumn{2}{|c|}{ Soot deposit removed from fuel element } \\
\hline & $\begin{array}{l}\mathrm{E} 06-01^{\mathrm{a}} \\
(\mu \mathrm{Ci} / \mathrm{g})\end{array}$ & $\begin{array}{l}\text { F05-0 } 5^{b} \\
(\mu \mathrm{Ci} / \mathrm{g})\end{array}$ \\
\hline $\mathrm{Mn}-54$ & 29.4 & 32.5 \\
\hline $\mathrm{Co}-60$ & 53.1 & 84.9 \\
\hline Zr-95 & $2.2 \pm 3$ & \\
\hline $\mathrm{Nb}-95$ & $2.0 \mathrm{E} 5$ & \\
\hline $\mathrm{Ag}-11 \mathrm{Om}$ & & 34.0 \\
\hline$S b-125$ & 4.84 & \\
\hline $\mathrm{Cs}-134$ & 29.1 & $4.19 \mathrm{E} 3$ \\
\hline $\mathrm{Cs}-137$ & 87.8 & $2.38 \mathrm{E3}$ \\
\hline $\mathrm{Ce}-144$ & $1.03 \mathrm{E} 3$ & \\
\hline Eu- 154 & & 36.7 \\
\hline $\mathrm{H}-3$ & & \\
\hline Sr-89 & $1.0 \mathrm{E3}$ & \\
\hline $\operatorname{Sr}-90$ & 25.0 & \\
\hline
\end{tabular}

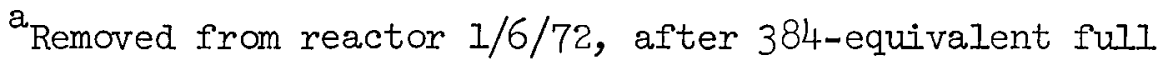
power (EFPD) service.

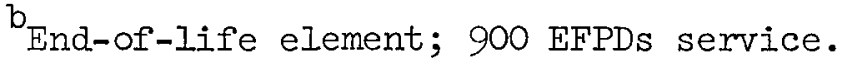

either the tubular structure or as the particulates shown in Figs. 5.6-1 to 5.6-3. These soot deposits were probably a source of particulates circulating with the primary coolant.

5.7 Dust Concentration and Production Rate in the Primary System

A mass balance of dust in the size range $\Delta d$ about d may be written:

$$
V \frac{d C_{p}(d, \Delta d)}{d t}=S_{p}(d, \Delta d)-C_{p}(d, \Delta d)\left(N(d) Q_{d c}+Q_{p}\right) \text {, }
$$

where

$$
\begin{aligned}
\mathrm{V} & =\text { primary system volume, } \mathrm{cm}^{3}, \\
\mathrm{C}_{\mathrm{p}}(\mathrm{d}, \Delta \mathrm{d}) & =\text { concentration of dust of size } \Delta \mathrm{d} \text { about } \mathrm{d}, \mathrm{g} / \mathrm{cm}^{3}, \\
\mathrm{~S}_{\mathrm{p}}(\mathrm{d}, \Delta d) & =\text { dust production rate, } \mathrm{g} / \mathrm{sec}, \\
\mathrm{N}(\mathrm{d}) & =\text { collector efficiency, }
\end{aligned}
$$




$$
\begin{aligned}
Q_{\mathrm{dcc}}, Q_{p}= & \text { flow rates to the dust collector and purification } \\
& \text { system, } \mathrm{cm}^{3} / \mathrm{sec} \text {. }
\end{aligned}
$$

Equation (1) implicitly assumes the dust concentration to be independent of location within the primary system. The collection efficiency, $\mathrm{N}$, of the cyclone dust collectors is approximately equal to that given in Fig. 5.1-2 where it is noted that particles greater than $\sim 5 \mu$ are collected with high efficiency. Since the smallest particles noted in the SEM micrographs obtained for size fractionated portions of dust samples D3 and D4 were on the order of $10 \mu \mathrm{m}$, the collection efficiency of all particles observed in the dust collections was unity, and the functional dependency on particle size may be dropped. In this case, the dust production rate at steady state may be computed from:

$$
S=C_{p}\left(Q_{d c}+Q_{p}\right)
$$

The mass flow rate through each of the two dust collectors is given to be $2475\left(1 \mathrm{~b}_{\mathrm{m}} / \mathrm{hr}\right)$, whereas the mass flow to the purification system, which also serves to remove dust from the primary system, is given as $1000\left(1 \mathrm{~b}_{\mathrm{m}} / \mathrm{hr}\right)$. This yields the volumetric flows of $1.62 \times 10^{5}$ and $6.55 \times 10^{4} \mathrm{~cm}^{3} / \mathrm{sec}$ for $Q_{d c}$ and $Q_{p}$, respectively, based on a typical cold leg density for helium. The total dust purification flow (for two dust collection systems plus the purification flow) is, therefore, equal to $3.89 \times 10^{5} \mathrm{~cm}^{3} / \mathrm{sec}$.

The dust concentration in the primary systems may be obtained from the mass of dust collected from:

$$
m_{p}(d, \Delta d)=Q_{d c} \cdot N(d) \cdot C_{p}(d, \Delta d) \cdot T_{c},
$$

where

$$
\begin{aligned}
\mathrm{mp}(\mathrm{d}, \Delta \mathrm{d}) & =\text { total mass collected in size range } \Delta \mathrm{d} \text { about } \mathrm{d}, \mathrm{g}, \\
\mathrm{T}_{\mathrm{c}} & =\text { collection time, sec. }
\end{aligned}
$$

As previously shown, since no particles less than $10 \mu$ in size were observed, collection efficiencies are unity for all the entire size range, and the dependency of particle size may be dropped in Eq. (3) also. 
Calculated results for the loop dust concentration during the operating periods when samples D2, D3, and D4 were collected, as well as the estimated dust production rates for these periods, are summarized in Table 5.7-1. Dust concentrations were obtained using Eq. (3) and employing the value of the dust separator flow for one loop. The dust production rate was computed using Eq. (2) where the dust collector volume flow rate for both collection loops is appropriate.

An interesting feature of the results shown in Table 5.7-1 is that the primary loop dust level and dust production rate evidently dropped continuously during the course of core 2 operation. The total observed drop was a factor of $\sim 5$ between the final 10-month operating period relative to that for the first nine months of Core 2 operation.

\section{References for Section 5}

1. Peach Bottom Atomic Power Station Final Hazards Summary Report, Part C, Fig. II-91, Philadelphia Electric Co. (Mar. 3, 1964).

2. Peach Bottom Atomic Power Station Final Hazards Summary Report, Part C, Fig. II-191, Philadelphia Electric Co. (Mar. 3, 1964).

3. C. E. Lapple, Fluid and Particle Mechanics, Univ. of Del. (1954), Chap. 14.

4. Peach Bottom 4tomic Power Station Final Hazards Summary Report, Part C, Philadelphia Electric Co., Docket-50171-2 (Mar. 3, 1954).

5. R. P. Wichner et al., Distribution of Fission Products in Peach Bottom HTGR Fuel Element El1-07, ORNL-5214 (Oct. 1976́), Sect. 4.

6. W. H. Eatherly, Metals and Ceramics Division, ORNI, personal comunication, Aug. 1976. 
Table 5.7-1. Primary system dust concentrations and production rates estimated from cyclone dust collector samples

\begin{tabular}{|c|c|c|c|c|c|}
\hline $\begin{array}{c}\text { Dust } \\
\text { sample }\end{array}$ & Operating period & $\begin{array}{l}\text { Sample mass, } \\
\text { loop } 1 \\
\text { (g) }\end{array}$ & $\begin{array}{l}\text { Dust c } \\
\text { in prima: } \\
\text { opera } \\
\text { (pg/scc } \\
\text { coolant) }\end{array}$ & $\begin{array}{l}\text { tration } \\
\text { op during } \\
\text { period } \\
\text { (mg/kg } \\
\text { coolant) }\end{array}$ & $\begin{array}{l}\text { Rate of dust } \\
\text { production during } \\
\text { operating period } \\
\qquad(g-s e c)\end{array}$ \\
\hline D2 & July 15, 1970-Apr. 24, 1971 & 80.0 & 21.0 & 0.12 & $8.2 \times 10^{-6}$ \\
\hline D3 & June 18, 1971-Sept. 14, 1973 & 110.0 & 15.0 & 0.084 & $5.8 \times 10^{-6}$ \\
\hline D4 & Jan. 5, 1973-Oct. 31, 1974 & 15.3 & $4 \cdot 3$ & 0.024 & $1.7 \times 10^{-6}$ \\
\hline
\end{tabular}


APPENDIX A: DOCUMENTATION OF GAMMA SPECTROSCOPY METHODS

The validity of the gamma spectroscopy results reported in this study rests on the accuracy of the reference nuclear data (half-lives, etc.) used in calculations, methods of photpeak measurement, and procedures for calibration and use of counting efficiencies. A documentation of these factors as they were applied in this study is presented below.

\section{Al. Reference Nuclear Data Source}

The values of half-lives, gamma-ray energies, and branching ratios (gamma quanta of each energy emitted per disintegration) for the radionuclides measured were taken from a tabulation by Dyer and Bate. ${ }^{I}$

\section{A2. Measurement of Photopeak Count Rates and Energies}

\section{A2.I Peak finding and single peak measurement}

All gamma-ray spectra were resolved by computer programs that found photopeaks and measured their net count rates and energies in the following manner. A search was made to locate photopeaks by a comparison of a statistical function, $\mathrm{XF}$,

$$
X F=\frac{C_{i+1}-C_{i-1}}{\sqrt{C_{i}}},
$$

with a test quantity $T$. Symbol $C$ denotes the counts in a channel, and $i \pm 1$ denotes channels adjacent to channel $i$. The value of $T$ was normally set equal to 3 . In flat "compton regions" of spectra, the value of XF has a nominal value of $\sim \pm 1$.

On the low energy side of a photopeak, XF becomes much larger than 3 (reaching several hundred for large peaks) and becomes much smaller than -3 on the high energy side. Thus, a peak is found by the detection of the rapidly changing counts per channel on its sides. The channel centroid of the peak, PC, was computed by: 


$$
\mathrm{PC}=\frac{\sum_{i=\mathrm{X}_{0}-1}^{\mathrm{X}_{0}+1} \mathrm{C}_{i} \cdot \mathrm{x}_{i}}{\sum_{i=\mathrm{X}_{0}-1}^{\mathrm{X}_{0}+1} \mathrm{C}_{i}}
$$

where

$X_{0}=$ peak channel containing the largest number of counts,

$X_{i}=$ the ith channel number.

The peak energy, E, was then calculated from:

$$
E=E_{0}+D(P C) \text {, }
$$

where $\mathrm{E}_{0}$ is the energy intercept or energy corresponding to channel $\mathrm{O}$, and $D$ is the energy slope in $\mathrm{keV} /$ channel.

The net counts in the peaks were then determined as follows. Beginning with the first peak, a search was made from its centroid toward its low energy side until two consecutive values of $A B S(X F)^{*}$ were less than $T$. The search was then made toward the peak's high energy side. In this manner, four baseline channels having statistically equivalent counts were located on each side of the peak. This method of peak detection and area measurement (counts in the peak) is illustrated in Fig. A.1. The peak region over which the count rate was determined is shown to lie between the second channels, $X_{1}$ and $X_{2}$, of the quartets of equal count channels on both sides of the peak. The number of counts, BL, under the linear baseline was computed by:

$$
\mathrm{BI}=1 / 4\left(\mathrm{X}_{2}-\mathrm{X}_{1}+1\right)\left(\mathrm{C}_{\mathrm{X}_{1}}+\mathrm{C}_{\mathrm{X}_{1}+1}+\mathrm{C}_{\mathrm{X}_{2}}+\mathrm{C}_{\mathrm{X}_{2}-1}\right)
$$

The net count, NC, in the peak was calculated by:

$$
\mathrm{NC}=\sum_{i=\mathrm{X}_{1}}^{\mathrm{X}_{2}} \mathrm{C}_{i}-\mathrm{BL}
$$

*ABS (XF) denotes absolute value of XF. 
ORNL DWG $76-955$

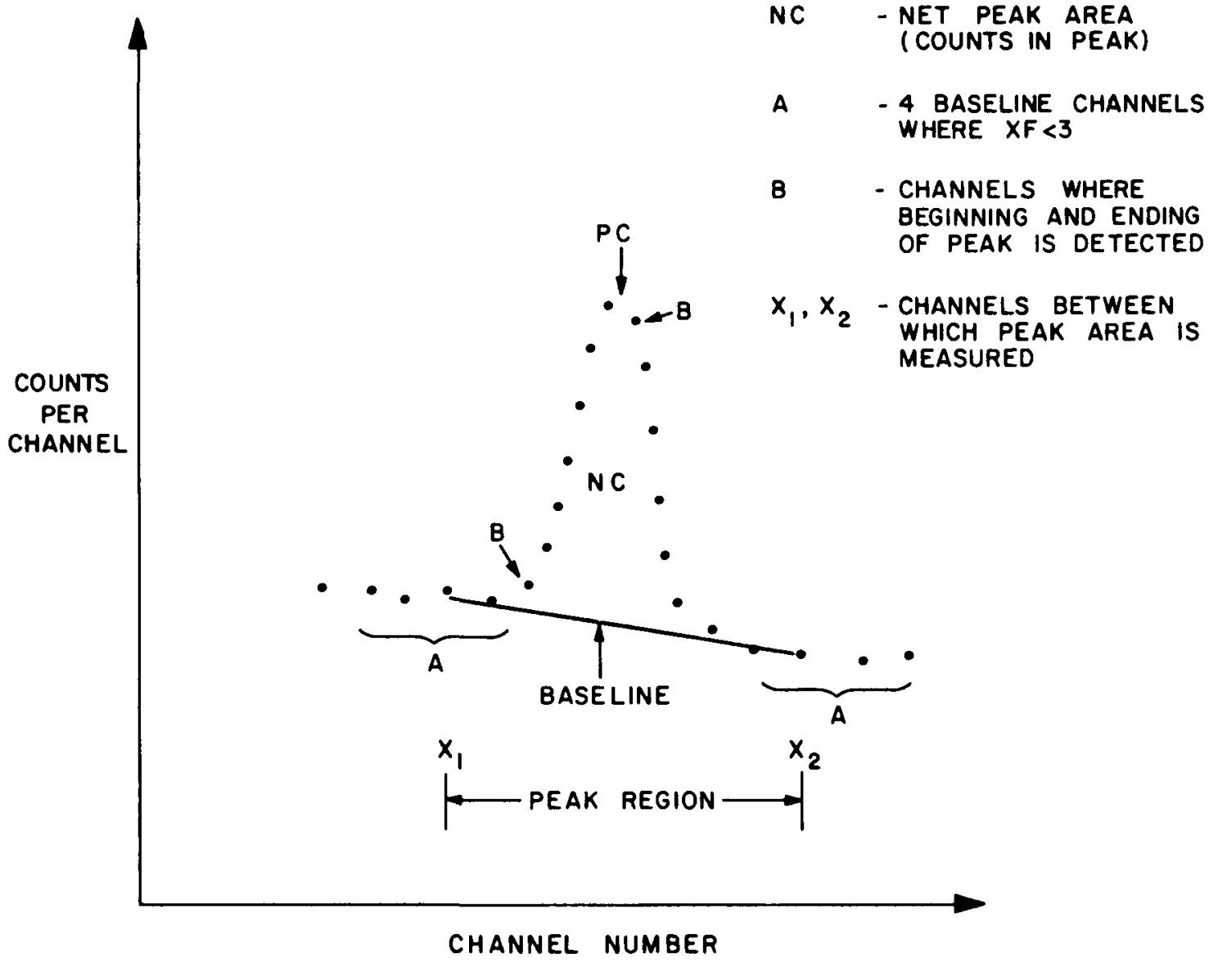

Fig. A.I. Illustration depicting the detection and measurement of single photopeak. 
The net count rate, $R$, of a photopeak per unit weight of sample was computed by:

$$
R=\frac{\mathrm{NC} \cdot \exp \left(\mathrm{K} \cdot \mathrm{R}_{\mathrm{T}}\right)}{\mathrm{W} \cdot \mathrm{T}_{\mathrm{L}}},
$$

where

$$
\begin{aligned}
\mathrm{W} & =\text { sample weight, } \mathrm{g}, \\
\mathrm{T}_{\mathrm{L}} & =\text { counting time in live, sec, } \\
\mathrm{R}_{\mathrm{T}} & =\text { count rate in the entire spectrum, } \\
\mathrm{K} & =\text { an empirically measured constant having a value between } \\
& \sim 3 \times 10^{-6} \text { and } 6 \times 10^{-6} \mathrm{sec} .
\end{aligned}
$$

The factor $\exp \left(K \cdot R_{T}\right)$ corrects for pulse pileup in the amplifier, which results in counts being lost from photopeaks. The extent of the loss normally amounts to $\sim 1$ to $3 \%$ per 1000 counts $/ \mathrm{sec}$ for $R_{\mathrm{T}}$. The value of $\mathrm{K}$ was determined by measuring a series of ${ }^{137} \mathrm{Cs}$ standards whose relative activities were accurately known. The exact value of $K$ depends on the settings chosen for the integration and differentiation times of the amplifier.

\section{A2.2 Analysis of multiplet peak regions}

In those instances in which photopeaks lie close to each other, for instance, the 795 - and $801-\mathrm{keV}$ peaks of ${ }^{134} \mathrm{Cs}$, the detection of the upper-energy baseline quartet of channels may not be found until two or more photopeaks are included in the peak region. This situation is illustrated for a doublet in Fig. A.2. The determination of the count rates of the individual peaks was done as follows. The total number of net counts in the region was computed as before. A linear relation expressing the counts per channel, $B_{i}$, in the baseline was derived by the equation:

$$
B_{i}=I+S \cdot X_{i},
$$

where

$$
\begin{aligned}
& I=\text { the intercept, } \\
& S=\text { the slope of the line. }
\end{aligned}
$$




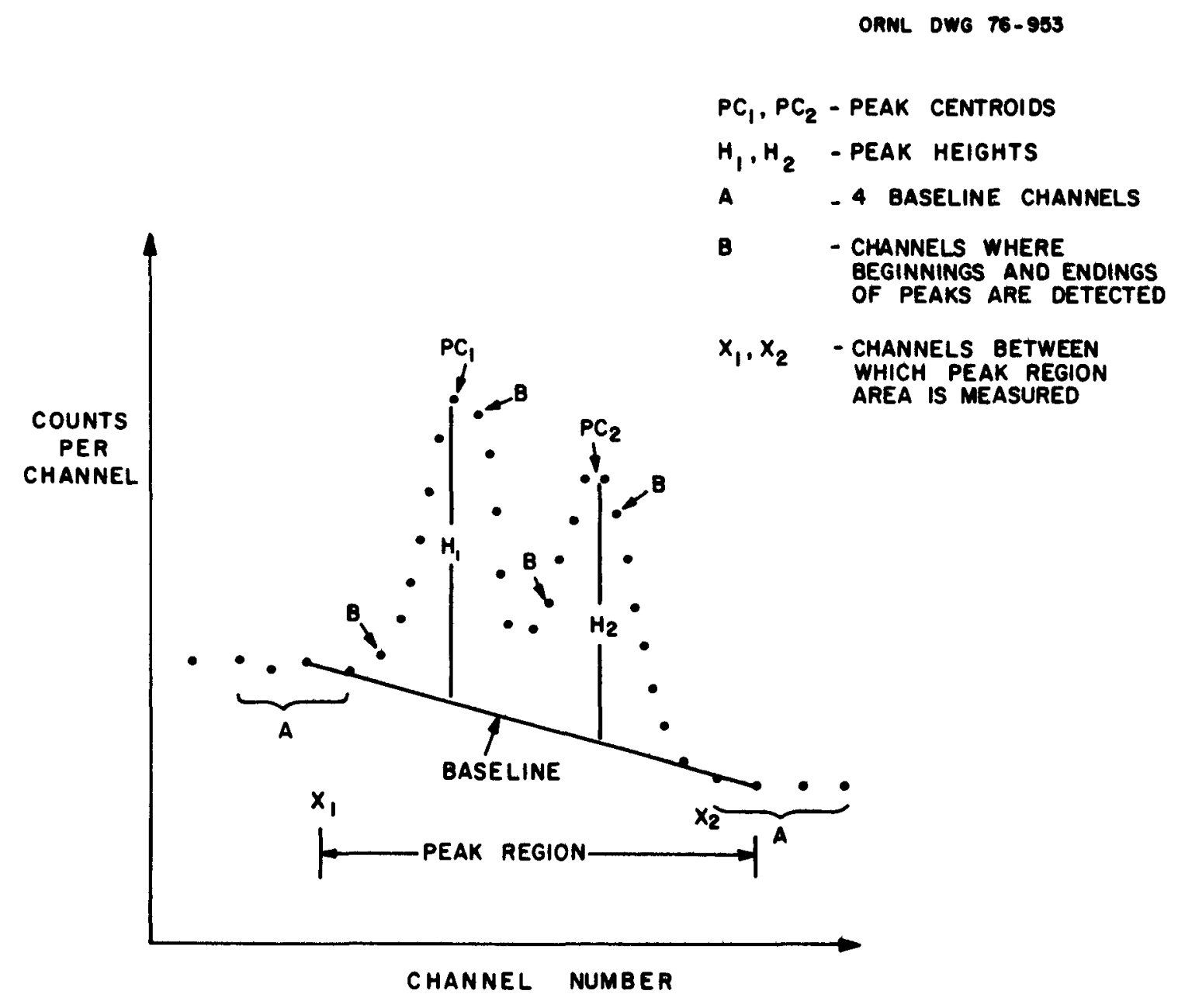

Fig. A.2. Illustration depicting the detection and measurement of photopeak doublet. 
The photopeak heights, $\mathrm{H}_{i}$, were computed from the equation:

$$
\mathrm{H}_{i}=\mathrm{C}_{\mathrm{X}_{0}}-\mathrm{B}_{0}
$$

where $\mathrm{x}_{0}$ denotes the channel having the maximum number of counts. The net count rates of the photopeaks were then computed from the total net counts in the region and the peak heights by the relation:

$$
R_{i}=\frac{N C \cdot H_{i} \exp \left(K \cdot R_{T}\right)}{W \cdot T_{L} \sum_{i=1}^{\mathbb{N}} H_{i}},
$$

where

$$
\begin{aligned}
& i=\text { the peak number, } \\
& N=\text { the number of peaks in the region. }
\end{aligned}
$$

This method of proportioning peak count rates according to their heights was tested and found to yield values of acceptable accuracy for the limited number of peak multiplets encountered in this study.

\section{A3. Counting Efficiencies}

\section{A3.1 Usage of counting efficiencies}

The counting efficiency--counts recorded in a photopeak per photon emitted from a radioactive sample--is a function of gamma energy and geometrical factors. In this work, the counting efficiencies, EF, at several gamma energies were empirically determined, and the resulting values fit by linear least squares to the relation:

$$
\text { In }(\mathrm{EF})=\mathrm{C}_{1}+\mathrm{C}_{2} \ln \mathrm{E}+\mathrm{C}_{3}(\operatorname{In} \mathrm{E})^{2}+\mathrm{C}_{4}(\operatorname{In} \mathrm{E})^{4} \text {, }
$$

where $E$ denotes the gamma energy in $\mathrm{keV}$. Counting efficiencies were then computed from this expression as needed during resolution of a gamma spectrum. It is common practice to represent the counting efficiency by one set of coefficients for energies below $\sim 200 \mathrm{keV}$ and by another set for energies above $200 \mathrm{keV}$. This practice was followed. Above $200 \mathrm{keV}$, a nearly linear relation normally exists between In (EF) and In (E) so that two coefficients suffice. In the case of the measurements in the 
Peach Bottom coolant ducts, the extreme gamma-ray attenuation of the duct wall caused the relation to be nonlinear. Except for the fission gases and the $133-\mathrm{keV}$ gamma ray of ${ }^{144} \mathrm{Ce}$, all gamma rays measured in this work had energies above $300 \mathrm{keV}$. Thus, in most instances, one polynominal with two coefficients was used to describe the counting efficiency.

\section{A3.2 Experimental determination of counting efficiencies}

Many of the measurements in this study consisted of acquiring gamma spectra of samples that approximated point sources with minimal corrections for gamma-ray attenuation in the sample and its container. Such samples included diffusion tube segments, dust samples, coolant sampler filters, etc. Counting efficiencies for these samples were determined from measurements on several primary standard sources purchased from the Intemational Atomic Energy Agency, the National Bureau of Standards, and Amersham Radiochemical Center. The use of these primary standards was previously discussed. ${ }^{2}$ out of all of the primary standards used, no disagreement was found that appeared to be larger than $\sim \pm 3 \%$. Thus, it is believed that errors caused by uncertainties in counting efficiencies for measurments on small samples were generally less then $\sim \pm 5 \%$. In some cases (e.g., ${ }^{13}{ }^{I} I$ on diffusion tube segments), the very 1 ow Ievels of radionuclides in samples resulted in counting statistic errors considerably larger than $\pm 5 \%$.

Samples that did not resemble a point source included the coolant ducts on which plateout activities were measured, entire gaseous diffusion tubes, and fission-gas sources consisting of the hot-valve cooling line, vials in which purge gas was counted, and the flow cells used to measure primary circuit coolant. Except for the hot-valve cooling line, it was possible to relate all measurements back to the primary standards through the use of secondary standards calibrated against the primary standards. The procedures used to establish counting efficiencies of these unusual sources are discussed below. 


\section{A3.2.1 Hot-valve cooling line}

Manganese-56, which has gamma rays with energies of 846 , 1811, and $2112 \mathrm{keV}$, was found in this pipe. Its intensity was relatively high, and its photopeaks were well resolved. The counting efficiency for a gamma ray of ${ }^{56} \mathrm{Mn}$ in the pipe can be written as:

$$
\mathrm{EF}=\frac{\mathrm{R}}{\mathrm{S}(\mathrm{BR})} \text {, }
$$

where $R$ is the net photopeak count rate, $S$ is the source strength in disintegrations per second per $\mathrm{cm}^{2}$ of pipe surface or per $\mathrm{cm}^{3}$ of coolant, and $B R$ is the branching ratio of the gamma ray. Since $S$ is a constant for all the gamma rays of ${ }^{56} \mathrm{Mn}$, we can write:

$$
E F \cdot S=R_{f}=\frac{R}{B R} \text {, }
$$

where $R_{f}$ is termed the relative counting efficiency. Since the relative counting efficiency and the absolute counting efficiency have the same shape curve when plotted against energy, it was possible to normalize the relative counting efficiency curve obtained from the ${ }^{56} \mathrm{Mn}$ to an absolute counting efficiency curve. This normalization was accomplished from the count rate data obtained from a well-resolved photopeak of ${ }^{88} \mathrm{Kr}$ and the source strength of ${ }^{88} \mathrm{Kr}$ measured in the coolant by Philadelphia Electric Company (see sect. 4).

\section{A3.2.2 Primary circuit piping}

Counting efficiencies--the ratio of net photopeak count rates to photon emission rates per $\mathrm{cm}^{2}$ of duct surface--were determined on a mock-up that simulated the radioactive duct. These calibration measurements were carried out after each experiment at Peach Bottom, that is, in 1971, 1972, 1973, and 1974. Except for the 1974 measurements, the mock-up consisted of a section of blotter paper to which was applied a uniform coating of ${ }^{140}$ La. The coating was painted on the paper by the use of a paint in which ${ }^{140}$ La was uniformly dispersed. The paper was rolled into a cylinder with a diameter equal to the Peach Bottom duct and a length of $\sim 0.6 \mathrm{~m}$ (2 ft). In 1974, the mock-up consisted of a coated paper cylinder 
placed snugly inside a steel cylinder with a wall thickness of $0.95 \mathrm{~cm}$ (3/8 in.). This thickness was $0.16 \mathrm{~cm}$ (1/16 in.) less than the Peach Bottom duct. The thermal insulation that surrounded the reactor duct was not used on the outside of any of the mock-ups. Gamma spectra of each mock-up were acquired for each detector-duct counting configuration used at Peach Bottom nominally at three locations around the mock-up separated by $90^{\circ}$.

Counting efficiencies, EF, for the Peach Bottom duct were determined at energies corresponding to each of the major gamma rays of ${ }^{140} \mathrm{La}$ from the expression:

$$
E F=\frac{R F}{S(B R) 3.7 E 4},
$$

where

$R=$ the net photopeak count rate measured with a mock-up, $F=$ a factor that corrects for the difference in gamma-ray attenuation of the reactor duct and the mock-up and is equal to the fraction of gamma rays transmitted through material in the duct wall that is not present in the mock-up wall, $\mathrm{S}=$ the specific surface activity, $\mu \mathrm{Ci} / \mathrm{cm}^{2}$, of ${ }^{140} \mathrm{La}$ on the mock-up,

$\mathrm{BR}=$ the branching ratio, photons emitted per disintegration of $140 \mathrm{La}$,

$3.7 \mathrm{E} 4=$ the conversion factor, disintegrations/sec $\mu \mathrm{Ci}$.

These efficiencies were than used as described in sect. A3.1.

Since measurements before 1974 were made with a mock-up that consisted only of a paper cylinder, the factor $F$ included the effects of gamma-ray attenuation in the total duct wall--1.11 cm (7/16 in.) of carbon steel plus the thermal insulation surrounding the duct. The 1974 mock-up consisted of a steel cylinder with a $0.95-\mathrm{cm}$ (3/8-in.) wall thickness, and the factor $F$ included only the attenuation for an additional $0.16 \mathrm{~cm}$ ( 1.16 in.) of steel and the thermal insulation. It should be noted that the detector used in 1974 was not the one used previously, and the 
measurements made on the 1974 mock-up were not applicable to prior calibrations. The attenuation of gamma rays in carbon steel and the thermal insulation was measured separately, and values of $F$ for the insulation and the appropriate thickness of steel were derived and multiplied to yield a value of $F$ in Eq. (6). Attenuations were derived from gamma spectra of ${ }^{226} \mathrm{Ra}$ acquired with and without sections of the insulation and the appropriate thicknesses of steel interposed between the radium source and a $\mathrm{Ge}(\mathrm{Li})$ detector. A typical specimen of the insulation was removed from the duct for this measurement following final reactor shutdown. Initially, an atypical specimen of insulation used for this determination resulted in an estimate of the duct activities that was $\sim 40 \%$ too large (see refs. 3 and 4 , sect. 2).

Following the acquisition of gamma spectra on each mock-up, three specimen of known area were cut from the paper cyclinder and counted to determine the specific surface activity of ${ }^{140} \mathrm{La}$. These measurements, in addition to providing a value $S$ in $E q$. (6), also served to show the adequacy of the coating uniformity of ${ }^{140} \mathrm{La}$ on the paper.

\section{A3.2.3 Gaseous diffusion tubes}

To determine the inventories of radionuclides in total diffusion tubes, the tubes were wrapped in a spiral and placed snugly around the end cap of a Ge(Li) detector. Counting efficiencies for this counting geometry were derived from a standard tube counted in the same manner. A piece of thin iron wire $54 \mathrm{~cm}$ in length (length of the diffusion tube) was neutron activated to produce ${ }^{56} \mathrm{Mn}$ and ${ }^{59} \mathrm{Fe}$. The wire was threaded into a blank diffusion tube which was wrapped in a spiral and counted. The wire was then removed from the tube, wrapped into a small ball, and counted at a geometry that had been standardized with the primary standards. The resulting data were used to obtain a plot of the counting efficiency vs gamma energy for the tube wrapped around the detector.

\section{A3.2.4 Purge gas vials and flow cells}

Counting efficiencies for the 2.7-cc vials in which purge gas samples were analyzed were derived as follows. A sample of fission gas was obtained 
in one of these vials with sufficient ${ }^{88} \mathrm{Kr}$ to permit measurement at a standard counting geometry. After most of the decay of the shorterlived gases had occurred, the vial was measured, and the activity of 88

$\mathrm{Kr}$ was determined. The ${ }^{88} \mathrm{Kr}$ was then allowed to decay for several hours, and the vial was again counted at the same counting geometry used for the purge gas samples. The resulting data was used to compute counting efficiencies for this geometry for all of the major gamma rays of $88 \mathrm{Kr}$.

Counting efficiencies for the flow cells were derived in a similar manner. A sample of fission gases was obtained in a small vial, and its $88 \mathrm{Kr}$ content was measured at a standara counting geometry. The gas was quantitatively transferred to a flow cell which was counted on the detector end cap. These data were used to derive counting efficiencies for the flow cells.

A4. Formula Used to Compute Activities and Fission

Gas Concentrations

The activity, $\mathrm{A}(\mu \mathrm{Ci} / \mathrm{g}$ or $\mu \mathrm{Ci} / \mathrm{cc})$, of long-lived radionuclides in conventional samples was computed by the relation:

$$
A=\frac{R}{(E F)(B R) 3.7 E 4} \text {, }
$$

where

$$
\begin{aligned}
\mathrm{R}= & \text { count rate (counts per unit line second) per unit sample } \\
& \text { weight or volume, as described in sect. A.2.I, } \\
\mathrm{BR}= & \text { the branching ratio of the gamma ray, } \\
3.7 \mathrm{E} 4= & \text { the number of disintegrations per } \mu \mathrm{Ci} .
\end{aligned}
$$

In the case of those fission gas samples measured in vials (purge gas) and charcoal traps (primary coolant), it was necessary to make a correction for the decay of the short-lived gases that occurred during the counting period. The activity for these samples was computed by:

$$
A=\frac{R}{(E F)(B R) 3.7 E 4} \cdot \frac{\lambda T_{L}}{1-\exp \left(-\lambda T_{L}\right)}
$$


where

$$
\begin{aligned}
T_{L}= & \text { the live interval of counting in seconds, } \\
1-\exp \left(-\lambda T_{L}\right)= & \text { the necessary correction factor for decay during } \\
& \text { counting. }
\end{aligned}
$$

The concentration, $A_{g}$, of a fission gas in the purge gas in $\mu \mathrm{Ci} / \mathrm{cc}$ at STP (14.7 psia and $70^{\circ} \mathrm{F}$ ) within the reactor's core purge outlet was computed by:

$$
A_{g}=\frac{R}{(E F)(B R) 3.7 \mathrm{E} 4} \cdot \frac{\lambda T_{L}}{1-\exp \left(-\lambda T_{L}\right)} \cdot e^{\lambda\left(T_{d}+T_{t}\right)} \cdot \frac{P}{14.7} \text {, }
$$

where $P$ is the coolant pressure in the vial. The factor $e^{\lambda\left(T_{d}+T_{t}\right)}$ corrects for decay for the time interval, $T_{d}$, between the time the sample was taken and the time counting was begun, and for the time interval, $T_{t}$, necessary for the coolant to flow to the gas sampling station. Since the gas was collected at $\sim 70^{\circ} \mathrm{F}$, no correction for temperature was made.

In the case of the charcoal trap samples, the activity on the cell at the beginning of the spectrum acquisition was computed by Eq. (7), and the concentration of a fission gas in the coolant within the primary circuit was derived from the expression:

$$
A_{g}=A_{c} \cdot \frac{\lambda A_{c} \exp \left[\lambda\left(T_{d}+T_{t}\right)\right]}{F\left[1-\exp \left(-\lambda T_{f}\right)\right]}
$$

where

$$
\begin{aligned}
A_{c}= & \text { the number of microcuries in the cell, } \\
F= & \text { the coolant flow rate to the trap in scc per second, } \\
\mathrm{T}_{\mathrm{f}}= & \text { the time interval that the coolant was allowed to flow } \\
& \text { into the cell. }
\end{aligned}
$$

The factor $\lambda / F \cdot\left[1-\exp \left(-\lambda T_{f}\right)\right]$ corrects for decay of the fission gases during the time interval, $\mathrm{T}_{f^{*}}$.

Fission gases in the flow cell were measured at steady state, that is, no net change in their concentrations occurred during measurement. The rate of entry of a fission gas into the cell can thus be equated to the rate of disappearance from the cell, viz, 


$$
F N_{g} e^{-\lambda T_{t}}=\lambda N_{c} V+F N_{c}
$$

where

$$
\begin{aligned}
\mathrm{N}_{\mathrm{g}} \text { and } \mathrm{N}_{\mathrm{c}}= & \text { the number of atoms of the fission gas per scc } \\
& \text { within the primary circuit and in the flow cell, } \\
& \text { respectively, } \\
\mathrm{V}= & \text { the volume of gas within the cell expressed as } \\
& \text { scc. }
\end{aligned}
$$

Since the total activity of the fission gas in the cell, $A_{c}$, is equal to $\lambda \mathrm{N}_{c} \mathrm{~V}$, and the activity, $\mathrm{A}_{g}$, of the gas in the primary circuit is $(\mu \mathrm{Ci} / \mathrm{scc})$ given by:

$$
\mathrm{A}_{\mathrm{g}}=\mathrm{N}_{\mathrm{g}} \cdot \lambda \cdot 3 \cdot 7 \mathrm{E} 4,
$$

we can rewrite Eq. (10) as:

$$
A_{g}=\frac{A_{c} \lambda}{F}\left(1+\frac{F}{\lambda V}\right) \exp \left(\lambda T_{t}\right) \text {. }
$$

Expressing the volume of coolant in the cell at STP as:

$$
\mathrm{V}=\frac{\mathrm{V}_{\mathrm{c}} \mathrm{P}}{14.7}
$$

where

$$
\begin{aligned}
& \mathrm{V}_{\mathrm{c}}=\text { the physical volume of the cell }(2225 \mathrm{cc}) \\
& \mathrm{P}=\text { the pressure in the cell, }
\end{aligned}
$$

we can write:

$$
A_{g}=\frac{A_{c} \lambda}{F}\left(1+\frac{14.7 F}{\lambda V_{c} P}\right) \exp \left(\lambda T_{t}\right) .
$$

This equation was used to compute the concentrations of fission gases in the coolant within the primary circuit.

\section{References for Appendix A}

I. F. F. Dyer and L. C. Bate, "Radionuclide Decay Data," ORNL Radiation Shielding Information Center Data Library Collection, DIC-19 (1975).

2. F. F. Dyer et al., Postirradiation Examination of Peach Bottom HTGR Driver Fuel Element EO6-O1, ORNL-5126 (April 1976). 
0

0 
ORNL-5188

Dist. Category UC-77

INTERNAL DISTRIBUTION

1. J. L. Anderson

2. S. J. Ball

3. D. E. Bartine

4. L. C. Bate

5. C. D. Baumann

6. R. L. Beatty

7. J. P. Callahan

8. D. O. Campbell

9. D. A. Canonico

10. C. E. Clifford

11. J. A. Conlin

12. J. H. Coobs

13. D. A. Costanzo

14. F. L. Culler, Jr.

15. F. C. Davis

16. J. R. Distefano

17. B. C. Duggins

18-27. F. F. Dyer

28. W. P. Eatherly

29. J. F. Emery

30. D. E. Ferguson

31. Uri Gat

32. J. H. Goode

33. A. G. Grindell

34. W. S. Groenier

35. P. A. Haas

36. W. R. Hamel

37. F. E. Harrington

38. c. C. Haws

39. L. C. Hensley

40. R. F. Hibbs

4I. R. M. Hill, Jr.

42. F. J. Homan

43. J. R. Horton

44. I. D. Hulett

45. J. D. Jenkins

46. D. R. Johnson

47-56. P. R. Kasten

57. W. J. Lackey

58. K. H. Lin

59. T. B. Lindemer

60. E. L. Long, Jr.

61. R. A. Lorenz
62. A. L. Iotts

63. W. S. Lyon

64. R. E. MacPherson

65. J. C. Mailen

66. A. P. Malinauskas

67. W. R. Martin

68. W. J. Martin

69. W. J. McDowell

70. K. J. Notz

71. L. C. Oaks

72. A. R. Olsen

73. G. W. Parker

74. W. H. Pechin

75. H. Postma

76. M. F. Pruitt

77. R. H. Rainey

78. D. P. Reid

79. A. D. Ryon

80. J. P. Sanders

81. Dunlap Scott

82. J. D. Sease

83. J. H. Shaffer

84. R. L. Shepard

85. W. D. Shults

86. J. W. Snider

87. P. R. Stein

88. D. P. Stevens

89. R. S. Stone

90. M. L. Tobias

91. D. B. Trauger

92. J. E. Van Cleve

93. V. C. A. Vaughen

94-103. R. P. Wichner

104. R. G. Wymer

105-106. Central Research Library

107. Document Reference Section

108. Laboratory Records, ORNL-RC

109-118. Laboratory Records

119. ORNL Patent Office

120. E. L. Gaden, Jr. (consultant)

121. C. H. Ice (consultant)

122. R. B. Richards (consultant) 
EXTERNAL DISTRIBUTION

123. Research and Technical Support Division, ERDA-ORO, P. O. Box E, Oak Ridge, Tn. 37830

124. Director, Reactor Division, ERDA-ORO, P. O. BOx E, Oak Ridge, Tn. 37830

125-126. Director, Division of Nuclear Fuel Cycle and Production, ERDA, Washington, D.C. 20545

127-128. Director, Division of Reactor Research and Development, ERDA, Washington, D.C. 20545

129-305. Given distribution as shown in TID-4500 under category UC-77 Gas-Cooled Reactor Technology 